\title{
ASPHALT CONCRETE CHARACTERIZATION USING THE COMPLEX MODULUS TECHNIQUE
}

\author{
By \\ Yassin Eisa Adam \\ A thesis submitted to Faculty of Graduate Studies and Research \\ Under the supervision of Professor El Hussein Hassan Mohamed \\ Department of Civil and Environmental Engineering \\ Carleton University \\ Ottawa, Ontario
}

Presented to Ottawa-Carleton Institute for Civil Engineering in partial fulfilment of the requirements for the degree of Master of Applied Science in civil engineering

\author{
(C) Copyright
}

January 2005, Yassin E. Adam 


$\begin{array}{ll}\begin{array}{l}\text { Library and } \\ \text { Archives Canada }\end{array} & \begin{array}{l}\text { Bibliothèque et } \\ \text { Archives Canada }\end{array} \\ \begin{array}{l}\text { Published Heritage } \\ \text { Branch }\end{array} & \begin{array}{l}\text { Direction du } \\ \text { Patrimoine de l'édition }\end{array} \\ \begin{array}{l}\text { 395 Wellington Street } \\ \text { Ottawa ON K1A ON4 } \\ \text { Canada }\end{array} & \begin{array}{l}\text { 395, rue Wellington } \\ \text { Ottawa ON K1A ON4 } \\ \text { Canada }\end{array}\end{array}$

Your file Votre référence ISBN: 0-494-00733-8

Our file Notre référence

ISBN: 0-494-00733-8

NOTICE:

The author has granted a nonexclusive license allowing Library and Archives Canada to reproduce, publish, archive, preserve, conserve, communicate to the public by telecommunication or on the Internet, loan, distribute and sell theses worldwide, for commercial or noncommercial purposes, in microform, paper, electronic and/or any other formats.

The author retains copyright ownership and moral rights in this thesis. Neither the thesis nor substantial extracts from it may be printed or otherwise reproduced without the author's permission.
AVIS:

L'auteur a accordé une licence non exclusive permettant à la Bibliothèque et Archives Canada de reproduire, publier, archiver, sauvegarder, conserver, transmettre au public par télécommunication ou par l'Internet, prêter, distribuer et vendre des thèses partout dans le monde, à des fins commerciales ou autres, sur support microforme, papier, électronique et/ou autres formats.

L'auteur conserve la propriété du droit d'auteur et des droits moraux qui protège cette thèse. $\mathrm{Ni}$ la thèse ni des extraits substantiels de celle-ci ne doivent être imprimés ou autrement reproduits sans son autorisation.
In compliance with the Canadian

Privacy Act some supporting forms may have been removed from this thesis.

While these forms may be included in the document page count, their removal does not represent any loss of content from the thesis.
Conformément à la loi canadienne sur la protection de la vie privée, quelques formulaires secondaires ont été enlevés de cette thèse.

Bien que ces formulaires aient inclus dans la pagination, il n'y aura aucun contenu manquant. 


\begin{abstract}
Asphalt concrete (AC) is being used as a surface layer in more than $85 \%$ of Canada's roadway network. Traffic and environmental loading induces critical levels of stresses in the AC layer. Currently adopted elastic analysis proved less effective for design because of the viscoelastic nature of the $\mathrm{AC}$ response. Mechanistic characterization compatible with emerging mechanistic design models is needed.

This thesis adopted the complex modulus approach to establish a laboratory testing technique for obtaining the parameters needed in mechanistic design. Measured stresses and strains were used to determine the dynamic modulus and phase angle. Effectiveness of the determined parameters was evaluated in two analytical approaches. Database was established using results of tests performed on commonly used AC mixes. The data was organized in a format that fit a variety of applications to function as a pilot material library to serve users with no current testing capabilities.
\end{abstract}




\section{ACKNOWLEDGEMENTS}

I was very fortune in having support, encouragement and advice from several sources. Many thanks for those who gave a hand in accomplishing this work. However, I would like to mention the following specifically for their significant contribution:

My supervisor Professor ElHussein H. Mohamed, for providing me with the opportunity to study this interesting and challenging topic, and for his confident support and advice, which was invaluable source of inspiration. My co-supervisor Professor A. O. Abd El Halim for his valuable support. The examining committee members, Professor S. Vanapalli of Ottawa University, Professor J. Beddoes of Carleton University, Professor S. Sivathayalan of Carleton University, and Mr. R. C. Blockley from the faculty of Graduate studies, Carleton University

Many thanks to the Civil Aviation Authority (CAA) of Sudan, my employer, for providing me with a chance to pursue this graduate program and for the financial support. The National Research Council Canada (NRC) for making their laboratory facilities available for an important component of this program and for the financial support. The City of Ottawa for providing me with the opportunity to use their laboratory facilities to perform conventional laboratory experiments. My appreciation goes for all NRC staff for their support during the period I spent in the Montreal Road Campus. Special thanks to Mr. O. Maadani, Mr. F. Abushoglin, Dr. Morched Zeghal, Dr. Walaa Khogali, Dr. N. Bencharif, and Dr. M. Nofal for their advice and technical support.

Many thanks to all others who were not mentioned in this list but provided support in accomplishing my work.

Finally my gratitude for my family, My wife Shahrzad, and my daughters Muram and Malaz for their patience and sacrifice. My Mother, brothers and sisters for their encouragement. 


\section{TABLE OF CONTENTS}

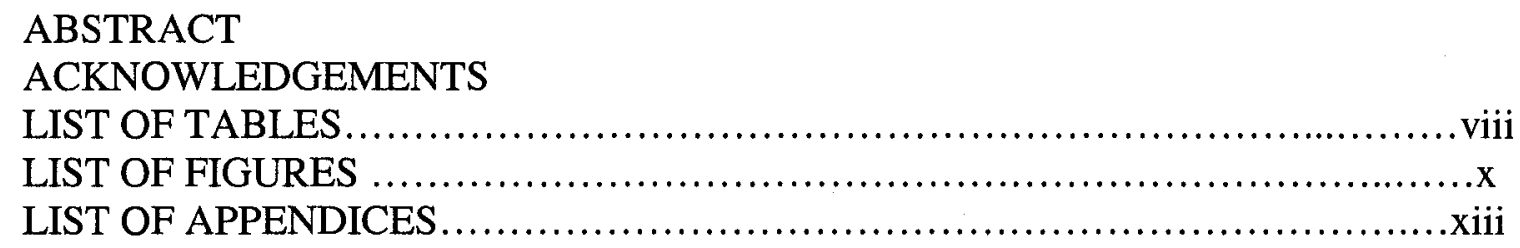

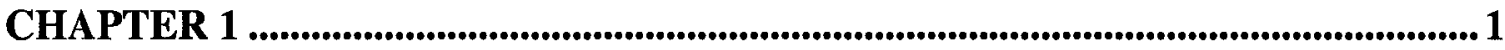

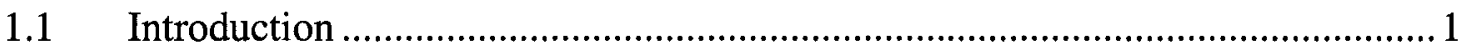

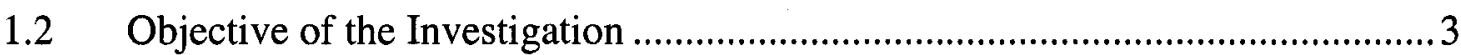

1.3 Scope of the Investigation ........................................................................

$1.4 \quad$ Organization of the thesis..........................................................................

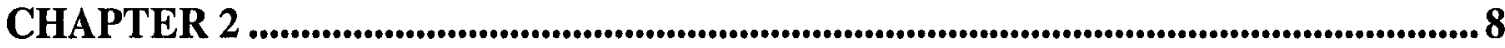

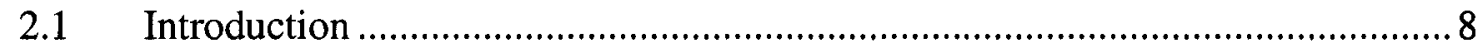

2.2 Pavement Structures ............................................................................... 10

2.3 Conventional Pavement Design Methods ………............................................ 12

2.3.1 AASHTO Pavement Design Method ........................................................13

2.3.2 The Asphalt Institute Method.....................................................................16

2.3.3 National Crushed Stone Association Design Method (NCSA)..................17

2.3.4 California Method of Design................................................................... 18

2.4 Characterization of Asphalt Concrete ………............................................. 19

2.4.1 Marshall Mix Design...........................................................................2 20

2.4.2 SuperPave Mix Design Method …………….........................................22

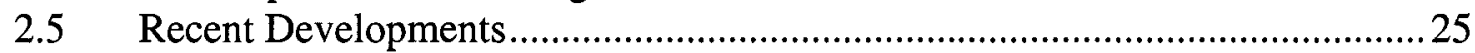

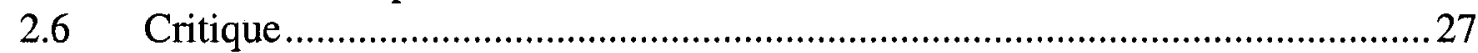

$2.7 \quad$ Research Needs .......................................................................................

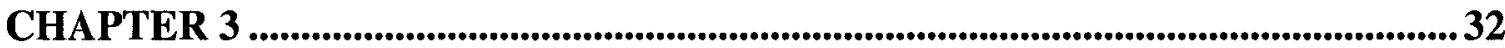

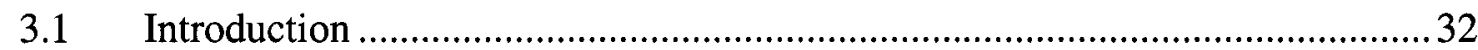

3.2 Mechanistic Characterization of Asphalt Concrete Materials..............................32

3.3 Determination of Linear Viscoelastic Properties ..................................................34

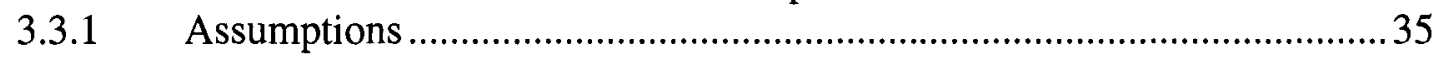

3.3.2 Test and Loading Modes .......................................................................... 35

3.3.3 Determination of Viscoelastic Properties in the Frequency Domain .........36

3.4 Previous Attempts at Characterizing AC Materials .............................................39

3.5 The Complex Modulus Characterization Approach: ...........................................4

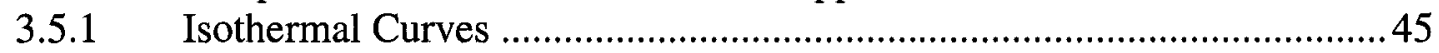

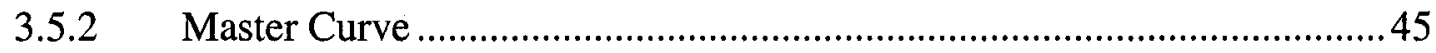

3.5.3 Cole-Cole Diagram .............................................................................4

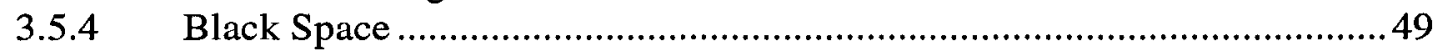

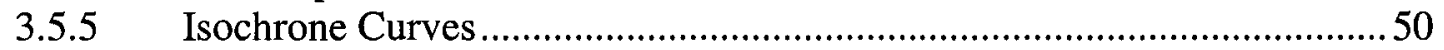

3.6 Applications of the Complex Modulus ...........................................................5 50

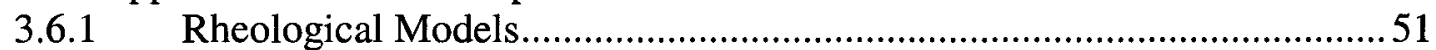

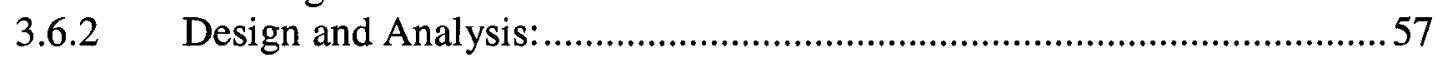

3.6.3 Predictive Equations.............................................................................5

3.6.4 Performance Rating:................................................................................61

CHAPTER 4 ............................................................................................................................................6 63 


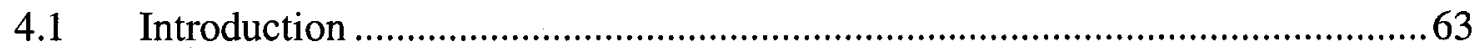

4.2 Development of a Complex Modulus Test Protocol ......................................63

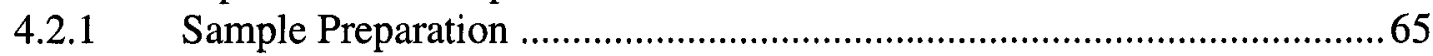

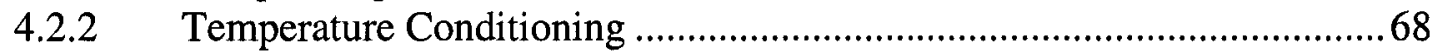

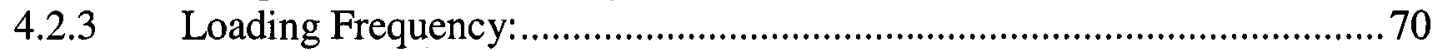

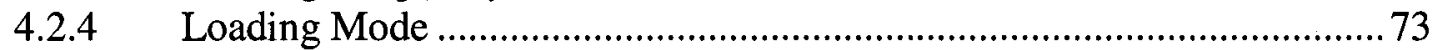

4.2.5 Establishment of the Appropriate Strain Magnitude ..................................73

4.2.6 Test Set-up and Data Acquisition System ............................................. 75

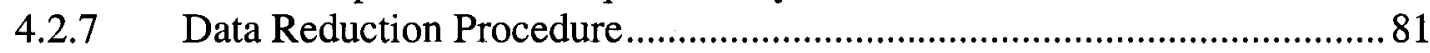

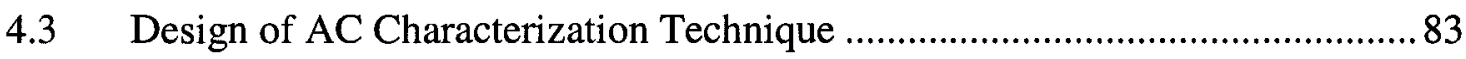

4.3.1 Conventional Physical Characterization of AC Materials ........................ 84

4.3.2 Mechanistic Characterization of AC Materials ........................................96

4.4 Implementations of Complex Modulus Test Output..................................... 102

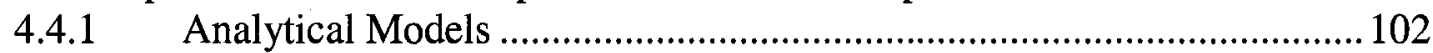

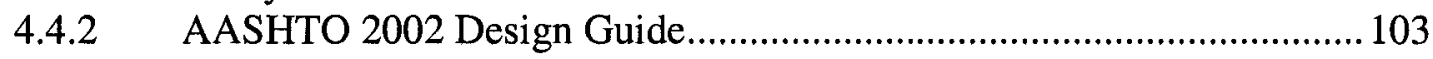

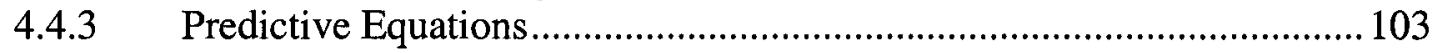

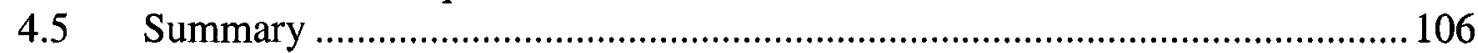

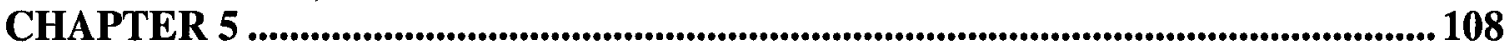

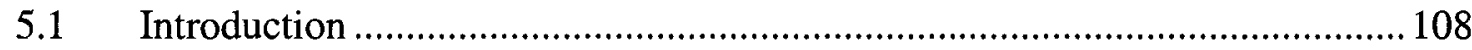

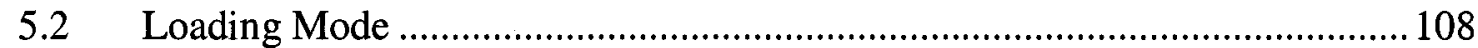

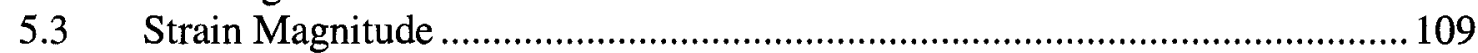

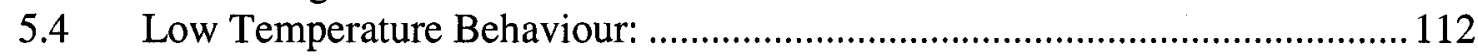

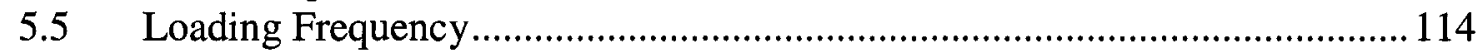

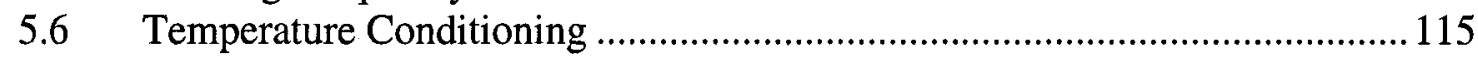

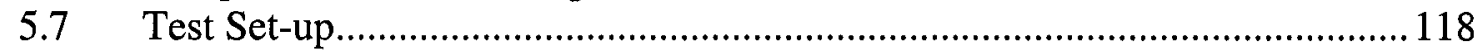

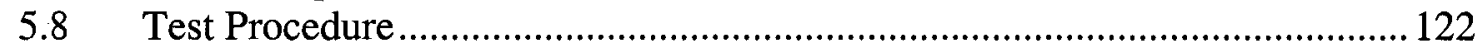

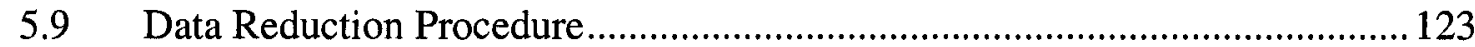

5.10 Characterization Using Processed Data .................................................... 128

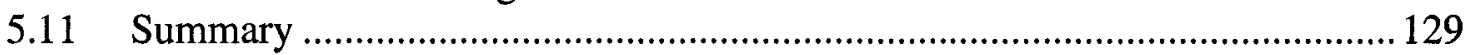

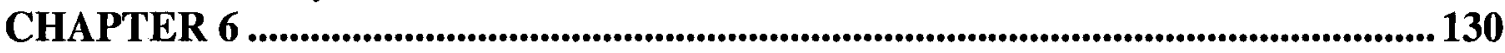

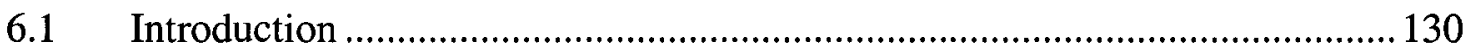

6.2 Determination of Complex Modulus Parameters ........................................ 131

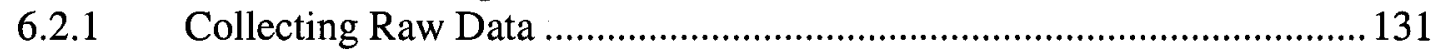

6.2.2 Data Processing Using an Excel-Based Macro ....................................... 132

6.2.3 Refinement of Stress and Strain Signals using "TableCurve" ................ 132

6.3 Assessment of AC Response to Different Factors ....................................... 136

6.3.1 Impact of Traffic Characteristics and Temperature Variations................ 136

6.3.2 Response by Different Mix Types ................................................. 140

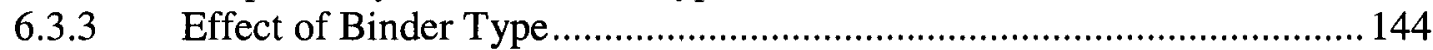

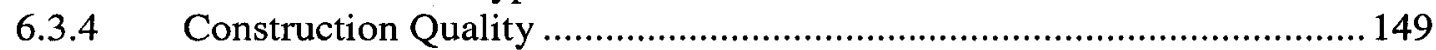

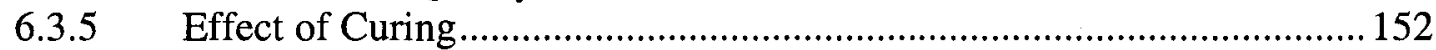

6.3.6 Effect of Mix Design Procedure ......................................................... 156

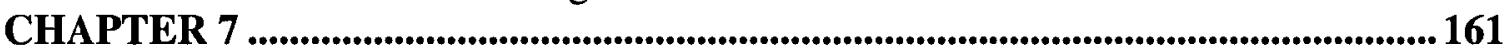

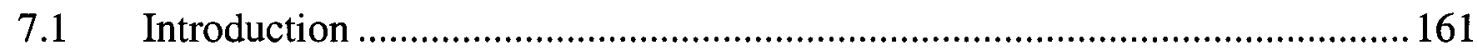

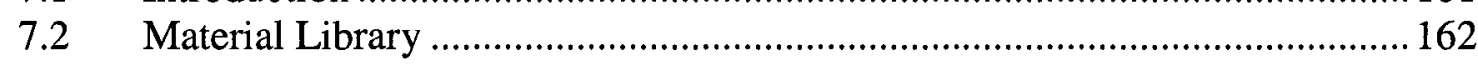

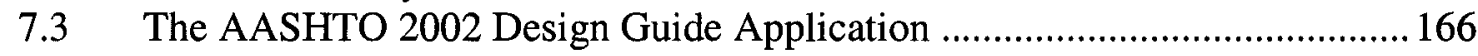




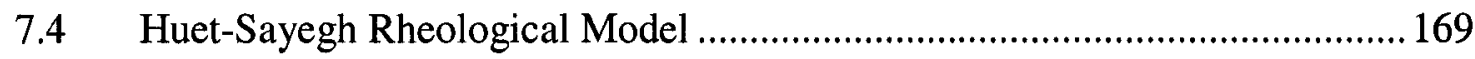

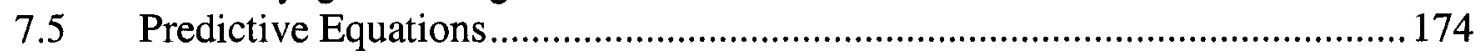

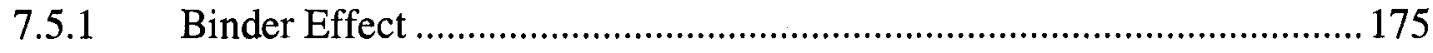

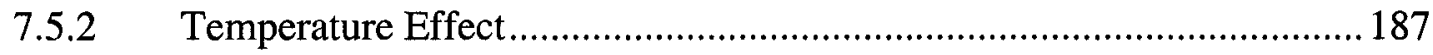

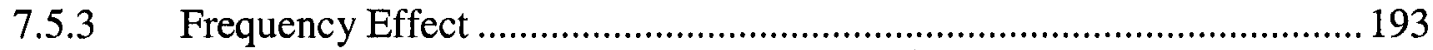

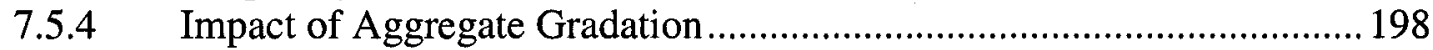

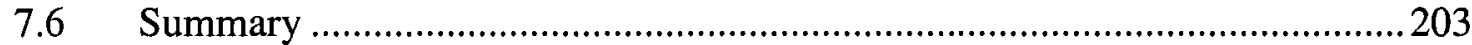

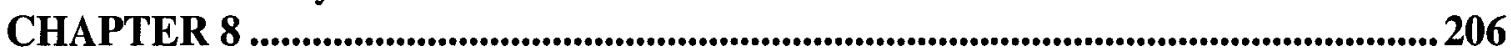

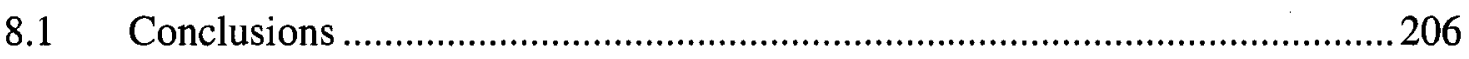

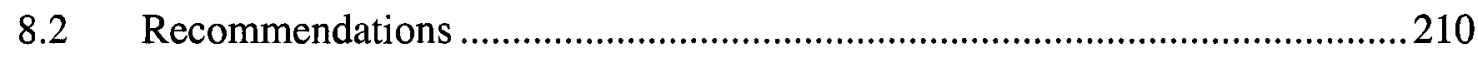

vii 


\section{LIST OF TABLES}

Table 2.1. Usage of AASHTO method in road design across Canada [5] ..........................

Table 2.2. SuperPave Binder Characterization Technique .................................................25

Table 3.1.Test types used to determine asphalt concrete viscoelastic properties [38]......36

Table 3.2. Inventory of temperatures and frequencies used in previous work....................44

Table 3.3. Summary of dynamic modulus predictive equations .........................................58

Table 4.1. Truck matrix with different sizes and weights ................................................72

Table 4.2. Vehicle speeds and corresponding frequencies..............................................72

Table 4.3. Tests designed to check the linearity of the AC response...............................75

Table 4.4. Typical extensometer tuning parameters for $\mathrm{P}$ and I Gains ..............................78

Table 4.5. Number of cycles for all temperature conditions and frequencies....................8 80

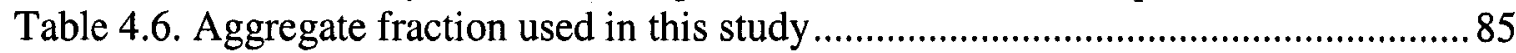

Table 4.7. Results of sieve analysis conducted on six aggregate fractions ........................86

Table 4.8.Binder properties as provided by the manufacturer ...........................................8 87

Table 4.9. Designations of mixes used in this study .....................................................8 88

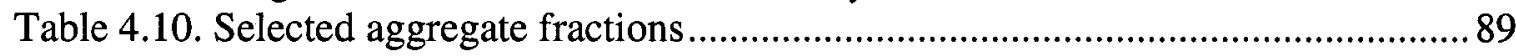

Table 4.11. Marshall mix design results ........................................................................93

Table 4.12. SuperPave mix design results......................................................................93

Table 4.13. List of complex modulus test specimens prepared with binder PG 64-34.....95

Table 4.14. List of complex modulus test specimens prepared with binder PG 58-22 ...95

Table 4.15. List of complex modulus test specimens prepared with binder PG 52-34.....96

Table 4.16. Matrix components of AC characterization .................................................97

Table 4.17. SuperPave mix specimens prepared under different compaction effort .......101

Table 4.18. Physical properties of HMA 3 mix ........................................................... 105

Table 4.19. Physical properties of HMA 2 mix ............................................................... 105

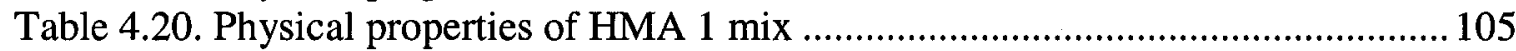

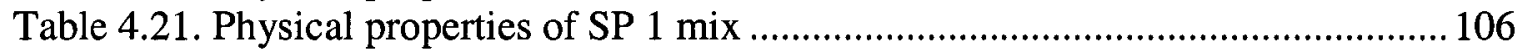

Table 4.22. Physical properties of SP 2 mix ................................................................. 106

Table 5.1. Results of test performed at $-10^{\circ} \mathrm{C}$ using a loading frequency of $10 \mathrm{~Hz}$ to check low temperature behaviour ............................................................................... 113

Table 5.2 Summary of the complex modulus test tasks...................................................124

Table 5.3. Typical acquired raw data ............................................................................. 125

Table 5.4. Data from Table 5.3 processed using a macro .................................................. 126

Table 5.5. Data processed using the "TableCurve"........................................................ 127

Table 5.6. Dynamic modulus and phase angle calculated using output of data processed

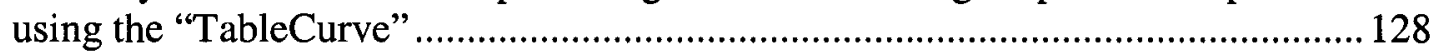

Table 5.7. Typical results of Huet-Sayegh model parameters calculated by the

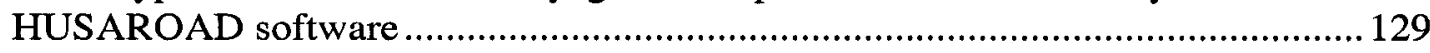

Table 6.1. Factors that influence $\mathrm{AC}$ characteristic response ......................................... 131

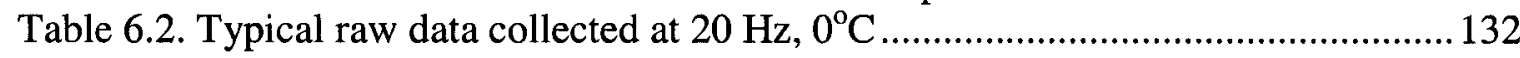

Table 6.3. Processed data using an Excel macro.............................................................. 133

Table 6.4. Stress amplitudes adjusted using "TableCurve" ............................................... 133

Table 6.5. Adjusted stress amplitude as produced by "TableCurve"...............................134

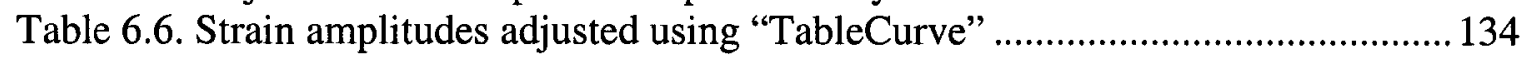

Table 6.7. Adjusted stress amplitude as produced by "TableCurve"................................ 135 
Table 6.8. Stress and strain amplitudes and phase lag calculated by "TableCurve". 135

Table 6.9. Dynamic modulus (MPa) determined at different frequencies and temperatures

Table 6.10. Phase angles $\left({ }^{\circ}\right)$ determined at different frequencies and temperatures....... 137

Table 6.11. Dynamic modulus determined at a constant temperature and different loading frequencies [HMA, PG 58-22]....

Table 6.12. Dynamic modulus determined at a constant loading frequency and different

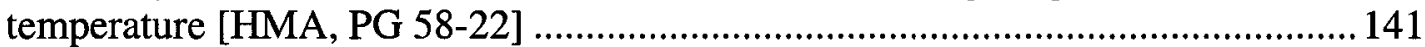

Table 6.13. Dynamic modulus determined at different Temperatures.......................... 145

Table 6.14. Phase angle determined at different Temperatures ................................... 145

Table 6.15. Dynamic modulus (MPa) determined at different air voids $\%$ at 0 and $+30 \mathrm{oC}$

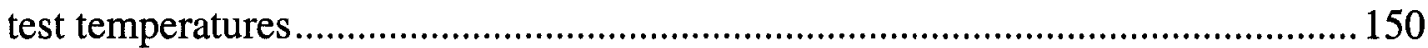

Table 6.16. Phase angles determined at different air voids $\%$ at 0 and $+30 \mathrm{oC}$ test temperatures

Table 6.17. Dynamic modulus (MPa) determined at -10 and $+40^{\circ} \mathrm{C}$ for samples with different ages

Table 6.18. Phase angles $\left({ }^{\circ}\right)$ determined at -10 and $+40^{\circ} \mathrm{C}$ for samples with different ages

Table 6.19. Dynamic modulus for SP 2 and HMA 3 determined at different temperatures using 20 and $0.3 \mathrm{~Hz}$

Table 6.20. Phase angle for SP2 and HMA 3 determined at different temperatures using 20 and $0.3 \mathrm{~Hz}$ 157

Table 7.1. Material library containing typical data pertaining to HMA 1 and SP 2 mixes

Table 7.2. Dynamic moduli (MPa) for the HMA 1 mix (using PG 58-22 binder) ........ 166

Table 7.3. Huet-Sayegh parameters for all mix designs tested in the thesis ................... 174

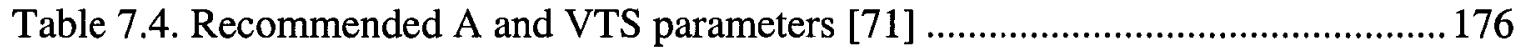

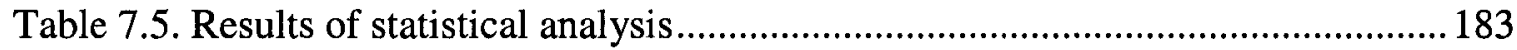

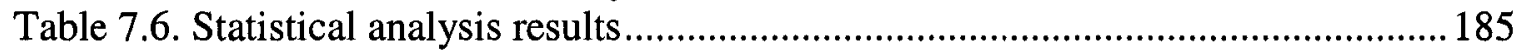

Table 7.7. Results of statistical analysis related to temperature effect.......................... 193

Table 7.8. Statistical analysis results...................................................................... 198

Table 7.9. Results of statistical analysis for Marshall and SuperPave mixes ................203

Table 7.10. Overall evaluation of the 1996 and 2002 equations ...................................2204 


\section{LIST OF FIGURES}

Figure 2.1. Typical components of a flexible pavement [7] …….................................10

Figure 3.1. Phase lag between stress and strain signals .................................................33

Figure 3.2.Typical behaviours observed for bituminous mixtures [38] ..............................33

Figure 3.3. Principles of linear viscoelastic concept ..............................................................37

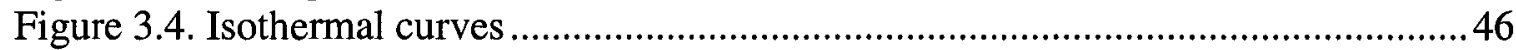

Figure 3.5. Development of a master curve ……………................................................ 48

Figure 3.6. Complex modulus in Cole-Cole plane .......................................................49

Figure 3.7. The complex modulus in Black space ............................................................49

Figure 3.8. Isochrone curves of the complex modulus ..................................................50

Figure 3.9. Schematic representation of the Generalized model.....................................52

Figure 3.10. Schematic representation of Burgers model ...............................................53

Figure 3.11. Schematic representation of the Huet model ................................................54

Figure 3.12. Schematic representation of the Huet-Sayegh models .................................55

Figure 3.13. Complex modulus curves representing the Huet and Huet-Sayegh models in

Cole-Cole plan.....................................................................................................5

Figure 4.1. Design of the experimental investigation .........................................................64

Figure 4.2. a) System used to glue samples, b) handling system details ...........................68

Figure 4.3. Linearity check concept ........................................................................... 74

Figure 4.4. Axial extensometer Model 632.11F-90 ……...........................................76

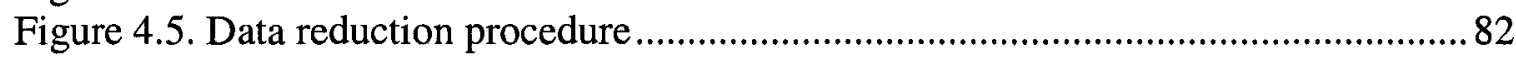

Figure 4.6. Data reduction procedure using TableCurve ....................................................8 84

Figure 4.7.Gradation curve of aggregates used to prepare MHA 1 mix ............................8 89

Figure 4.8.Gradation curve of aggregates used to prepare MHA 2 mix ..............................90

Figure 4.9. Gradation curve of aggregates used to prepare MHA 3 mix ..........................90

Figure 4.10.Gradation curve of aggregates used to prepare SP $1 \mathrm{mix}$.............................91

Figure 4.11.Gradation curve of aggregates used to prepare SP 2 mix ................................91

Figure 4.12.Typical results of HMA3 physical properties.................................................94

Figure 4.13. Typical results of SP 2 physical properties.................................................95

Figure 5.1. Typical stress-strain relationship established at $25^{\circ} \mathrm{C}$ and a loading frequency

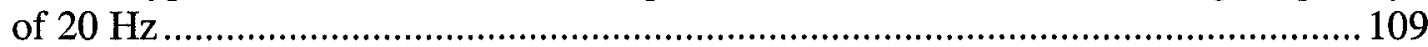

Figure 5.2. Typical results of linearity check based on $(\sigma-\varepsilon)$ relationship $\left[+10^{\circ} \mathrm{C}\right] \ldots .110$

Figure 5.3. Typical results of linearity check using the dynamic modulus $\left[+10^{\circ} \mathrm{C}\right] \ldots \ldots .111$

Figure 5.4. Typical results of linearity check based on $(\sigma-\varepsilon)$ relationship $\left[+25^{\circ} \mathrm{C}\right] \ldots . .111$

Figure 5.5. Typical results of linearity check using the dynamic modulus $\left[+25^{\circ} \mathrm{C}\right] \ldots \ldots .112$

Figure 5.6. (a) Stress-strain relationship, (b) Dynamic modulus-strain relationship for test

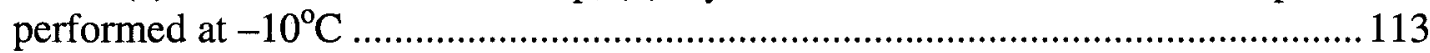

Figure 5.7. Behaviour of $\mathrm{AC}$ materials at low temperatures..........................................115

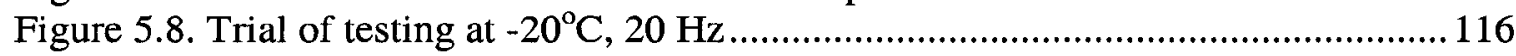

Figure 5.9. Sample broken during a test performed at $-20^{\circ} \mathrm{C}, 20 \mathrm{~Hz} \ldots \ldots \ldots \ldots \ldots \ldots \ldots \ldots \ldots \ldots . .116$

Figure 5.10. Results of trial test at $+50^{\circ} \mathrm{C}$ showing tendency of permanent deformation

Figure 5.11. Strain signal for a test performed using an extensometer not well calibrated

Figure 5.12. Strain signal for an extensometer not well tuned. 
Figure 5.13. Strain signal for adjusted tuning parameters.......................................... 120

Figure 5.14. Complex modulus test set-up................................................................ 121

Figure 5.15. Asphalt concrete specimen ready for testing ........................................ 121

Figure 6.1. Stress and strain signals versus time measured at $20 \mathrm{~Hz}, 0^{\circ} \mathrm{C} \ldots \ldots \ldots \ldots \ldots \ldots . . . . . . .136$

Figure 6.2. Effect of frequency on phase angle [HMA 1, PG 58-22] ...........................139

Figure 6.3. Effect of testing temperature on phase angle [HMA 1, PG 58-22] ..............139

Figure 6.4. Effect of loading frequency on dynamic modulus [HMA 1, PG 58-22] ...... 140

Figure 6.5. Effect of testing temperature on dynamic modulus [HMA 1, PG 58-22].... 140

Figure 6.6. The dynamic modulus determined at different loading frequencies at $0^{\circ} \mathrm{C} . .143$

Figure 6.7. The dynamic modulus determined at different loading frequencies at testing temperature of $+40^{\circ} \mathrm{C}$

Figure 6.8. The dynamic modulus determined at different test temperatures and a loading

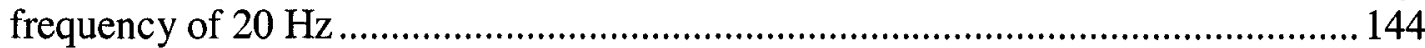

Figure 6.9. The dynamic modulus determined at different test temperatures and a loading frequency of $0.3 \mathrm{~Hz}$................................................................................... 144

Figure 6.10. Dynamic modulus determined at different temperatures and a loading

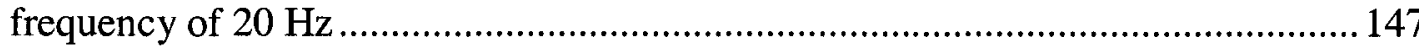

Figure 6.11. Dynamic modulus determined at different temperatures and a loading frequency of $1 \mathrm{~Hz}$

Figure 6.12. Phase angle determined at different temperatures and a loading frequency of $20 \mathrm{~Hz}$

Figure 6.13. Phase angle determined at different temperatures and a loading frequency of $1 \mathrm{~Hz}$

Figure 6.14. Dynamic modulus determined at $0^{\circ} \mathrm{C}$ at different air voids $\%$.................. 151

Figure 6.15. Dynamic modulus determined at $30^{\circ} \mathrm{C}$ at different air voids $\%$................ 151

Figure 6.16. Phase angle determined at $0^{\circ} \mathrm{C}$ and different air voids $\%$......................... 152

Figure 6.17. Phase angle determined at $30^{\circ} \mathrm{C}$ and different air voids $\%$........................ 152

Figure 6.18. Dynamic modulus determined at $-10^{\circ} \mathrm{C}$ after different sample storage periods

Figure 6.19. Dynamic modulus determined at $+40 \mathrm{oC}$ after different sample storage

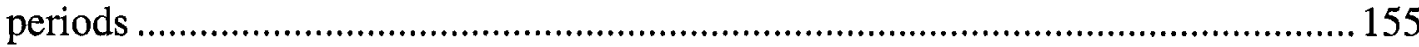

Figure 6.20. Phase angle determined at $-10^{\circ} \mathrm{C}$ after different sample storage periods... 156

Figure 6.21. Phase angle determined at $+40 \mathrm{oC}$ after different sample storage periods .. 156

Figure 6.22. Effect of mix design procedure on dynamic modulus $[0.3 \mathrm{~Hz}]$.................. 158

Figure 6.23. Effect of mix design procedure on dynamic modulus $[20 \mathrm{~Hz}] \ldots \ldots \ldots \ldots \ldots \ldots . . . . . .159$

Figure 6.24. Effect of mix design procedure on phase angle $[20 \mathrm{~Hz}] \ldots \ldots \ldots \ldots \ldots \ldots \ldots \ldots . . . . . . . . . . .159$

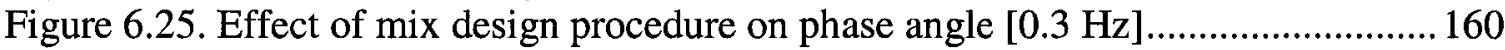

Figure 7.1. Library application in simplified design practices..................................... 163

Figure 7.2. Measured laboratory data for the HMA 3 mix, PG 58-22 ........................ 168

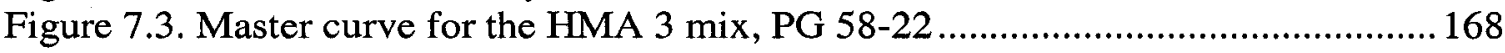

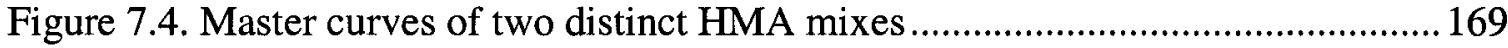

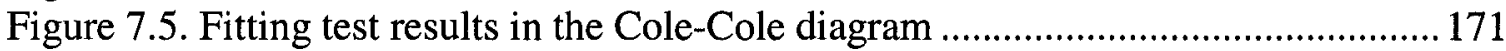

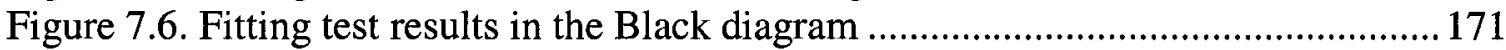

Figure 7.7. Fitting of isothermal curves .............................................................. 172

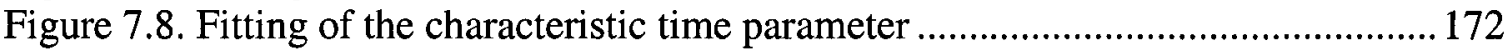

Figure 7.9. Cole-Cole diagram for two HMA mixes ................................................. 173 
Figure 7.10. Cole-Cole diagram for two SuperPave mixes 173

Figure 7.11. Cole-Cole diagram for similar HMA and SuperPave mixes ..................... 174

Figure 7.12. Evaluation of predictions made with 1996 equation for HMA mixes with different high-temperature binder grades

Figure 7.13. Evaluation of predictions made with 1996 equation for SuperPave mixes with different high-temperature binder grades

Figure 7.14. Evaluation of predictions made with 2000 equation for HMA mixes with different high-temperature binder grades

Figure 7.15. Evaluation of predictions made with 2000 equation for SuperPave mixes with different high-temperature binder grades.

Figure 7.16. Predicted dynamic modulus vs. temperature -1996 equation .................... 181

Figure 7.17. Predicted dynamic modulus vs. temperature -2000 equation ..................... 182

Figure 7.18. Measured dynamic modulus vs. temperature ........................................ 182

Figure 7.19. Evaluation of predictions made with 1996 equation for HMA mixes with different low-temperature binder grades

Figure 7.20. Evaluation of predictions made with 2000 equation for HMA mixes with different low-temperature binder grades .......................................................... 184

Figure 7.21. Predicted dynamic modulus vs. temperature -1996 equation ................... 186

Figure 7.22. Predicted dynamic modulus vs. temperature -2000 equation ................... 186

Figure 7.23. Measured dynamic modulus vs. temperature ........................................... 187

Figure 7.24. Dynamic modulus predictions at $-10^{\circ} \mathrm{C}$ using 1996 equation...................... 189

Figure 7.25. Dynamic modulus predictions at $-10^{\circ} \mathrm{C}$ using 2000 equation...................... 189

Figure 7.26. Dynamic modulus predictions at $+20^{\circ} \mathrm{C}$ using 1996 equation.................... 190

Figure 7.27. Dynamic modulus predictions at $+20^{\circ} \mathrm{C}$ using 2000 equation.................... 190

Figure 7.28. Dynamic modulus predictions at $+40^{\circ} \mathrm{C}$ using 1996 equation.................... 191

Figure 7.29. Dynamic modulus predictions at $+40^{\circ} \mathrm{C}$ using 2000 equation................... 192

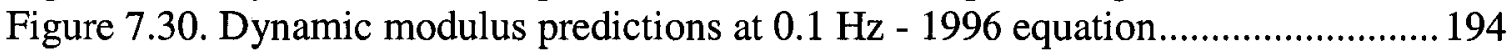

Figure 7.31. Dynamic modulus predictions at $0.1 \mathrm{~Hz}-2000$ equation.......................... 195

Figure 7.32. Dynamic modulus predictions at $1 \mathrm{~Hz}-1996$ equation ............................ 195

Figure 7.33. Dynamic modulus predictions at $1 \mathrm{~Hz}-2000$ equation............................. 196

Figure 7.34. Dynamic modulus predictions at $20 \mathrm{~Hz}-1996$ equation............................ 196

Figure 7.35. Dynamic modulus predictions at $20 \mathrm{~Hz}-2000$ equation........................... 197

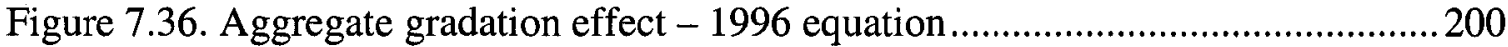

Figure 7.37. Aggregate gradation effect -1996 equation.............................................200

Figure 7.38. Aggregate gradation effect -2000 equation ........................................... 201

Figure 7.39. Aggregate gradation effect -2000 equation ........................................... 201

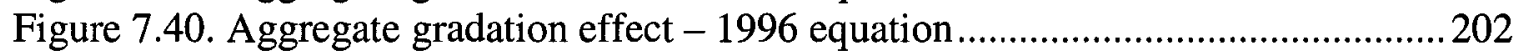

Figure 7.41. Aggregate gradation effect -2000 equation .......................................202

Figure 7.42 Comparison between predictions made with 1996 and 2000 equations...... 205 


\section{LIST OF APPENDICES}

Appendix A: Complex Modulus Test Protocol and Data Reduction Procedure............. 213

Appendix B: Asphalt Concrete Material Library ................................................. 227

Appendix C: Macro for Reducing Complex Modulus Test Data..................................2238

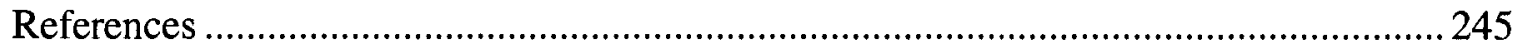

xiii 


\section{CHAPTER 1 \\ CHARACTERIZATION OF ASPHALT CONCRETE}

\subsection{Introduction}

Considerable resources are dedicated annually worldwide towards the maintenance and development of urban infrastructure, including roads, bridges and buried utilities. The annual budget for urban roads alone exceeds $40 \%$ of total municipal expenditures on infrastructure. Canadian municipalities spend about $\$ 8$ billion annually on urban roads [1]. Similar amounts are spent by provincial governments in highway construction. A major portion of these resources $(85 \%)$ are directed toward road repairs. Consequently, this situation reduces the opportunity for new developments that may enhance the level of performance, reduce traffic congestion and improve safety measures. With many other key services such as health and education competing for available public funds, the road industry is seeking cost-saving measures to reduce the cost of repair and rehabilitation so that remainder of funds can be used for road development activities.

Urban roads and highways have a significant impact on national economic activities. They play an important role in determining the living standards of the nation. This significance can be seen clearly in the continuous increase in both number and size of vehicles that use the roadway networks, both those used by passengers and those used to transport goods.

The recent trend in the increase of traffic volume and vehicle weight indicates that roads are being subjected to higher loads than in the past. This means more damage, suggesting 
the need for more attention to be given to improving road designs to meet this increase in demand and to maintain an adequate level of performance.

Roads are multilayered structures, with asphalt concrete acting as the surface layer in more than $90 \%$ of urban roads and highways nationwide. The asphalt concrete is the layer in which all types of damage are manifested. Therefore, proper design of this layer is an effective approach for confronting damage associated with the growth in vehicle numbers and weights.

Numerous studies reported that an asphalt concrete overlay is one of the most common techniques of pavement rehabilitation in North America [2]. However, continued rapid road deterioration following a major rehabilitation project still represents the most critical problem facing highway engineers and administrators today. The durability of asphalt concrete layer represents an important cost issue and a challenge for researchers as well as road authorities. The Transportation Association of Canada (TAC), the national body with a mandate to address such a problem, decided in 1989 to join the Strategic Highway Research Program (SHRP) of the United States. Accordingly, C-SHRP was formed, followed by the Canadian Long-Term Pavements Performance (C-LTPP) program. The C-LTPP was established to pursue the development of measures to increase the service life of pavements through the development of cost-effective pavement rehabilitation procedures [3]. The adopted research approach was based solely on systematic observation of the performance of in-service test sections constructed with asphalt concrete, which were designed according to the conventional Marshall Mix design procedure and the new SHRP product referred to as the Superior Performing Asphalt Pavement (SuperPave). No definitive recommendations emerged from this initiative for 
improving the cost effectiveness of road rehabilitation based on overlays. The only recommendation that was endorsed by the Canadian road industry relates to the new asphalt concrete characterization technique, which was adopted by binder manufacturers who compete in the North American market.

This thesis examined the connection between the properties of asphalt concrete and its components and current structural pavement design procedures, but found no adequate link. The establishment of such a material-mechanics link will facilitate the implementation of effective design procedures to support the move towards performancebased road design and rehabilitation measures including overlays.

\subsection{Objective of the Investigation}

The objective of the research work performed in this thesis was to investigate asphalt concrete characterization techniques and proceed to develop an approach that is compatible with evolving mechanistic pavement design procedures. The implementation of the new technique will enable engineers to use emerging road mechanistic design methodologies capable of producing durable and cost-effective rehabilitation solutions.

\subsection{Scope of the Investigation}

The scope of the experimental investigation reported in this thesis included a number of tasks as described below:

1. Reviewed the literature and examined the ability of current material characterization techniques in supporting mechanistic road design procedures.

2. Selected a material characterization procedure entitled the "Complex Modulus" for its effectiveness and suitability for analytical modelling of road structures involving 
asphalt concrete layers. The choice of the complex modulus was also made in anticipation of its incorporation into emerging pavement design models.

3. Designed a test procedure for determining the complex modulus parameters, taking into consideration material rheological behaviour in the field. The developed test protocol and the established characterization technique were evaluated to assess their sensitivity to elements that are known to influence the characteristic response of asphalt concrete including the following:

- Temperature sensitivity of asphalt concrete, which significantly influences the material response within the different seasons.

- Traffic load characteristics, which in combination with the temperature effect, influences the viscous response of the material. Accordingly, a wide range of loading frequencies and test temperatures were included in the laboratory investigation covering critical traffic speeds and temperatures that simulate cold and warm conditions.

4. Investigated the behaviour of a number of conventional and new North American mix designs focusing on:

- Conventional Marshall mix design, which is the most commonly used technique for hot mix asphalt (HMA) in Canada.

- SuperPave mix design, which is a relatively gap-graded mix design developed by the Strategic Highway Research Program (SHRP) initiative. This mix design procedure is expected to replace the Marshall mix design in the near future. 
Investigated the impact of construction quality on the mechanistic response in the laboratory using the developed testing protocol performed on samples prepared using different levels of compaction because it influences the integrity of the material placed in the field

Prepared a database using the characteristic response determined for the mixes tested in this thesis, together with conventional physical properties. The database will assist users, who do not have the facilities to perform the complex modulus test, to start using the newly developed mechanistic models benefiting from the results incorporated in the "Material Library" produced in this study.

Model developers, such as those responsible for the ASSHTO 2002 Design Guide, are aware of the complexity of mechanistic testing techniques and data reduction requirements. Predictive equations were developed for determining the dynamic modulus using physical properties. The determined modulus will then be used at a lower (simplified) design level. The predictive equations were evaluated in this thesis to determine their accuracy. This component of the thesis is important because the majority of users of the proposed design guide will prefer to use this level until they establish testing capabilities similar to the one developed in this thesis.

\subsection{Organization of the thesis}

This thesis was organized according to the scope of the investigation discussed earlier:

Chapter 1: Provides a general introduction describing the status of road infrastructure and presents the objective and the scope of the thesis. 
Chapter 2: Discusses the state-of-the-art in pavement design, focusing on the absence of a link between current design procedures and the mechanistic response of asphalt concrete mixes. Emerging road designs and analysis techniques were discussed and the need for a mechanistic characterization technique identified.

Chapter 3: Presents the potential for using the complex modulus technique in characterizing $\mathrm{AC}$ mixes and its effectiveness in providing necessary material input to analytical models. Discussions in this chapter include earlier European and North American attempts to apply the complex modulus approach. The chapter also contains a review of the theoretical concept supporting the test approach.

Chapter 4: Describes the experimental investigation designed to establish the complex modulus testing protocol, the new characterization technique applied to common $\mathrm{AC}$ mixes and experiments performed to evaluate the sensitivity of the developed characterization technique.

Chapter 5: Analyzes the data collected from tests designed in Chapter 4 related to the development of the test protocol to highlight the confronted issues and discuss the solutions adopted to arrive at the final test version.

Chapter 6: Discusses the results of tests obtained from examining various AC mix types, starting with data reduction followed by analysis of data collected for the various studies identified in Chapter 4.

Chapter 7: Discusses implementation techniques proposed for applying findings of this thesis, including the new $\mathrm{AC}$ characterization technique. Emphasis was placed on evaluating the validity of a recently developed predictive equation proposed for 
application in a simplified design technique included in the AASHTO 2002 Design Guide.

Chapter 8: Summarizes the principal findings of the thesis and recommendations made for follow-up studies.

Appendices, including (A) the developed test protocol, (B) a macro developed for data reduction and $(\mathrm{C})$ the Material Library consisting of the properties of $\mathrm{AC}$ mixes characterized according to the approach developed in this thesis. 


\section{CHAPTER 2 \\ STATE-OF-THE ART}

\subsection{Introduction}

Asphalt concrete is the most commonly used material for paving road surfaces and overlaying deteriorated road sections [2]. It is the layer that exposed to the full effect of tire pressure and other types of external forces applied by traffic and elements of the environment. The asphalt concrete surface course is designed to provide adequate skid resistance and to prevent the penetration of surface water into the underlying unbound layers. Withstanding tire pressures as high as $690 \mathrm{kPa}(100 \mathrm{psi})$ or even more, abrasive forces due to vehicle movements (braking and turning), and thermally induced stresses require special attention during the design process to prevent rapid rates of deterioration and consequent reduced road serviceability.

The current dilemma facing pavement engineers results from the fact that available analytical tools are not capable of predicting the impact of the external loading on the performance of the road structure, especially the AC layer. The theoretical construct of today's analytical models used in design and analysis has been limited to the knowledge obtained from the AASHO Road Test results of the early 1960s. The road test was limited in scope and results are only reliable for the conditions established during the AASHO Road Test (one subgrade soil condition and limited spectrum of traffic loads).

In spite of their reduced budgets under current fiscal constraints on public spending, Canadian municipalities are still forced to dedicate a considerable portion of their budgets towards rehabilitation of roads to confront an alarming rate of deterioration. A survey conducted by the Urban Infrastructure Program of the National Research Council of 
Canada (NRC), revealed that rehabilitated roads deteriorate substantially after only 5 to 9 years [4]. The design life of these rehabilitated roads shown in the books by most road agencies are 15 to 20 years as shown in Table 2.1 [5]. Current design and analysis tools are unsuccessful in preventing premature cracking and rutting responsible for the observed short service life.

Researchers and transportation agencies are looking forward to a mechanistic design and analysis approach in an attempt to predict the potential for unfavourable rates of deterioration before implementing proposed rehabilitation measures [6]. If implemented, such an approach is expected to produce effective design solutions and a variety of costeffective rehabilitation alternatives. The literature search that highlights limitations in current design tools is summarized in the following sections of this chapter with emphasis on deficiencies in the characterization of pavement materials that made the link with the mechanics of the road structure not possible.

Table 2.1. Usage of AASHTO method in road design across Canada [5]

\begin{tabular}{|c|c|c|}
\hline Province/Agency & General Design Method(s) & $\begin{array}{l}\text { Design Life (year) } \\
\text { New/Rehabilitation }\end{array}$ \\
\hline British Columbia & AASHTO 93 & $20 /-$ \\
\hline Alberta & - $\quad$ AASHTO 93 & $20 / 20$ \\
\hline Saskatchewan & $\begin{array}{ll} & \text { Shell } \\
- & \text { Asphalt Institute } \\
\end{array}$ & $15 / 15$ \\
\hline Manitoba & $\begin{array}{r}-\quad \text { AASHTO } 93 \text { (new construction) } \\
-\quad \text { Asphalt Institute (rehabilitation) }\end{array}$ & $20 / 20$ \\
\hline Ontario & $\begin{array}{ll}\text { - } & \text { AASHTO 93 (Adapted for local } \\
\text { conditions) } \\
\text { - } & \text { Asphalt Institute } \\
\text { - } & \text { Ontario Standards } \\
\end{array}$ & $20 / 20$ \\
\hline Quebec & - $\quad$ AASHTO 93 & $\begin{array}{l}\text { Major highway: } 20 / 20 \\
\text { Other projects: } 15 / 15\end{array}$ \\
\hline New Brunswick & - $\quad$ AASHTO 93 & $20 / 15$ \\
\hline Prince Edward Island & - Asphalt Institute & $20 / 12$ \\
\hline Nova Scotia & $\begin{array}{ll}- & \text { AASHTO } 93 \\
\text { - } & \text { Correlating Charts using AADT \& } \\
& \text { grain size of subgrade }\end{array}$ & $20 /--$ \\
\hline Newfoundland & - $\quad$ Standard section used & -- \\
\hline PWGSC* & $\begin{array}{ll}- & \text { AASHTO } 93 \\
- & \text { State of Alaska Design Method } \\
\end{array}$ & $20 / 12$ \\
\hline
\end{tabular}

*PWGSC: Public Works and Government Services Canada 


\subsection{Pavement Structures}

Conventionally, pavements are classified into two categories, rigid and flexible. Flexible pavements may consist of a relatively thin asphalt concrete wearing surface built over a granular base course and subbase course, with both resting on a compacted subgrade. On the other hand, rigid pavements are made up of Portland cement concrete and may or may not have a base course between the pavement and the subgrade [7]. The focus of this thesis, however, is on flexible pavements, which represent more than $90 \%$ of the Canadian roadway network. Figure 2.1 shows a typical flexible pavement structure.

The essential difference between rigid and flexible pavements is in the manner in which the load is distributed over the subgrade. Because of its rigidity and high modulus of elasticity, rigid pavements tend to distribute the load over a relatively wider area of soil as compared to flexible pavements, and thus the slab itself supplies a major portion of the structural capacity.

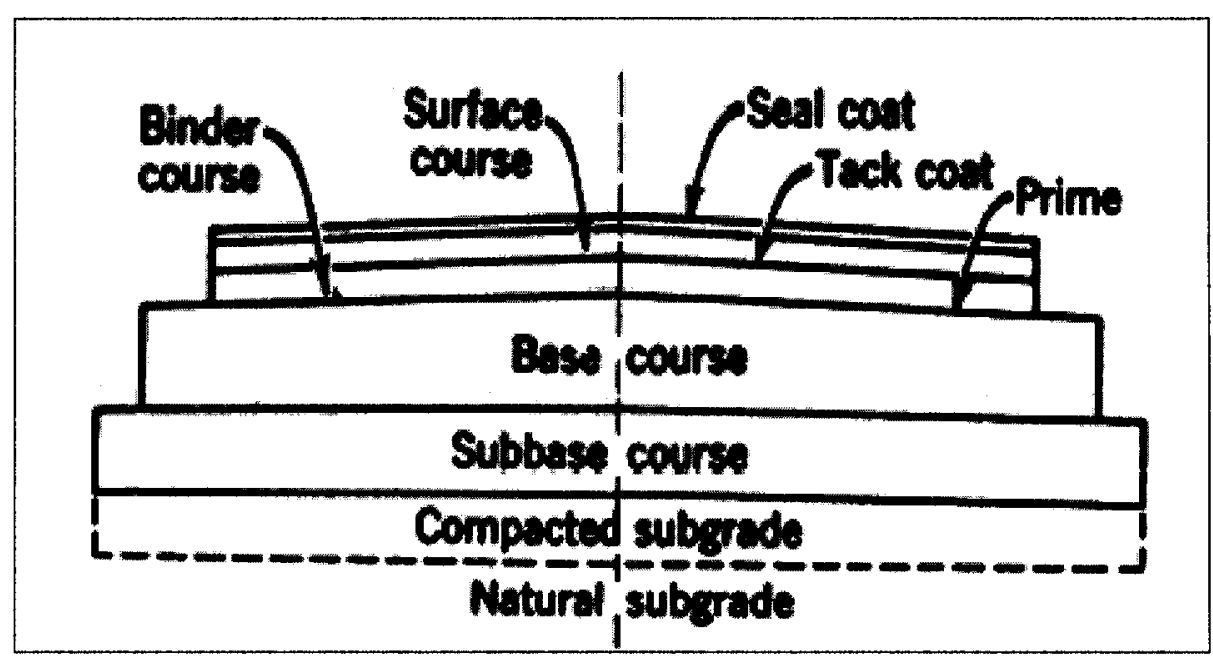

Figure 2.1. Typical components of a flexible pavement [7] 
Pavement structures are exposed to two distinct types of failure [7]:

(a) Structural failure: Includes a collapse or breakdown of one or more of the layers, which makes the pavement incapable of sustaining the loads imposed on the surface.

(b) Functional failure: In which the pavement may barely carry out its intended function resulting in discomfort to vehicle passengers and/or damage to the vehicle itself. Such a failure mode occurs as a consequence of increased surface roughness.

Pavement failures may take many forms including $[8,9$,$] :$

- Loss of material, such as that caused by ravelling, which leads to surface roughness or a localized failure such as potholes.

- A discontinuity in the form of cracks or opened joints along the longitudinal or transverse directions of the pavement. A number of cracking mechanisms are identified in the literature including fatigue and thermal cracking. The progressive nature of this type of distress may cause intensive cracking [10], leading to an unstable driving surface, or to faulting, where migration of surface moisture into the pavement foundation results in deformation causing an uneven road surface.

- Deformations associated with the AC layers, which may take the form of lateral road profile changes (waves) or wheel path rutting in the longitudinal direction. This type of distress is caused by one or a combination of deformation mechanisms [11]:

(a) Volume change associated with densification of the material (void closure);

(b) Shear flow where the binder and fine material forming the $\mathrm{AC}$ mix move under vehicle wheel passes. 
Attempts were made in the past to reduce the impact of these damage mechanisms during the AC mix design or pavement design, or by specifying measures to be followed during construction. Such attempts included in current practices are discussed in the following sections.

This thesis focuses on promoting analytical models capable of tracing the response of the AC layer to facilitate the prediction of evolving damage patterns.

\subsection{Conventional Pavement Design Methods}

The structural design of pavements is different from that of other structures such as bridges and buildings. Pavement structures directly rest upon the ground surface and pass across a variety of soils. Hence, they are affected to a great extent by the characteristics of these soils, including density, moisture content, texture, structure and mineralogical composition. The response of the native soil and borrowed materials forming the road base is stress dependent; meanwhile, the response of the $\mathrm{AC}$ material to external loading is time dependent. These factors together with other factors such as the variation of soil characteristics from point to point, the random nature of traffic and environmental variables, make the pavement design process very complex [12].

There are several different flexible pavement design methods currently in use. The most commonly adopted ones in Canada include the AASHTO, the Asphalt Institute (TAI), the National Crushed Stone Association (NCSA), and the California Methods. A summary of these design methods is given below. 


\subsubsection{AASHTO Pavement Design Method}

Historically, the AASHTO pavement design method has undergone frequent changes. Upon completion of the AASHO Road Test in the late 1950s and early 1960s in Ottawa, Illinois, an "AASHO - 1961 Interim Guide for the Design of Rigid and Flexible Pavement" [13] was developed and circulated to the transportation agencies in North America. Pavement design procedures were developed based on the test results and design criteria.

Two main concepts were introduced in the AASHO 1961 Interim Guide; the serviceability and performance. The serviceability was defined as the ability of a pavement to serve the traffic for which it was designed. Meanwhile, performance was defined as the ability of the pavement to serve traffic over a period of time. Serviceability ratings from 0 to 5 were obtained by taking the mean ratings performed by a group of experts, where 0 indicates poor pavement and 5 indicates excellent pavement.

Equation 2.1 was developed based on findings of the AASHO Road Test to reflect the flexible pavement design approach [13].

$\log W_{t 18}=9.36 \log (S N+1)-0.2+\frac{\log \left[\left(4.2-p_{t}\right) /(4.2-1.5)\right]}{0.4+\left[1094 /(S N+1)^{5.19}\right]}$

where

$W_{t I 8}=$ the number of 18-kip single axle load applications to time $t$,

$p_{\mathrm{t}} \quad=$ serviceability at the end of time $t$, and

$S N \quad=$ Structural Number of pavement 
However, after several years of practice, Equation 2.1 was found not to be applicable for climatic conditions and soil types that are different from the ones encountered at the time the original Road Test was completed. Accordingly, the design formula was modified to include the effect of the climate and the soil type by introducing a regional climatic factor (R) and a subgrade soil support value $\left(\mathrm{S}_{\mathrm{i}}\right)$. The modified design equation took the form shown in Equation 2.2, which was included in the new "AASHTO 1972 Interim Guide for the Design of Pavement Structures" [14].

$$
\log \mathrm{W}_{\mathrm{t} 18}=9.36 \log (\mathrm{SN}+1)-0.2+\frac{\log \left[\left(4.2-\mathrm{p}_{\mathrm{t}}\right) /(4.2-1.5)\right]}{0.4+\left[1094 /(\mathrm{SN}+1)^{5.19}\right]}+\log \frac{1}{\mathrm{R}}+0.372\left(\mathrm{~S}_{\mathrm{i}}-3.0\right)
$$

In 1983, an evaluation of the Interim Guide was made in light of new information developed since 1972 to improve the pavement design process. Several changes were introduced in the 1983 revised version of the AASHTO Guide. Accordingly, design Equation 2.2 was modified to the following [15]:

$$
\log \mathrm{W}_{\mathrm{t} 18}=\mathrm{Z}_{\mathrm{R}} \bullet \mathrm{S}_{0}+9.36 \log (\mathrm{SN}+1)-0.2+\frac{\log [(\Delta \mathrm{PSI}) /(4.2-1.5)]}{0.4+\left[1094 /(\mathrm{SN}+1)^{5.19}\right]}+2.32 \log \mathrm{M}_{\mathrm{R}}-8.07
$$

where

$Z_{\mathrm{R}}=$ Standard normal deviation,

$\mathrm{S}_{0} \quad=$ Combined standard error of the traffic prediction and performance prediction

$\triangle P S I=$ Difference between the initial design serviceability index, $p_{o}$, and the design terminal serviceability index, $p_{t}$, and

$\mathrm{M}_{\mathrm{R}}=$ Resilient modulus (psi) 
The soil support value $\left(\mathrm{S}_{\mathrm{i}}\right)$ in Equation 2.2 has been replaced with the resilient modulus, $M_{R}$, which represents the first time a mechanistic material response is included in the analysis and design of roads. However, including the $M_{R}$ parameter served only in characterizing unbound material layers while the $\mathrm{AC}$ layer, an integral component of the road structure, remained neglected.

It is worth mentioning that the modified equations are predictors of the amount of sustainable traffic before the road deteriorates to some terminal level of serviceability. However, Van der Poel [16] has advocated that since the pavement is a layered structure, each layer must be checked to ensure that an adequate thickness of the material is provided for in the design. With the absence of an appropriate representation of the $\mathrm{AC}$ material in the design equation, such a check was not possible for this specific layer.

In 1993, the AASHTO Guide was updated again. However, the modifications this time took place in the specifications of rigid pavements only by introducing drainage factors. Flexible pavement specifications remained unchanged [17].

The AASHTO design guide is virtually used by all road authorities in North America except for a few states and provinces. Table 2.1 shows the usage of the 1993 version of the Design Guide across Canada [5]. Information provided in Table 2.1 indicates that the majority of Canadian agencies use the AASHTO design procedure either as a primary design tool or to verify designs completed using an alternative methodology. However, the AASHTO Design Guide remains not sensitive for analysis of the AC layer and hence provides unreliable design solutions for recurring problems such as wheel path rutting. 


\subsubsection{The Asphalt Institute Method}

This procedure is based primarily on analysis of the performance of the asphalt concrete layers in test sections of the AASHO Road Test. Thus, indirectly, the adequacy of the performance - serviceability concept is reflected in design solutions obtained from the AI method. The AI procedure is mainly concerned with estimating the thickness of the equivalent full-depth AC layer of the pavement structure considering the effect of traffic and subgrade strength. The basic formula developed by the Asphalt Institute is shown in Equation 2.4 [18].

$T=a_{0}+a_{1} \log W+a_{2} L_{1}+a_{3} L_{1} L_{2}$

where $\mathrm{T}=$ thickness index,

$$
\begin{aligned}
& \mathrm{W}=\text { load applications to } p=2.5, \\
& \mathrm{~L}_{1}=\text { single-axle load in kips or one-half the tandem-axle load in kips, } \\
& \mathrm{L}_{2}=\text { a code number equal to } 0 \text { for single axles and } 1 \text { for tandem axles } \\
& \mathrm{a}_{0}, \mathrm{a}_{1}, \mathrm{a}_{2}, \mathrm{a}_{3}=\text { regression constants }
\end{aligned}
$$

The thickness index is a mathematical expression used to combine the varying combination and thickness of surface, base and subbase layers into a single parameter. The expression for $\mathrm{T}$, in terms of an equivalent granular base thickness, is given by Equation 2.5.

$\mathrm{T}_{\mathrm{G}}=1.0 \mathrm{D}_{1}+\mathrm{D}_{2}+0.75 \mathrm{D}_{3}$

where $D_{1}, D_{2}, D_{3}$ represent the thickness of the surface, base, and subbase layers respectively. Using the AASHO Road test data and applying an adjustment to include 
$97 \%$ of the data points to arrive at a conservative design estimate led to the development of the thickness Equation 2.6. This equation expresses the design thickness in the form of an equivalent asphalt concrete thickness.

$\mathrm{T}_{\mathrm{A}}=-10.10+2.75 \log \mathrm{W}+0.325 \mathrm{~L}$

where $T_{A}$ is the equivalent total asphalt concrete layer thickness and $W$ and $L$ are as defined previously. It should be noted that Equation 2.6 represents the design equation for conditions applicable only to those of the original AASHO Road test. Consequently, a need to extend the use of the equation to include other regions with different subgrade conditions rises. Considering the effect of traffic in terms of a Design Traffic Number (DTN) and the effect of variable subgrade strength, represented by the California Bearing Ration (CBR), a modified thickness design equation of the form displayed in Equation 2.7 was suggested by the Asphalt Institute [18].

$$
\mathrm{T}_{\mathrm{A}}=\frac{9.19+3.971 \log \mathrm{DTN}}{\mathrm{CBR}^{0.4}}
$$

Although Equation 2.7 relates the thickness of the asphalt concrete layer to traffic and some physical properties of the subgrade layer, it remains unresponsive to typical material behaviour (asphalt concrete is a viscous material with a response that depends largely on prevailing temperature conditions), and consequently, it does not satisfy mechanistic design and analysis requirements.

\subsubsection{National Crushed Stone Association Design Method (NCSA)}

The National Crushed Stone Association design method is based upon the US Army Corps of Engineers CBR method of pavement design. The objective of this procedure is 
to provide adequate thickness and quality of material to prevent repetitive shear deformation within any layer. Additionally, measures to minimize the effects of frost action to tolerable levels were incorporated into the design process [19].

The NCSA method was intended to circumvent dependency on layer equivalency and substitution ratios of the AASHTO and the Asphalt Institute pavement design methods. The use of such unique empirical coefficients and substitution ratios for design ignores the following:

- The position of the material within the pavement structure, which determines the magnitude of traffic-induced stresses at that position. Unbound materials response, for example, is stress dependent and ignoring such a factor will produce overdesigned or under-designed solutions.

- Material type

- Construction quality

- In-situ conditions

- Characteristics of traffic loading

However, as indicated in the NCSA charts, reliance on material physical properties to arrive at the appropriate design thickness will not capture accurately the mechanistic response that governs the pavement performance.

\subsubsection{California Method of Design}

This method is totally empirical; stabilometer values (or R-values for short) are used along with material equivalency factors to design the pavement structure to withstand the 
effect of traffic expressed as a traffic index parameter (TI). Field performance records were collected from road surveys, which were then utilized to develop the design Equation 2.8. Application of this equation involves the determination of the required thickness above each of the materials used in the various layers of the pavement structure [20].

$\mathrm{GE}=0.0032(\mathrm{TI})(100-\mathrm{R})$ 2.8

where

$\mathrm{GE}=$ gravel equivalent

$\mathrm{TI}=$ traffic index, and

$\mathrm{R}=$ stabilometer value

As evident from the formulation of Equation 2.8, the link between the actual material response and the obtained design thickness is missing.

\subsection{Characterization of Asphalt Concrete}

Previous gaps in knowledge regarding the principles of mechanics needed to construct a theoretically sound model for pavement design and analysis dictated reliance on empirical procedures similar to those discussed in Section 2.3. These empirical design procedures were based on some indices, and at best, on physical properties to characterize the different materials used in building the road. This approach was applied to asphalt concrete as well where the mix design process is used to produce a material with adequate resistance to known forms of damage, mainly rutting.

The process described above is completely in isolation from the structural design process, which is supposed to consider the characteristic response of the material in the analysis. 
Two of the most commonly used mix design procedures are discussed below, namely; (a) the conventional Marshall mix design and (b) the SuperPave procedure, which is a North American initiative that was developed as part of the Strategic Highway Research Program (SHRP) in 1995 [21].

\subsubsection{Marshall Mix Design}

The Marshall mix design procedure was developed in the late 1930's. It is the most widely used design technique worldwide. The procedure follows certain steps to produce uniform asphalt mixtures including the following [22]:

- Aggregate selection: Aggregates meeting general requirements for durability (abrasion, stiffness, etc.) are selected.

- Gradation: Sieve analysis is performed to produce a combination of aggregate fractions which, when considered, will satisfy specifications. These specifications were developed based on experience and field trials.

- Selection of a design binder content: the amount of asphalt cement to be used as binder is determined based on past experience and field trials. The binder content is needed to coat aggregates and produce the cohesion required to hold them together, hence providing the flexibility needed under traffic action. The optimum binder content is determined as the amount that will not result in bleeding.

- Mixing: Mechanical mixing is used to perform this step and produce a homogeneous mix. Because of the nature of commonly used binders, high temperatures are needed to reduce the viscosity of the material to facilitate the coating of mineral aggregates during this process. 
- Compaction: Compaction level (number of hammer blows) is specified according to the anticipated traffic level. The automatic Marshall hammer is used to apply 25,50 , and 75 blows for low, medium, and high levels of traffic, respectively.

- Mix design characteristics: Laboratory-prepared samples are subjected to physical tests intended to evaluate mix criteria including the following:

1- Bulk specific gravity and density as specified in AASHTO Designation: T166 $[23]$.

2- Air Voids (\%): Air pockets between coated aggregates in compacted sample as determined by AASHTO Designation: T228 [24].

3- Voids in Mineral Aggregates (VMA): It is the volume of the intergranular void space between the aggregate particles of the compacted paving mixture that includes the air voids and the effective binder content, expressed as a percent of the total volume of the specimen.

4- Voids Filled with Asphalt (VFA): Represents the percentage of the VMA filled with binder (the effective binder volume divided by the VMA).

5- Marshall Stability $(\mathrm{kN})$ and flow $(\mathrm{mm})$ : Determined as specified in AASHTO T 245 [25]. Stability and flow are used as measures for resistance to plastic flow of bituminous mixtures.

The Marshall method benefits from a set of physical characteristics of the mix to arrive at the intended material quality during construction. Stability and flow tests were the only attempts to achieve a semi-mechanical measure. However, there are limitations associated with the stability and flow tests because of their empirical nature. For instance, Monismith [26] indicated that stability is just a number and is not compatible with 
analytically based procedures; nevertheless, Kandhal et al. [27] have advocated that at least it provides some measure of the mix quality. The Marshall stability and flow parameters are still in use today for specifying AC properties and specifications.

\subsubsection{SuperPave Mix Design Method}

The SuperPave mix design method is a product of the Strategic Highway Research Program (SHRP) [28]. The goal of this initiative was to develop an effective mix design procedure including the establishment of sustainable aggregate gradation and performance-based binder specifications. The new mix design procedure was intended to overcome problems associated with asphalt pavements by specifying binder types capable of offering adequate resistance to rutting, fatigue and low-temperature cracking [29].

The SuperPave approach was basically built on the knowledge acquired from applying the Marshall method. However, the primary differences between the two procedures are the laboratory compaction method used to prepare test specimens and the strength tests used to evaluate the mixes.

Researchers found that the gyratory compactor, used in the SuperPave procedure, produces mixes that simulate the field mix better compared with mixes produced by the Marshall hammer [30]. This assertion was confirmed by Fonseca and Witczak [31], who remarked that the gyratory method of compaction is ideal as it produces an aggregate structure similar to that obtained in situ including both the initial compaction and repeated trafficking patterns. Many other researchers also investigated the different methods of compaction available today [32 and 33]. 
There are three levels of the SuperPave mix design. The Level $1 \mathrm{mix}$ design is basically a volumetric procedure and does not include mechanistic characterization of the mix. Volumetric properties (air voids, asphalt content, etc.) and aggregate properties (crushed faces, fine aggregate sharpness, etc.) are the basis of Level 1. In some ways, the Level 1 mix design is similar to the Marshall mix design.

Both Level 2 and Level 3 use results of mechanistic tests as performance indicators. The main SuperPave evaluation technique involves a shear test. However, these mechanical tests are not receiving the support of users.

In summary, using physical properties of the asphalt concrete mix and correlating them to performance has been predominant in the Marshall mix design method. The SuperPave procedure, on the other hand, benefited from the results of some mechanistic tests to predict performance. However, no analytical model has yet been established to benefit from these test results in the analysis of road structures. The key output of the SHRP Project was a set of new specifications for asphalt binders now known as performance grade binders $(\mathrm{PG})$. The following section highlights the main features of the new binder specification system developed as part of the SuperPave project.

\section{Performance-Grade Binder Specifications}

The current practice of asphalt specifications was developed based on physical properties such as penetration, ductility, and viscosity. However, several drawbacks have been identified in this system. Current tests are empirical, (i.e., pavement performance experience is required to enable a meaningful interpretation of the test results.) Another limitation is that the physical tests mentioned above do not provide enough information for the entire range of typical pavement temperatures. For example, a standard test 
temperature of $25^{\circ} \mathrm{C}$ is selected to describe the penetration grade, while temperatures ranging from 60 to $135^{\circ} \mathrm{C}$ are used to determine the viscosity [18]. It is obvious that cold temperature effects are ignored.

Binder specification was one of three major components of the SHRP project 37-A1, entitled "The SuperPave." Recognizing the deficiencies in the current specification system, the SuperPave developed a new set of tests to measure physical properties that can be related directly to field performance using engineering principles. Table 2.2 lists the equipment required to conduct the new tests together with a brief description of the usage and the output(s) of each test [18].

The tests shown in Table 2.2 are used to quantify the asphalt performance at three stages of its life including the initial state, after mixing and construction, and the final in-service state. Some of these test parameters were initially intended as a characterization technique that would be implemented in modeling the entire $\mathrm{AC}$ mix. The ratio $\mathrm{G} * / \sin \delta$ was selected as a rutting factor, and $\mathrm{G}^{*} \sin \delta$ to be a fatigue cracking factor. However, the high cost of the equipment dedicated to perform these tests stood in the way of applying the SuperPave mechanistic characterization. Only the performance-based binder specifications, referred to as Performance-Grade (PG), were adopted by the industry.

A unique feature of specifying asphalt binders by their Performance-Grade designations is that the required physical properties remain constant for all performance grades. However, the temperatures at which these properties must be achieved may vary. For example, consider two regions, cold and warm: good performance is expected in both cases, but the temperatures under which the specified binder properties must be achieved are vastly different. The PG specification method is now being used in Canada to 
determine the suitability of a binder type in performing adequately in certain environmental conditions. For instance, PG 58-22 performs better in an environment with temperatures up to $+58^{\circ} \mathrm{C}$ and down to $-22^{\circ} \mathrm{C}$.

Table 2.2. SuperPave Binder Characterization Technique

\begin{tabular}{|c|c|c|}
\hline Test & Purpose & Output \\
\hline $\begin{array}{l}\text { Rolling Thin Film Oven } \\
\text { (RTFO) }\end{array}$ & $\begin{array}{l}\text { Simulate binder aging that } \\
\text { occurs during mixing and } \\
\text { construction }\end{array}$ & \multirow{2}{*}{$\begin{array}{l}\text { Quantity of volatiles lost from } \\
\text { binder (mm), which is an } \\
\text { indication of the aging that } \\
\text { may occur in the asphalt } \\
\text { during mixing and } \\
\text { construction process }\end{array}$} \\
\hline $\begin{array}{l}\text { Pressure Aging Vessel } \\
\text { (PAV) }\end{array}$ & $\begin{array}{l}\text { Simulate the in-service } \\
\text { aging of binder }\end{array}$ & \\
\hline $\begin{array}{l}\text { Dynamic Shear Rheometer } \\
\text { (DSR) }\end{array}$ & $\begin{array}{l}\text { Measure binder properties } \\
\text { at high and intermediate } \\
\text { temperatures }\end{array}$ & $\begin{array}{l}\text { The complex shear modulus } \\
\left(G^{*}\right) \text { and the phase angle }(\delta) \\
\text { of asphalt binder }\end{array}$ \\
\hline Rotational Viscometer (RV) & $\begin{array}{l}\text { Measure binder properties } \\
\text { at high temperatures }\end{array}$ & $\begin{array}{l}\text { Viscosity of asphalt binder at } \\
135^{\circ} \mathrm{C} \text { and } 165^{\circ} \mathrm{C}\end{array}$ \\
\hline $\begin{array}{l}\text { Bending Beam Rheometer } \\
\text { (BBR) }\end{array}$ & $\begin{array}{l}\text { Measure binder properties } \\
\text { at low temperatures }\end{array}$ & $\begin{array}{l}\text { Creep stiffness } S(t) \text { of asphalt } \\
\text { binder, and } m \text {-value, which } \\
\text { represents the rate of change } \\
\text { in creep stiffness }\end{array}$ \\
\hline $\begin{array}{l}\text { Direct Tension Tester } \\
\text { (DTT) }\end{array}$ & $\begin{array}{l}\text { Measure the amount of } \\
\text { binder strain before } \\
\text { failure at low } \\
\text { temperatures }\end{array}$ & Failure strain \\
\hline
\end{tabular}

\subsection{Recent Developments}

Because of the empirical nature of previous ASHHTO design procedures, the AASHTO Design Guide was examined in the late 1990s with the intention of pursuing a mechanistic-empirical model with the ability to support road rehabilitation tasks. The Project entitled "AASHTO 2002 Design Guide" was initiated to update the design procedure. Many difficulties were encountered and the design guide is currently under evaluation; it is expected to be completed in 2005 (three years after the target date). Difficulties associated with the AC component of the model pushed the model more 
towards the empirical side of analysis. The latest version of the model is expected to adopt two new material characterization techniques for pavement design purposes. The first of these techniques advocates the use of the Resilient Modulus $\left(M_{R}\right)$ as a means for characterizing the mechanistic response of unbound granular materials, while the second calls for the use of the Dynamic Modulus $\left|\mathrm{E}^{*}\right|$ to characterize the mechanistic response of asphalt concrete. A hierarchical approach is proposed with regard to traffic, materials, and environmental inputs and in some cases to the types of analyses used. There are three major levels of design as described below:

Level 1: Level 1 is an advanced design procedure and provides for the highest, practically achievable level of reliability. The design inputs require site-specific data collection and/or testing. Examples are dynamic modulus testing of asphalt concrete and site-specific axle load spectra.

Level 2: Level 2 is the standard design procedure to be used for routine design. Level 2 inputs typically would be user selected, possibly from an agency database, derived from a less than optimum testing program. They may also be estimated empirically. Examples would be the dynamic modulus estimated from binder, aggregate, and mix properties, or site-specific traffic volume and classification data used in conjunction with agencyspecific axle load spectra.

Level 3: Level 3 typically is the lowest class of design and would be used where there are minimal consequences of early failure and on lower volume roads. Inputs typically would be user-selected default values. Examples would be default dynamic modulus values for given mix classes or default axle load spectra for functional highway classes. 
It can be noticed that the dynamic modulus $\left|E^{*}\right|$ is incorporated in all hierarchical levels of the mechanistic-empirical approach adopted by the 2002 Design Guide. This reflects the significance of this parameter as a material property that can be used in the future in developing reliable models for pavement design and analysis.

\subsection{Critique}

A review of current pavement design procedures and mix design methods revealed that the two tasks are performed independently. Asphalt concrete characterization, performed as part of the mix design task, is based mainly on physical properties. Pavement design procedures up to the current AASHTO-1993 Design Guide, used by almost all provinces, are primarily empirical in nature and still rely on the results of the AASHO Road Test performed in the early 1960 s.

Current pavement design and analysis models cannot be used to predict performance, taking into consideration the type of mix used or quality of construction achieved in the field. In summary:

- Most methods focus on determining the thickness of the pavement layers based on prior observed performance, such as the one obtained from the results of the AASHO Road Test. Characteristic responses of materials making up the pavement structure, the thickness of the AC layer itself, the condition of the native soil (subgrade), drainage considerations, and other factors are ignored.

- Apparently, frequent updates of the AASHTO design guide target the overcoming of limitations resulting from the absence of a link between the design procedure and material properties required for characterization of the mechanistic response of the 
pavement layer constructed with the material in question. The absence of such a link motivated the author of this thesis to pursue a new mechanistic-based characterization technique to support future development of effective pavement design and analysis tools.

- The literature review revealed that current design methods focus on the subgrade strength while ignoring other critical layers of the pavement, namely the asphalt concrete and the granular base. The simplified current design approach, which is based primarily on subgrade support, overlooks the fact that critical levels of trafficinduced stresses prevail in these layers with minimum levels reaching the subgrade. Accordingly, in a performance-based design procedure, the asphalt concrete and unbound granular layers should be accounted for.

- Current design methods apply equivalent load factors to account for the impact of traffic on performance. The use of a single factor ignores the impact of other weight characteristics, which vary for different types of truck. A simple load factor, developed primarily from repeated wheel load application data of an arbitrarily selected standard truck, should not be expected to cover the wide variations in weight parameters such as tire pressure, contact area, sprung and un-sprung weight, axle distribution and changes in weight associated with braking and turning manoeuvres (critical for urban streets).

- The extent of accumulated deformation depends also on the level of stress and the number of load repetitions (traffic volume). Nevertheless, the stress was never incorporated into any of the design methods discussed earlier. Moreover, the influence of temperature was not addressed explicitly in any of the previous versions 
of the design guide. Van Til et al. [34] evaluated the AASTHO Interim Design Guide application in the U.S. and found that only five states use temperature data to establish the Regional factor (R). The remainder majority of the states still use engineering judgment. Pavement temperature is a significant factor that influences the performance of asphalt concrete pavements. Relatively high temperatures reduce the stiffness of AC layer, thus making it more susceptible to deformations. On the other hand, relatively low temperatures (less than $5^{\circ} \mathrm{C}$ ) make the $\mathrm{AC}$ layer extremely brittle and consequently more susceptible to cracking.

- The current AC mechanical tests are mainly strength tests, which were included in the mix design methods as potential performance predictors. However, the results of these tests cannot be applied directly in mechanistic design models. Marshall stability is a number that is unreliable for its empirical nature, while the shear complex modulus of the SuperPave has not been implemented in any of the emerging models. Moreover, the test itself is considered expensive and its relation to performance has not yet been verified.

- It seems that the complexity associated with developing an effective characterization technique for the materials used in the base and surface layers, delayed the development of an effective design approach for roads. However, with recent advances in mechanics and material science, and evolving computational capabilities, it is anticipated that the switch to a more sound structural design approach employing material properties that better describe the characteristic response of the various pavement layers, will be greatly facilitated. The attempt made by ASSHTO (2002 Design Guide) [35] and the Minnesota DOT [36] to develop Mn/DOT flexible 
pavement design are key examples of the new direction the industry in pursuing to improve road design and analysis.

- Early signs indicate that the dominance of the empirical components of the proposed ASSHTO guide may reduce its effectiveness in addressing some of the critical issues currently facing road designers. An example of this empiricism is the extensive use of predictive equations to produce rheological properties of $\mathrm{AC}$ materials as will be discussed in Chapter 7.

\subsection{Research Needs}

Developing procedures to define requisite material characteristics is necessary for establishing analytically based design methodologies such as those employing stress and strain solutions. In the context of pavement design, measuring the stiffness characteristics of the various pavement materials used in the different layers is critical for successful implementation of such analytical models.

Attempts to characterize AC materials can be classified under two categories:

1. Physical properties determination: These properties, discussed earlier under the Marshall and SuperPave mix design procedures, include aggregate size and distribution (gradation), air voids, bulk specific gravity and density. Based on current knowledge, these parameters alone are not suitable for mechanistic design methods.

2. Mechanical properties determination: These properties include the Marshall Stability and Flow numbers, the shear modulus of SuperPave and the indirect tensile strength used in some current practices. As mentioned earlier, these properties proved less effective and are not included in the new analytical models currently being 
established for pavement design and analysis. Shortcomings associated with accurately characterizing the $\mathrm{AC}$ response or the high cost associated with performing the necessary tests also rendered these tests impractical.

Attempts to use the Young's modulus were unsuccessful since the actual material response includes viscous and plastic components. Early analytical models, based on linear elastic theory, provided reasonable results only under certain conditions (low temperatures and high traffic speeds). However, these models have failed in analyzing pavement behaviour observed in the field and in the laboratory involving medium and high temperature conditions, and also roads with medium to low traffic speed such as urban streets. These conditions invoke viscoelastic and plastic responses, which are responsible for pavement deformations that manifest as wheel path rutting.

This thesis proposes a new technique for $\mathrm{AC}$ characterization that accounts for the viscoelastic behaviour of this material. The proposed technique is based on the determination of the complex modulus parameters as discussed in the following chapter. 


\section{CHAPTER 3 \\ MECHANISTIC CHARCTERIZATION TECHNIQUES}

\subsection{Introduction}

This chapter discusses the complex modulus approach to the characterization of asphalt concrete, which is capable of producing mechanistic properties that can be used as input in analytical models. Included also is a survey of experimental and theoretical concepts that support the complex modulus approach. A literature search was conducted and previous attempts to characterize AC materials mechanistically were reviewed to highlight the evolution of the test parameters along with factors influencing the complex modulus of AC materials. Moreover, this chapter discusses the conventional methods used to reduce data and present results of the complex modulus test.

\subsection{Mechanistic Characterization of Asphalt Concrete Materials}

As discussed in Chapter 2, characterization of asphalt concrete materials based mainly on physical properties hampered the success of analytical tools intended for the support of design and analysis of road pavements. A rational characterization technique should facilitate capturing major elements of the mechanistic response under prevailing traffic and environmental conditions. Measurements performed in the Netherlands with a linear tracking apparatus (LINTRACK) in a field experiment using accelerated loading capabilities indicated that there is a time retardation effect associated with the longitudinal strain in asphalt concrete. The maximum strain developed only after the load had passed the strain gauge as depicted in Figure 3.1. The signal measured by LINTRACK [37] indicated that the longitudinal strain shows compression first, then tension, and then compression again. This response is typical of asphalt concrete 
behaviour and is known to reflect the dependency of the response on time, confirming its viscous nature.

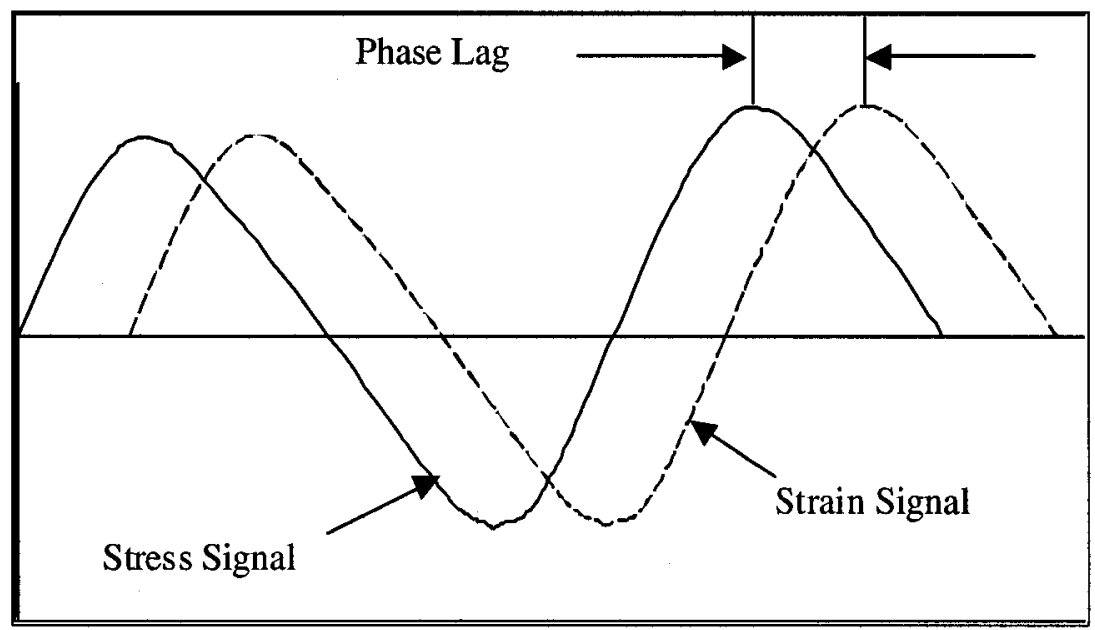

Figure 3.1. Phase lag between stress and strain signals

Di Benedetto [38] indicated that the strain amplitude and the number of loading cycles can be used to classify the behaviour of bituminous mixtures into three typical modes as shown in Figure 3.2. At small values of strain $\left(<10^{-4}\right)$ and a few hundred load cycles the behaviour can be best described as linear viscoelastic.

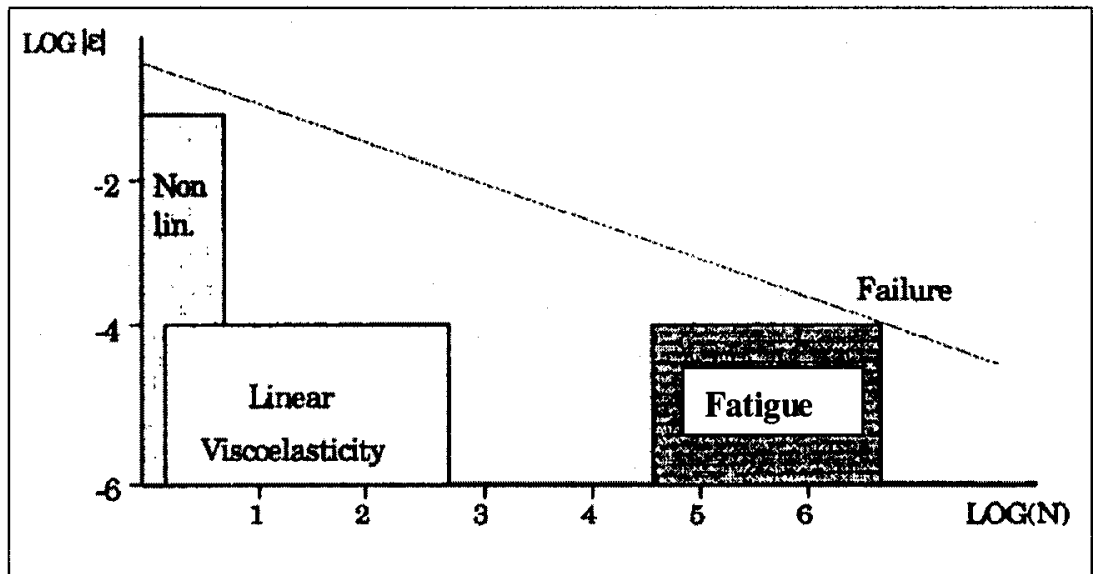

Figure 3.2.Typical behaviours observed for bituminous mixtures [38] 
The response of bituminous mixtures (much like the binder it contains) is significantly affected by temperature and loading frequency $[11,39]$. Their response to a given loading is strongly dependent on temperature as well as the rate of load applications, known as frequency. Monismith [26] concluded that at temperatures above $25^{\circ} \mathrm{C}$, the stress has an influence on the stiffness characteristics as the binder becomes less stiff. Heck et al. [37] and Monismith [26] indicated that because of the viscoelastic nature and thermal susceptibility of asphalt concretes; the stiffness characteristics of mixtures are dependent on traffic velocity and temperature. Therefore, the frequency-temperature couple has to be mentioned whenever stiffness of asphaltic material is mentioned. The mechanistic characterization of asphalt mixtures that takes into account the above-mentioned elements could be best achieved by measuring properties that describe their linear viscoelastic behaviour. The linear viscoelastic theory has the advantage of allowing the use of BOLTZMANN superposition principles in which the response of a material under a load made up of a certain number of elementary loads is equal to the sum of the responses under each of these elementary loads [40].

\subsection{Determination of Linear Viscoelastic Properties}

As discussion made in section 3.2 , viscoelastic properties are required to characterize $\mathrm{AC}$ materials. A number of studies in the literature investigated the viscoelastic response of these materials $[40,41,42,43$, and 44$]$. The following subsections discuss some of the techniques that investigated the application of viscoelastic principals in the analysis of the AC response. 


\subsubsection{Assumptions}

The proposed AC characterization technique benefited from the theoretical construct of the viscoelastic theory because of its good representation of $\mathrm{AC}$ behaviour in the field under repetitive loading and exposure to a wide range of pavement temperatures. The analytical solution to the state of stress or strain in the adopted approach relies on some assumptions meant to simplify analysis. These assumptions include the following [7]:

- The material properties of each layer are homogeneous, i.e., the property at two different points within the same layer is similar. However, in practice, heterogeneity is dominant;

- Each layer has a finite thickness, and all are infinite in the lateral directions; that is to eliminate the lateral shear effect. In other words, shearing forces are not present at the surface;

- Each layer is isotropic, i.e., the property at a specific point is the same in every directions or orientation. Isotropy is an assumption made in spite of the anisotropy introduced when $\mathrm{AC}$ layers are laid in the field compacted by successive passes of compactors.

\subsubsection{Test and Loading Modes}

In the application of the linear viscoelastic theory, many types of loads can be used. However, the temporal and frequency domains are the most common loading modes. In the time domain, a quasi-static load is usually applied while a sinusoidal load was found to be more suitable for the frequency domain. Two types of testing techniques are 
available for determining the AC viscoelastic properties, homogenous and nonhomogenous tests [38].

Measurements of viscoelastic properties of $\mathrm{AC}$ materials require a homogenous type of testing in which stresses and strains are directly accessed. Non-homogenous tests are more complicated and necessitate rigorous calculations and postulation of constitutive laws. Non-homogenous tests are suitable only for capturing simple behaviour such as those associated with a linear elastic response. The test types evaluated in the campaign of the International Union of Testing and Research Laboratories for Materials and Structures (RILEM) focusing on the development of performance testing and evaluation of bituminous materials are listed in Table 3.1 according to the appropriate category [38 and 45].

Table 3.1.Test types used to determine asphalt concrete viscoelastic properties [38]

\begin{tabular}{|l|l|}
\hline Homogenous Tests & Non-Homogenous Tests \\
\hline Tension-Compression test & 2 points bending \\
\hline Shearing test & 3 points bending \\
\hline Constant height shearing test & 4 points bending \\
\hline Co-axial shearing test & Indirect Tensile test \\
\hline
\end{tabular}

\subsubsection{Determination of Viscoelastic Properties in the Frequency Domain}

As the name indicates, viscoelastic properties of $\mathrm{AC}$ materials are usually determined within the linear viscoelastic domain. Within this domain, the response of an $\mathrm{AC}$ material to sinusoidal loading is also sinusoidal but with a phase lag [42] as shown in Figure 3.3. 

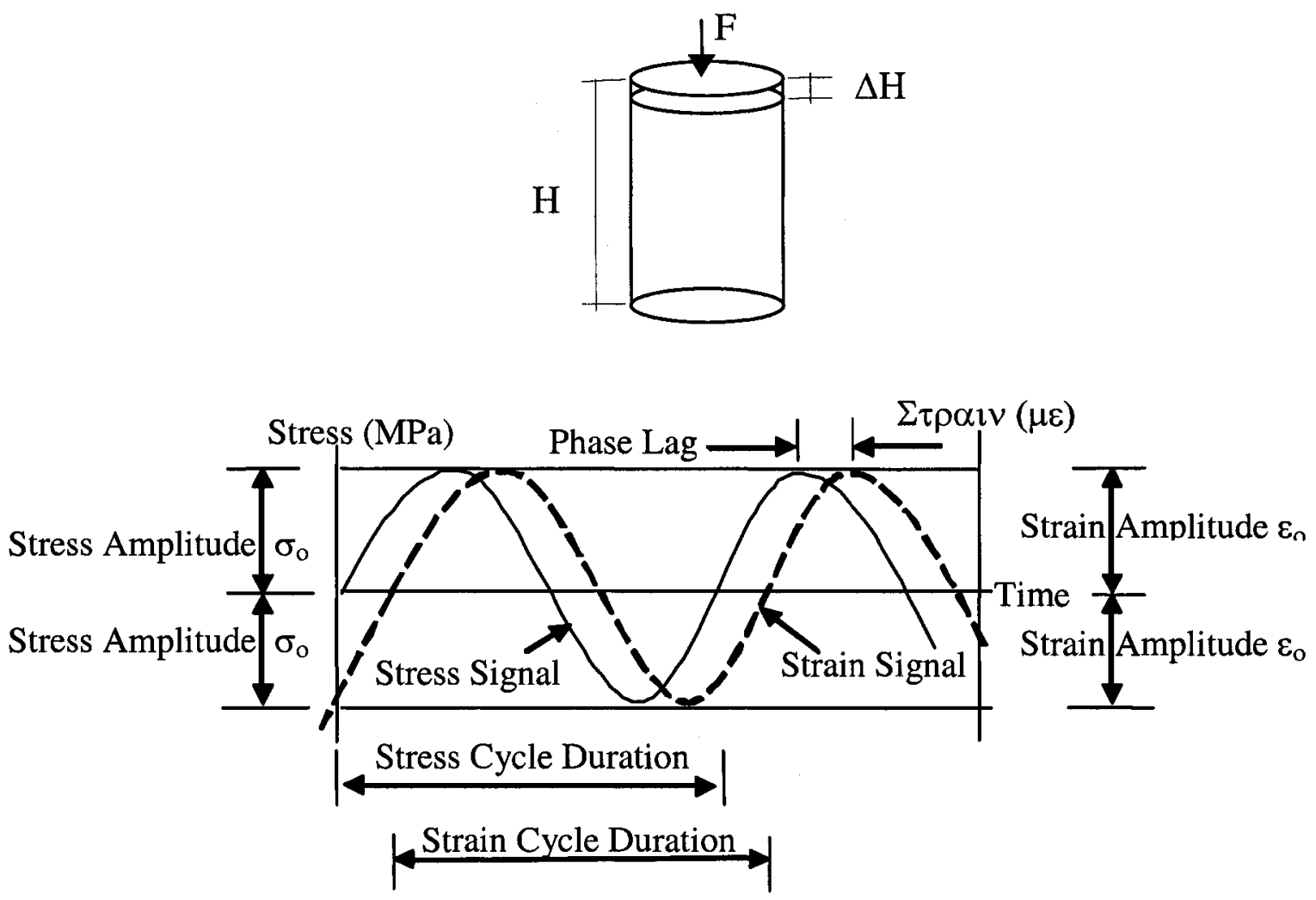

Figure 3.3. Principles of linear viscoelastic concept

To determine the linear viscoelastic properties, the material is subjected to sinusoidal loadings at different frequencies at small values of strain to conserve linearity. Loading could be performed under either a stress- or strain-controlled mode. In the first case, a specific stress value is applied and the corresponding strain is obtained, while in the other case, specific strain amplitude is applied and the corresponding stress is recorded. Equations 3.1 to 3.5 describe the viscoelastic approach mathematically [37, 40, 41, and 42].

In the stress-controlled case the stress applied is given by:

$\sigma=\sigma_{0} \operatorname{Sin}(\omega . t)$

And the corresponding strain is given by: 
$\varepsilon=\varepsilon_{0} \operatorname{Sin}(\omega . t-\phi)$

In the strain-controlled case the value of applied strain is expressed as:

$\varepsilon=\varepsilon_{0} \operatorname{Sin}(\omega . t)$

And the corresponding stress is given by:

$\sigma=\sigma_{0} \operatorname{Sin}(\omega . t+\phi)$

where $\sigma_{0}$ is the stress amplitude, $\varepsilon_{0}$ is strain amplitude (see Figure 3.1) and $\omega$ is the angular velocity related to the frequency $f$ by Equation 3.5

$\omega=2 \pi f$

$\phi$ is the phase angle related to the time that the strain lags the stress (see Figure 3.1). The phase angle is an indicator of the degree of the viscoelastic behaviour of asphalt concrete materials. The phase angle $\phi$ values are limited to between 0 and $\pi / 2$. A value of 0 is an indicator of a purely elastic behaviour, while a value of $\pi / 2$ is an indicator of a purely viscous behaviour.

However, it is useful to express the sinusoidal relations in the complex notation in which they are commonly dealt with. Hence, the previous functions can be rewritten as follows:

In the stress-controlled case the applied stress function is given by Equation 3.6:

$\sigma=\sigma_{0} \cdot e^{i w t}$

The corresponding strain is given by Equation (3.7):

$\varepsilon=\varepsilon_{0} \cdot e^{i(w t-\phi)}$

In the strain-controlled case, the function of the applied strain is expressed as: 
$\varepsilon=\varepsilon_{0} \cdot e^{i(w t)}$

And the corresponding stress is given by $\sigma=\sigma_{0} \cdot e^{i(w t+\phi)}$

\subsection{Previous Attempts at Characterizing AC Materials}

In 1962, Papazian made the first attempt to describe viscoelastic properties of asphalt concrete mixtures [46]. A sinusoidal stress was applied to a cylindrical specimen at a given frequency and the sinusoidal strain response was measured. The test was conducted under controlled temperature conditions at varying load amplitudes and frequencies. It was concluded that viscoelastic concepts could be applied in asphalt pavement design and for performance evaluation.

Research work related to the evaluation of $\mathrm{AC}$ viscoelastic response continued in the following decade. This time, different loading conditions were considered including compression, tension, and tension-compression. A number of studies indicated differences in $|E *|$ values obtained from different loading conditions. The differences affect the phase angle especially and tend to become significant at higher test temperatures. This conclusion was supported by Heck et al. [37], who reported that because of the viscoelastic behaviour and temperature sensitivity of asphalt concretes, the reversible strain signals appear to be most sensitive to time, temperature and traffic conditions. This situation made the determination of a unique elastic modulus for asphaltic materials somewhat difficult.

Witczak and Root performed complex modulus tests under five different modes of loading. However, different results were obtained for the different modes. They concluded that the tension-compression test may be more representative of field loading 
conditions [47]. This conclusion has been affirmed by Khanal and Mamlouk [48]. Bonnaure et al. [49] determined the complex modulus of asphalt mixtures from bending tests using a trapezoidal specimen fixed at one end and subjected to a sinusoidal load at the free end.

In the 1980s and early 1990s RILEM Technical Committee on Bitumen and Asphalt Testing organized an international testing program to promote and develop mix design methodologies and associated significant measuring methods for asphalt pavements [30, 50]. During this program, 15 participating laboratories throughout Europe performed complex modulus tests. Measurements were made at various temperatures and frequencies, and each laboratory used different specimen shapes, test setup, and loading conditions. The determined complex modulus and phase angle were reported and the results showed that bending tests and indirect tension tests were in reasonable agreement under certain conditions. The different laboratories were able to reproduce the phase angle $\phi$ much better than the complex modulus absolute value $|E *|$.

Research continued in the 1990s through work done by the Minnesota Department of Transportation (Mn/DOT). Complex modulus tests were performed on both tall cylindrical specimens for the tension-compression test, and short cylindrical specimens for the indirect tensile test $[36,44$, and 51$]$. Results from this study revealed that the same material tested with different setups might yield different results for the dynamic modulus and phase angle.

The most comprehensive research effort started in the mid-1990s as part of the National Cooperative Highway Research Program (NCHRP) with its two projects, Project 9-19 entitled "SuperPave Support and Performance Models Management" [52] and Project 9- 
29 entitled "Simple Performance Tester for SuperPave Mix Design" [53]. This program proposed new guidelines for what has been considered the proper specimen geometry and size, specimen preparation, testing procedure, loading pattern, and empirical modelling. A new terminology was adopted by renaming the procedure as the dynamic complex modulus test.

In addition to the above key contributions discussed, there were many other attempts made to find a material property that can reflect the mechanistic behaviour of $\mathrm{AC}$ materials. The American Society for Testing and Materials (ASTM) has reactivated the old standard test method, which was set in 1979 to be used under a new designation (ASTM D3497) [54]. The ASTM standards recommended performing the dynamic modulus test on a minimum of three laboratory-prepared specimens with a height/diameter ratio of 2:1 with a minimum diameter of 4 inches. The new standard described the minimum recommended test series of both temperature and loading frequency to consist of test temperatures in an ascending order $\left(5,25\right.$ and $\left.40{ }^{\circ} \mathrm{C}\right)$ and a haversine loading of $241 \mathrm{kPa}$ to be applied without impact at frequencies of 1,4 , and 16 $\mathrm{Hz}$ sequentially for each temperature. The recommended duration of load applications is between 30 and 45 seconds.

Pursuing the identification of a stiffness parameter that can be used to predict the field performance of asphalt concrete, the Australian Road Research Board (ARRB) recommended using the indirect tensile test for determining the elastic stiffness of asphalt using a Poisson's ratio equal to 0.4 . A specimen of $100-\mathrm{mm}$ diameter is recommended for mixes with a stone size less than or equal to $20 \mathrm{~mm}$, while a specimen of $150-\mathrm{mm}$ diameter is used for mixes with a stone size greater than $20 \mathrm{~mm}$. There is no specified 
height of specimen recommended, but a minimum height of $30 \mathrm{~mm}$ can be used, with a preference of Marshall nominal height of $63.5 \mathrm{~mm}$. According to the Australian specifications, the minimum age required of a sample prior to the test is storage overnight after preparation, and at least 4 hours in a temperature cabinet for conditioning purposes. Testing the sample at $25^{\circ} \mathrm{C}$ is mandatory; however, testing at 10 and $35^{\circ} \mathrm{C}$ is optional [55]. The indirect tensile test method has been also used by Richard et al. [56].

Kim et al. [57] performed five different tests in the laboratory using two modes of tests including indirect tensile test and uniaxial tension compression. Four tests were performed in a uniaxial mode, comprising the creep, relaxation, complex modulus and impact resonance tests. Tests were performed on specimens with a 4-inch diameter and an 8-inch height cored from 9-inch thick AC slabs prepared by a rolling wheel compaction method and stored in a temperature cabinet for a 6-hour conditioning period. Although they believed that having many frequencies would lead to a better frequencytemperature curve, they only applied one of two groups of frequencies at each temperature: 1,4 , and $16 \mathrm{~Hz}$ as specified by ASTM D3497, or 5, 10, and $20 \mathrm{~Hz}$. This approach is intended to avoid excessive damage during the test. The testing sequence starts with a high frequency and moves to lower frequencies, with a minimum of 30 seconds per each frequency. The loading time should not exceed 45 seconds. They recorded load and deformation measurements from the last five cycles with 200 points per cycle [57].

Fonseca and Witczak [31] performed a stress-controlled test on specimens prepared using the SHRP Gyratory Compactor at increasing temperatures of $-17.8,4.4,21.1,37.8$, and $54.4{ }^{\circ} \mathrm{C}$ and at a decreasing order of load frequencies: $25,10,1$, and $0.1 \mathrm{~Hz}$. This 
sequence was chosen to avoid serious damage to the specimens before proceeding to the next sequential test. The test temperature was held constant to $\pm 0.5^{\circ} \mathrm{C}$ from the designated temperature using a thermal chamber. To reach and maintain the next equilibrium temperature, the specimen was kept in the chamber for overnight. The selection of extreme temperatures was done without any basis because no specific recommendation was found in the ASTM D-3497 regarding testing at extreme testing conditions. A dynamic sinusoidal stress with a peak of $0.24 \mathrm{MPa}$ was applied for all temperatures except at $54.4^{\circ} \mathrm{C}$ where a stress of $0.07 \mathrm{MPa}$ was applied to avoid excessive deformation and thus, failure of the specimens. The dynamic loads were measured through the MTS load cell, whereas the deformations were measured through two LVDT's (Linear Variable Differential Transformer) fixed $75 \mathrm{~mm}$ apart at mid-height of the specimen.

Heck et al. [37] performed the classical French alternate flexural test on trapezoidal specimens under sinusoidal strain for the crossed frequency-temperature conditions: $[1,3$, $10,30,40 \mathrm{~Hz}] \times\left[-10,0,10,20,30,40^{\circ} \mathrm{C}\right]$. Di Benedetto [38] performed complex modulus tests in a stress-controlled mode by applying a sinusoidal loading frequency of $10 \mathrm{~Hz}$ with a maximum stress of $0.9 \mathrm{MPa}$ at a temperature of $10^{\circ} \mathrm{C}$. The actual strain amplitudes obtained were $[80,100,140$, and $180 \mu \varepsilon]$. Di Benedetti concluded that the concept of the complex modulus can be considered as a good approximation for evaluating the viscoelastic properties of $\mathrm{AC}$ materials.

Table 3.2 shows a summary of temperature series and frequency sequence that were used in the past in $\mathrm{AC}$ stiffness-related tests. 
Table 3.2. Inventory of temperatures and frequencies used in previous work

\begin{tabular}{|l|l|l|l|}
\hline Reference & Temperature $\left({ }^{\circ} \mathrm{C}\right)$ & Frequency $(\mathrm{Hz})$ & Remarks \\
\hline ASTM & 5,25 and 40 & 1,4 and 16 & $\begin{array}{l}\text { Minimum recommended } \\
\text { series }\end{array}$ \\
\hline Kim et al. & 5,25 and 40 & 5,10 and 20 & \\
\hline $\begin{array}{l}\text { Fonseca and W. } \\
\text { Witczak }\end{array}$ & $\begin{array}{l}-17.8,4.4,21.1,37.8 \\
\text { and } 54.4\end{array}$ & $\begin{array}{l}25,10,1, \text { and } \\
0.1\end{array}$ & $\begin{array}{l}\text { Temperature is allowed to } \\
\text { deviate } \pm 0.5^{\circ} \mathrm{C} \text { from target }\end{array}$ \\
\hline Heck et al. & $\begin{array}{l}-10,0,10,20,30, \text { and } \\
40 .\end{array}$ & $\begin{array}{l}1,3,10,30, \\
\text { and } 40\end{array}$ & \\
\hline $\begin{array}{l}\text { The Australian } \\
\text { Specifications }\end{array}$ & 25,10, and 35 & $\begin{array}{l}\text { Test at } 25^{\circ} \mathrm{C} \text { is mandatory, } \\
10^{\circ} \mathrm{C} \text { and } 35^{\circ} \mathrm{C} \text { are } \\
\text { optional. }\end{array}$ \\
\hline Di Benedetti & 10 & $\begin{array}{l}\text { Single temperature per } \\
\text { specimen }\end{array}$ \\
\hline
\end{tabular}

\subsection{The Complex Modulus Characterization Approach:}

The complex modulus test is one of the several laboratory procedures that have been examined above. There is general agreement among researchers about its effectiveness in evaluating the fundamental stress-strain response of asphalt concrete mixes. The modulus is a complex number, which defines the relationship between the stress and strain for a linear viscoelastic material subjected to sinusoidal loading. The real part of the complex modulus is a measure of the material elasticity and the imaginary part is a measure of the viscosity. Considering the general viscoelastic Equations 3.6 and 3.7 discussed earlier, the complex modulus can be defined (by analogy to the Young modulus of elasticity) as shown in Equation 3.10 [47]:

$$
E^{*}(i w)=\frac{\sigma}{\varepsilon}=\frac{\sigma_{0}}{\varepsilon_{0}} e^{i \phi}=E_{1}+i E_{2}
$$

The ratio of the stress to strain amplitudes defines the absolute value of the complex modulus which is known as the dynamic modulus and is expressed by Equation 3.11:

$$
\left|E^{*}\right|=\frac{\sigma_{0}}{\varepsilon_{0}}
$$


The complex modulus can be split into two parts, real and imaginary. The real part, known as the storage modulus representing the elastic part of the stored energy and is defined by Equation 3.12:

$\mathrm{E}_{1}=\left|E^{*}\right| \cos \phi$

While the imaginary part, known as the loss modulus, representing the viscous part, it is the dissipated part of the energy and is defined by Equation 3.13:

$\mathrm{E}_{2}=\left|E^{*}\right| \sin \phi$

The linear viscoelastic properties (dynamic, storage and loss moduli, and phase angle) determined using the above equations are temperature and frequency sensitive. They are usually presented in conventional forms to reflect the effect of the factors mentioned earlier. Discussed below are four common methods of presenting the complex modulus test results [43].

\subsubsection{Isothermal Curves}

Isothermal curves are obtained by plotting the dynamic modulus $\left|\mathrm{E}^{*}\right|$ as a function of reduced frequency for each temperature tested in a bi-logarithmic scale $[38,42,52]$. An example of such a presentation is given in Figure 3.4.

\subsubsection{Master Curve}

Master curves are generated based on the time-temperature superposition property of asphalt mixes. The same modulus can be obtained with different combinations of temperature and frequency. This principle allows shifting the isothermal curves 
horizontally relative to a reference temperature, thereby aligning various curves to form a single master curve.

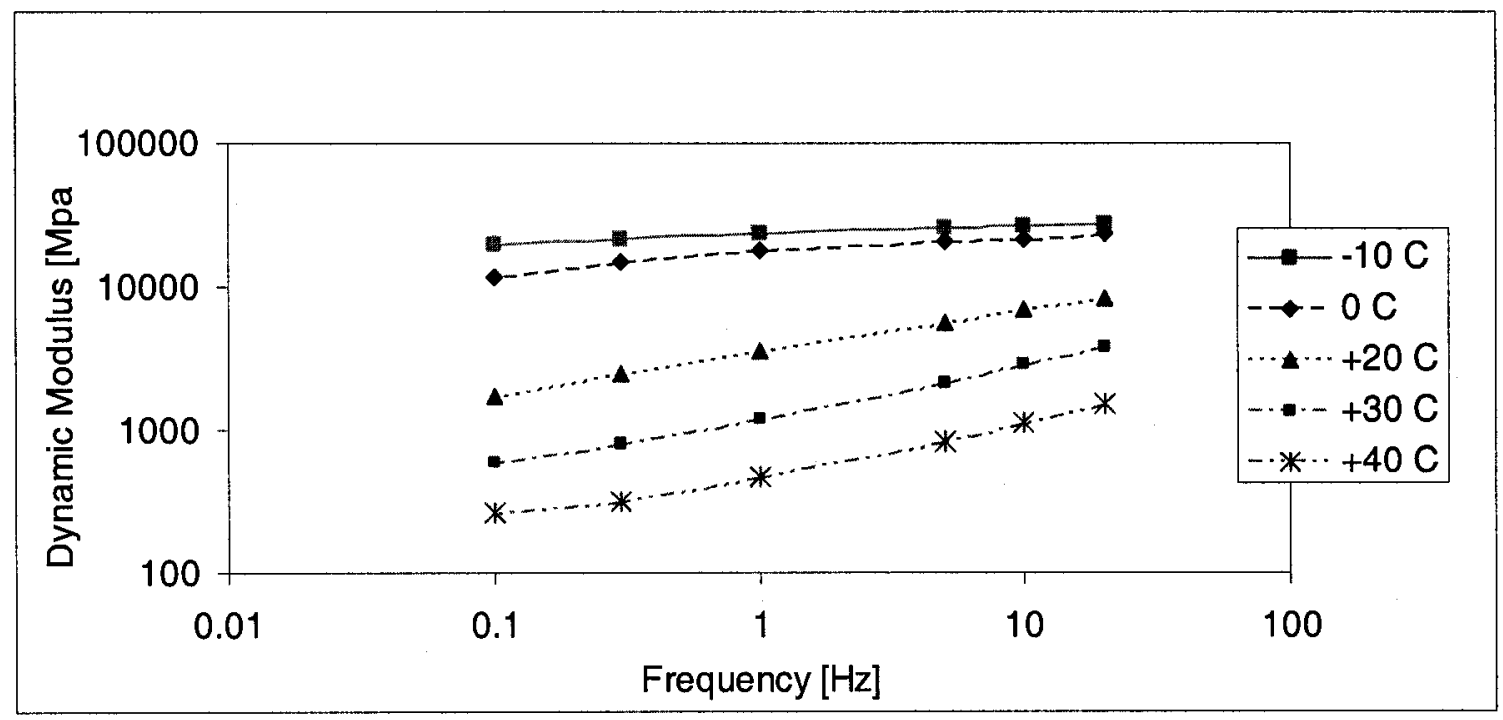

\section{Figure 3.4. Isothermal curves}

A shift factor $\alpha(T)$, defining the required amount of shift at a given temperature, is used for translating the isotherm curves to an arbitrarily selected reference temperature, $T_{R}$. Several models are used to obtain shift factors. However, the most commonly used is the William-Landel-Ferry (WLF) equation [58] as expressed in Equation 3.14:

$\log \left(\alpha_{T}\right)=\frac{-C_{1}\left(T_{i}-T_{R}\right)}{C_{2}+\left(T_{i}-T_{R}\right)}$

where $C_{1}$ and $C_{2}$ are constants that depend on the material type,

$T_{R}$ is the reference temperature, and

$\mathrm{T}_{\mathrm{i}}$ is any other testing temperature

It can be noticed that at the reference temperature, the shift factor $\alpha(T)$ is equal to one. 
The master curve gives the relationship between the dynamic complex modulus and the reduced frequency $\left[f_{r}\right]$. The reduced frequency is obtained by dividing the actual frequency by the shift factor $\alpha(T)$ as shown in Equation 3.15.

$$
f_{r}=\frac{f}{\alpha(T)} \text { Or } \log \left(f_{r}\right)=\log (f)-\log [(\alpha(T)]
$$

where $f=$ actual frequency applied in a test, $\mathrm{Hz}$

$\alpha(T)=$ shift factor by which an isotherm curve is translated horizontally to a reference temperature

Mathematically, the master curve can be represented by a non-linear sigmoidal function of the form shown in Equation 3.16 [59]:

$$
\log \left|E^{*}\right|=a+\frac{b}{1+e^{c+d\left(\log \left(t_{r}\right)\right)}}
$$

where $t_{r}$ is the time of loading at the reference temperature,

$a$ is the minimum value of $\left|E^{*}\right|$, and

$b$ represents the maximum value of $\left|E^{*}\right|$, while $c$ and $d$ are parameters describing the shape of the sigmoidal function.

The sigmoidal function of the master curve can be justified by physical observations of the AC mix behaviour. The upper part of the function approaches asymptotically the mix's maximum stiffness, which depends on the binder stiffness at cold temperatures. At high temperatures, compressive loading causes aggregate interlock stiffness to be an indicator of mixture stiffness. 
The function described above captures the physical behaviour of asphalt mixes observed in complex modulus testing throughout the entire temperature range [60]. The master curve enables expanding the dynamic modulus values beyond the actual frequencies used in the test. Figure 3.5 shows a typical master curve constructed with $0^{\circ} \mathrm{C}$ as reference temperature.

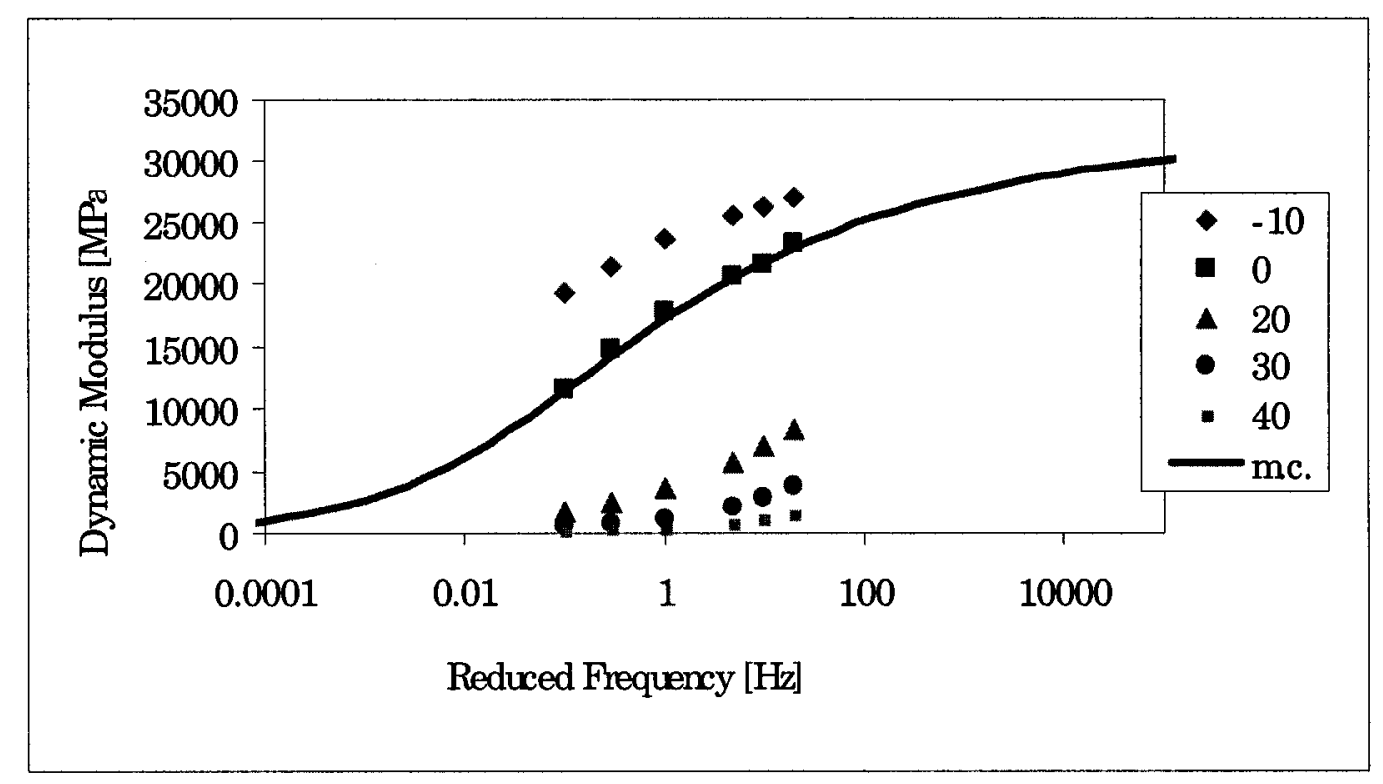

Figure 3.5. Development of a master curve

\subsubsection{Cole-Cole Diagram}

A Cole-Cole diagram is obtained by plotting the loss modulus versus the storage modulus (see Figure 3.6). Huet [42] showed that this representation could yield a unique curve that resembles an arc of a circle independent of frequency and temperature used. This representation can be used to fit a rheological model. 


\subsubsection{Black Space}

This is obtained by plotting the dynamic modulus versus the phase angle as shown in Figure 3.7. Such a representation allows a better visualization of loss modulus characterizing the behaviour of AC materials at high temperatures.

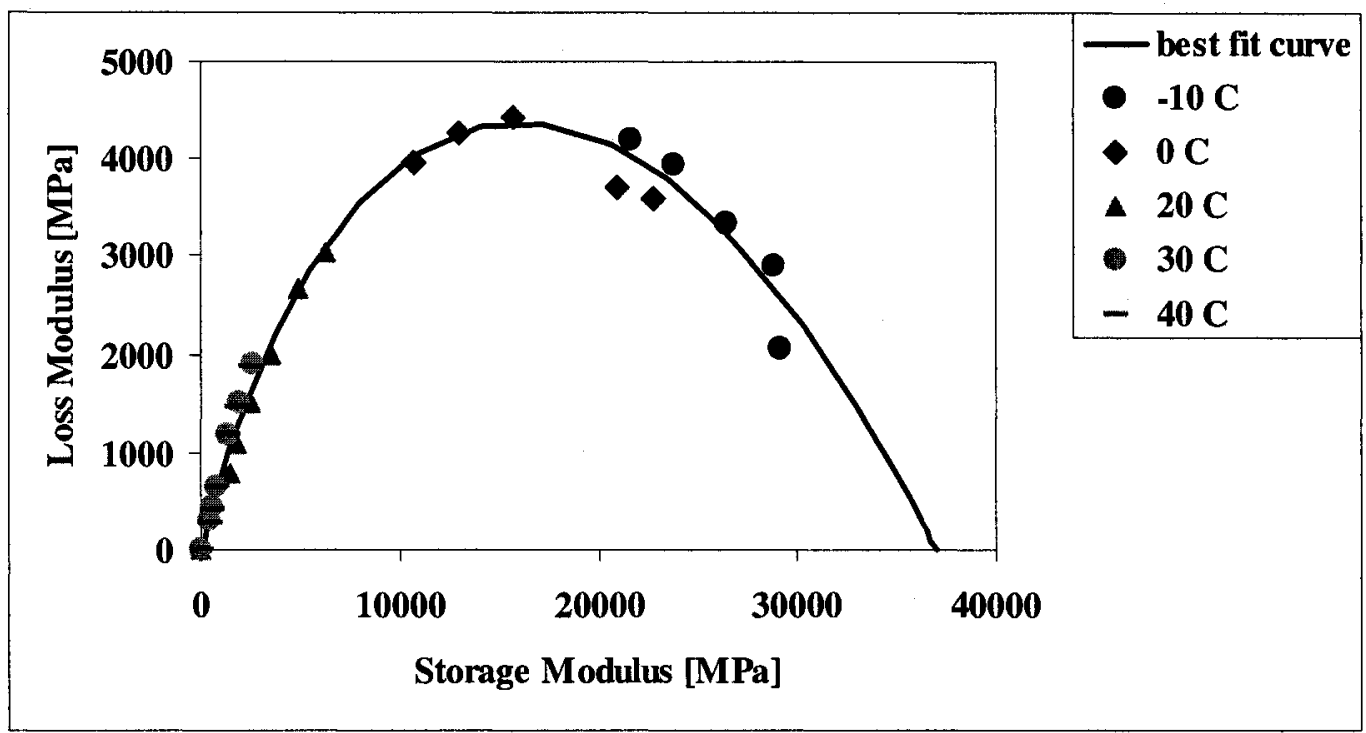

Figure 3.6. Complex modulus in Cole-Cole plane

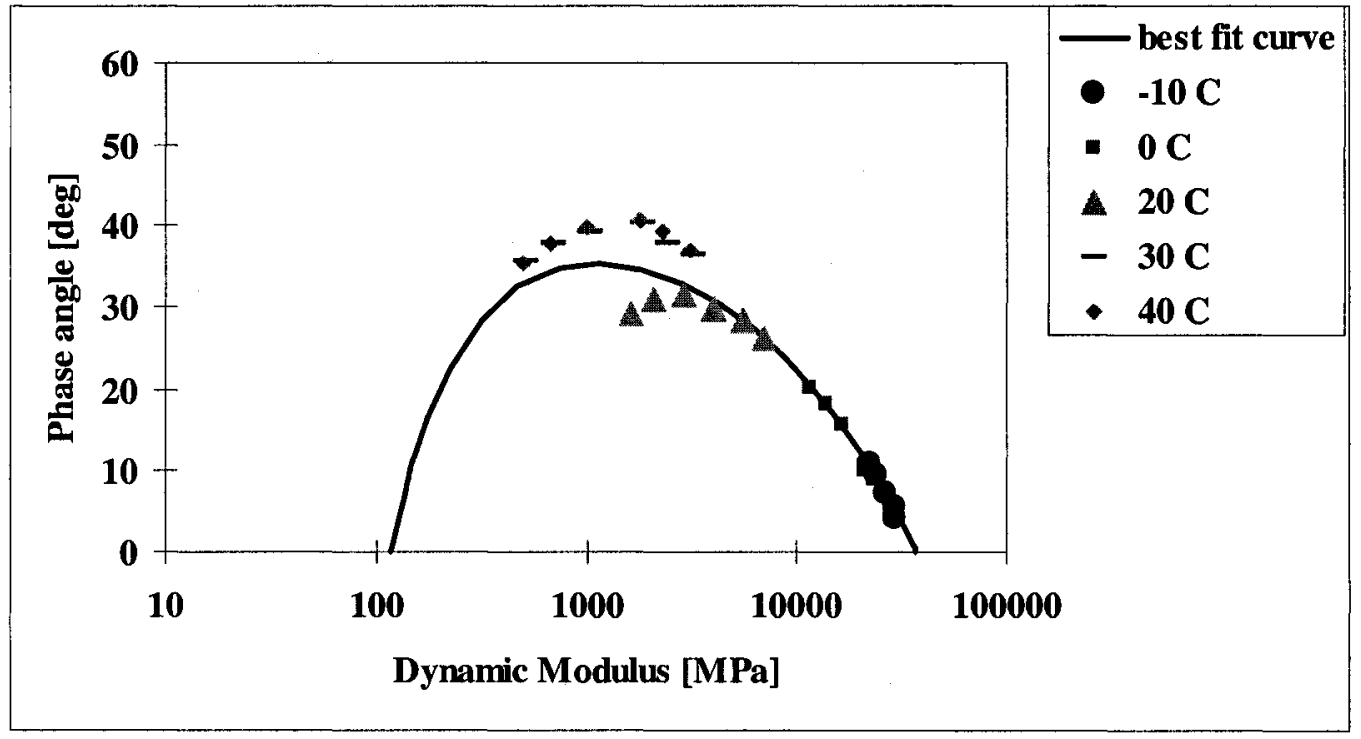

Figure 3.7. The complex modulus in Black space 


\subsubsection{Isochrone Curves}

The isochrone curves are obtained by plotting the dynamic modulus $\left|E^{*}\right|$ as a function of temperature for each frequency tested in semi-logarithmic scale [45]. Typical isochrone curves plotted using test data for a mix prepared according to Ontario Ministry of Transportation (MTO) standards, referred to as HMA 3, are shown in Figure 3.8.

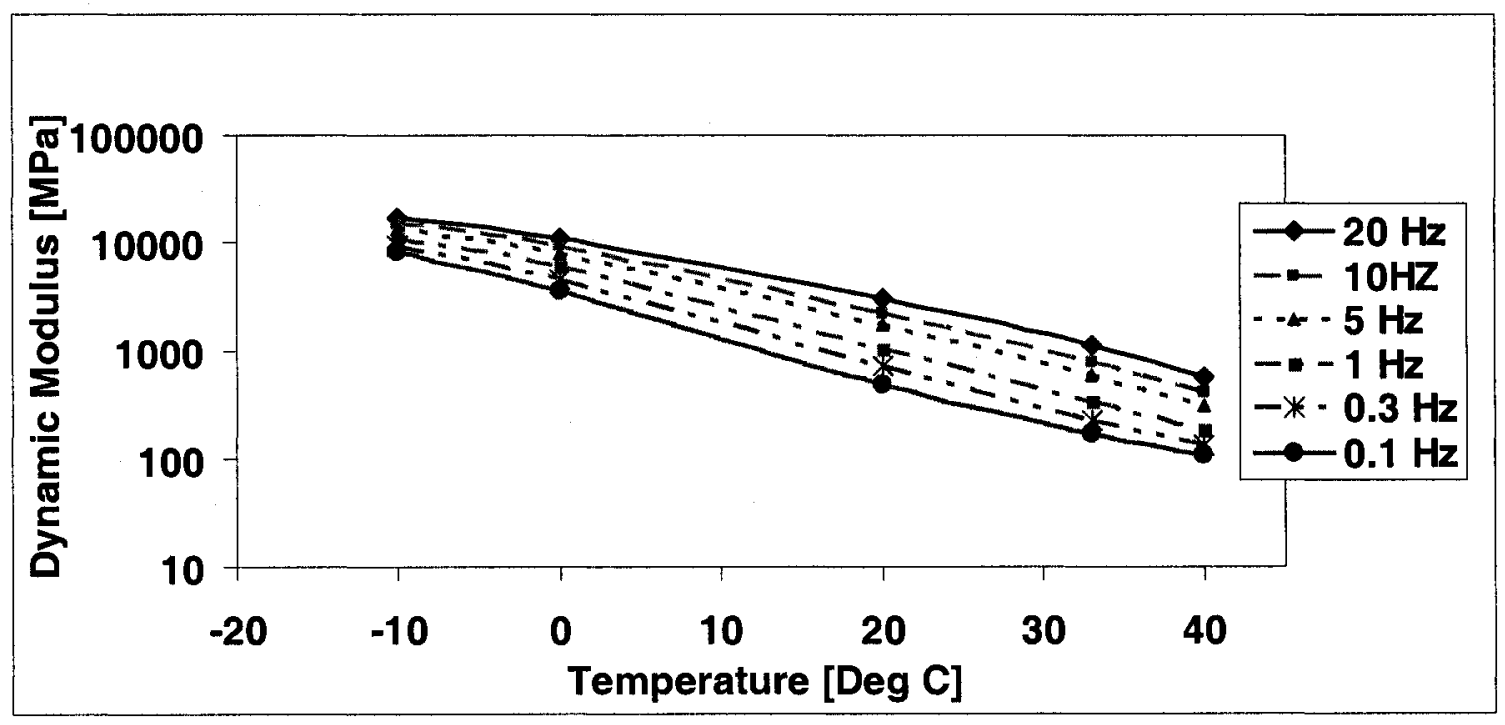

Figure 3.8. Isochrone curves of the complex modulus

\subsection{Applications of the Complex Modulus}

The complex modulus test output has several applications. For instance, the dynamic modulus is used widely in rheological models that characterize AC materials. Such rheological models could be incorporated into analytical models that can be used in the design and analysis of road pavements. In a recent application, the dynamic modulus has been incorporated as an input in the design procedure proposed into the AASHTO 2002 Design Guide discussed earlier in Chapter 2. This design guide is currently under evaluation. Also, the dynamic modulus emerged as a lead candidate for a simple performance test to predict rutting and fatigue cracking in asphalt pavements [61]. 
Moreover, complex modulus test outputs can be used to generate and validate predictive equations needed for predicting dynamic modulus values using physical properties of the asphalt mix components. This last application is intended for avoiding the need to conduct the test where physical properties are used instead to predict the complex modulus needed for running mechanistic models. The following sections highlight major applications of the complex modulus test results.

\subsubsection{Rheological Models}

Rheology, the study of a material's response to an imposed load, is a useful control tool for distinguishing between various asphalt mixes. Rheological properties are essential for effective $\mathrm{AC}$ mix design involving the selection of the key parameters for combining a mineral aggregate with the binder at the appropriate temperature to facilitate adhesion and to specify compaction requirements that may facilitate achieving an adequate composite material in the field [62]. More importantly, this response is generally used as input in performance models to predict various types of distress. The use of rheological principles to characterize the viscoelastic properties defining the performance of $\mathrm{AC}$ materials was one of the main challenges in the asphalt industry. The success of any rheological model is measured by its ability to predict stresses and strains produced by traffic and environmental loading. Since asphalts in paved roads exhibit viscoelastic behaviour under the action of moving loads, it is not surprising that the currently adopted linear elasticity approach was regarded as lacking the accuracy to predict actual pavement response and that linear viscoelasticity theory leads to a better prediction. In that sense, many rheological models such as the Generalized, Burgers, Huet and Huet-Sayegh 
models have been developed to describe the mechanistic response of bituminous mixtures. These rheological models are described in the following subsections.

\section{The Generalized Model}

It is composed of a number of different Maxwell and Kelvin elements placed in series as shown in Figure 3.9. The spring element is used to represent the elastic response, the dashpot the viscous flow and the Kelvin elements in series the delayed elastic response. The generalized model gives a good description of the viscoelastic behaviour if a sufficient number of Kelvin elements are used. However, it requires that a large number of parameters be determined [63], which has limited its use. The complex modulus of the generalized model is given by Equation 3.17 [64].

$$
E^{*}(i w)=\frac{1}{\frac{1-i\left(w \frac{\eta}{E}\right)^{-1}}{E}+\sum_{j=1}^{N} \frac{1}{E_{j}\left(1+\left(i w \frac{\eta_{j}}{E_{j}}\right)\right.}}
$$

Where $\mathrm{E}$ is the elastic response as represented by the springs, and $\eta$ is the viscous flow as expressed by the dashpots described above.

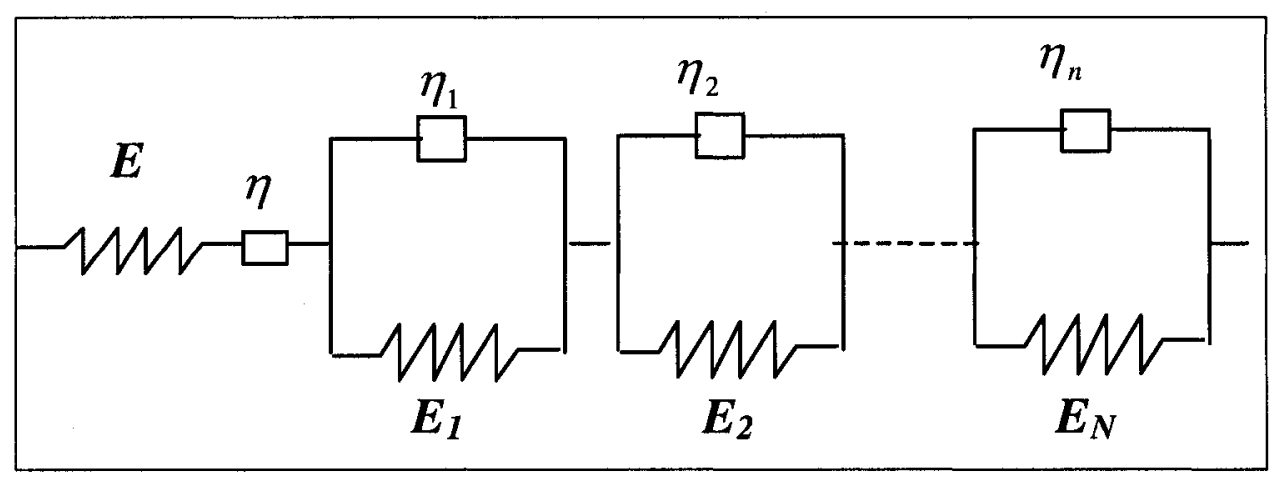

Figure 3.9. Schematic representation of the Generalized model 


\section{Burgers Model}

The Burgers model is a simplified pattern of the generalized model in which a simplification was made by reducing the number of Kelvin elements. The simplest model can be obtained by using Maxwell and Kelvin elements as shown in Figure 3.10. The Burgers model expresses the complex modulus of AC mixtures by Equation 3.18:

$$
E^{*}(i w)=\frac{E}{1+\delta\left(i w \frac{\eta}{E}\right)^{-1}}+\frac{E_{1}}{1+\delta\left(i w \frac{\eta_{1}}{E_{1}}\right)^{-1}}
$$

Though it requires the determination of only four parameters per temperature, this model has the ability to represent the viscoelastic behaviour of bituminous mixes only over a limited range of frequencies experienced by pavements under traffic and environmental loads [64].

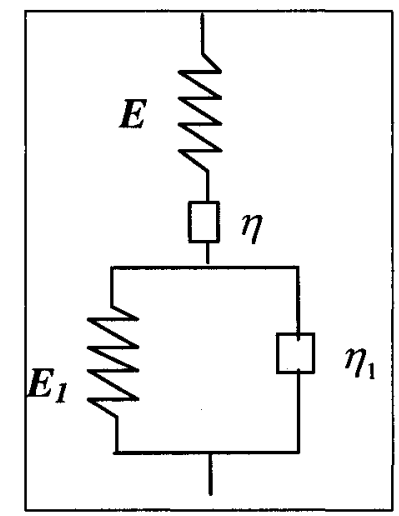

Figure 3.10. Schematic representation of Burgers model

\section{Huet Model}

Huet proposed this model in 1963 to represent the actual behaviour of AC materials. It consists of a spring $\left(\mathrm{E}_{\infty}\right)$ to represent $\mathrm{AC}$ stiffness and two parabolic dashpots (h and $\mathrm{k}$ ) placed in series as shown in Figure 3.11 to represent the viscous component of the AC 
mix response. The analytical expression of the complex modulus of the Huet model is given by Equation 3.19 [42].

$$
E^{*}(i w \tau)=\frac{E_{\infty}}{1+\delta(i w \tau)^{-k}+(i w \tau)^{-h}}
$$

The parameters $h$ and $k$ of this model are related to the tangent at the origin and the asymptotic point $\mathrm{E}_{\infty}$. Huet found that asphalt mixes behave as fluids at low frequencies and at high temperatures resembling the behaviour of the viscoplastic condition. In this model, the curve passes at the origin as shown in Figure 3.13.

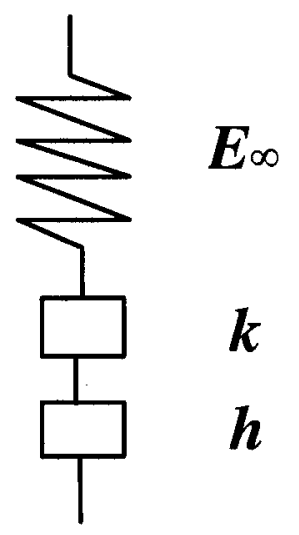

Figure 3.11. Schematic representation of the Huet model

\section{Huet-Sayegh Model:}

Contrary to Huet, in 1965 Sayegh found that at low frequencies and high temperatures the complex modulus is a non-zero real number, which is very small [42] compared to $E_{\infty}$ as shown in Figure 3.13. Accordingly, the Huet-Sayegh model was developed to comprise from two parallel branches as shown in Figure 3.12. The first branch consists of two biparabolic dashpots, $k$, and, $h$, and a spring $E_{\infty}-E_{0}$, in series. The second branch consists of a single spring, $E_{0} . E_{\infty}$ represents the purely elastic modulus of asphalt materials. On the other hand, $E_{0}$ reflects the long-term behavior of solid asphalt concrete. 
If the high temperature stiffness is set to zero, the output will be the Huet model described earlier.

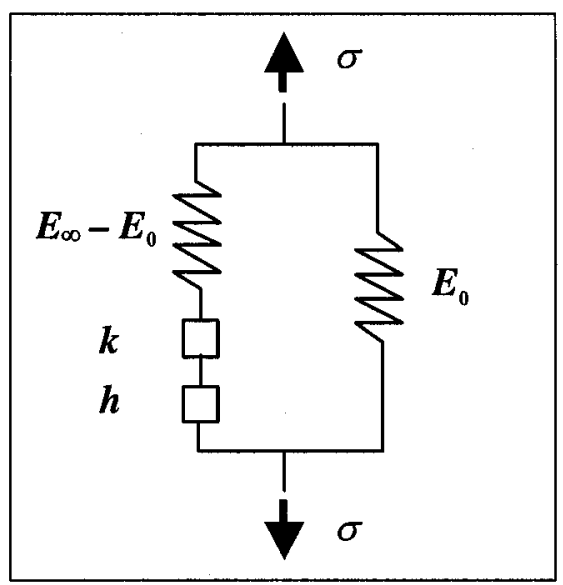

Figure 3.12. Schematic representation of the Huet-Sayegh models

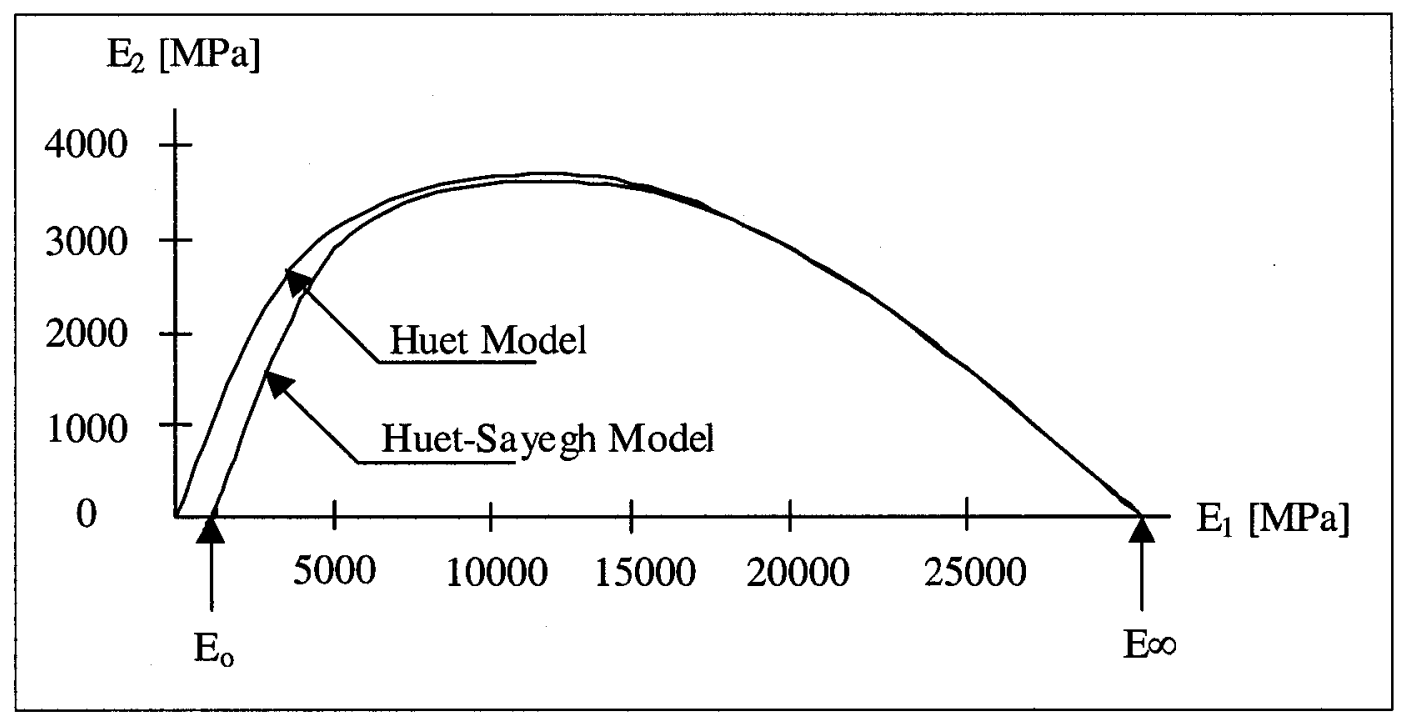

Figure 3.13. Complex modulus curves representing the Huet and Huet-Sayegh models in Cole-Cole plan

The Huet-Sayegh model was found to describe the behavior of asphalt mixes over a wide range of temperatures and frequencies and was adopted in this study. The Huet-Sayegh Model shown in Figure 3.12 is simulated analytically using the formula shown in Equation 3.20. 
$E^{*}(i \omega \tau)=E_{0}+\frac{E_{\infty}-E_{0}}{1+\delta(i \omega \tau)^{-k}+(i \omega \tau)^{-h}}$

The complex modulus $\left(\mathrm{E}^{*}\right)$ is a property that reflects the viscoelastic behaviour of $\mathrm{AC}$ material. $E_{0}$ is the high temperature stiffness and $E_{\infty}$ is the purely elastic modulus; $\delta, k$, and $h$ are parameters of the biparabolic elements of the model. The parameter, $\tau$, is referred to as the "characteristics time" and it is a temperature dependent variable calculated using Equation 3.21.

$\ln (\tau)=a+b T+c T^{2}$

where $T$ is the test temperature, and

$a, b$, and $c$ are material parameters.

Equation 3.20 can be written in a simplified form suitable for numerical implementations as follows:

$E^{*}(i \omega \tau)=E_{1}(\omega \tau)+i E_{2}(\omega \tau)$

where $E_{1}$ is called the storage modulus and can be determined by Equation (3.23) and $E_{2}$ is known as the loss modulus and can be obtained from Equation 3.24

$$
\begin{aligned}
& E_{1}=E_{0}+\frac{J_{1}}{J_{1}^{2}+J_{2}^{2}} \\
& E_{2}=\frac{J_{2}}{J_{1}^{2}+J_{2}^{2}} \ldots \ldots \ldots
\end{aligned}
$$

$J_{1}$ and $J_{2}$ are parameters expressed in terms of other parameters as shown in Equations 3.25 and 3.26 . 
$J_{1}=\frac{1+\delta \cos \left(\frac{k \pi}{2}\right)(\omega \tau)^{-k}+\cos \left(\frac{h \pi}{2}\right)(\omega \tau)^{-h}}{E_{\infty}-E_{0}}$

$$
J_{2}=\frac{\delta \sin \left(\frac{k \pi}{2}\right)(\omega \tau)^{-k}+\sin \left(\frac{h \pi}{2}\right)(\omega \tau)^{-h}}{E_{\infty}-E_{0}}
$$

\subsubsection{Design and Analysis:}

The main potential application of the dynamic modulus parameter is in the field of roadway pavement design and analysis. As mentioned previously, the AASHTO 2002 Guide for Design of Pavement Structures incorporated the dynamic modulus as a design parameter into the proposed mechanistic-empirical design procedure [60]. Level 1 analysis of the Guide requires dynamic modulus test data to develop a master curve and shift factors based on equations developed for this purpose. Level 2 Analysis constructs the master curve using actual asphalt binder test data, based on the relationship between binder viscosity and temperature. Level 3 Analysis requires no laboratory test data; instead, the software included in the 2002 Design Guide uses the Witczak model [60] to predict the dynamic modulus based on aggregate and binder properties. This last approach is discussed below in more detail.

\subsubsection{Predictive Equations}

The best way to obtain the dynamic modulus of $\mathrm{AC}$ materials is to perform a complex modulus test in the laboratory. However, given the complexity and time-consuming nature of the laboratory test procedure, many predictive equations have been proposed to evaluate the dynamic modulus of asphalt mixes using the results of simple and commonly performed aggregate and binder tests. Witczak and Fonseca [31] summarized the most 
important predictive equations developed since 1967 as they are reproduced in Table 3.3.

Table 3.3. Summary of dynamic modulus predictive equations

\begin{tabular}{|c|c|}
\hline $\begin{array}{l}\text { Equation } \\
\text { Number }\end{array}$ & Equation Model Form \\
\hline 1 & $\log _{10}\left|E^{*}\right|=a_{0}+a_{1} p_{200}+a_{2} V_{a}+a_{3} \eta_{70: 10^{6}}+a_{4} p_{a c}{ }^{a} 5 t_{p}{ }^{a} 6$ \\
\hline 2 & $\log _{10}\left|E^{*}\right|=b_{0}+b_{1} p_{200}+b_{2} V_{a}+b_{3} p_{a c}^{b} 4\left(\log \eta_{t}\right)^{b} 5$ \\
\hline 3 & $\log _{10}\left|E^{*}\right|=c_{0}^{*} c_{1} p^{-\left(t c_{2}\right)}$ \\
\hline 4 & $\begin{array}{l}\log _{10}\left|E^{*}\right|=d_{0}+d_{1}\left(p_{200}\right) f^{d_{2}}+d_{3} V_{a}+d_{1} \eta_{70: 10^{6}}+ \\
d_{5}\left[t_{p}^{\left(d_{6}+d_{7} \log f\right)} p_{a c}^{d_{8}}\right]+d_{9}\left[t_{p}^{\left(d_{6}+d_{7} \log f\right)} p_{a c}^{d_{8}} f^{d_{10}}\right]+d_{11} f^{d_{12}}\end{array}$ \\
\hline 5 & $\log _{10}\left|E^{*}\right|=e_{0}+e_{1} V_{a}+e_{2} \eta_{70: 10^{6}}+e_{3} t_{p}^{e_{4}} V_{b e f f}^{e_{5}}$ \\
\hline 6 & $\begin{array}{l}\log _{10}\left|E^{*}\right|=g_{0}+g_{1}\left(p_{200}\right) f^{g_{2}}+g_{3} V_{a}+g_{4} \eta_{70: 10^{6}}+g_{5} f^{g_{6}} \\
\left.+g_{7} t_{p}^{\left(g_{8}+g_{9} \log f\right)}+g_{10} f^{g_{11}} t_{p}^{\left(g_{8}+g_{9} \log f\right)}\right]\left[p_{a c}-p_{o p t}+g_{12}\right]^{g_{13}}\end{array}$ \\
\hline 7 & $\begin{array}{l}\log _{10}\left|E^{*}\right|=h_{0}+h_{1} V_{a}+h_{2} p_{3 / 4}+h_{3} \eta_{70: 10^{6}}+h_{4} t_{p}+h_{5} \log f+ \\
h_{6}\left(\log f^{*} t_{p}^{2}\right)+h_{7}\left(V_{\text {beff }}-V_{\text {beffopt }}+h_{8}\right)^{h_{9}} t_{p}{ }^{2}+h_{10}\left(V_{\text {beff }} p_{4}\right)+h_{11}\left(p_{200} p_{a b s}\right)\end{array}$ \\
\hline 8 & $\begin{array}{l}\log _{10}\left|E^{*}\right|=k_{0}+k_{1} V_{b e f f}+k_{2} V_{a}+k_{3} p_{200}+k_{4} p_{4}+k_{5} p_{a b s}+k_{6} t_{p}+k_{7} f+k_{8} t_{p}^{2}+k_{9} V_{b e f f}{ }^{2}+ \\
k_{10} p_{200}{ }^{2}+k_{11} p_{3 / 4}^{2}+k_{12} p_{3 / 8}^{2}+k_{13} p_{4}^{2}+k_{14} p_{a b s}{ }^{2}+k_{15} \eta_{70: 10^{6}}+k_{16} f^{2}+k_{17} p_{3 / 8} V_{b e f f}+ \\
k_{18} p_{3 / 8} V_{b e f f}+k_{19} p_{3 / 4} p_{4}+k_{20} p_{3 / 8} p_{4}+k_{21} p_{3 / 8} p_{a b s}\end{array}$ \\
\hline 9 & $\begin{array}{l}\log _{10}\left|E^{*}\right|=l_{0}+l_{1} V_{b e f f}+l_{2} V_{a}+l_{3} p_{200}+l_{4} p_{a b s}+l_{5} t_{p}+l_{6} f+l_{7} t_{p}^{2}+ \\
l_{8} V_{b e f f}^{2}+l_{9} p_{200}^{2}+l_{10} p_{3 / 4}^{2}+l_{11} p_{3 / 8}^{2}+l_{12} p_{a b s}{ }^{2}+l_{13}\left(\eta_{70: 10^{6}}\right)^{2}+l_{14} f^{2}+ \\
l_{15} p_{3 / 8} V_{b e f f}+l_{16} p_{3 / 4} V_{b e f f}+l_{17} p_{3 / 4} p_{4}+l_{18} p_{3 / 8} p_{4}+l_{19} p_{3 / 8} p_{a b s}\end{array}$ \\
\hline 10 & $\begin{array}{l}\log _{10}\left|E^{*}\right|=m_{0}+m_{1} V_{\text {beff }}+m_{2} V_{a}+m_{3}\left(\eta_{70: 10^{6}}\right)+m_{4} t_{p}+m_{5} \log f+ \\
m_{6} \log \left(f^{*} t_{p}{ }^{2}\right)+m_{7}\left(V_{\text {beff }}-V_{\text {beffopt }}+m_{8}\right)^{m_{9}} t_{p}^{2}+m_{10}\left(V_{\text {beff }} p_{4}\right)+m_{11}\left(p_{200} p_{a b s}\right)\end{array}$ \\
\hline
\end{tabular}

In the equations shown in Table 3.3, the alphabetic letters subscripted with numbers are regression coefficients. The other variables have the following definitions and units:

$\eta_{70: 10^{6}}=\mathrm{AC}$ viscosity at $70^{\circ} \mathrm{F}\left(21.1^{\circ} \mathrm{C}\right)$, in $10^{6}$ poise

$\eta_{t}=\mathrm{AC}$ viscosity at test temperature $(\mathrm{t})$, in poise 
$t_{p}=$ Test temperature, in ${ }^{\circ} \mathrm{F}$

$f=$ Test frequency of load wave, in $\mathrm{Hz}$

$V_{a}=$ Percent volume of air voids in the mix

$V_{b e f f}=$ Effective asphalt content of mix, by volume percentage

$V_{\text {beffopt }}=$ Effective optimum asphalt content of mix, by volume percentage

$p_{a b s}=$ Percentage asphalt absorption

$p_{a c}=$ Percentage asphalt content, by weight of mix

$p_{\text {opt }}=$ Optimum asphalt content of mix, in percent by weight of mix

$p_{3 / 4}=$ Percent weight (by total aggregate weight) retained on $3 / 4$ inch sieve

$p_{3 / 8}=$ Percent weight (by total aggregate weight) retained on $3 / 8$ inch sieve

$p_{4}=$ Percent weight (by total aggregate weight) retained on a No. 4 sieve

$p_{200}=$ Percent weight (by total aggregate weight) passing through a No. 200 sieve

However, the models presented in Table 3.3 have several limitations as reported by Witczak [31]. The major drawback of these equations is associated with classical statistical principles regarding model extrapolations of parameters outside the range of variables used to develop the model. In addition, all the test data were generated within the temperature range of 5 to $40^{\circ} \mathrm{C}$. This resulted in unrealistically large and small predictive moduli for very cold and very hot conditions outside this range. 
Also Fonseca and Witczak [31] observed that the majority of predictive equations developed so far are based on the original bitumen properties, with the test temperature being the most important variable in the system. However, these earlier predictive equations do not account for the hardening effects on binder, and consequently the $\mathrm{AC}$ properties associated with long-term aging.

Witczak proposed two predictive equations for the complex modulus based on a large amount of data consisting of 1429 points from 149 separate asphalt concrete mixes. Improvements were made to earlier models taking into account hardening effects from short and long-term aging, as well as extreme temperature conditions. Based on the gradation of aggregates in the mix and asphalt binder properties, the first Witczak dynamic modulus model was published in 1996 from this statistical study as shown in Equation 3.27 [31].

$$
\begin{aligned}
& \log \left|E^{*}\right|=-0.261+0.008225 P_{200}-0.00000101\left(P_{200}\right)^{2}+0.00196 P_{4}-0.03157 V_{a} \\
& -0.415 \frac{V_{\text {beff }}}{\left(V_{\text {beff }}+V_{a}\right)}+\frac{\left[1.87+0.002808 P_{4}+0.00000404 P_{38}-0.0001786\left(P_{38}\right)^{2}+0.0164 P_{34}\right]}{1+e^{(-0.716 \log f-0.7425 \log \eta)}}
\end{aligned}
$$

where:

$\left|E^{*}\right|=$ Asphalt mix dynamic modulus, in $10^{5} \mathrm{psi}$,

$\eta=$ Bitumen viscosity, in $10^{6}$ poise,

$f=$ Loading frequency, in $\mathrm{Hz}$,

$\mathrm{V}_{\mathrm{a}}=$ Percent air voids in the mix, by volume,

$V_{\text {beff }}=$ Percent effective bitumen content, by volume, 
$P_{34}=$ Percent retained on $3 / 4$-inch sieve, by total aggregate weight (cumulative),

$P_{38}=$ Percent retained on 3/8-inch sieve, by total aggregate weight (cumulative),

$\mathrm{P}_{4}=$ Percent retained on No. 4 sieve, by total aggregate weight (cumulative), and

$\mathrm{P}_{200}=$ Percent passing No. 200 sieve, by total aggregate weight.

However, after more data had been introduced, Witczak modified the previous predictive equation for calculating the dynamic modulus, which appeared in 2000 as shown in Equation $3.28[31]$ :

$$
\begin{aligned}
& \log \left|E^{*}\right|=-1.249937+0.029232 P_{200}-0.001767\left(P_{200}\right)^{2}+0.002841 P_{4}-0.058097 V_{a} \\
& -0.802208 \frac{V_{b e f f}}{\left(V_{b e f f}+V_{a}\right)}+\frac{\left[3.871977-0.0021 P_{4}+0.00395 P_{38}-0.000017\left(P_{38}\right)^{2}+0.00547 P_{34}\right]}{1+e^{(-0.603313-0.313351 \log f-0.393532 \log \eta)}}
\end{aligned}
$$

There was no change noted in the form of the 2000 equation compared with that published in 1996. However, the constant coefficients were changed to reflect calibration achieved with more data.

\subsubsection{Performance Rating:}

The National Corporation of Highway Research Program (NCHRP) launched Project 919 with the objective of developing a Simple Performance Test (SPT) for asphalt mixes [53]. Various testing configurations were evaluated. The SPT methods were categorized as stiffness-related tests, deformability tests, and cracking tests. The stiffness parameters were obtained via three methods, namely the compressive complex modulus, the Simple Shear Tester (SST), and ultrasonic wave propagation. Of these three methods, the complex modulus appeared to be the most promising for relating material properties 
(stiffness) to rutting and fatigue cracking observed in the field. However, the study revealed that the complex modulus of asphalt mixes is not a good performance indicator for thermal cracking.

The complex modulus test showed good correlation to permanent deformation (rutting) of asphalt mixtures. Witczak et al. [65] performed research on asphalt mixtures attempting to correlate stiffness to rutting similar to that performed for fatigue cracking. The results of the study confirmed that the complex modulus test output provides a good indication of the susceptibility of $\mathrm{AC}$ mixes to rutting. 


\section{CHAPTER 4 \\ DESIGN OF THE EXPERIMENTAL INVESTIGATION}

\subsection{Introduction}

This chapter discusses components of the experimental investigation designed in response to the objectives set for this thesis including:

1) Development of a complex modulus testing procedure;

2) Establishment of an asphalt concrete (AC) characterization technique; and

3) Examination of the applicability of the proposed characterization technique in pavement design and analysis.

Elements included in these three main categories of the designed experimental investigation are outlined schematically in Figure 4.1 and discussed in detail in the following subsections.

\subsection{Development of a Complex Modulus Test Protocol}

The laboratory testing scheme was conceptualized taking into consideration the state of asphalt concrete used in road pavements and its exposure to elements of the environment and traffic loading. The testing program was developed benefiting from the previous experience of other researchers as discussed in Chapter 3. 


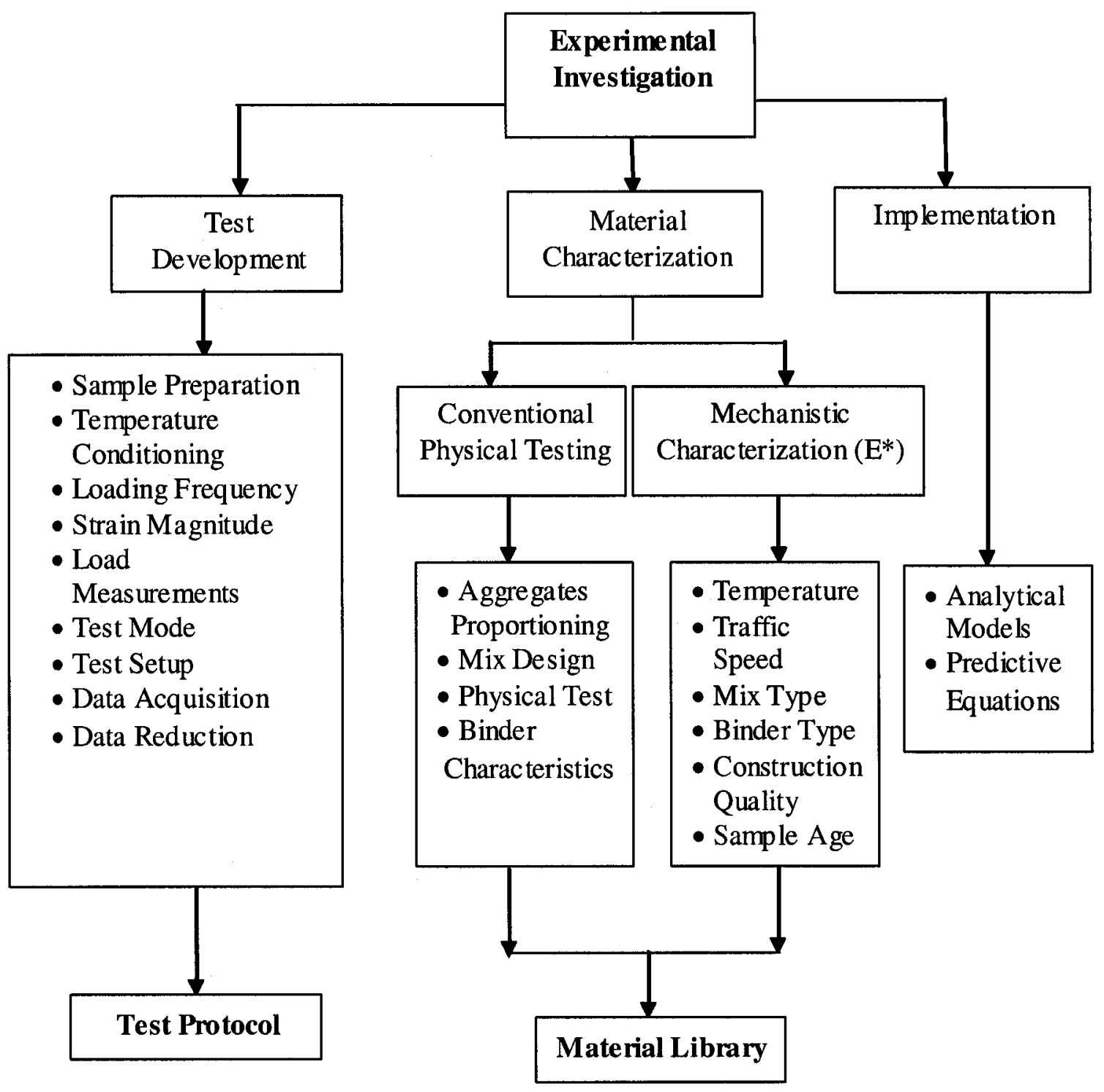

Figure 4.1. Design of the experimental investigation

The design of the test protocol involved examining factors that influence the complex modulus parameter including:

The condition of the sample, which necessitates following a standard preparation procedure;

The sensitivity of $\mathrm{AC}$ materials to temperature requires controlling the temperature of the sample during the test; 
The response of the material is influenced by load characteristics. These characteristics influence displacement magnitude that determines if there will be an elastic response or if the sample will experience permanent deformation;

The intention to simulate important traffic characteristics dictates controlling the loading frequency to account for different traffic speeds;

Equipment assembled to perform the test and the test setup should facilitate the necessary control of the above parameters and collection of the data necessary for capturing components of the material response;

The data acquisition system must be designed to record the test history involving critical sampling rates capable of recording changes in the stress and strain condition.

Elements constituting the development of the complex modulus test protocol are discussed in detail in the following subsections. Results of tests performed in the development of the test procedure will be discussed in Chapter 5.

\subsubsection{Sample Preparation}

The preparation of the composite material followed two commonly used AC mix design techniques. The conventional Marshall mix design procedure was followed to prepare three mix types for complex modulus testing. Two additional new mix types were designed to represent the SuperPave technology [18] developed during the Strategic Highway Research Program (SHRP).

The Marshall and SuperPave mixes were designed according to AASHTO Designation MP2 [66] with different binder types leading to slightly different binder contents. Standard compaction procedures were followed to prepare samples for mix design 
purposes to achieve the required physical properties included in the standard procedure based on selecting the optimum binder content. The Marshall Hammer was used to compact specimens prepared using the Marshall mix design procedure. The gyratory compactor was used to compact specimens prepared according to the SuperPave mix design procedure. The optimum binder content was selected following local specifications of the Ministry of Transportation of Ontario [71]. These binder contents were then used to prepare the AC specimens for the complex modulus test.

A mechanical mixer was used to prepare a homogenous aggregate-binder mixture. The mixture was aged in an oven for two hours at the compaction temperature of binders in accordance with the short-term aging procedure specified by AASHTO PP2. The compaction temperatures of different binders used in this investigation were specified by the binder manufacturer. All mixtures were then compacted with the SHRP gyratory compactor using a specific number of gyrations to prepare specimens for the complex modulus tests. The number of gyrations was adjusted to produce samples with the desired size at the locally specified air voids percent. The gyratory compactor was selected because of its ability to produce samples at a specified height and air voids content. This choice was also influenced by the fact that the gyratory compactor is known to produce a compacted specimen that simulates field conditions better than the specimen prepared with the Marshall hammer.

The size of specimens prepared for the complex modulus tests was determined according to ASTM specifications D3497 [67]. Samples with a diameter of $100 \mathrm{~mm}$ and a thickness of $100 \mathrm{~mm}$ were prepared. 
The number of replicates required for the test specimens was determined based on variations observed in the measured physical properties of the compacted specimens. Three specimens, with relatively similar physical properties, were prepared from each mix. Two specimens were used in the complex modulus investigation with one sample stored as a backup in case a sample was lost because of premature failure during the test (delaminating or failure due to eccentricity).

Physical properties of the compacted specimens such as bulk specific gravity, maximum specific gravity, and air voids were determined according to AASHTO specifications T 166 [68], T 209 [69] and T 269 [70] respectively. Complex modulus test specimens were prepared targeting air voids of $5 \%$ with a tolerance of $+/-0.5 \%$. The determined physical properties of the compacted specimens prepared for characterizing the mixes selected in this study will be discussed later.

The ends of the prepared cylindrical specimen were made smooth by sawing to make sure that the ends were perpendicular to the axis of the specimen. Sawing also provides a stable surface for gluing samples to the steel caps used to attach the sample to the loading rod. Completed specimens were wrapped in polyethylene and placed in an environmentally protected storage area at temperatures between 5 and $25^{\circ} \mathrm{C}$. The effect of curing on test results was considered in this study. Conditioning in the oven, which is part of the mix design standard, was maintained. However, a delay in testing a sample after its manufacturing was also organized in order to make sure that tests were performed after a relatively constant stage period. This condition was achieved by storing the replicated specimens for the same period prior to testing with a tolerance of $+/-1$ day. 
Unlike many other materials, an AC specimen cannot be gripped during the test. Instead, gluing its ends to metallic caps has been the best way to keep it from moving during load applications involving tension and compression. A special gluing apparatus was designed for gluing purposes as shown in Figure 4.2. Pelco LEP 502 epoxy provided adequate adhesion between the metallic caps and the sawed sample for the load levels experienced in this investigation. Specimens were glued to the metallic caps using the epoxy, making sure that each specimen was well centered on its axis to avoid eccentricity, which affects the test results. The glue was left to dry for minimum of six hours prior to testing.

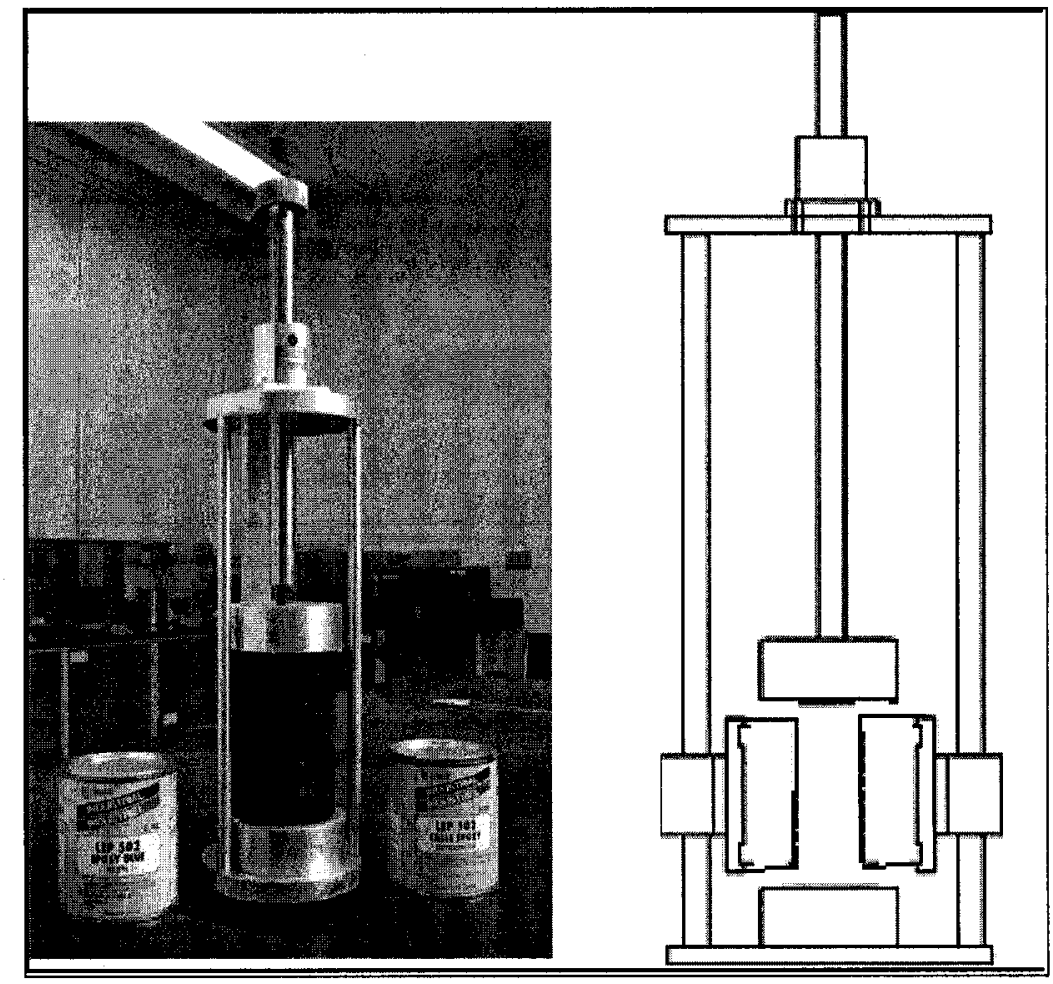

Figure 4.2. a) System used to glue samples, b) handling system details

\subsubsection{Temperature Conditioning}

As mentioned in Chapter 1, AC is used as a surface layer in more than $85 \%$ of the Canadian roadway network. However, there are temperature differences associated with seasonal variations within one location and differences within the different geographical 
regions. In Ottawa, Ontario, pavement temperatures drop as low as $-45^{\circ} \mathrm{C}$ during winter nights and rise to $+35^{\circ} \mathrm{C}$ on summer days.

Initial experimental work was conducted on an HMA 2 mix, using binder type PG 58-22 to determine applicable temperature limits that could be used for the complex modulus testing of AC materials. Attempts were made to extend testing temperatures on both cold and warm sides. Such an attempt involved using $-20^{\circ} \mathrm{C}$ to simulate low temperature conditions in cold regions. However, difficulties were encountered while attempting to perform the test at this very low temperature. Initial load repetitions needed to compensate for the material resistance to reach the controlled strain magnitude was enormous (2800 cycles), which proved to be excessive, resulting in fatigue failure. Accordingly, the $-20^{\circ} \mathrm{C}$ test temperature was eliminated from the test protocol. Test trials performed at $-10^{\circ} \mathrm{C}$ were successful; accordingly, testing at cold temperatures was limited to $-10^{\circ} \mathrm{C}$.

On the other side of the temperature range, initial attempts were made to perform the complex modulus test at temperatures as high as $+50^{\circ} \mathrm{C}$. However, non-linearity of the response reflected in the accumulation of permanent deformations resulted in the elimination of this test temperature from the testing program. Results related to this attempt performed on the SuperPave mix (SP 1), prepared with a PG 52-34 binder, are discussed in Chapter 5.

The phase angle measured at different temperatures was also used as an indicator of the linearity of the measured response. Tests were performed using a SuperPave mix (SP 1), prepared with a PG 52-34 binder. 
Limiting AC complex modulus measurements within the linear viscoelastic state in this thesis led to the selection of temperatures ranging from -10 to $+40^{\circ} \mathrm{C}$. Tests were performed in an increasing sequence in order to minimize serious damage that may occur in the specimen if tested at high temperatures before proceeding to testing at other temperatures. The complex modulus of $\mathrm{AC}$ mixes at temperatures outside the selected range could be extrapolated benefiting from the characteristics of master curves as will be discussed in Chapter 6.

For proper simulation of temperature variations in the laboratory, an environmental chamber capable of controlling the temperature over the desired range and with $+/-0.5^{\circ} \mathrm{C}$ accuracy was used for sample conditioning. The chamber selected was large enough to accommodate the test specimen, the measuring devices, and other test accessories. For high temperatures (i.e., $20^{\circ} \mathrm{C}$ and above), samples were conditioned by mechanical heating. Liquid nitrogen was used to bring the temperatures down for conditioning the sample at levels below ambient room temperature. To avoid the effect of thermal shock, a gradual transition from one temperature to another was applied. The period required for conditioning was determined by performing a test on a number of specimens with a thermocouple fixed inside.

\subsubsection{Loading Frequency:}

Vehicles are operated at various speeds on roads. To develop a test protocol sensitive to this variable, traffic speeds were represented by different loading frequencies. Frequency definition was utilized to correlate traffic speed to frequency. Frequency is defined as the number of load repetitions per second. In other words, the time duration needed to 
complete one cycle. This definition was used along with the distance-speed relationship as shown in Equations 4.1 to 4.4 :

$f=1 / \mathrm{T}$

$\mathrm{D}=\mathrm{V} * \mathrm{~T}$

then $\mathrm{V} / \mathrm{D}=1 / \mathrm{T}$

From Equations 4.1 and 4.3 the frequency can be derived as a function of traffic speed as shown in Equation 4.4:

$f=\mathrm{V} / \mathrm{D}$ 4.4

where: $f=$ Angular velocity, $\mathrm{Hz}$

$\mathrm{V}=$ Vehicular speed, $\mathrm{m} / \mathrm{s}$

$\mathrm{D}=$ Axle spacing (wheelbase), $\mathrm{m}$, and

$\mathrm{T}=$ Time required to pass the distance $\mathrm{D}, \mathrm{s}$

The wheelbase is determined as the critical axle spacing of a truck based on the conventional truck matrix reported by NCHRP [71] as shown in Table 4.1.

From Table 4.1, the maximum wheelbase of a truck is 38 feet $(11.58 \mathrm{~m})$. By substituting this value for $\mathrm{D}$ in Equation 4.4, the vehicle speeds corresponding to the selected frequencies were calculated as shown in Table 4.2. It is believed that this selection covers a wide range of vehicular speeds. The selection of vehicle speed was extended beyond normal road speeds to support analysis based on graphical representation of the test data.

Similar to the temperature situation where extending the test temperature range above $+40^{\circ} \mathrm{C}$ and below $-10^{\circ} \mathrm{C}$, which was not achieved based on the difficulties reported in section 4.2.2; attempts were made to include frequencies greater than $20 \mathrm{~Hz}$, such as 30 
and $40 \mathrm{~Hz}$. However, applying such higher frequencies was not a safe task as the testing system vibrated vigorously during the initial stage while attempting to achieve the targeted strain amplitude. On the other hand, at low frequencies, simulating a traffic speed as low as $4 \mathrm{~km} / \mathrm{h}$ (test frequency less than $0.1 \mathrm{~Hz}$ ) resulted in a situation that may be considered a static load and did not produce the targeted viscoelastic response. Results of tests performed at six different frequencies using an HMA 3 mix with a PG 64-34 binder are discussed in Chapter 5.

Table 4.1. Truck matrix with different sizes and weights

\begin{tabular}{|l|l|l|l|}
\hline Truck Number & Configuration Name & Wheelbase (ft) & Wheelbase (m) \\
\hline $1-2$ & 2 Axle Straight Truck & 15 & 4.57 \\
\hline $3-4$ & 3 Axle Straight Truck & 18 & 5.49 \\
\hline $5-8$ & 3 Axle Refuse Hauler & 17.5 & 5.33 \\
\hline $9-12$ & 4 Axle Concrete Mixer & $20 / 12$ & $6.10 / 3.66$ \\
\hline 13 & 3 Axle Tractor Semitrailer & $10 / 36$ & $3.05 / 10.97$ \\
\hline $14-15$ & 4 Axle Tractor Semitrailer & $12 / 36$ & $3.66 / 10.97$ \\
\hline $16-20$ & 5 Axle Tractor Semitrailer & $12 / 36$ & $3.66 / 10.97$ \\
\hline 21 & 5 Axle Tractor Semitrailer & $10 / 36$ & $3.05 / 10.97$ \\
\hline 22 & 5 Axle Tanker & $12 / 36$ & $3.66 / 10.97$ \\
\hline $23-24$ & 6 Axle Tanker & $12 / 38$ & $3.66 / 11.58$ \\
\hline 25 & 5 Axle Doubles & $10 / 22 / 22$ & $3.05 / 6.71 / 6.71$ \\
\hline 26 & 5 Axle Doubles & $10 / 22 / 22$ & $3.05 / 6.71 / 6.71$ \\
\hline 27 & 7 Axle Doubles & $12 / 38 / 22$ & $3.66 / 11.58 / 6.71$ \\
\hline 28 & 9 Axle Doubles & $12 / 38 / 38$ & $3.66 / 11.58 / 11.58$ \\
\hline 29 & Turner Doubles & $12 / 22 / 22$ & $3.66 / 6.71 / 6.71$ \\
\hline
\end{tabular}

Table 4.2. Vehicle speeds and corresponding frequencies

\begin{tabular}{|c|c|}
\hline Frequency $(\mathrm{Hz})$ & Vehicle Speed $(\mathrm{km} / \mathrm{h})$ \\
\hline 20 & 834 \\
\hline 10 & 417 (aircraft landing speed) \\
\hline 5 & 208 \\
\hline 1 & 42 \\
\hline 0.3 & 13 \\
\hline 0.1 & 4 \\
\hline
\end{tabular}




\subsubsection{Loading Mode}

As discussed in Chapter 3, many loading modes have been used (as seen in the literature) to conduct tests for measuring AC stiffness. But the cyclic tension-compression mode is gaining popularity due to its good representation of behaviour in the field. The selection of this mode of loading was supported by field test data in which a sinusoidal response has been observed as discussed earlier in Chapter 3.

The tension-compression test can be either based on a strain-or a stress-controlled loading mode. However, previous work performed under a stress-controlled mode has experienced difficulties in limiting displacements within the desired target range, (i.e., below what may cause permanent strain, particularly in high temperature conditions.) A key example was the work done at the University of Minnesota, where a strain magnitude of $1500 \mu \mathrm{m} / \mathrm{m}$ was obtained at test temperatures between 40 and $50^{\circ} \mathrm{C}$ [59]. Under similar test conditions performed in this study, such values of strain resulted in a non-linear response.

Since by definition the dynamic complex modulus should be measured within the linear viscoelastic range, the strain-controlled mode was chosen in this study for its practicality and for the fact that the applied displacements will produce a linear response.

\subsubsection{Establishment of the Appropriate Strain Magnitude}

To determine the magnitude of displacements that could be used safely in this study in performing the complex modulus test, (i.e., without inducing a non-linear viscoelastic response, a linearity investigation was performed where different displacement magnitudes were applied and the results were examined to determine the level where 
permanent strain occurred.) The objective was to select the maximum displacement that will not result in a permanent strain and to avoid extremely small displacements that could result in energy loss. With energy loss expected at the joints and connections, and because of limitations of the available sensors tracing the different responses, small strain values will make it difficult to properly and accurately measure the targeted response. Linearity checks were continued on all other mixes included in the main investigation performed using the developed test protocol.

The displacement induced by the strain-controlled loading mode was detected by the extensometer as shown in Figure 4.3. The strain was calculated using the initial gauge length (distance between the knives of the gauge) and the instantaneous displacements measured by the extensometer.

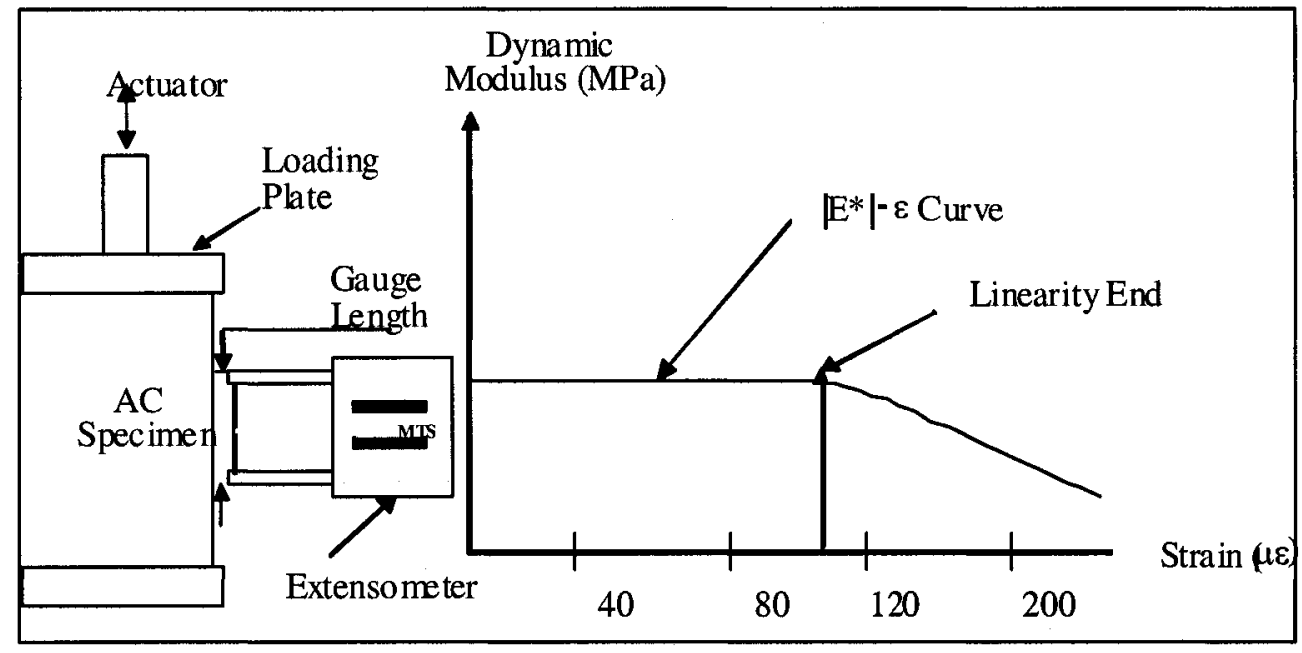

Figure 4.3. Linearity check concept

Table 4.3 shows the tests designed to facilitate the linearity check investigation using a SuperPave mix (SP 2) prepared with a PG 52-34 binder and performed at two temperatures $\left(+10^{\circ} \mathrm{C}\right.$ and $\left.+25^{\circ} \mathrm{C}\right)$. Tests were performed at different frequencies including 20, 10, 5, 1, 0.3 and $0.1 \mathrm{~Hz}$. The results are discussed in Chapter 5. 
Table 4.3. Tests designed to check the linearity of the AC response

\begin{tabular}{|c|c|}
\hline $\begin{array}{c}\text { Displacement } \\
\text { (micron) }\end{array}$ & $\begin{array}{c}\text { Corresponding Strain } \\
(\mu \varepsilon)\end{array}$ \\
\hline 1 & 40 \\
\hline 2 & 80 \\
\hline 3 & 120 \\
\hline 4 & 160 \\
\hline 5 & 200 \\
\hline 6 & 240 \\
\hline 7 & 280 \\
\hline
\end{tabular}

\subsubsection{Test Set-up and Data Acquisition System}

After defining input and output test parameters, efforts were then focused on developing the test setup for effectively conducting the complex modulus test. The design of the test set-up included identifying the components that needed to be assembled including hardware and software necessary for running the test. The following items were considered to ensure an effective test protocol capable of achieving the test objectives:

- Anticipating a maximum stress magnitude at low temperatures equivalent to $6 \mathrm{MPa}$, a hydraulic actuator capable of loading the $100-\mathrm{mm}$ diameter specimen was used. An electronic load cell with a capacity of $100 \mathrm{kN}$ was used to measure the applied load required to produce the assigned displacement magnitude. Initially, the load cell was placed in contact with the moving actuator just above the upper adaptor. However, it has been discovered that the load cell was unable to record accurately measurements of the applied force when it was moving with the hydraulic actuator. The inaccuracy of the force amplitude recorded was significantly high at a high rate of loading frequencies (10 and $20 \mathrm{~Hz}$ ). Accordingly, the load cell was positioned in contact with the lower hardened steel disc beneath the specimen. Force measurements were found to be more accurate when the load cell was not in contact with the moving actuator. 
- Since a strain-controlled mode was selected to perform the complex modulus test, a strain gauge capable of recording the targeted displacements and providing the necessary signals needed to control crosshead movements was used. Accordingly, extensometers with high accuracy (MTS product Model 632.11F-90) such as that shown in Figure 4.4 were used. The extensometers were mounted on the side of the specimen with springs provided by the manufacturer. However, slippage at contact points between the sample and the knives of the extensometer was observed during the test; this affected the accuracy of the measured strains. Moreover, several tests were interrupted owing to breakage of specimens due to unexpected movement of the actuator caused by slippage of the extensometers, particularly at high frequencies and low temperatures. To resolve this problem, a drop of five-minute epoxy was used to back up the springs in holding the extensometers in place.

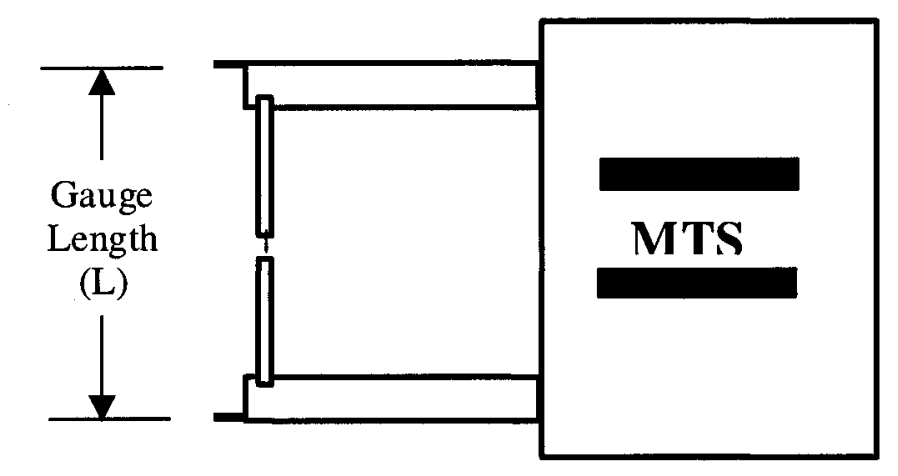

Figure 4.4. Axial extensometer Model 632.11F-90

- All sensors (the load cell and the controller strain gauge) connected to the system were calibrated to ensure that outputs accurately represent the physical condition sensed by the device (e.g., displacement or force).

- Tuning was performed on the assigned control mode to optimize the test performance by minimizing system errors and to ensure that the system is producing the exact 
value of displacements and in clear signals. The extensometer, used in controlling the applied strain, was tuned according to the manufacturer's specifications. The improper selection of tuning parameters could result in inaccurate test results, as will be explained in Chapter 5. Tuning involved changing parameters entitled "Gains." These are system control factors that compensate for changes in the material characteristics. The different Gains used in this investigation included:

Proportional Gain: Referred to as the "P Gain," which, when increased, the error in the signal decreases and the feedback signal tracks the command signal more closely.

Integral Gain: Referred to as "I Gain." It increases the system response during static or low-frequency operations and maintains the mean level during highfrequency operations.

Derivative Gain: Entitled "D Gain." It introduces a derivative of the feedback signal. It helps the system to anticipate the feedback signal's rate of change and slows the system's response at high rates of change. It is an essential tool for dynamic tests.

Feed Forward Gain: Called the "F Gain." It helps the servocontrol loop of the system to react quickly to a sudden change in the command signal and it is again useful with dynamic tests.

Different Gains values were used at different temperatures and frequencies to accommodate the sensitivity of AC materials to these two factors. Also, different Gain values were used with different mix types to capture the sensitivity of the $\mathrm{AC}$ mechanistic 
response to mix type. Higher values of tuning parameters were used with low temperatures and high frequencies to compensate for stiffer material at this testing condition.

Changing the tuning parameters was achieved by incorporating two predominant Gains, which are responsible for optimizing system performance in the complex modulus test, namely the P and I Gains. Other tuning parameters, such as the D and F Gains, do not have a noticeable effect on tension-compression tests. However, they are very useful in dynamic tests. Table 4.4 shows ranges of tuning parameter values used in this study.

Table 4.4. Typical extensometer tuning parameters for $P$ and I Gains

\begin{tabular}{|c|c|c|c|c|}
\hline Temperature $\left({ }^{\circ} \mathrm{C}\right)$ & P Gain & I Gain & D Gain & F Gain \\
\hline-10 & $1700-1450$ & $170-145$ & 0.120 & 0.0133 \\
\hline 0 & $1550-1350$ & $155-135$ & 0.120 & 0.0133 \\
\hline 20 & $650-150$ & $65-15$ & 0.120 & 0.0133 \\
\hline 30 & $380-30$ & $38-3$ & 0.120 & 0.0133 \\
\hline 40 & $200-10$ & $20-1$ & 0.120 & 0.0133 \\
\hline
\end{tabular}

\section{- Computer Controlled Software}

Because of the large number of test parameters that need to be traced at each test temperature and frequency, the test procedure was automated employing a computer program. A built-in software entitled "TestStar II," provided by the manufacturer, was used to establish a computer program "MultiPurpose Testware (MPT)" that is responsive to test requirements [MTS, Model 793.00 System Software, Version 3.1]. The MPT software defines activities and sequencing involved in running the test including the following:

- Drive the actuator and cause it to move according to specific input

- Monitor and act on real-time sensor values as the test progresses 
- Acquire test data and wire them to disk

- Receive and send information to external devices on the test station

- Play out profiles

More details of parameters composing the MPT are provided below:

- Rest Period. A rest period between any two consecutive loading frequencies was important to enable the testing machine to release the force applied during a frequency before proceeding to the next loading frequency. This is true particularly when high stresses are observed such as the case at low temperatures. Accordingly, a two-minute rest period was assigned after 20 and $10 \mathrm{~Hz}$ for testing at temperatures of -10 and $0^{\circ} \mathrm{C}$. However, a rest period of one minute was assigned after $5,1,0.3$, and $0.1 \mathrm{~Hz}$ for tests performed at -10 and $0^{\circ} \mathrm{C}$. Also, a rest period of one minute was assigned after all frequencies for testing at temperatures of 20,30 , and $40^{\circ} \mathrm{C}$.

- Sampling Rate. Tracing a clear signal of waveform cycles requires massive data. A sampling rate of 100 points per cycle was found to be enough for accurately plotting sinusoidal signals capturing effectively peak stress and strain values. Accordingly, this sampling rate was selected to collect data of inputs and outputs for the complex modulus test.

- Number of Cycles. The number of cycles was determined based on the stage in the test at which stable signals are obtained. It differs from one frequency to another and from one temperature to another. Table 4.5 shows the number of cycles used to conduct a complex modulus test at different temperatures and 
frequencies. It can be noticed that the number of cycles required to reach the stabilization state at cold temperatures was greater than the number of cycles required at warm temperatures. Moreover, the number of cycles required to reach the stabilization state at high frequencies was greater than the number of cycles required at low frequencies. This phenomenon reflects the sensitivity of $\mathrm{AC}$ materials to these two factors (temperature and frequency).

Table 4.5. Number of cycles for all temperature conditions and frequencies

\begin{tabular}{|c|c|c|}
\hline Frequency $(\mathrm{Hz})$ & \multicolumn{2}{|c|}{ Number of Cycles } \\
\cline { 2 - 3 } & \multicolumn{2}{|c|}{ Temperature $\left({ }^{\circ} \mathrm{C}\right)$} \\
\cline { 2 - 3 } & $-10,0$ & $20,30,40$ \\
\hline 20 & 1000 & 700 \\
\hline 10 & 500 & 300 \\
\hline 5 & 250 & 150 \\
\hline 1 & 150 & 60 \\
\hline 0.3 & 50 & 10 \\
\hline 0.1 & 10 & 10 \\
\hline
\end{tabular}

- Type of Data Collection. There are many methods of data collection, but two methods are appropriate for a test consisting of several frequencies, namely the linear and the circular. The circular buffer type has an advantage over the linear one in collecting the last few cycles according to a user preference. The linear buffer type records all data with no exception. However, the circular type overwrites data on the predetermined size of the buffer. Based on the selected sampling rate and number of cycles, data collected for the whole sweep of frequencies exceeded the capacity of the spreadsheet which contains 65536 lines. To provide sufficient space for collecting essential data for cycles with stabilized signals, a circular type of data collection was used with a buffer size of 5000 points. 


\section{- Data Acquisition System}

The data acquisition system was designed to enable automatic collection of data needed for characterization including real time, applied strain as measured by the two extensometers, and the resulting force detected by the load cell. Raw data were wired automatically to a folder containing a spreadsheet file.

\subsubsection{Data Reduction Procedure}

Processing manually the massive amount of data generated by the test system, associated with the combination of temperatures and frequencies, was found to be a tedious and time-consuming task. To overcome this difficulty, a data reduction system was developed consisting of three major operations as described in the following paragraphs. The flow chart in Figure 4.5 summarizes these three operations.

Excel Macro: A macro program based on Microsoft Excel was developed and is being used at NRC Canada for preliminarily data processing. The massive amount of raw data recorded during the test is reduced by the macro to calculate preliminarily stress and strain amplitudes. The macro picks up data from the last three cycles of each frequency to run the calculations. It was intended to consider only data from these cycles among those reached after the stabilization stage. However, this macro was less successful in calculating accurately the phase angles; hence, a second data reduction process was implemented in further processing using the commercial statistical package entitled "TableCurve."

TableCurve. This is a statistical package, which was used to assist in fitting data to curves of the user selection and calculates the coefficients composing the selected curve. The 
TableCurve was used to process data, which was preliminarily processed in the previous stage using the macro, to calculate amplitudes and phase angles of stresses and strains for the whole sweep of temperatures and frequencies. The TableCurve fits the data in a waveform equation (sinusoidal) to calculate coefficients of Equations 4.5 and 4.6:

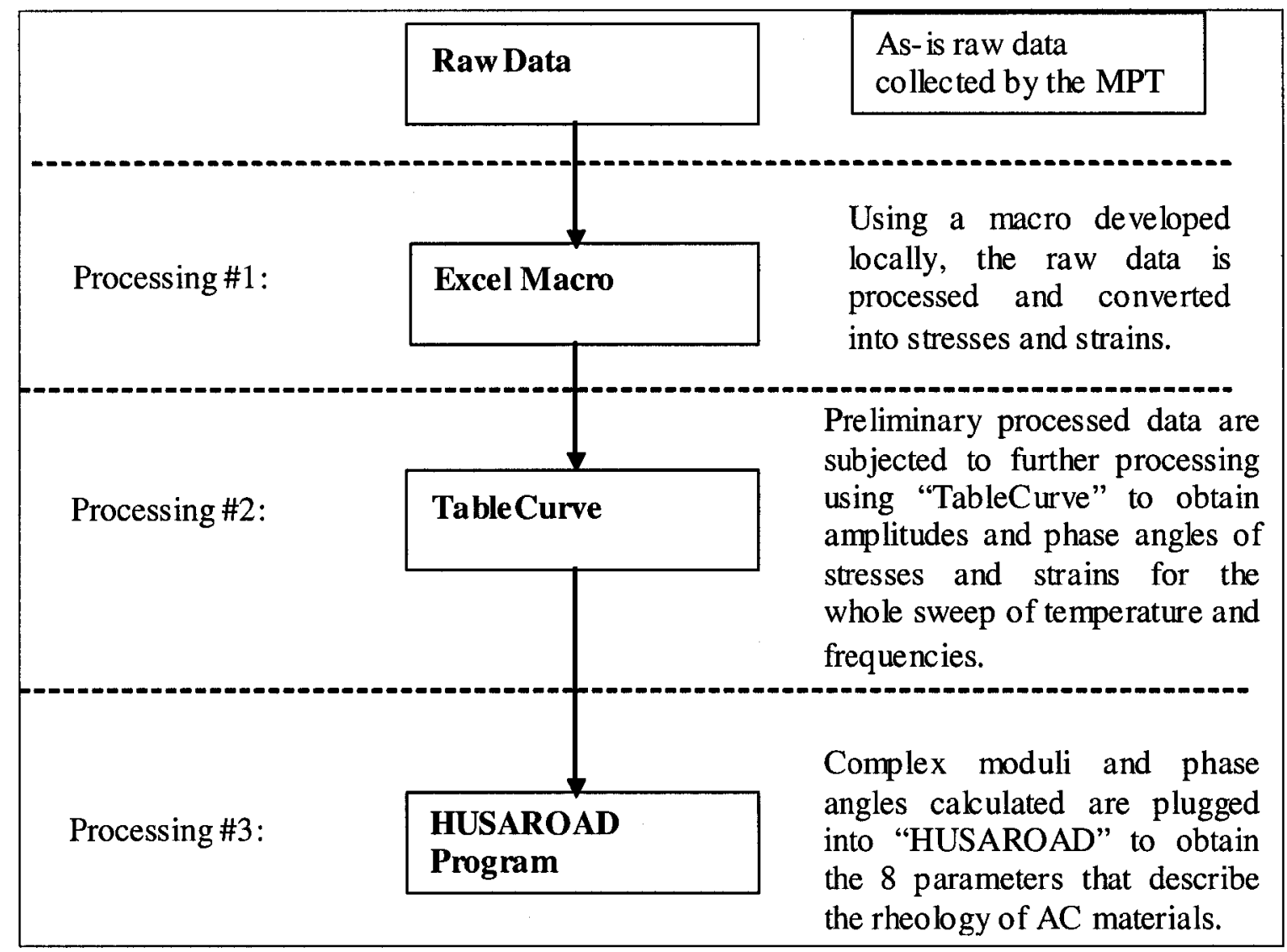

Figure 4.5. Data reduction procedure

$$
\begin{aligned}
& \sigma=a_{1}+b_{1} \sin \left(2 \pi t / d_{1}+c_{1}\right)- \\
& \varepsilon=a_{2}+b_{2} \sin \left(2 \pi t / d_{2}+c_{2}\right)
\end{aligned}
$$

where: $\sigma$ and $\varepsilon$ are the stress and strain respectively at time $\mathrm{t}$,

$b_{I}$ and $b_{2}$ are the amplitude of stress and strain respectively, 
$a_{1}$ and $a_{2}$ are regression constants,

$c_{1}$ and $c_{2}$ are individual phase angles of stress and strain wave functions respectively.

The phase lag $\phi$ between stress and strain cycles was calculated as the difference of $c_{l}$ and $c_{2}$ in radian.

Only coefficients that were used to calculate the complex modulus and the lag phase were considered. Figure 4.6 is a typical curve-fitting process applied on strain data collected from a test performed using a HMA 2 at a frequency of $1 \mathrm{~Hz}$.

HUSAROAD Program. The HUSAROAD is a module of the VEROAD Program [43]. HUSAROAD software was used to yield the Huet-Sayegh model parameters from the complex moduli and phase angles obtained experimentally. The master curve for the complex moduli and phase angles could also be obtained using HUSAROAD.

The determination of Huet-Sayegh model parameters was based on non-linear least square methods. The best fit between the measured data at one hand and the values calculated by the model on the other was found when the sum of squares was minimal. The program provides both a visual drawing and numerical results of the two diagrams mentioned in Chapter 3 (Cole-Cole plot and Black space) to assist in obtaining the sum of least squares.

\subsection{Design of AC Characterization Technique}

The characterization of asphalt concrete was performed under two main categories, namely the conventional physical characterization approach used currently by the industry and the mechanistic characterization technique proposed for improving analysis and design of roadway pavement. The new characterization technique was not intended 
to completely replace physical characterization, but is intended to support elaborate design requirements especially in critical designs (heavily trafficked roads). There are trends in the industry to link physical characterization to the mechanistic response to facilitate the continuation of the current practice when facilities for the mechanistic tests are not available. These two techniques are discussed in the following subsections.

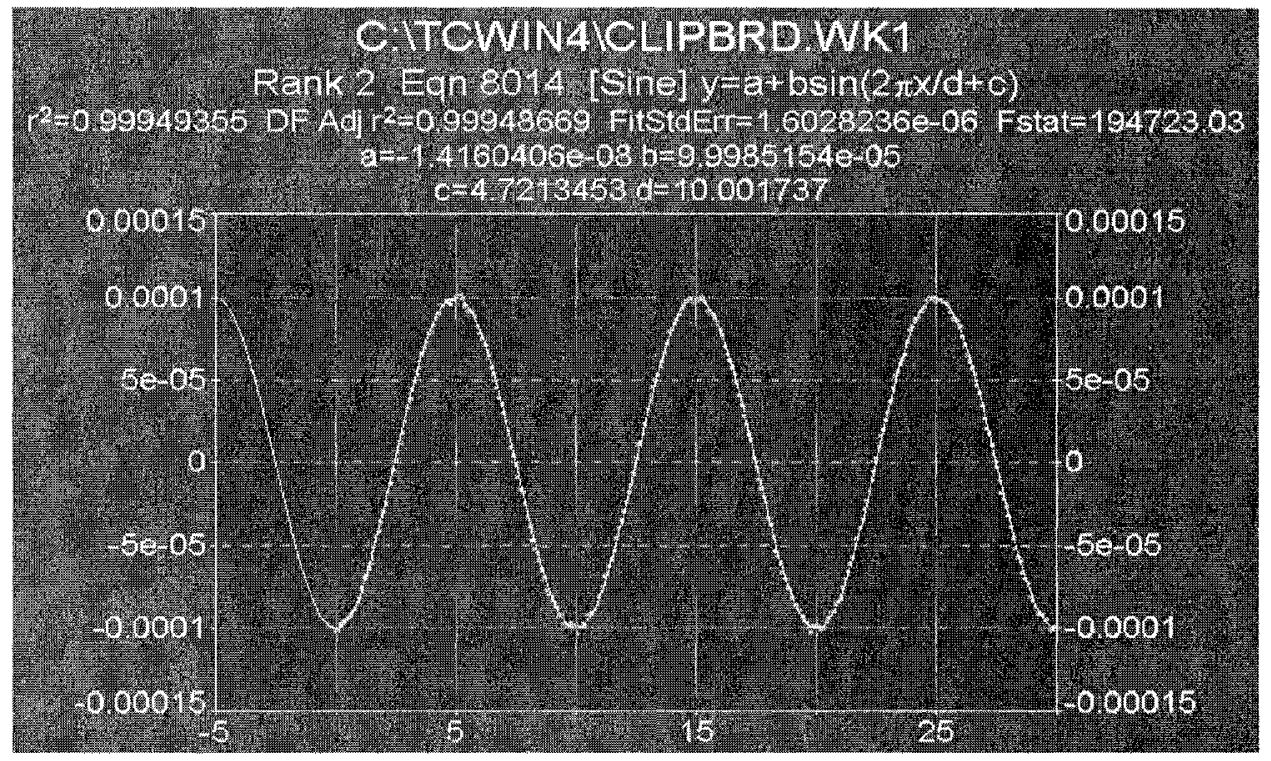

Figure 4.6. Data reduction procedure using TableCurve

\subsubsection{Conventional Physical Characterization of AC Materials}

Physical characterization of $\mathrm{AC}$ materials involves proportioning of mineral aggregates, determining binder characteristics, performing mix designs, and conducting some standard physical tests to evaluate the quality of the mix produced. Components of the physical characterization performed in this study are discussed in the following paragraphs: 


\section{- Aggregates Proportioning}

Sieve analysis was performed in the National Research Council Canada (NRC) laboratories on aggregates provided by a local supplier using the Rideau Quarry, located in Ottawa, Ontario. Ontario Ministry of Transportation (MTO) specifications [71] were followed, which are originally based on AASHTO standards.

Two categories were used to identify the aggregate size:

Nominal maximum size defined as the size of one sieve larger than the first sieve to retain more than $10 \%$

Maximum size defined as the size of one sieve larger than the nominal maximum size Six aggregate stockpiles, of the properties shown in Table 4.6, were used according to the nominal maximum aggregate size. Results of sieve analysis performed on all aggregates used in this study are shown in Table 4.7.

The adopted local names are based on the type of mix where these aggregates are commonly used. The F3 fraction contained only fine dust passing sieve \# $200(0.075$ $\mathrm{mm})$. This fraction was useful in achieving gradation requirements and particularly in designing the job formula for SuperPave mixes, which involve an open-graded mix design with a high fine material content.

Table 4.6. Aggregate fraction used in this study

\begin{tabular}{|c|c|c|}
\hline $\begin{array}{c}\text { Aggregate } \\
\text { Designation }\end{array}$ & Ontario Local Name & $\begin{array}{c}\text { Nominal Maximum } \\
\text { Aggregate Size (mm) }\end{array}$ \\
\hline CA 1 & HL8 Stone & 19 \\
\hline CA 2 & HLA Stone & 16 \\
\hline CA 3 & HL3 Stone & 13.2 \\
\hline F1 & Regular Dust & 9.5 \\
\hline F 2 & Natural sand & 1.18 \\
\hline F 3 & Mineral Filler & - \\
\hline
\end{tabular}


Table 4.7. Results of sieve analysis conducted on six aggregate fractions

\begin{tabular}{|c|c|c|c|c|c|c|}
\hline \multirow{3}{*}{ Sieve Size (mm) } & \multicolumn{7}{|c|}{ \% Passing } \\
\cline { 2 - 7 } & CA1 & CA2 & CA3 & F1 & F2 & F3 \\
\hline 26.5 & - & - & - & - & - & - \\
\hline 19 & 86.7 & - & - & - & - & - \\
\hline 16 & 59.7 & 95.7 & - & - & - & - \\
\hline 13.2 & 30.4 & 59.5 & 93.7 & - & - & - \\
\hline 9.5 & 7.3 & 7.9 & 48.3 & - & - & - \\
\hline 4.75 & 2.2 & 3.4 & 2.3 & 90.8 & 99.9 & - \\
\hline 2.36 & 1.1 & 2.4 & 0.6 & 61.6 & 98.9 & - \\
\hline 1.18 & 0.7 & 2 & 0.6 & 41.3 & 94.4 & - \\
\hline 0.6 & 0.5 & 1.7 & 0.5 & 28.3 & 41.5 & - \\
\hline 0.3 & 0.4 & 1.3 & 0.5 & 17.6 & 10.5 & - \\
\hline 0.15 & 0.3 & 1 & 0.4 & 9.4 & 2.4 & - \\
\hline 0.075 & 0.1 & 0.2 & 0.1 & 1.2 & 0.5 & - \\
\hline
\end{tabular}

\section{- Binder Characteristics}

Asphalt cements used as binders in the designed mixes were classified by suppliers according to the Performance Grade (PG) specifications, now widely used by the road industry in North America. Moreover, engineered binders are commonly used to enhance the performance of asphalt concrete by reducing the potential for rutting and cracking. PG grades with a relatively high initial number are needed for increasing the resistance to deformations at relatively high temperatures. The second number in the PG grade indicates resistance to cracking at relatively low temperatures [72].

Three binder types - two neat binders and an engineered binder - were used to prepare all AC specimens for the complex modulus test. These were PG 58-22, PG 52-34, and PG 64-34 respectively. However, the engineered binder PG 64-34 was not used to prepare samples of HMA1 and SP1 since these two mixes are commonly used as binder courses. It is not economically justifiable to use such a relatively expensive binder in these layers 
since exposure to the environment and traffic loading is less severe compared with surface layers.

The binder types mentioned above were provided by McAsphalt Industries Ltd (Ontario, Canada). Table 4.8 shows properties of the binders as provided by the manufacturer. These properties were determined based on the tests mentioned in Chapter 2, Section 2.4.2 of this thesis.

Table 4.8.Binder properties as provided by the manufacturer

\begin{tabular}{|c|c|c|c|c|}
\hline Tests & \multicolumn{3}{|c|}{ Test Results } & \multirow{2}{*}{$\begin{array}{c}\text { AASTHO } \\
\text { Specifications }\end{array}$} \\
\hline Tests on unaged material & PG 58-22 & PG 52-34 & PG 64-34 & \\
\hline Brookfield, $135^{\circ} \mathrm{C}, \mathrm{mPa} . \mathrm{s}$ & 0.323 & 0.266 & 1.053 & $3.0 \max$ \\
\hline Flash Point $\mathrm{COC},{ }^{\circ} \mathrm{C}$ & $230+$ & 230 & 230 & $230 \mathrm{~min}$ \\
\hline $\mathrm{G}^{*} / \mathrm{Sin} \delta @ 58^{\circ} \mathrm{C}, 10 \mathrm{rad} / \mathrm{sec}, \mathrm{kPa}$ & 1.518 & 1.233 & 1.329 & $1.0 \mathrm{~min}$ \\
\hline Tests on RTFO Residue & PG 58-22 & PG 52-34 & PG 64-34 & \\
\hline \% Loss Weight & 0.212 & 0.329 & -0.364 & $1.0 \max$ \\
\hline $\mathrm{G}^{*} / \mathrm{Sin} \delta @ 58^{\circ} \mathrm{C}, 10 \mathrm{rad} / \mathrm{sec}, \mathrm{kPa}$ & 4.123 & 2.912 & 2.446 & $2.2 \mathrm{~min}$ \\
\hline $\begin{array}{c}\text { Tests on PAV (after RTFO) }\left(\text { Run @ } 100^{\circ} \mathrm{C}\right) \\
\text { Residue }\end{array}$ & PG 58-22 & PG 52-34 & PG 64-34 & \\
\hline $\mathrm{G}^{*} \mathrm{x} \operatorname{Sin} \delta @ 22^{\circ} \mathrm{C}, 10 \mathrm{rad} / \mathrm{sec}, \mathrm{kPa}$ & 2837 & 2083 & 1822 & $5000 \max$ \\
\hline Creep Stiffness@ $-12^{\circ} \mathrm{C}, \mathrm{S}, 60 \mathrm{sec}, \mathrm{MPa}$ & 125.0 & 222 & 125 & $300 \max$ \\
\hline Slope @ $-12^{\circ} \mathrm{C}, \mathrm{m}, 60 \mathrm{sec}$ & 0.361 & 0.312 & 0.325 & $0.300 \mathrm{~min}$ \\
\hline Other Properties & PG 58-22 & PG 52-34 & PG 64-34 & \\
\hline Bulk Specific Gravity & 1.025 & 1.020 & 1.028 & \\
\hline Mixing Temperature, ${ }^{\circ} \mathrm{C}$ & 155 & 148 & 160 & \\
\hline Compaction Temperature, ${ }^{\circ} \mathrm{C}$ & 148 & 133 & 155 & \\
\hline
\end{tabular}

\section{- Mix Designs}

The aggregate fractions were proportioned to achieve design requirements of three types of conventional hot mix asphalt (HMA) based on the Marshall approach and two SuperPave mixes according to ASSHTO specification generated based on SHRP technology. The function of these five mixes conforms to the common applications indicated in Table 4.9. The aggregate fractions and the selected combinations used in this study cover a wide range of mix types that are commonly used by the industry. The 
mechanical tests performed on the $\mathrm{AC}$ mixes reflected well the impact of aggregate components of the tested mixes as will be discussed in Chapter 6 .

The intended function of the layer determines the shape of the curve. For example, the SP 2 curve was located entirely above the maximum density line. This meant that the designed SP 2 mix was a fine open-graded mix, which reflects its function as the driving layer with a smooth surface for the driver's convenience and an ability to sustain snow removal operations without the potential for damage but with adequate skid resistance [73]. Table 4.10 shows aggregates and combination ratios used to satisfy aggregate specification for the different mixes used in this investigation.

Table 4.9. Designations of mixes used in this study

\begin{tabular}{|c|c|c|c|}
\hline Mix Designation & MTO Designation & Function in Road Structure & $\begin{array}{c}\text { Nominal Maximum } \\
\text { Aggregate Size(mm) }\end{array}$ \\
\hline HMA 1 & HL8 & Stabilized base course & 19 \\
\hline HMA 2 & HL4 & Binder course layer & 16 \\
\hline HMA 3 & HL3 & Surface layer & 13.2 \\
\hline SP 1 & - & Binder course layer & 19 \\
\hline SP 2 & - & Surface layer & 12.5 \\
\hline
\end{tabular}

The MTO specifications [71] were followed to determine the appropriate combinations of different aggregate fractions to achieve the job mix formulae that satisfy gradation requirements outlined for each specific HMA mix type. The AASHTO specifications were followed to create job mix formulae that satisfy the gradation curves of the two SuperPave mix designs [21]. The gradation curves are illustrated in Figures 4.7 to 4.11 . The gradation curves of all mixes fit well within the limits set for these mix types. 
Table 4.10. Selected aggregate fractions

\begin{tabular}{|l|l|l|l|l|l|l|}
\hline \multirow{2}{*}{ Mix Type } & \multicolumn{7}{|c|}{ Aggregates Fractions (\%) } \\
\cline { 2 - 7 } & CA 1 & CA 2 & CA 3 & F 1 & F 2 & F 3 \\
\hline HMA 1 & 26 & 24 & 0 & 30 & 20 & 0 \\
\hline HMA 2 & 0 & 38 & 0 & 45 & 17 & 0 \\
\hline HMA 3 & 0 & 0 & 40 & 20 & 40 & 0 \\
\hline SP 1 & 40 & 13 & 0 & 0 & 39.5 & 7.5 \\
\hline SP 2 & 0 & 21 & 0 & 74 & 0 & 5 \\
\hline
\end{tabular}

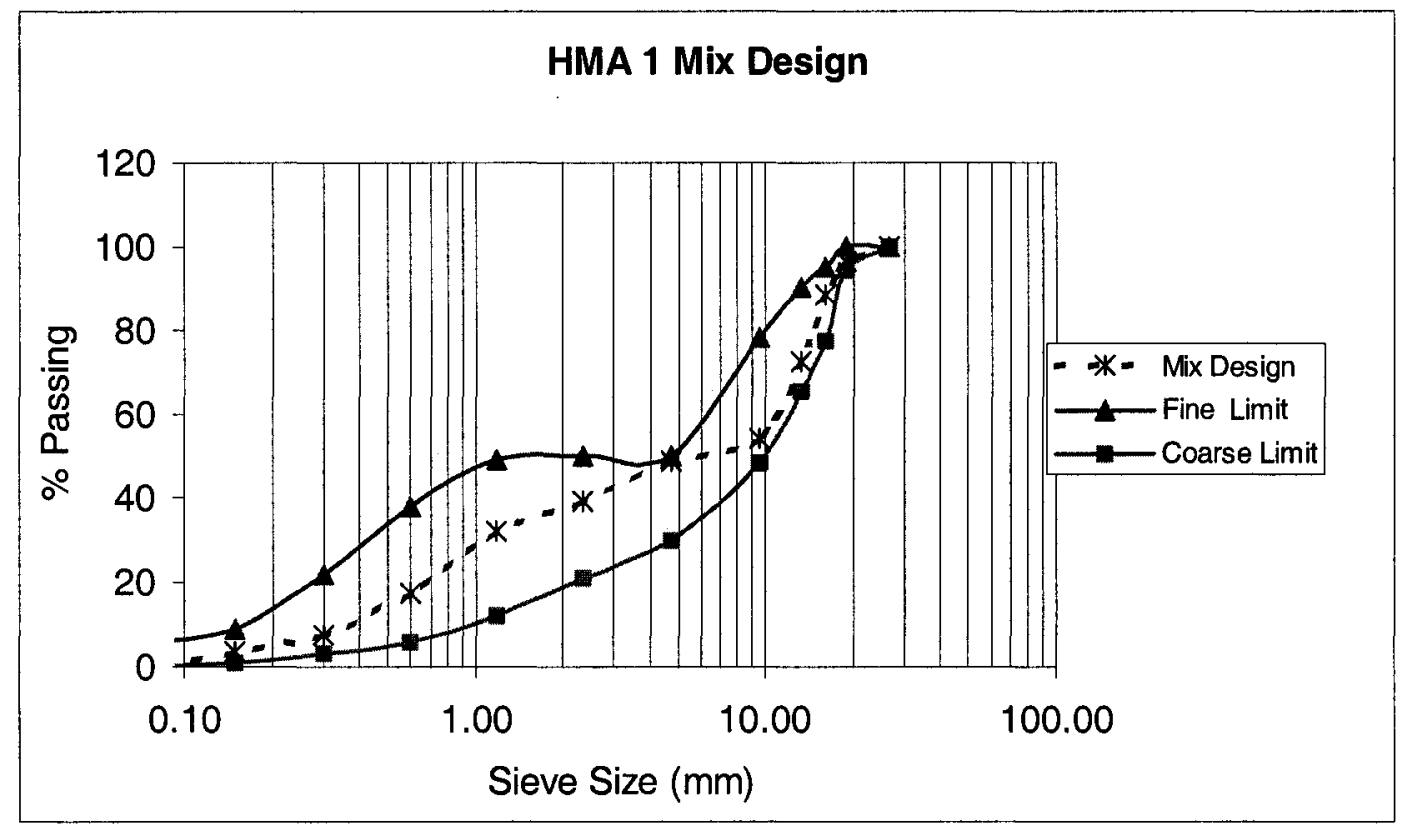

Figure 4.7.Gradation curve of aggregates used to prepare MHA 1 mix 


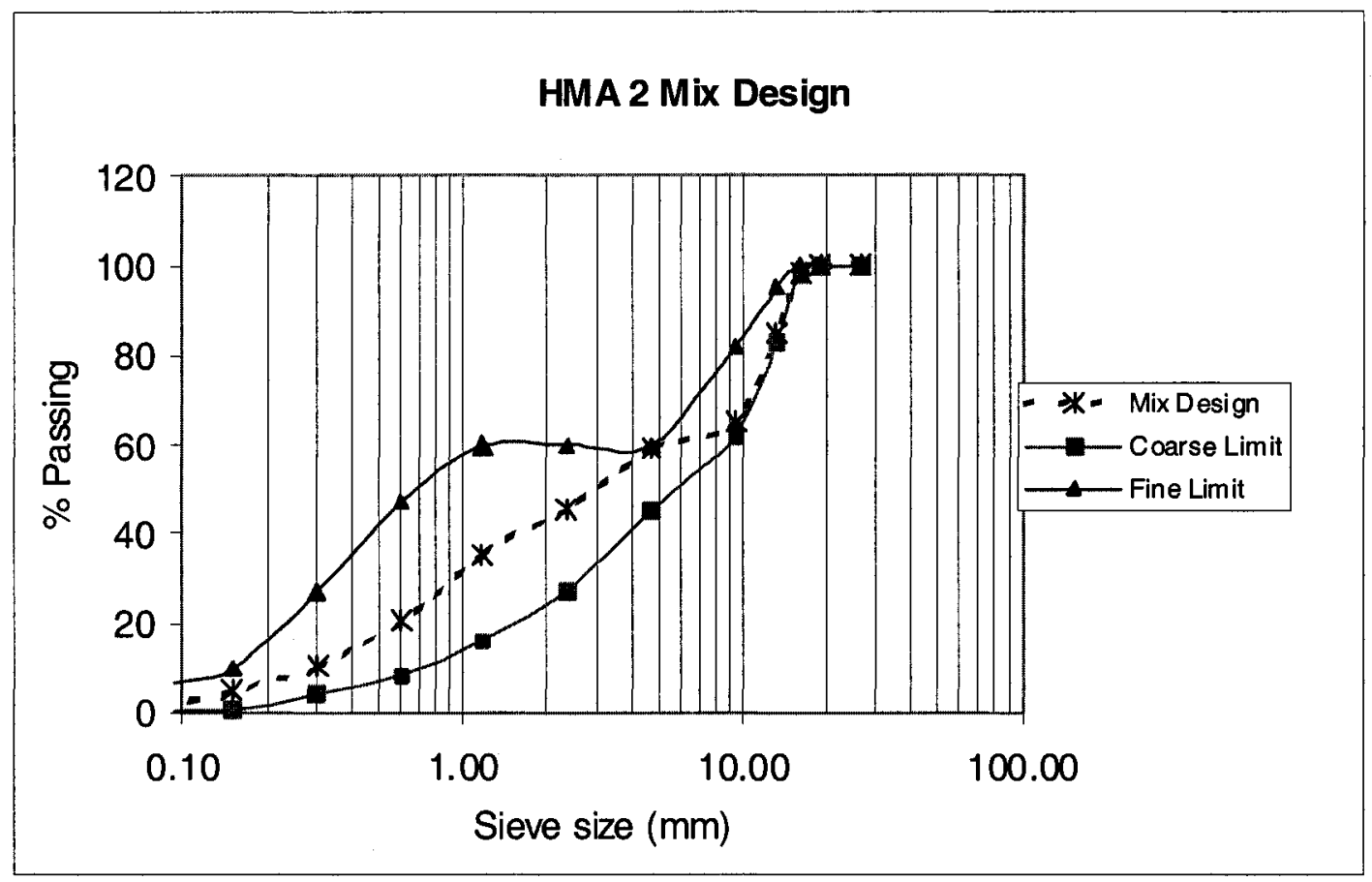

Figure 4.8.Gradation curve of aggregates used to prepare MHA 2 mix

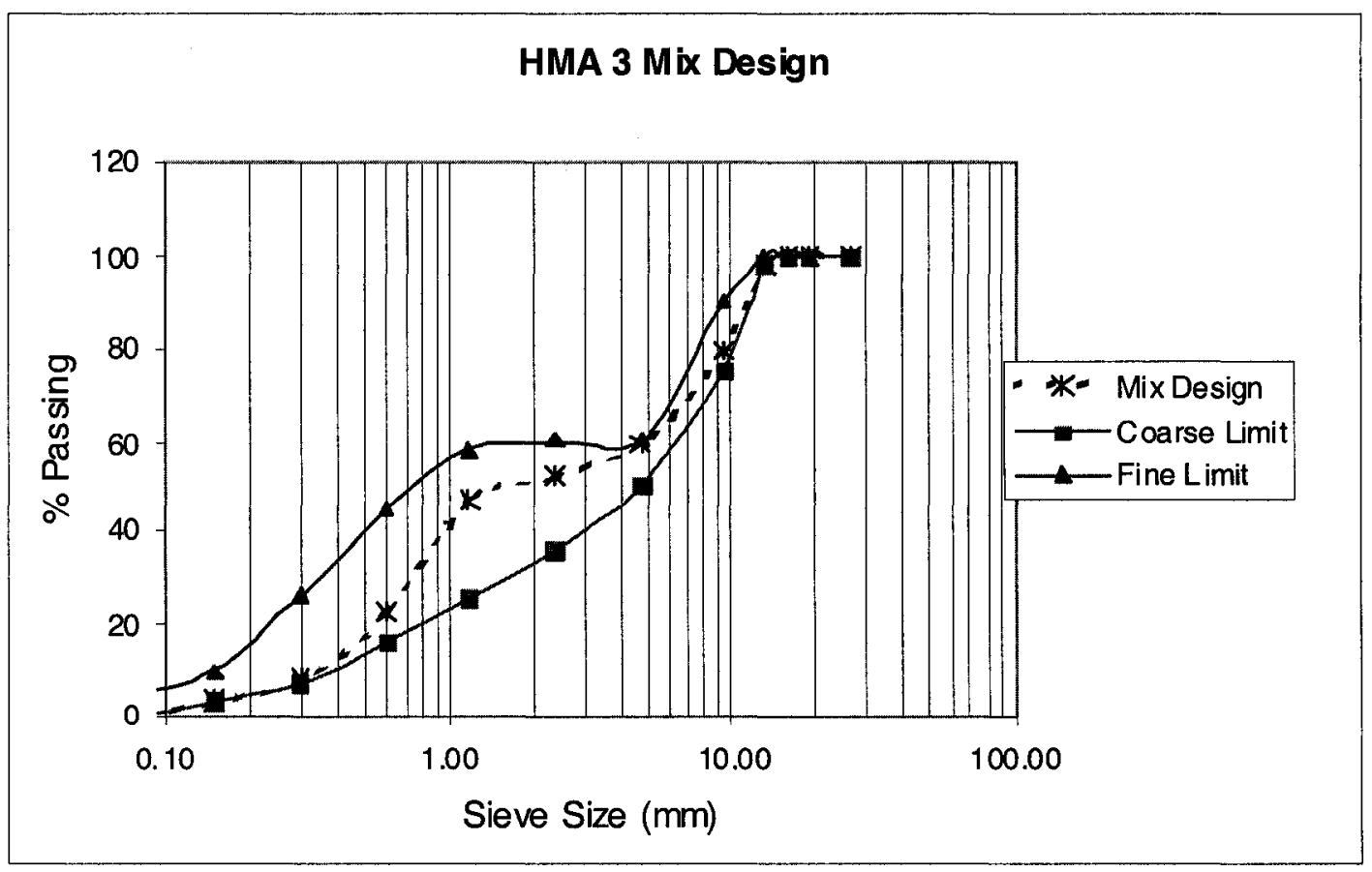

Figure 4.9. Gradation curve of aggregates used to prepare MHA 3 mix 


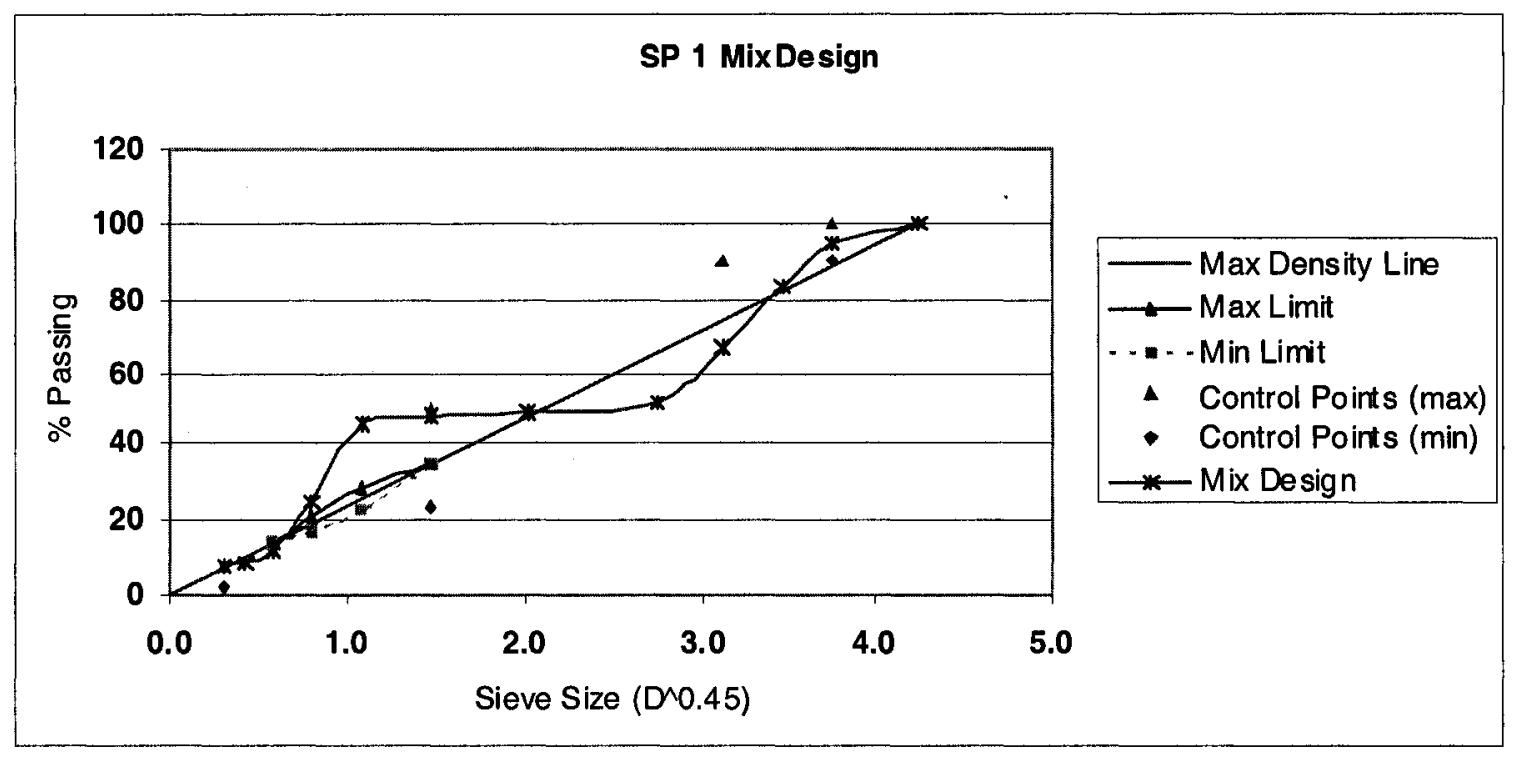

Figure 4.10.Gradation curve of aggregates used to prepare SP 1 mix

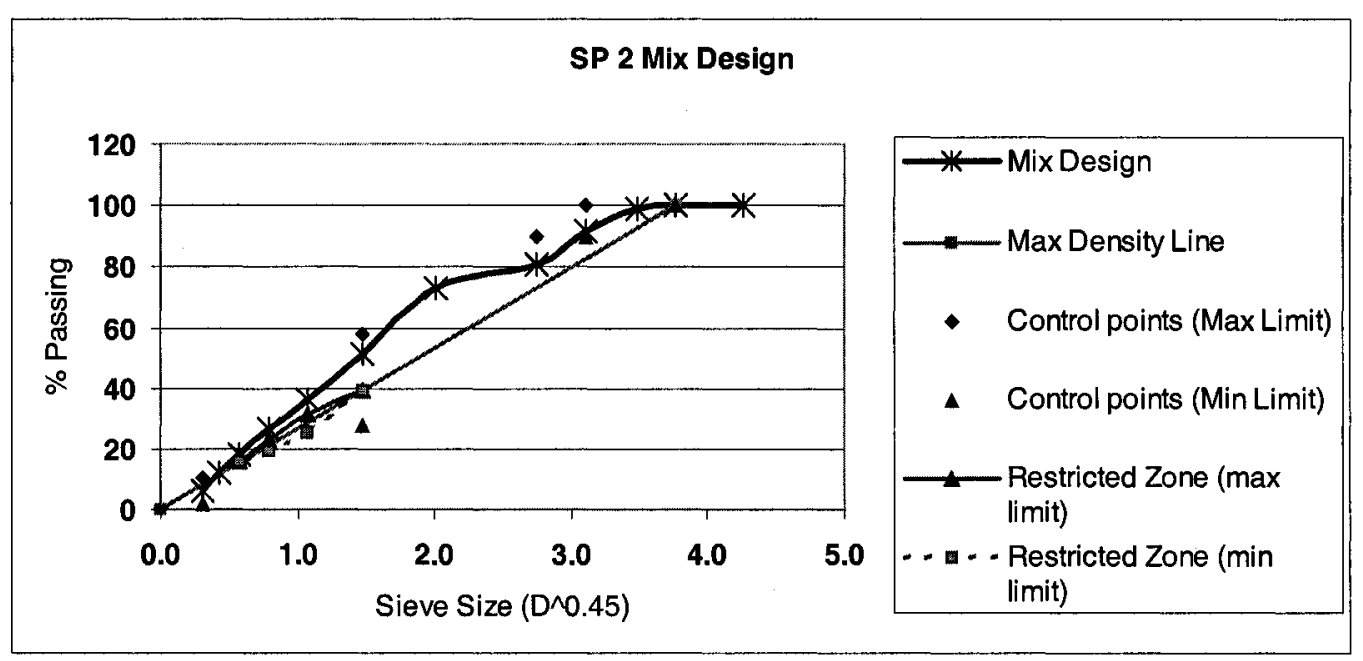

Figure 4.11.Gradation curve of aggregates used to prepare SP 2 mix

\section{- Standard Physical Tests}

Physical properties of the compacted specimens were determined in order to examine mix design conformance with MTO requirements. Tests were performed according to AASHTO specifications T 166 [68], T 209 [69] and T 269 [70] to calculate bulk specific gravity, maximum specific gravity, and air voids, respectively, for samples prepared 
using Marshall and SuperPave mix design procedures. However, a distinct plastic flow test was performed on samples prepared following the Marshall mix design procedure only to determine the Marshall Stability $(\mathrm{kN})$ and Flow $(0.25 \mathrm{~mm} @ 3.5 \%$ Air Voids) using AASHTO test Designated T 245 [25].

The AASHTO Designation P2 [25] describing a standard practice for volumetric mix design was used to evaluate physical properties of samples prepared using the SuperPave mix design procedure. The standard procedure was used to determine air voids, voids in the mineral aggregates (VMA), voids filled with asphalt (VFA), maximum relative density at the initial number of gyrations $\left(\% \mathrm{G}_{\mathrm{mm}} @ \mathrm{~N}_{\text {ini }}\right)$, the maximum relative density at the design number of gyrations $\left(\% \mathrm{G}_{\mathrm{mm}} @ \mathrm{~N}_{\mathrm{des}}\right)$ and dust to binder ratio $\left(\mathrm{P}_{0.075} / \mathrm{P}_{\mathrm{be}}\right)$. Properties of the selected mix designs are summarized in Table 4.11. Summaries of the mix design and physical properties that satisfy the Marshall and SuperPave specifications are shown in Tables 4.11 and 4.12 respectively. Typical mix design results for SP 1 and HMA 3 related to the required physical properties are plotted in Figures 4.12 and 4.13 respectively. 
Table 4.11. Marshall mix design results

\begin{tabular}{|c|c|c|c|c|c|}
\hline \multirow{2}{*}{$\begin{array}{l}\text { Mix } \\
\text { Type }\end{array}$} & \multirow[t]{2}{*}{ Mix Design Parameter } & \multirow{2}{*}{$\begin{array}{c}\text { MTO } \\
\text { Specifications }\end{array}$} & \multicolumn{3}{|c|}{ Test Results } \\
\hline & & & PG 58-22 & PG 52-34 & PG 64-34 \\
\hline \multirow[t]{5}{*}{ HMA 1} & Asphalt Content \% & $4.5-7.0$ & 4.5 & 4.5 & \\
\hline & Air Voids $\%$ & $3-5$ & 4.2 & 4.0 & \\
\hline & Marshall Stability $(\mathrm{N})$ & $8000(\min )$ & 11550 & 9220 & \\
\hline & $\begin{array}{l}\text { Marshall Flow }(0.25 \mathrm{~mm}) \\
\text { @ 3.5\% Air Voids }\end{array}$ & $8.0(\mathrm{~min})$ & 10.2 & 8.4 & \\
\hline & VMA \% & $12.5(\mathrm{~min})$ & 13.85 & 13.6 & \\
\hline \multirow[t]{5}{*}{ HMA 2} & Asphalt Content \% & $5.0-7.0$ & 5.0 & 5.0 & 5.1 \\
\hline & $\%$ Air Voids & $3-5$ & 4.6 & 4.4 & 4.5 \\
\hline & Marshall Stability (N) & 8900 (min) & 19200 & 11800 & 19000 \\
\hline & $\begin{array}{l}\text { Marshall Flow }(0.25 \mathrm{~mm}) \\
@ 3.5 \% \text { Air Voids }\end{array}$ & $8.0(\min )$ & 11.5 & 11 & 11.75 \\
\hline & VMA \% & $13.0(\mathrm{~min})$ & 13.38 & 13.04 & 13.2 \\
\hline \multirow[t]{5}{*}{ HMA 3} & Asphalt Content \% & $5.0-7.0$ & 5.0 & 5.0 & 5.0 \\
\hline & $\%$ Air Voids & $3-5$ & 4.5 & 4.5 & 4.4 \\
\hline & Marshall Stability $(\mathrm{N})$ & $8900(\min )$ & 12800 & 11450 & 11600 \\
\hline & $\begin{array}{l}\text { Marshall Flow }(0.25 \mathrm{~mm}) \\
\text { @ 3.5\% Air Voids }\end{array}$ & $8.0(\mathrm{~min})$ & 8.3 & 8.9 & 9.6 \\
\hline & VMA \% & 13.5 & 12.8 & 13.57 & 13.3 \\
\hline
\end{tabular}

Table 4.12. SuperPave mix design results

\begin{tabular}{|c|c|c|c|c|c|}
\hline \multirow{2}{*}{$\begin{array}{l}\text { Mix } \\
\text { Type }\end{array}$} & \multirow[t]{2}{*}{ Mix Design Parameter } & \multirow{2}{*}{$\begin{array}{c}\text { SuperPave } \\
\text { Specifications }\end{array}$} & \multicolumn{3}{|c|}{ Test Results } \\
\hline & & & PG 58-22 & PG 52-34 & PG 64-34 \\
\hline \multirow[t]{6}{*}{ SP 1} & $\begin{array}{l}\% \text { of binder content @ } 4.0 \% \\
\text { air voids }\end{array}$ & $3-5$ & 4.4 & 4.4 & \\
\hline & VMA \% & $14(\mathrm{~min})$ & 15.7 & 15.5 & \\
\hline & VFA \% & $65-75$ & 74.6 & 74.5 & \\
\hline & $\% \mathrm{G}_{\mathrm{mm}} @ \mathrm{~N}_{\mathrm{ini}}$ & $<90.0$ & 89.5 & 89.5 & \\
\hline & $\% \mathrm{G}_{\mathrm{mm}} @ \mathrm{~N}_{\mathrm{des}}$ & $<98.0$ & 95.5 & 95.0 & \\
\hline & $\mathrm{P}_{0.075} / \mathrm{P}_{\mathrm{be}}$ Ratio & $0.6-1.4$ & 1.11 & 1.11 & \\
\hline \multirow[t]{6}{*}{ SP 2} & $\begin{array}{l}\% \text { of binder content @ } 4.0 \% \\
\text { air voids }\end{array}$ & $3-5$ & 4.4 & 4.5 & 4.5 \\
\hline & VMA \% & $14(\mathrm{~min})$ & 14.7 & 12.8 & 14.5 \\
\hline & VFA \% & $65-75$ & 73.8 & 70.0 & 73.7 \\
\hline & $\% \mathrm{G}_{\mathrm{mm}} @ \mathrm{~N}_{\mathrm{ini}}$ & $<90.0$ & 89.0 & 89.0 & 90.5 \\
\hline & $\% \mathrm{G}_{\mathrm{mm}} @ \mathrm{~N}_{\mathrm{des}}$ & $<98.0$ & 96.7 & 96.5 & 97.0 \\
\hline & $\mathrm{P}_{0.075} / \mathrm{P}_{\mathrm{be}}$ Ratio & $0.6-1.4$ & 1.11 & 1.11 & 1.11 \\
\hline
\end{tabular}

where VMA = Voids in Mineral Aggregates 


$$
\begin{aligned}
& \text { VFA }=\text { Voids Filled with Asphalt } \\
& \left(P_{0.075} / P_{b e}\right)=\text { Dust to Binder Ratio } \\
& P_{b e}=\text { Effective Binder Content } \\
& P_{0.075}=\text { Percent of Aggregates Passing Sieve \# } 200 \\
& G_{m m}=\text { Maximum Theoretical Density (\%) } \\
& N_{\text {des }}=\text { Design Gyration Number } \\
& N_{\text {ini }}=\text { Initial Gyration Number }
\end{aligned}
$$
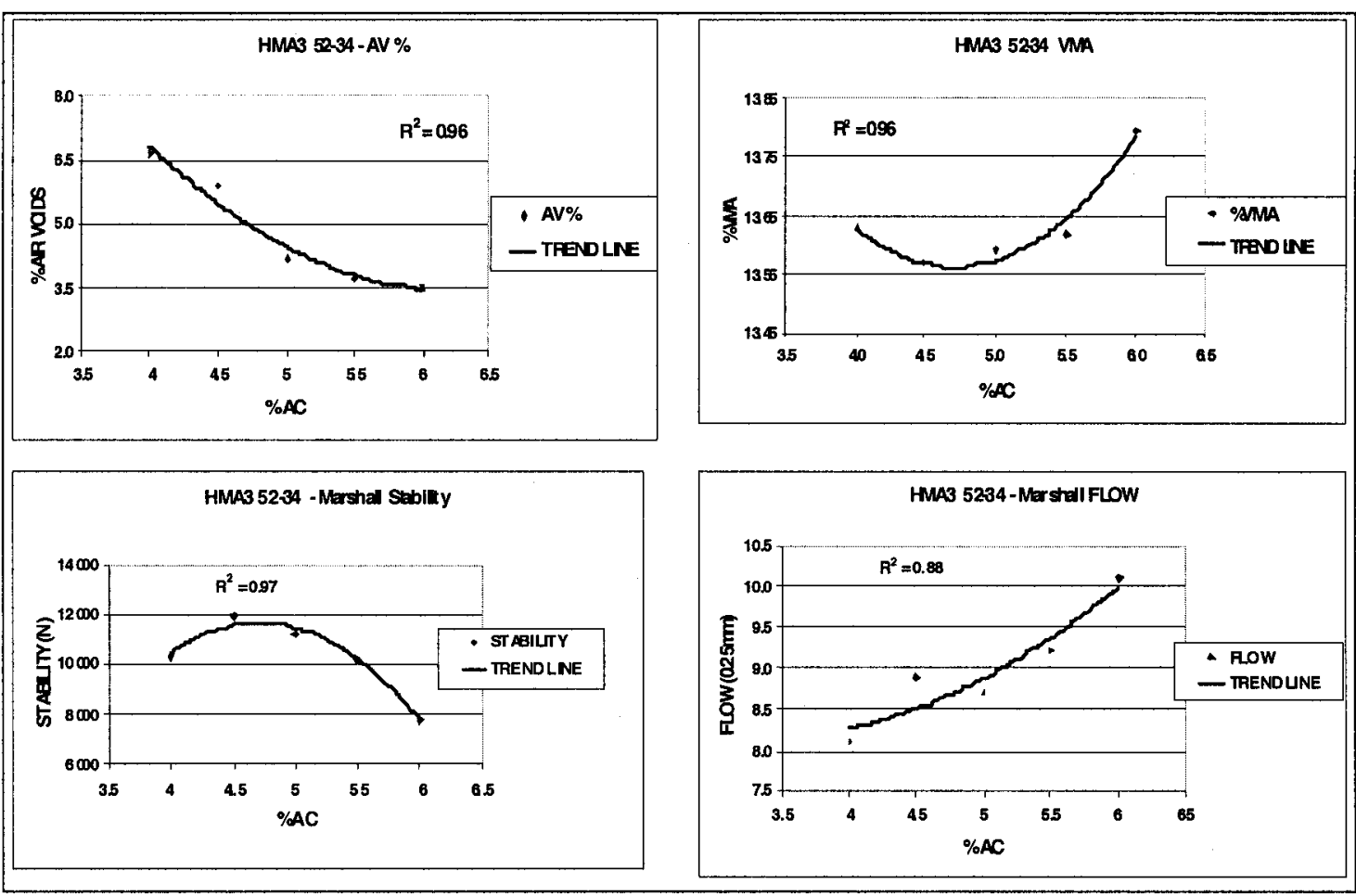

Figure 4.12.Typical results of HMA3 physical properties

Physical properties of the compacted specimens prepared for the complex modulus investigation are shown in Tables 4.13 to 4.15 . The samples are listed according to the binder type used in the mix. 

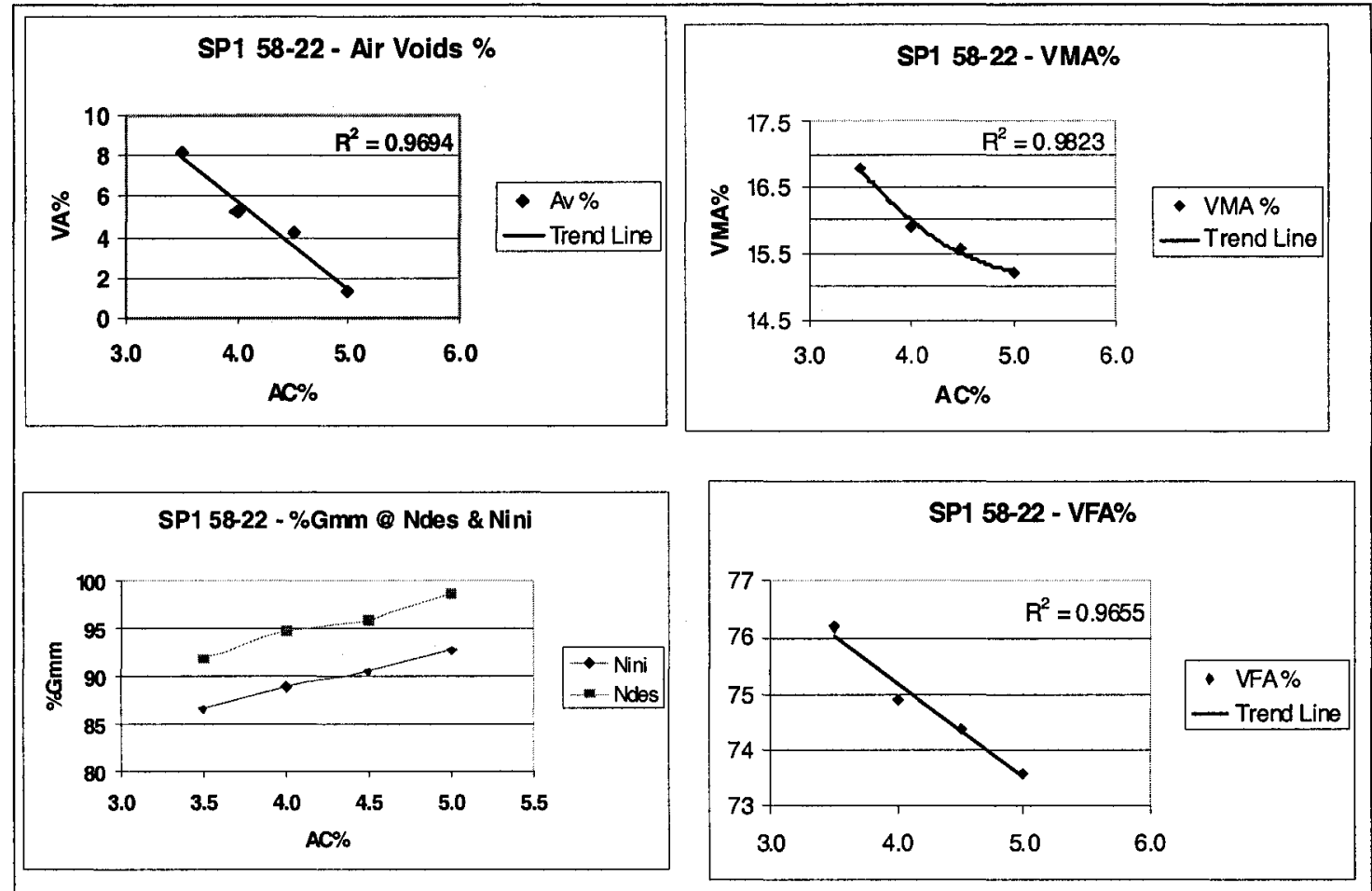

Figure 4.13. Typical results of SP 2 physical properties

Table 4.13. List of complex modulus test specimens prepared with binder PG 64-34

\begin{tabular}{|c|c|c|c|c|c|}
\hline Mix Type & $\begin{array}{c}\text { Ontario } \\
\text { Designation }\end{array}$ & $\begin{array}{c}\text { Replicate } \\
\#\end{array}$ & $\begin{array}{c}\text { Binder } \\
\text { Content }\end{array}$ & $\begin{array}{c}\text { \# Of Gyrations } \\
\text { Used }\end{array}$ & $\begin{array}{c}\text { Air Voids } \\
(\%)\end{array}$ \\
\hline HMA 2 & HL4 & 1 & \multirow{2}{*}{$5.1 \%$} & 10 & 4.7 \\
\cline { 6 - 6 } & & 2 & & 10 & 5.6 \\
\hline \multirow{2}{*}{ HMA 3 } & \multirow{2}{*}{ HL3 } & 1 & \multirow{2}{*}{$4.9 \%$} & 45 & 5.63 \\
\cline { 5 - 6 } & & 2 & & 45 & 5.37 \\
\hline \multirow{2}{*}{ SP2 } & - & 1 & \multirow{2}{*}{$4.5 \%$} & 21 & 6.0 \\
\cline { 5 - 6 } & & 2 & & 21 & 5.7 \\
\hline
\end{tabular}

Table 4.14. List of complex modulus test specimens prepared with binder PG 58-22

\begin{tabular}{|c|c|c|c|c|c|}
\hline Mix Type & $\begin{array}{c}\text { Ontario } \\
\text { Designation }\end{array}$ & $\begin{array}{c}\text { Replicate } \\
\#\end{array}$ & $\begin{array}{l}\text { Binder } \\
\text { Content }\end{array}$ & $\begin{array}{l}\text { \# Of Gyrations } \\
\text { Used }\end{array}$ & $\begin{array}{l}\text { Air Voids } \\
(\%)\end{array}$ \\
\hline \multirow[t]{2}{*}{ HMA 1} & \multirow[t]{2}{*}{ HL8 } & 1 & \multirow[t]{2}{*}{$4.5 \%$} & 17 & 5.1 \\
\hline & & 2 & & 17 & 4.5 \\
\hline \multirow[t]{2}{*}{ HMA 2} & \multirow[t]{2}{*}{ HLA } & 1 & \multirow[t]{2}{*}{$5.0 \%$} & 17 & 4.6 \\
\hline & & 2 & & 17 & 4.5 \\
\hline \multirow[t]{2}{*}{ HMA 3} & \multirow[t]{2}{*}{ HL3 } & 1 & \multirow[t]{2}{*}{$5.0 \%$} & 155 & 5.3 \\
\hline & & 2 & & 155 & 5.3 \\
\hline \multirow[t]{2}{*}{ SP 1} & \multirow[t]{2}{*}{ - } & 1 & \multirow[t]{2}{*}{$4.5 \%$} & 15 & 5.4 \\
\hline & & 2 & & 18 & 4.8 \\
\hline \multirow[t]{2}{*}{ SP2 } & \multirow[t]{2}{*}{ - } & 1 & \multirow[t]{2}{*}{$4.5 \%$} & 14 & 4.9 \\
\hline & & 2 & & 13 & 5.5 \\
\hline
\end{tabular}


Table 4.15. List of complex modulus test specimens prepared with binder PG 52-34

\begin{tabular}{|c|c|c|c|c|c|}
\hline Mix Type & $\begin{array}{c}\text { Ontario } \\
\text { Designation }\end{array}$ & $\begin{array}{c}\text { Replicate } \\
\#\end{array}$ & $\begin{array}{l}\text { Binder } \\
\text { Content }\end{array}$ & $\begin{array}{c}\text { \# Of Gyrations } \\
\text { Used }\end{array}$ & $\begin{array}{c}\text { Air Voids } \\
(\%)\end{array}$ \\
\hline \multirow[t]{2}{*}{ HMA 1} & \multirow[t]{2}{*}{ HL8 } & 1 & \multirow[t]{2}{*}{$4.5 \%$} & 15 & 5.3 \\
\hline & & 2 & & 15 & 4.8 \\
\hline \multirow[t]{2}{*}{ HMA 2} & \multirow[t]{2}{*}{ HL4 } & 1 & \multirow[t]{2}{*}{$5.0 \%$} & 10 & 3.5 \\
\hline & & 2 & & 10 & 4.8 \\
\hline \multirow[t]{2}{*}{ HMA 3} & \multirow[t]{2}{*}{ HL3 } & 1 & \multirow[t]{2}{*}{$5.0 \%$} & 60 & 6.5 \\
\hline & & 2 & & 75 & 5.2 \\
\hline \multirow[t]{2}{*}{ SP 1} & \multirow[t]{2}{*}{-} & 1 & \multirow[t]{2}{*}{$4.5 \%$} & 30 & 5.03 \\
\hline & & 2 & & 33 & 4.39 \\
\hline \multirow[t]{2}{*}{ SP2 } & \multirow[t]{2}{*}{ - } & 1 & \multirow[t]{2}{*}{$4.5 \%$} & 15 & 5.4 \\
\hline & & 2 & & 15 & 5.0 \\
\hline
\end{tabular}

The physical properties shown in the above tables reflect the nature of the mix as governed by the specifications that assigned a specific function for the mix designations shown earlier in Table 4.9. It is important to notice that in mix HMA 1, which functions as a binder course or a stabilized base, the binder content used was the lowest. Little difference in the physical properties of HMA 2 and HMA 3 was noticed. Mix HMA 2 is usually designed as a binder course. However, in stage construction, layers constructed with such a mix may be exposed to traffic involving direct contact with rotating tires, and hence high binder contents are required. High binder content contributes to durability and flexibility, two important characteristics required in the case of surface layers.

\subsubsection{Mechanistic Characterization of AC Materials}

Parameters selected for the experimental complex modulus investigation were meant to reflect the impact of the state of the material and exposure conditions in the field on the AC response. The selected parameters covered mix types, binder types, compaction quality, curing effect associated with in-service material aging, traffic speed, and 
temperature. The investigated parameters summarized in Table 4.16 are discussed in the following paragraphs.

Table 4.16. Matrix components of AC characterization

\begin{tabular}{|c|c|c|c|c|c|}
\hline \multirow{2}{*}{$\begin{array}{l}\text { Elements of } \\
\text { Investigation }\end{array}$} & \multicolumn{5}{|c|}{ Mix Type } \\
\hline & HMA 1 & HMA 2 & HMA 3 & SP 1 & SP 2 \\
\hline Binder PG & $58-22,52-34$ & $\begin{array}{l}58-22,52- \\
34,64-34\end{array}$ & $\begin{array}{l}58-22,52- \\
34,64-34\end{array}$ & $\begin{array}{l}58-22,52- \\
34\end{array}$ & $\begin{array}{l}58-22,52- \\
34,64-34\end{array}$ \\
\hline $\begin{array}{l}\text { Testing Temperature } \\
\left({ }^{\circ} \mathrm{C}\right)\end{array}$ & $\begin{array}{l}-10,0,20 \\
30,40\end{array}$ & $\begin{array}{l}-10,0,20, \\
30,40\end{array}$ & $\begin{array}{l}-10,0,20, \\
30,40\end{array}$ & $\begin{array}{l}-10,0,20, \\
30,40\end{array}$ & $\begin{array}{l}-10,0,20, \\
30,40\end{array}$ \\
\hline Frequency $(\mathrm{Hz})$ & $\begin{array}{l}20,10,5,1 \\
0.3,0.1\end{array}$ & $\begin{array}{l}20,10,5 \\
1,0.3,0.1\end{array}$ & $\begin{array}{l}20,10,5 \\
1,0.3,0.1\end{array}$ & $\begin{array}{l}20,10,5 \\
1,0.3,0.1\end{array}$ & $\begin{array}{l}20,10,5 \\
1,0.3,0.1\end{array}$ \\
\hline $\begin{array}{c}\text { Curing after } \\
\text { Manufacturing (day) }\end{array}$ & 4 (+/- 1 day) & $\begin{array}{l}4(+/-1 \\
\text { day) }\end{array}$ & $\begin{array}{l}4(+/-1 \\
\text { day })\end{array}$ & $\begin{array}{l}4(+/-1 \\
\text { day) }\end{array}$ & $\begin{array}{l}4(+/-1 \\
\text { day) }\end{array}$ \\
\hline $\begin{array}{c}\text { Air Voids Content } \\
(\%)\end{array}$ & $5+/-0.5$ & $5+/-0.5$ & $5+1-0.5$ & $5+/-0.5$ & $5+/-0.5$ \\
\hline
\end{tabular}

- Mix Types

As mentioned earlier, typical flexible pavements may consist of two or three asphalt concrete sub-layers, including a surface course on top of a binder course. A third layer constructed with an asphalt cement stabilized aggregate base may also be used in some regions. The main feature that differentiates them is the aggregate gradations used to prepare the mix and the percentage of binder content in each sub-layer.

The Marshall mix design procedure was followed to prepare the three mix types simulating all the above AC sub-layers in a flexible pavement, namely HMA 1, HMA 2, and HMA 3. Two mix types of the SuperPave technology were included in the investigation as well. Testing these different $\mathrm{AC}$ mix designs was intended to evaluate the effect of mix types on the mechanistic response and consequently performance. Such an analysis was impossible with the current physical characterization technique. Currently, experience based solely on field observations is relied upon to choose the binder to use in the different applications. Analysis based on complex modulus test results will provide 
the basis for evaluating the effectiveness of binder/aggregate (mix designs) combinations in producing the desirable composite material that meets performance expectations.

\section{- Temperature Condition}

As discussed earlier in Chapter 3, characteristics of asphalt concrete materials are temperature sensitive, where higher temperatures $\left(25^{\circ} \mathrm{C}\right.$ and +$)$ result in a low stiffness, making the road more susceptible to permanent deformations. Meanwhile, low temperatures (below $10^{\circ} \mathrm{C}$ ) result in a brittle $\mathrm{AC}$ layer, making the road susceptible to cracking.

Asphalt cement is behind the temperature sensitivity of the AC mix. By the selection of different performance grade binders and a wide range of test temperatures, the temperature sensitivity of the mix has been adequately addressed in this thesis.

The adopted temperature range $\left(-10,0,20,30\right.$, and $\left.40^{\circ} \mathrm{C}\right)$ covers the impact of seasonal variations in Canada. However, characteristics of AC materials in regions exposed to temperatures outside the adopted range can be extrapolated from the master curves constructed using results of tests conducted in this study (see Master Curve discussed in Chapter 3 along with results and analysis discussed in Chapter 6).

\section{- Traffic Characteristics}

The repetitive nature of traffic loading, which creates the sinusoidal response discussed in Section 3.2, was simulated in the laboratory using the MTS hydraulic loading system. Traffic speeds have a significant impact on the response of AC materials. As a result of the viscous nature of $\mathrm{AC}$ materials, lower speeds usually result in high deformations compared with the high speeds that prevail on highways. Hence, a wide range of 
vehicular speeds have been included in the study, focusing on the lower range because of their critical impact on permanent deformations.

The combination of test temperatures and loading frequencies covers well the critical conditions in urban streets, offering an excellent opportunity to address the severity of damage observed in this essential component of the Canadian roadway network.

\section{- Binder Types}

The binder characterization technique (PG Grade) proved unsuccessful in discriminating between commercially available products. The complex modulus test offers an excellent opportunity to deliver the required evaluation. Roads are not paved with binders and hence, attempts to evaluate them independently from the mix were not successful. The interaction between the binder and mineral aggregates is a complicated matter involving adhesion inhibiters associated with chemical reaction, adsorption, and interfacial energy. It also involves physical interaction related to aggregate wetting and film thickness. The complex modulus test delivers a quick approach to achieving a quantifiable property that may indicate the appropriateness of selecting a binder among available options. Two conventional binder types (neat binders) were selected to examine the effect of using these different binder grades on the characteristic response of the AC mix. Neat binders used in this research included PG 58-22 and PG 52-34. The resulting trend was then compared with the response from a mix with an engineered binder (PG 64-34).

\section{- Construction Quality}

Construction quality is a major concern among transportation authorities. Many jurisdictions use either density or the percent of air voids content to evaluate construction 
quality. However, limits specified by each jurisdiction may differ, where some specifications recommend a minimum air voids content of 3.5 to $4.5 \%$ as is the case with MTO specifications [71]. The minimum value is introduced to allow for free flow of binders into the voids and prevent bleeding in case of shear flow under the action of traffic loading. Ohio State DOT specified a range of 3.5 to $5.0 \%$ [74], while Saskatchewan DOT specifications include an air voids requirement between 4 and $9 \%$ $[75]$.

Moreover, air void percents higher than the specified were recorded. Contractors barely achieve the desired quality of construction represented by air voids. In some circumstances, air voids achieved approached $11 \%$, with contractors being asked to pay a penalty. In the absence of a mechanistic mix characterization technique and a model needed to predict potential damage, the formulae used to calculate penalties are not sound or easy to defend.

Civil engineers have long recognized that the microstructure of a material like AC, such as air voids and particle distribution and orientation, is influenced by compaction. Mineral aggregates reorient in response to the applied compaction effort. From this discussion, it can be drawn that quality control is a significant factor that will affect $\mathrm{AC}$ response, and hence the performance. Table 4.17 shows the SuperPave mix specimens prepared using binder PG 64-34 at varying compaction effort resulting in different air voids percentages. These samples were tested using the developed complex modulus test protocol to examine the effect of quality control on $\mathrm{AC}$ mechanistic properties. The results pertaining to these specimens are shown later in Chapter 6. 
Table 4.17. SuperPave mix specimens prepared under different compaction effort

\begin{tabular}{|c|c|c|}
\hline Sample ID & \# Of Gyrations & Air Voids \% \\
\hline SP2-64-34-1 & 3 & 10.89 \\
\hline SP2-64-34-2 & 5 & 10.12 \\
\hline SP2-64-34-3 & 8 & 9.95 \\
\hline SP2-64-34-4 & 13 & 7.14 \\
\hline SP2-64-34-5 & 19 & 5.70 \\
\hline SP2-64-34-5 & 40 & 3.84 \\
\hline SP2-64-34-6 & 50 & 3.49 \\
\hline
\end{tabular}

\section{- Effect of Curing}

The majority of construction materials experience strength gain with time, a phenomenon referred to as curing. The curing specification for Portland cement concrete (PCC) involves strength tests after 28 days. However, the situation is more complicated in the case of $\mathrm{AC}$ mixtures in which the period needed to arrive at the maximum strength depends on many factors. These include the nature of the crude oil used in producing the binder and environmental conditions such as temperature, moisture and wind. The curing process is also influenced by the thickness of the constructed layer. There are no definitive curing specifications beyond recommending a mat temperature above which the road should not be exposed to traffic to avoid loading it at a vulnerable state of low stiffness. Current specifications, such as for Marshall stability, recommend performing the mechanical test for stability within 24 hours of manufacturing the sample. However, in actual practice in the field, the $\mathrm{AC}$ layer is exposed to loading shortly after construction, such as in the case involving an overlay on an urban road. Urban transportation authorities are forced to open a newly repaired road to traffic to avoid congestion. Such loading circumstances are expected to cause premature damage in the surface layer of flexible pavements because of the low stiffness of the material. 
To investigate the influence of aging on the stiffness of $\mathrm{AC}$ mixtures, specimens were prepared at almost the same air voids and tested at different ages. Specimens were tested just 18 hours after preparation to represent the case of an urban road under maintenance, and others were tested 6,16 and 30 days after preparation. A detailed analysis of complex modulus tests performed on these specimens is reported in Chapter 6.

\subsection{Implementations of Complex Modulus Test Output}

Upon the determination of the viscoelastic properties of different mixes prepared with different binders and tested under different test conditions, the reduced test data were tested for implementation in two major applications. The first application involves using the produced data as input to analytical models. The second application relates to attempts by the industry to introduce a scheme that will enable analytical model users to perform an analysis without the need to conduct the complex modulus test. This second application involves the use of models to predict the complex modulus using physical characteristics produced by simple and commonly used tests performed on the mix and its components. These two types of applications are highlighted in the following paragraphs.

\subsubsection{Analytical Models}

Viscoelastic parameters are compatible with analytical model applications and will facilitate design and analysis exercises looking into material and construction-related variables. Rheological models capable of predicting AC characteristic response to external stimuli could be incorporated into these models to trace the behaviour of the material as part of the road structure. The Huet-Sayegh model is considered the best of several rheological models for its close representation of the actual behaviour of $\mathrm{AC}$ 
materials. This model was incorporated into an analytical model developed at NRC Canada to estimate the contribution of the $\mathrm{AC}$ layer to the overall performance of a road structure. Utilizing the calculated dynamic moduli and phase angles of a certain mixture, the eight parameters of the Huet-Sayegh model mentioned in Chapter 3 were determined. The AC characteristics for different mixes were used to populate a material library including commonly used $\mathrm{AC}$ mixes together with unbound materials, such as cohesive and non-cohesive soils. The NRC model picks up the eight parameters from the library to estimate the contribution of the AC layer constructed with a certain mix to the overall permanent deformation of a road.

\subsubsection{AASHTO 2002 Design Guide}

In the case of other model formats, such as the new ASSHTO 2002 Design Guide, the complex modulus test provides the mechanistic input data needed to run the software. ASSHTO 2002 Guide requires the dynamic modulus parameter to be determined at specific temperatures and loading frequencies, all included in the ranges established in this experimental program [60].

Results of attempts made to apply the outcomes of the proposed characterization technique in analytical modeling, focusing on AASHTO Level 1 design, are discussed in Chapter 7.

\subsubsection{Predictive Equations}

To evaluate the validity of the two equations developed as part of the 2002 Design Guide discussed in Chapter 3, an experimental investigation was developed to evaluate the validity of the proposed predictive equations purposed as a replacement for conducting 
the complex modulus test. The data gathered from a number of tests performed on a variety of $\mathrm{AC}$ mixes were tabulated in a database, which represents part of the material library built to facilitate the implementation of Level 3 of the ASSHTO 2002 Design Guide by road agencies that do not have testing capabilities. The library covers different aggregate gradations, binder types, and mix designs.

The test was generated using the complex modulus testing protocol based on a straincontrolled mechanical test in which a sinusoidal axial strain (tension and compression) was applied at a given temperature and loading frequency. The resulting axial stress response of the specimen was measured and used together with the applied strain to calculate the dynamic modulus, and then the phase angle.

Materials used for assessment of the predictive equations included the two mix designs (Marshall and SuperPave) discussed in Chapter 5.

Results of physical characterization of these mixtures were needed in this study including properties from the mix design exercise and others from other dedicated tests. The required properties include:

- Binder content by total mass of mixture $\left(\mathrm{P}_{\mathrm{b}}\right)$

- Maximum specific gravity of mixture $\left(\mathrm{G}_{\mathrm{mm}}\right)$

- Bulk specific gravity of compacted mixture $\left(\mathrm{G}_{\mathrm{mb}}\right)$

- Voids in mineral aggregate as a percent of bulk volume (VMA)

- Air voids in compacted mixture as a percent of total volume $\left(\mathrm{V}_{\mathrm{a}}\right)$

- Effective bitumen content, as percent by volume $\left(\mathrm{V}_{\text {beff }}\right)$ 
Specimens from the mixes included in this investigation were prepared using a binder content of $5 \pm 0.5 \%$. The binder content was kept constant to reduce the number of variables considered in the predictive equation investigation.

Table 4.18. Physical properties of HMA 3 mix

\begin{tabular}{|c|c|c|c|}
\hline \multirow{2}{*}{ Property } & \multicolumn{3}{|c|}{ Specimen ID } \\
\cline { 2 - 4 } & HMA 3 58-22 & HMA 3 64-34 & HMA 3 52-34 \\
\hline $\mathrm{P}_{\mathrm{b}}(\%)$ & 5 & 5 & 4.9 \\
\hline $\mathrm{G}_{\mathrm{mm}}$ & 2.530 & 2.477 & 2.503 \\
\hline $\mathrm{G}_{\mathrm{mb}}$ & 2.396 & 2.337 & 2.375 \\
\hline $\mathrm{G}_{\mathrm{sb}}$ & 2.720 & 2.720 & 2.720 \\
\hline $\mathrm{VMA}$ & 16.3 & 18.4 & 17.0 \\
\hline $\mathrm{V}_{\mathrm{a}}$ & 5.3 & 5.7 & 5.1 \\
\hline $\mathrm{V}_{\text {beff }}$ & 11.0 & 12.7 & 11.9 \\
\hline
\end{tabular}

Table 4.19. Physical properties of HMA 2 mix

\begin{tabular}{|c|c|c|c|}
\hline \multirow{2}{*}{ Property } & \multicolumn{3}{|c|}{ Specimen ID } \\
\cline { 2 - 4 } & HMA 2 - PG 58-22 & HMA 2 - PG 64-34 & HMA 2 - PG 52-34 \\
\hline $\mathrm{P}_{\mathrm{b}}(\%)$ & 5 & 5.1 & 5 \\
\hline $\mathrm{G}_{\mathrm{mm}}$ & 2.536 & 2.506 & 2.524 \\
\hline $\mathrm{G}_{\mathrm{mb}}$ & 2.421 & 2.389 & 2.402 \\
\hline $\mathrm{G}_{\mathrm{sb}}$ & 2.742 & 2.742 & 2.742 \\
\hline $\mathrm{VMA}$ & 16.1 & 17.3 & 16.8 \\
\hline $\mathrm{V}_{\mathrm{a}}$ & 4.5 & 4.7 & 4.8 \\
\hline $\mathrm{V}_{\text {beff }}$ & 11.6 & 12.7 & 12.0 \\
\hline
\end{tabular}

Table 4.20. Physical properties of HMA 1 mix

\begin{tabular}{|c|c|c|}
\hline \multirow{2}{*}{ Property } & \multicolumn{2}{|c|}{ Specimen ID } \\
\cline { 2 - 3 } & HMA 1 - PG 58-22 & HMA 1 - PG 52-34 \\
\hline $\mathrm{P}_{\mathrm{b}}(\%)$ & 4.5 & 4.5 \\
\hline $\mathrm{G}_{\mathrm{mm}}$ & 2.540 & 2.566 \\
\hline $\mathrm{G}_{\mathrm{mb}}$ & 2.412 & 2.429 \\
\hline $\mathrm{G}_{\mathrm{sb}}$ & 2.740 & 2.740 \\
\hline $\mathrm{VMA}$ & 15.9 & 15.3 \\
\hline $\mathrm{V}_{\mathrm{a}}$ & 5.0 & 5.3 \\
\hline $\mathrm{V}_{\text {beff }}$ & 10.9 & 10.0 \\
\hline
\end{tabular}


Table 4.21. Physical properties of SP $1 \mathrm{mix}$

\begin{tabular}{|c|c|c|}
\hline \multirow{2}{*}{ Property } & \multicolumn{2}{|c|}{ Specimen ID } \\
\cline { 2 - 3 } & SP1- PG 58-22 & SP1 - PG 52-34 \\
\hline $\mathrm{P}_{\mathrm{b}}(\%)$ & 4.5 & 4.5 \\
\hline $\mathrm{G}_{\mathrm{mm}}$ & 2.544 & 2.547 \\
\hline $\mathrm{G}_{\mathrm{mb}}$ & 2.415 & 2.418 \\
\hline $\mathrm{G}_{\mathrm{sb}}$ & 2.721 & 2.721 \\
\hline $\mathrm{VMA}_{\mathrm{y}}$ & 15.2 & 15.1 \\
\hline $\mathrm{V}_{\mathrm{a}}$ & 5.1 & 5.1 \\
\hline $\mathrm{V}_{\text {beff }}$ & 10.2 & 10.1 \\
\hline
\end{tabular}

Table 4.22. Physical properties of SP 2 mix

\begin{tabular}{|c|c|c|c|}
\hline \multirow{2}{*}{ Property } & \multicolumn{3}{|c|}{ Specimen ID } \\
\cline { 2 - 4 } & SP2 - PG 58-22 & SP2 - PG 64-34 & SP2 - PG 52-34 \\
\hline $\mathrm{P}_{\mathrm{b}}(\%)$ & 4.5 & 4.5 & 4.5 \\
\hline $\mathrm{G}_{\mathrm{mm}}$ & 2.577 & 2.590 & 2.570 \\
\hline $\mathrm{G}_{\mathrm{mb}}$ & 2.434 & 2.435 & 2.441 \\
\hline $\mathrm{G}_{\mathrm{sb}}$ & 2.755 & 2.755 & 2.755 \\
\hline $\mathrm{VMA}$ & 15.6 & 15.6 & 15.4 \\
\hline $\mathrm{V}_{\mathrm{a}}$ & 5.6 & 6.0 & 5.0 \\
\hline $\mathrm{V}_{\mathrm{beff}}$ & 10.1 & 9.6 & 10.4 \\
\hline
\end{tabular}

$$
\begin{aligned}
& \log \left|E^{*}\right|=-1.249937+0.029232 P_{200}-0.001767\left(P_{200}\right)^{2}+0.002841 P_{4}-0.058097 V_{a} \\
& -0.802208 \frac{V_{b e f f}}{\left(V_{b e f f}+V_{a}\right)}+\frac{\left[3.871977-0.0021 P_{4}+0.00395 P_{38}-0.000017\left(P_{38}\right)^{2}+0.00547 P_{34}\right]}{1+e^{(-0.603313-0.313351 \log f-0.393532 \log \eta)}}
\end{aligned}
$$

\subsection{Summary}

The laboratory experimental investigation discussed above covers three major components of this thesis. These components included development of the complex modulus test, characterization of common AC mixes, and implementation of this characterization technique in pavement design and analysis. The results of these three investigations are presented as follows: 
- Results of test development are presented and discussed in Chapter 5 to establish the complex modulus test protocol.

- Application of the developed complex modulus testing technique for characterizing common $\mathrm{AC}$ mixes included in this experimental program is discussed in Chapter 6. The material library populated with the determined properties of these mixes is discussed in Chapter 7.

- The complex modulus test data will be used in the implementation of the new characterization technique in Chapter 7 . The design and analysis of pavement structures using analytical models, and the validation of predictive equations will be discussed using the material library. 


\section{CHAPTER 5 \\ DEVELOPMENT OF TEST PROTOCOL FOR COMPLEX MODULUS}

\subsection{Introduction}

This chapter discusses the results of tests performed as part of the process to support the conceptualization and refining of the complex modulus test procedure. The objective was to establish a test protocol that could be used effectively in laboratories to mechanistically characterize asphalt concrete response based on the complex modulus approach. The pilot tests described in Section 4.2 of Chapter 4 were used to investigate a number of critical test components before finalizing the test procedure.

The proposed test protocol involved a number of factors that influence the response of AC materials, and hence, the complex modulus parameters such as temperature, loading mode, strain magnitude, loading frequency, test set-up, data acquisition system, and data reduction techniques. Relevant results from the pilot investigation performed to support the development of the test protocol are discussed in this chapter.

\subsection{Loading Mode}

As discussed in Chapter 4, the cyclic tension-compression loading mode was selected to perform the complex modulus test. In order to maintain the characterization technique of AC materials within the linear viscoelastic response, a strain-controlled mode was chosen for this study. A review of current attempts being made by researchers [73] revealed that, depending on the mix type, stress-controlled tests might result in high strain levels leading to a non-linear response. The switch into a non-linear response may take place without the knowledge of the operator, especially when permanent deformations are acquired at a relatively low stress magnitude (lower than tire pressure associated with 
commercial vehicles). The typical stress-strain relationship established for a SuperPave mix (SP 2, binder PG 52-34) tested at $25^{\circ} \mathrm{C}$ and a frequency of $20 \mathrm{~Hz}$ is shown in Figure 5.1. The plotted relationship suggests that a transition to a non-linear response took place at a stress of $200 \mathrm{kPa}$ and a strain magnitude of $100 \mu \varepsilon$. The selected strain magnitude is discussed in more detail in the following subsection.

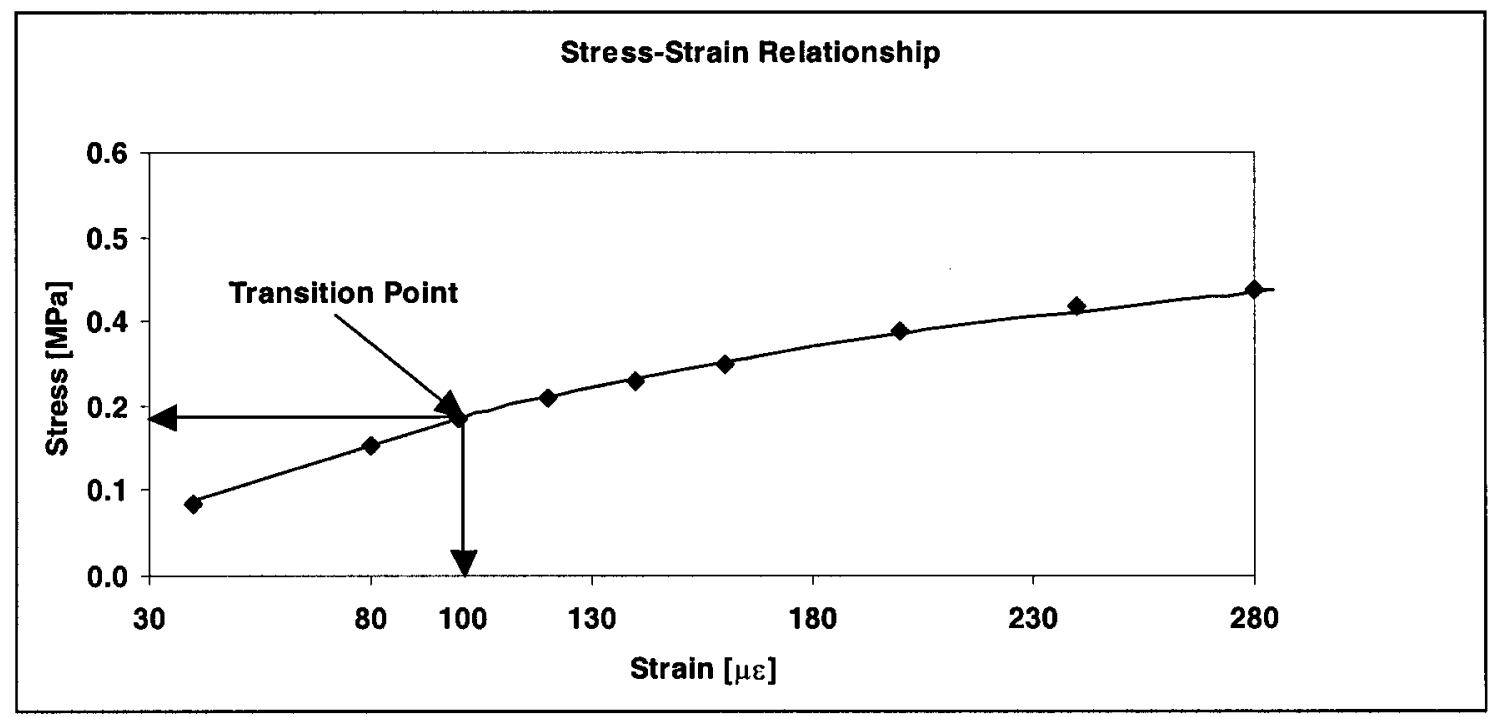

\section{Figure 5.1. Typical stress-strain relationship established at $25^{\circ} \mathrm{C}$ and a loading frequency of $20 \mathrm{~Hz}$}

\subsection{Strain Magnitude}

Even though AC mixes do not exhibit ideal linear viscoelastic behaviour because of the heterogeneity of the mix, an approximation of such behaviour is generally accepted at low strain levels. In order to investigate the limits where linearity of the response is experienced in common $\mathrm{AC}$ mixes, the test designed for the linearity check in Chapter 4 was performed using an HMA 2 mix prepared with a PG 64-34 binder. The intention was to determine displacement magnitudes that could be applied safely within the linear state. AC properties obtained at two temperatures $\left(+10\right.$ and $\left.+25^{\circ} \mathrm{C}\right)$ were used to demonstrate 
the process followed in the study to determine the point where linearity of the response prevails.

The linearity check was performed by analyzing the stresses and the corresponding axial strains obtained at different frequencies. Results from tests conducted at $+10^{\circ} \mathrm{C}$, shown in Figure 5.2, revealed that linearity prevailed with the increase in strain magnitudes until a strain value of $240 \mu \varepsilon$. The stress dropped slightly for the sample tested at $20 \mathrm{~Hz}$ using a strain magnitude of $240 \mu \varepsilon$ which may suggest potential damage. Results of the linearity check performed using the stress-strain relationship was supported by the plot of dynamic modulus against strain values measured at $+10^{\circ} \mathrm{C}$ for different loading frequencies. Results showed that a reasonable linear behaviour may be assumed for strain values lower than or equal to $120 \mu \varepsilon$ as shown in Figure 5.3.

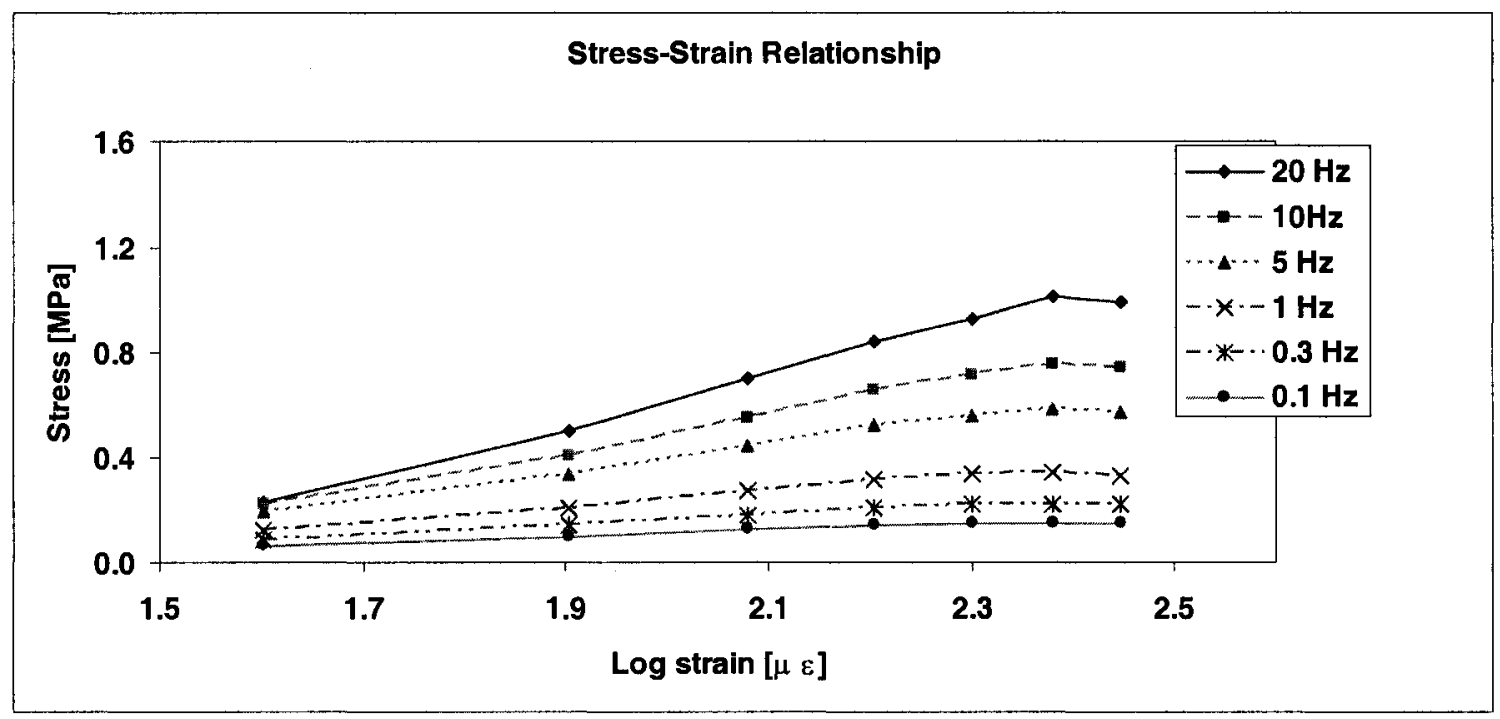

Figure 5.2. Typical results of linearity check based on $(\sigma-\varepsilon)$ relationship $\left[+10^{\circ} \mathrm{C}\right]$ 


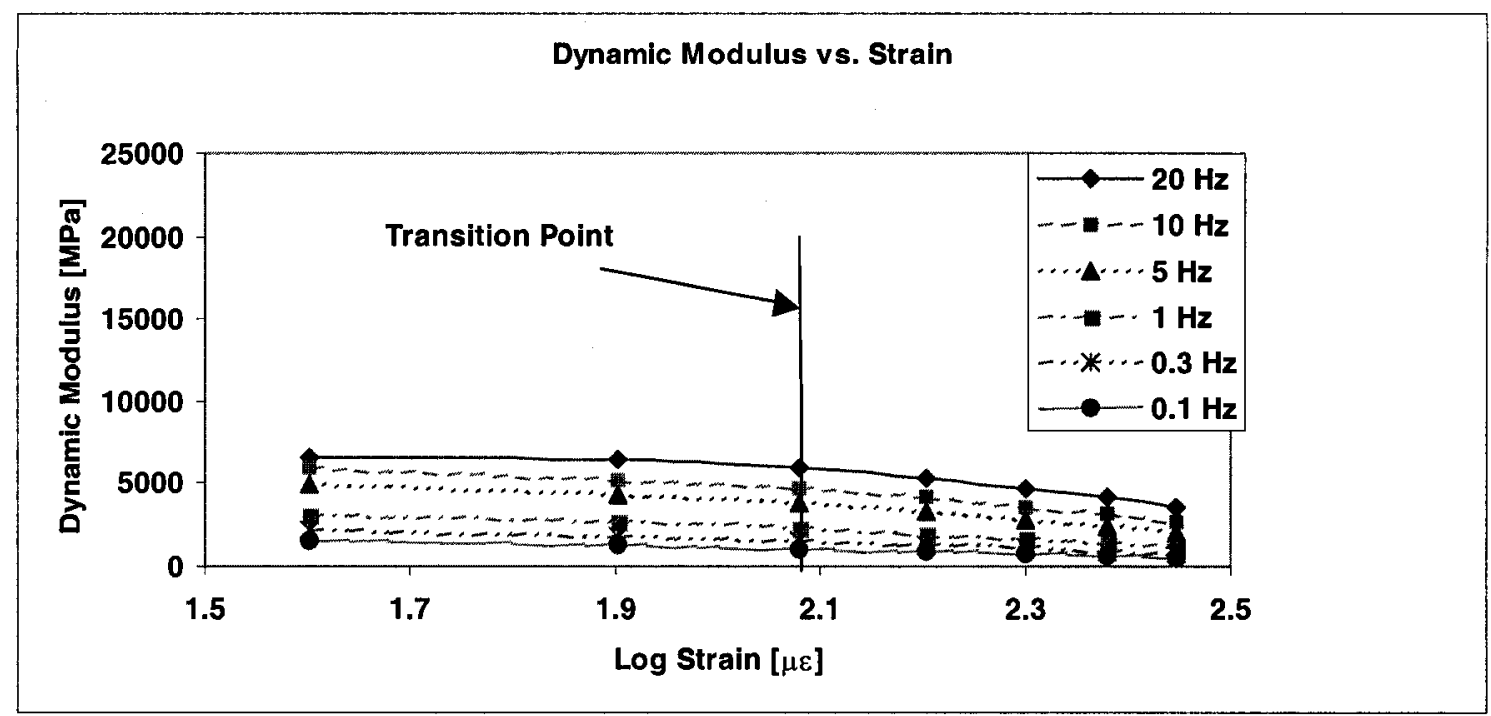

Figure 5.3. Typical results of linearity check using the dynamic modulus $\left[+10^{\circ} \mathrm{C}\right]$

Linearity was checked at relatively warmer temperature $\left(+25^{\circ} \mathrm{C}\right)$. The stress-strain relationship was plotted in Figure 5.4 to show the relationship between the dynamic modulus and strain (see Figure 5.5.) Although no transition from linearity to non linearity was apparent in the stress-strain relationship for tests performed at $+25^{\circ} \mathrm{C}$, the dynamic modulus-strain relationship shown in Figure 5.5 reflected such transition at a strain magnitude of $100 \mu \varepsilon$.

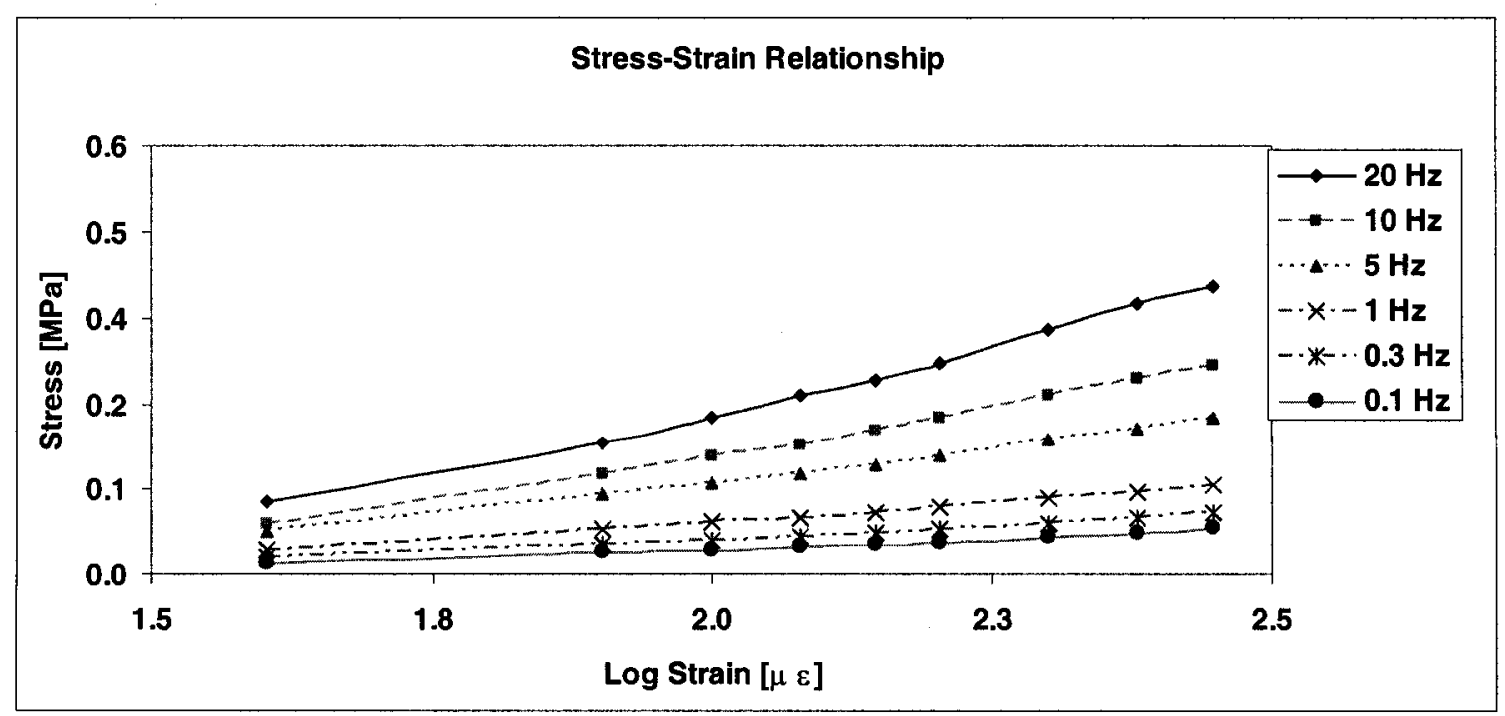

Figure 5.4. Typical results of linearity check based on $(\sigma-\varepsilon)$ relationship $\left[+25^{\circ} \mathrm{C}\right]$ 


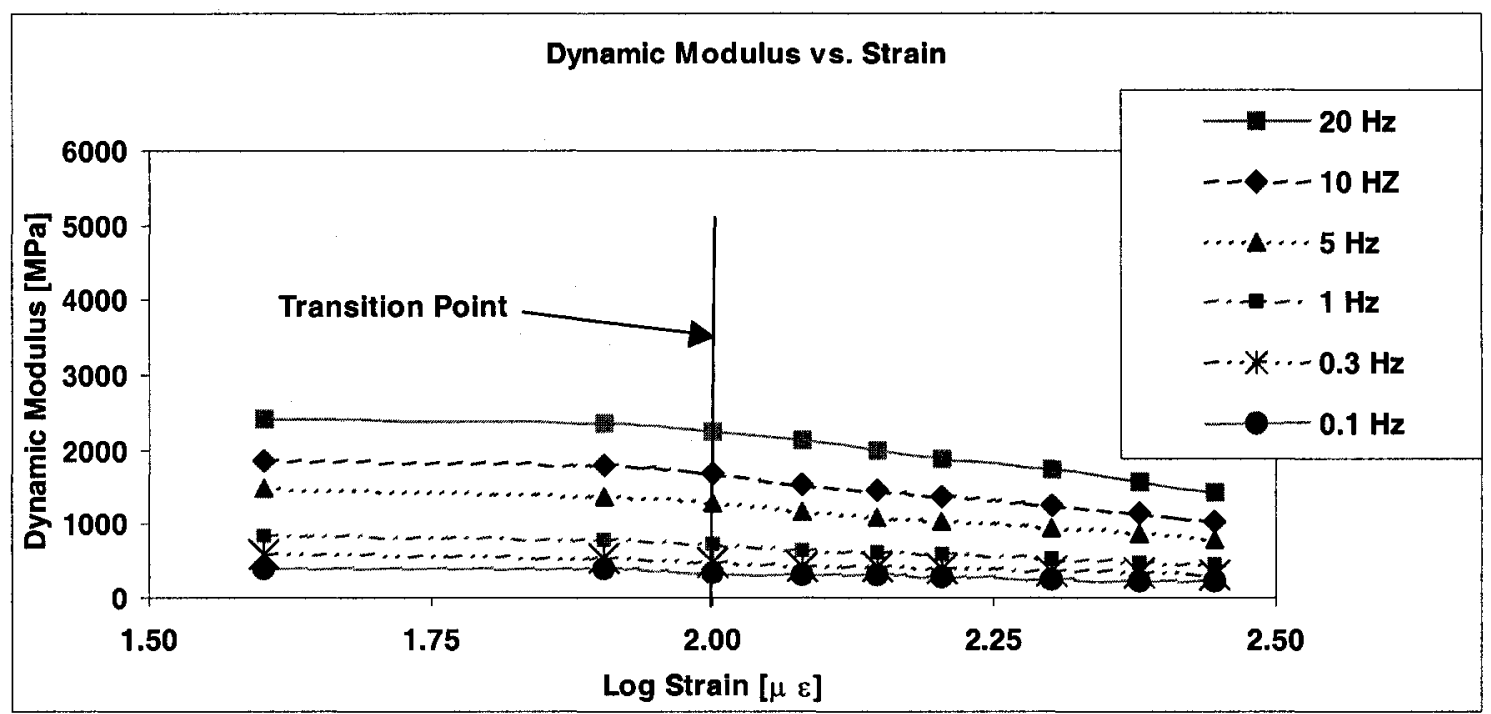

Figure 5.5. Typical results of linearity check using the dynamic modulus $\left[+25^{\circ} \mathrm{C}\right]$

Selection of the strain amplitude was originally made to conform with the need to maintain the mechanical test within the linear viscoelastic state. Meanwhile, very low strain levels were avoided to eliminate the potential for energy loss within the equipment at contact points and connections of the test set-up. The loss of energy could affect the clarity of signals that represent the material response. Accordingly, a strain amplitude of $100 \mu \varepsilon$ was selected for the complex modulus testing program established for this study. It worth mentioning that the linearity checks were performed routinely on all mixes, which reflected patterns similar to those discussed in the typical examples displayed above.

\subsection{Low Temperature Behaviour:}

With the $\mathrm{AC}$ behaviour in warm temperatures examined earlier (checking the linearity at +10 and $+25^{\circ} \mathrm{C}$ ), other tests were conducted to examine low temperature behaviour. Table 5.1 shows results of test performed at $-10^{\circ} \mathrm{C}$ using a loading frequency of $10 \mathrm{~Hz}$. 
The determined dynamic moduli and phase angles were used to calculate the storage and loss moduli based on Equations 3.12 and 3.13.

Table 5.1. Results of test performed at $-10^{\circ} \mathrm{C}$ using a loading frequency of $10 \mathrm{~Hz}$ to check low temperature behaviour

\begin{tabular}{|c|c|c|c|c|c|c|c|}
\hline $\begin{array}{c}\text { Strain } \\
{[\mu \varepsilon]}\end{array}$ & $\begin{array}{c}\text { Stress } \\
{[\mathrm{MPa}]}\end{array}$ & $\begin{array}{c}\left|E^{*}\right|, \\
{[\mathrm{MPa}]}\end{array}$ & $\begin{array}{c}\text { Phase } \\
\text { Angle }\left[{ }^{\circ}\right]\end{array}$ & $\begin{array}{c}\mathrm{E}_{1}, \\
{[\mathrm{MPa}]}\end{array}$ & $\begin{array}{c}\mathrm{E}_{2}, \\
{[\mathrm{MPa}]}\end{array}$ & $\begin{array}{c}\mathrm{E}_{1}:\left|E^{*}\right| \\
\%\end{array}$ & $\mathrm{E}_{2} / \mathrm{E}_{1}$ \\
\hline 67 & 2.195 & 32800 & 4.5 & 32699 & 2573 & 99.70 & 0.079 \\
\hline 90 & 2.815 & 31300 & 4.2 & 31216 & 2292 & 99.70 & 0.073 \\
\hline 120 & 3.454 & 28800 & 5.6 & 28663 & 2810 & 99.50 & 0.098 \\
\hline
\end{tabular}

From Table 5.1, values of the storage modulus $\mathrm{E}_{1}$ constituted over $99 \%$ of the dynamic modulus values suggesting an elastic behaviour. This observation is substantiated by the extremely small values of the phase angle determined in the tests (within the range of measuring error). The stress-strain and the dynamic modulus-strain relationships established from these tests are shown in Figure 5.6. This figure reflects clearly the linear elastic response at a low temperature of $-10^{\circ} \mathrm{C}$. The Young modulus calculated from Figure 5.6 which equal to ( $30765 \mathrm{MPa}$ ) is very close to the average value of the dynamic modulus shown in Table 5.1.
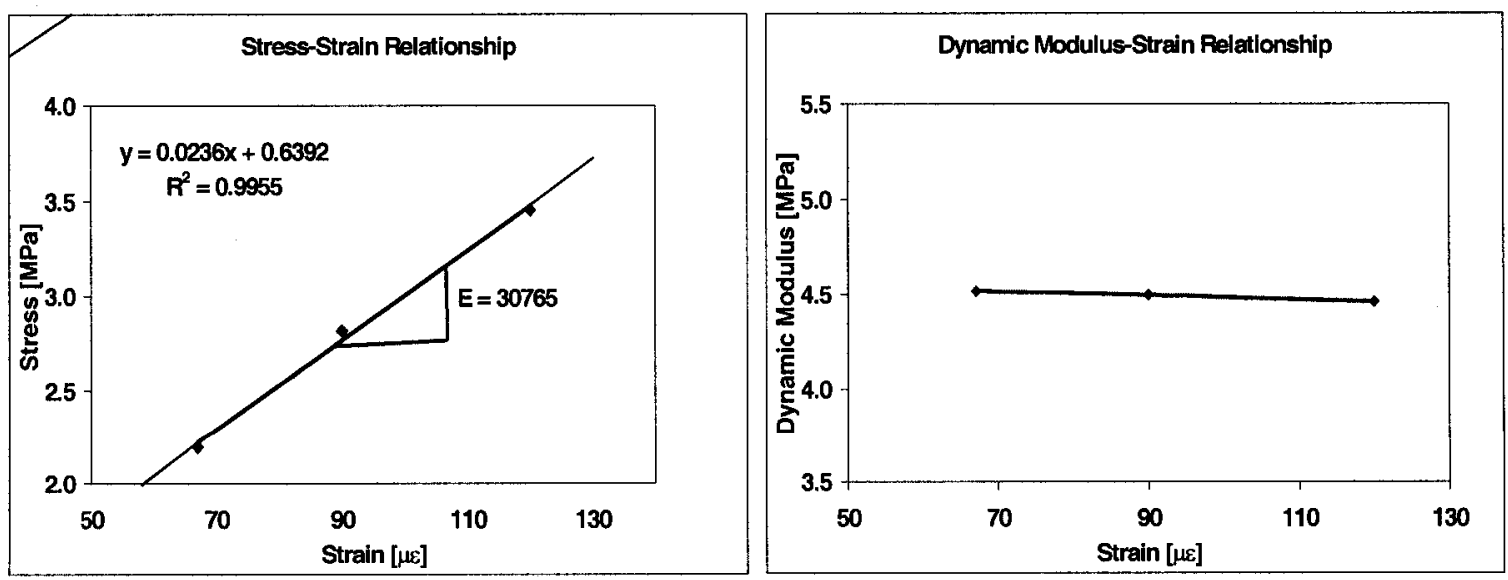

Figure 5.6. (a) Stress-strain relationship, (b) Dynamic modulus-strain relationship for test performed at $-10^{\circ} \mathrm{C}$ 
Recalling Equation 3.10 (reproduced below), which relates the complex modulus to the elastic and viscous moduli components, the results of the above experimental investigation seem to be in agreement with the analytical approach;

$E^{*}=\mathrm{E}_{1}+\mathrm{i} \mathrm{E}_{2}$

where $E_{1}$ is the storage (elastic) modulus

and $E_{2}$, is the loss (viscous) modulus,

From Equation 3.10, the norm of complex modulus which is the dynamic modulus can be written as shown in Equation 5.1 which can be written as Equation 5.2

$\left|E^{*}\right|=\sqrt{E_{1}^{2}+E_{2}^{2}}$

$\left|E^{*}\right|=\mathrm{E}_{1} \times \sqrt{1+\left(\frac{\mathrm{E}_{2}}{\mathrm{E}_{1}}\right)^{2}}$

At relatively low temperatures such as the $-10^{\circ} \mathrm{C}$ used in this test, the ratio $\mathrm{E}_{2} / \mathrm{E}_{1}$ becomes very small as depicted in Table 5.1 Accordingly, $\left|E^{*}\right|$ value approaches $\mathrm{E}_{1}$ as shown in Equation 5.2. In other words, the elastic component dominates the behaviour at low temperatures suggesting an elastic response. Such behaviour can be represented graphically as shown in Figure 5.7.

\subsection{Loading Frequency}

Attempts were made to extend the loading frequency range to incorporate higher and lower frequencies ( $>20$ and $<0.1 \mathrm{~Hz}$ respectively). However, as discussed in Chapter 4 , as a result of safety concerns associated with performing tests at extreme frequencies, such frequencies were eliminated from the final test protocol. Complex modulus tests were 
performed in a frequency range including $20,10,5,1,0.3$, and $0.1 \mathrm{~Hz}$, in decreasing order for the reasons reported in Chapter 3.

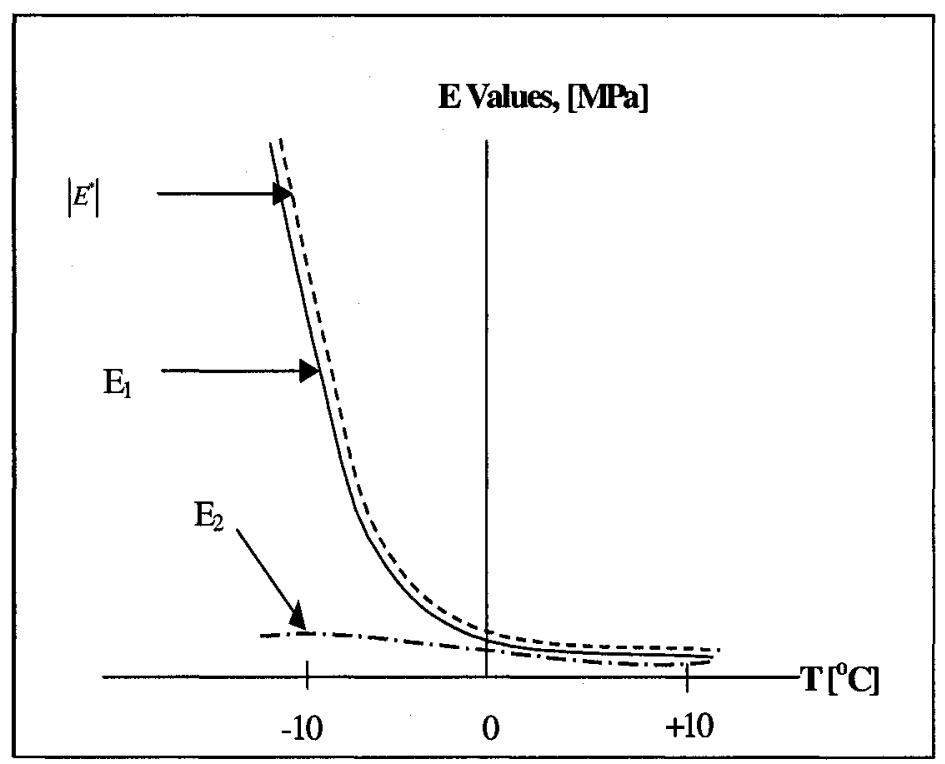

Figure 5.7. Behaviour of AC materials at low temperatures

\subsection{Temperature Conditioning}

Attempts were made to extend the testing temperatures on both sides of the temperature range: cold and warm. Trials made at $-20^{\circ} \mathrm{C}$ were unsuccessful owing to breakage of specimens during the test as discussed in Chapter 4. Figure 5.8 shows results of an unsuccessful attempt for testing at $-20^{\circ} \mathrm{C}$ performed using an HMA 2 mix prepared with a PG 58-22 binder at a loading frequency of $20 \mathrm{~Hz}$. The sample shown in Figure 5.9 failed (broke) after applications of 50 load cycles or less. Failure occurred close to the loading plate/sample interface. The majority of the failure surface was located within the binder that coated aggregates and filled gaps in the aggregate skeleton, with the exception of a limited number of broken aggregates as shown in Figure 5.9. This observation suggests the potential of adhesion failure taking place within the area adjacent to the loading plate. 
Tests performed at $-10^{\circ} \mathrm{C}$ were successful and the selected strain amplitudes were reached without specimen breakage.

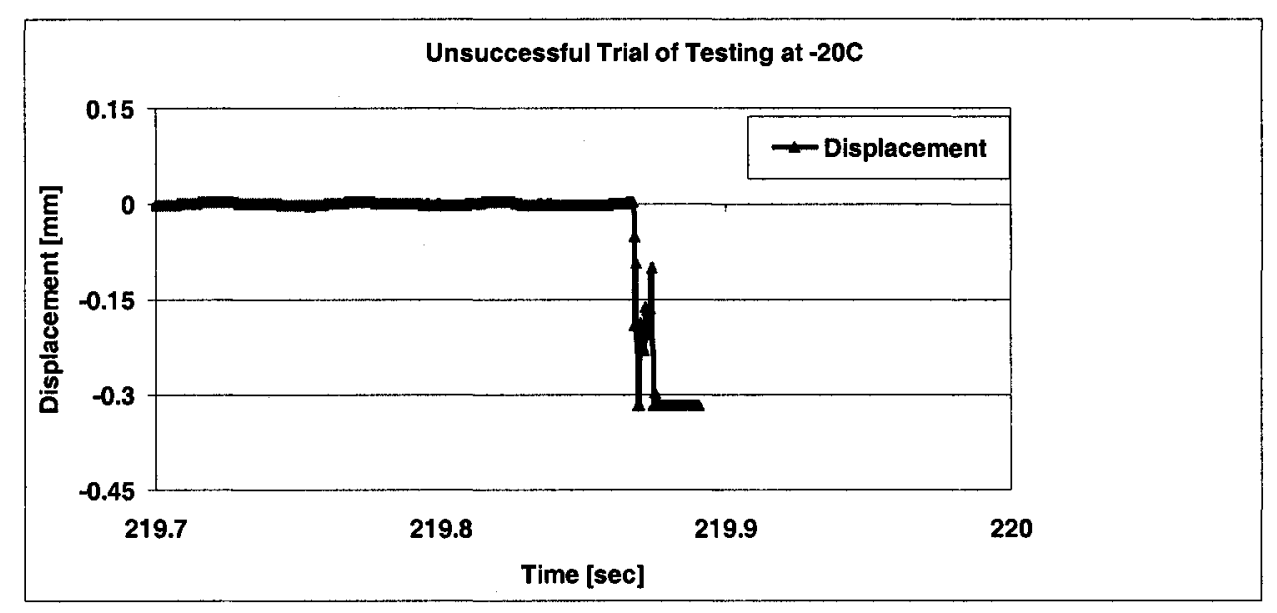

Figure 5.8. Trial of testing at $-20^{\circ} \mathrm{C}, 20 \mathrm{~Hz}$

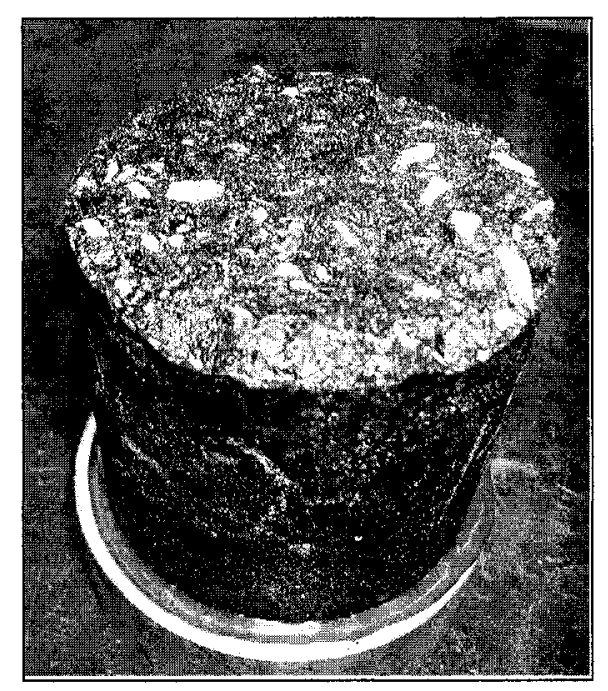

Figure 5.9. Sample broken during a test performed at $-20^{\circ} \mathrm{C}, 20 \mathrm{~Hz}$.

On the other hand, preliminary results of attempts to perform tests at $+50^{\circ} \mathrm{C}$ revealed nonlinear behaviour even at low strain amplitude, where permanent deformation accumulated in all test frequencies. Figure 5.10 shows results of an attempt made to perform a test at $+50^{\circ} \mathrm{C}$ using a SuperPave mix (SP 1) prepared with a PG 52-34 binder. Loading frequencies used were $20,10,5$, and $1 \mathrm{~Hz}$. The test was started using a loading frequency of $20 \mathrm{~Hz}$ with a zero $\mathrm{mm}$ displacement reading at the beginning of data collection. 
However, the displacement recorded at the beginning of data collection at $1 \mathrm{~Hz}$ was 0.045 mm. A similar shift was observed in a number of other samples across the whole frequency sweeps, which is an indication of the accumulation of permanent deformations during these tests performed at $+50^{\circ} \mathrm{C}$.

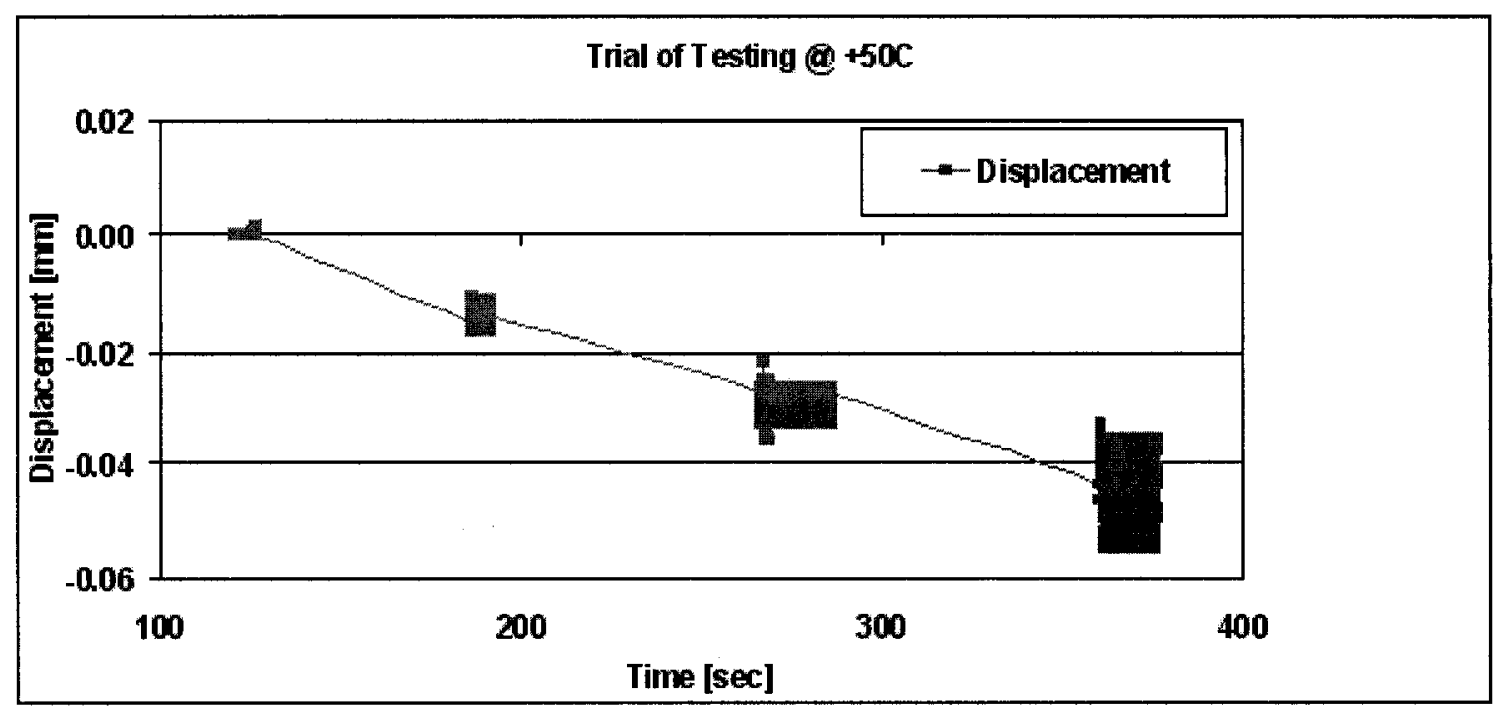

\section{Figure 5.10. Results of trial test at $+50^{\circ} \mathrm{C}$ showing tendency of permanent deformation}

The decision in this thesis to limit $\mathrm{AC}$ complex modulus measurements within the linear viscoelastic state led to the selection of a temperature conditioning regime covering -10 , $0,20,30$, and $40^{\circ} \mathrm{C}$. The time period required for conditioning the specimens at the desired temperature was determined using a trial and error approach involving tests on a number of $\mathrm{AC}$ specimens with a thermocouple fixed inside. Results revealed that a minimum period of 1.5 hours is sufficient for adjusting the sample temperature when proceeding from one temperature to another within the selected range (e.g., from -10 to 0 , 0 to 20,20 to 30 , and 30 to $40^{\circ} \mathrm{C}$ ). 


\subsection{Test Set-up}

The MTS-810 testing system offered the necessary flexibility for conducting the complex modulus test developed in this thesis. However, a number of trials were made to select a suitable arrangement of the test components attached to the loading frame to adequately perform the test as discussed below.

- Load Cell Position. Initially, the load cell was placed in contact with the moving hydraulic actuator just above the upper adaptor. However, it was discovered during these initial attempts that the load cell was not recording accurately the applied force. As explained earlier, the load cell device failed to cope with the high speed at which the load was applied and removed, especially at high frequencies (20 and $10 \mathrm{~Hz})$. Accordingly, the load cell was repositioned in contact with the lower hardened steel disc beneath the specimen. Force measurements were found to be more representative of that applied by the loading system even at high frequencies.

- Calibration. Considering the relatively small stress and strain magnitudes, proper calibration of all sensors connected to the system was important for obtaining accurately measured outputs (e.g., displacement or force). Figure 5.11 shows data collected with an extensometer not properly calibrated for a test performed at room temperature on an HMA 3 specimen at a loading frequency of $20 \mathrm{~Hz}$. The significant scatter of points constituting the strain signal made it impossible to accurately quantify the strain needed for characterizing the material response.

- Tuning. The tuning of the extensometer used in this experiment to control the loading mode is important for obtaining accurate measurements of displacements. Figure 5.12 shows results of a test performed using an extensometer not properly tuned, where it 
failed to record the test points that could be used to track the sinusoidal curve, particularly the signal peaks and valleys. However, using the proper tuning parameters (Gains), as described in Chapter 4, the sensors managed to accurately trace the strain signal as shown in Figure 5.13, including a smooth sinusoidal curve and a well-defined peak value.

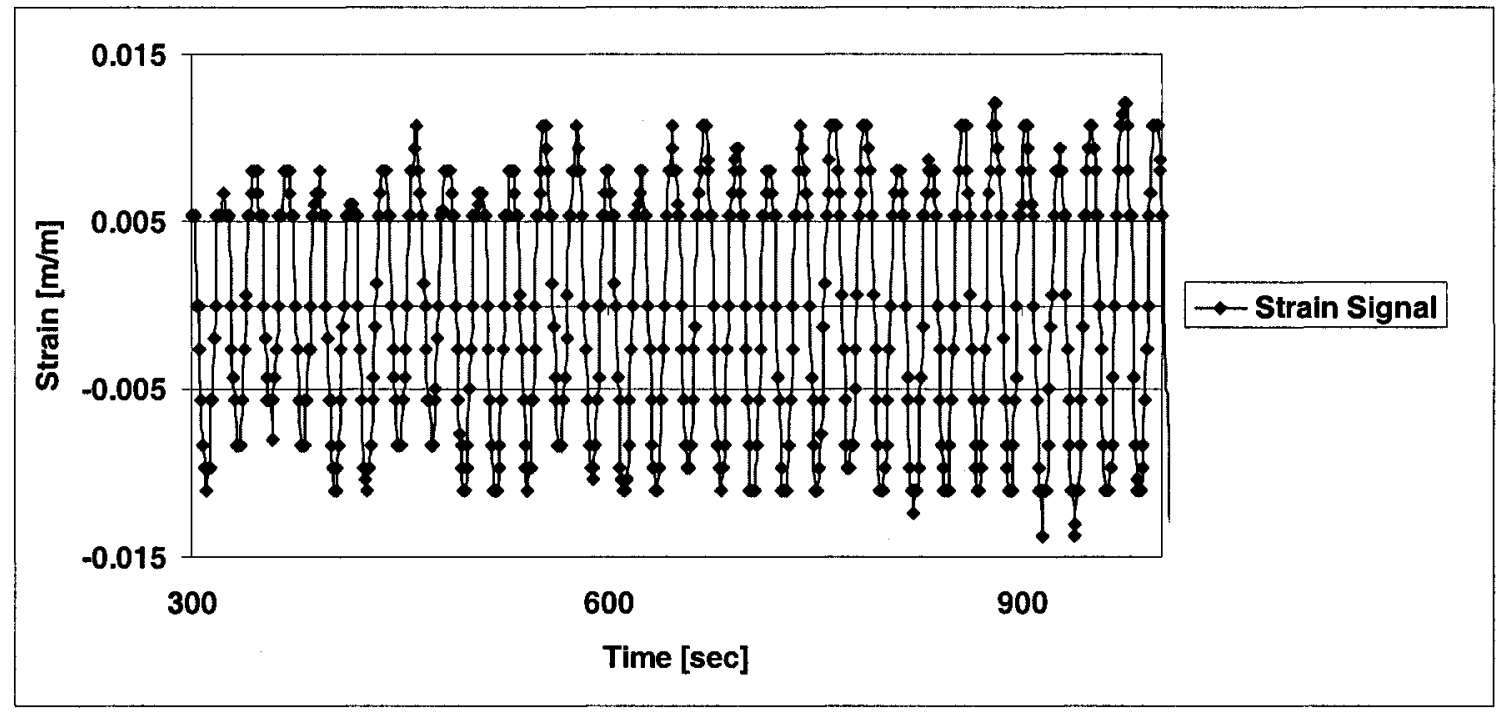

Figure 5.11. Strain signal for a test performed using an extensometer not well calibrated

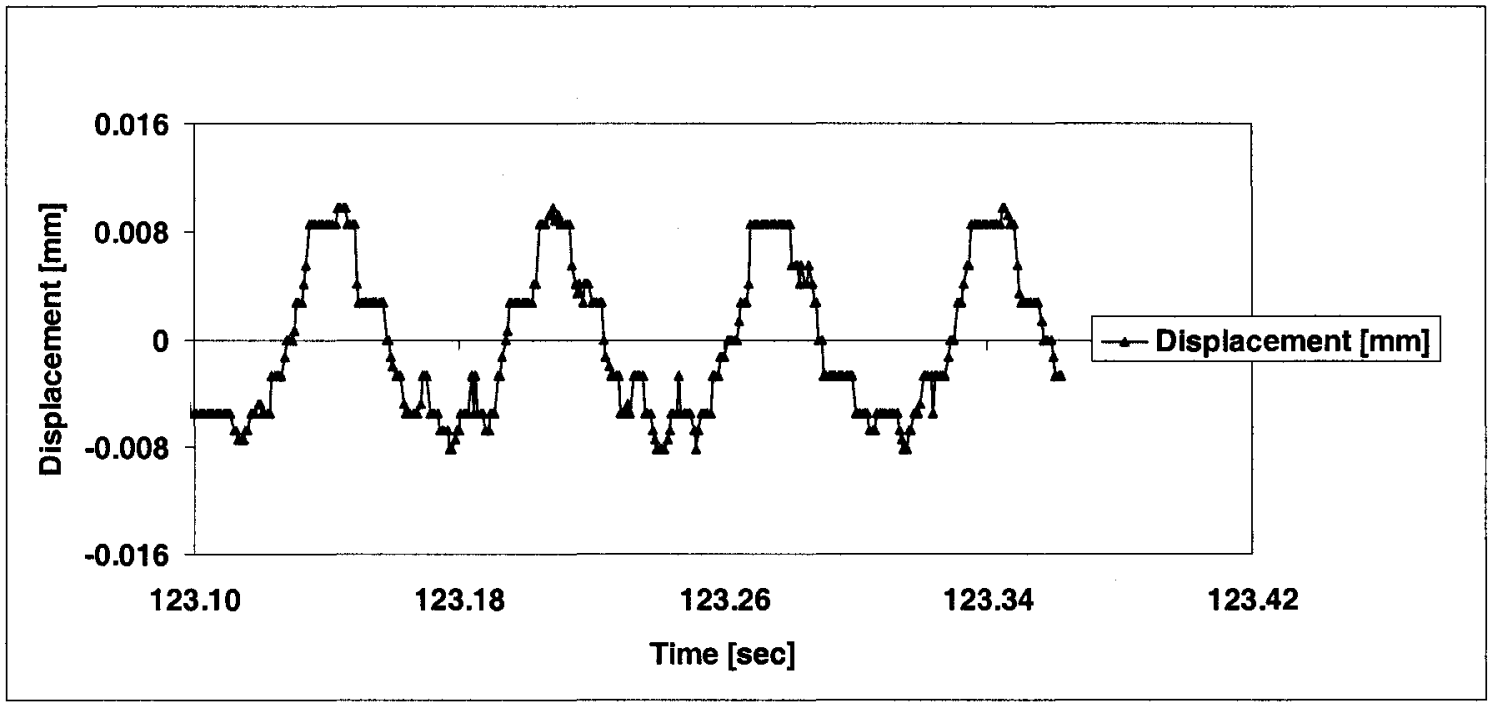

Figure 5.12. Strain signal for an extensometer not well tuned 


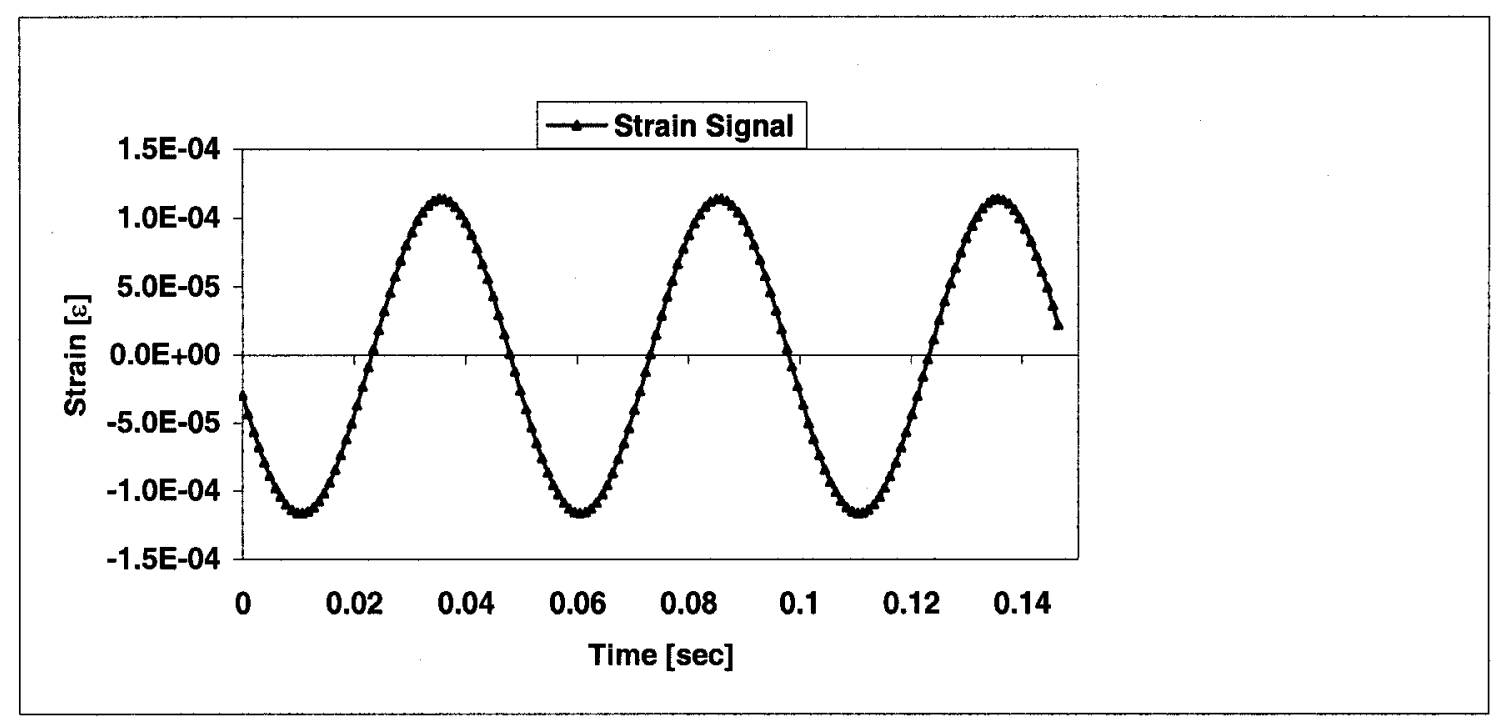

Figure 5.13. Strain signal for adjusted tuning parameters

The tests performed with the servo-hydraulic Material Testing System (MTS 810) involved a loading frame rated at $100 \mathrm{kN}$ with an integrally mounted 5-inch stroke actuator, and an electronic load cell with a capacity of $100 \mathrm{kN}$. The different components of the test set-up used in the laboratory investigation performed in this thesis are shown in Figure 5.14. The AC specimen prepared according to the process described in Chapter 4 was first glued from both ends to circular steel discs using an epoxy to keep it from moving during load applications. After 24 hours of curing, two extensometers were then attached to both sides of the glued specimen (at $180^{\circ}$ ) as shown in Figure 5.15. The steel discs were connected firmly to the two adaptors using two bolts. The test specimen and the attached accessories were then positioned inside the thermal chamber for temperature conditioning and to maintain the specimen at the desired test temperature. The hydraulic actuator was then brought down and attached to the upper adaptor. The test set-up ready for starting the test is shown in the schematic diagram in Figure 5.14. 
The MPT computer program, discussed earlier in Chapter 4, was then used to control the MTS system, perform the test according to the specifications set developed in this thesis and collect the raw data. Each $\mathrm{AC}$ test specimen was loaded at five different test temperatures; each set was performed in six different loading frequencies.

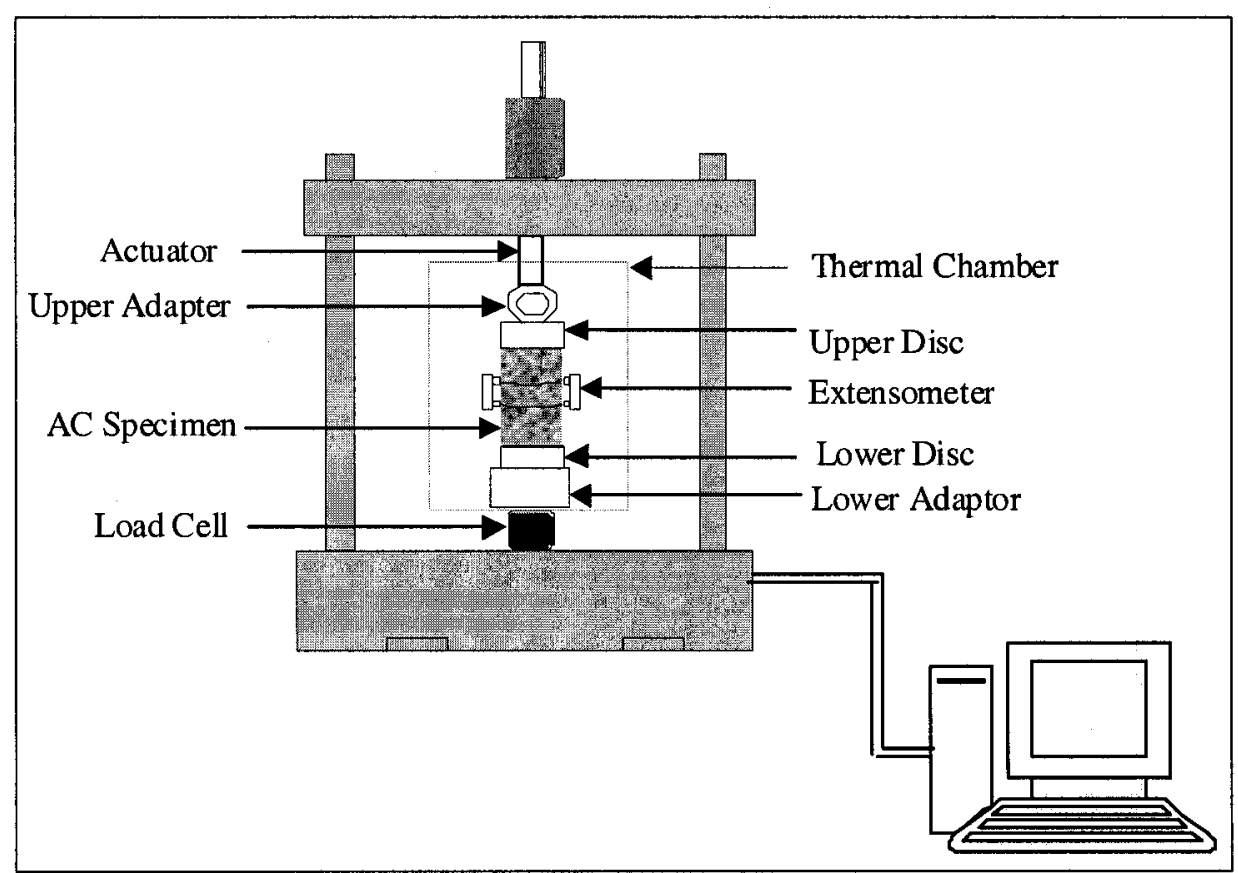

Figure 5.14. Complex modulus test set-up

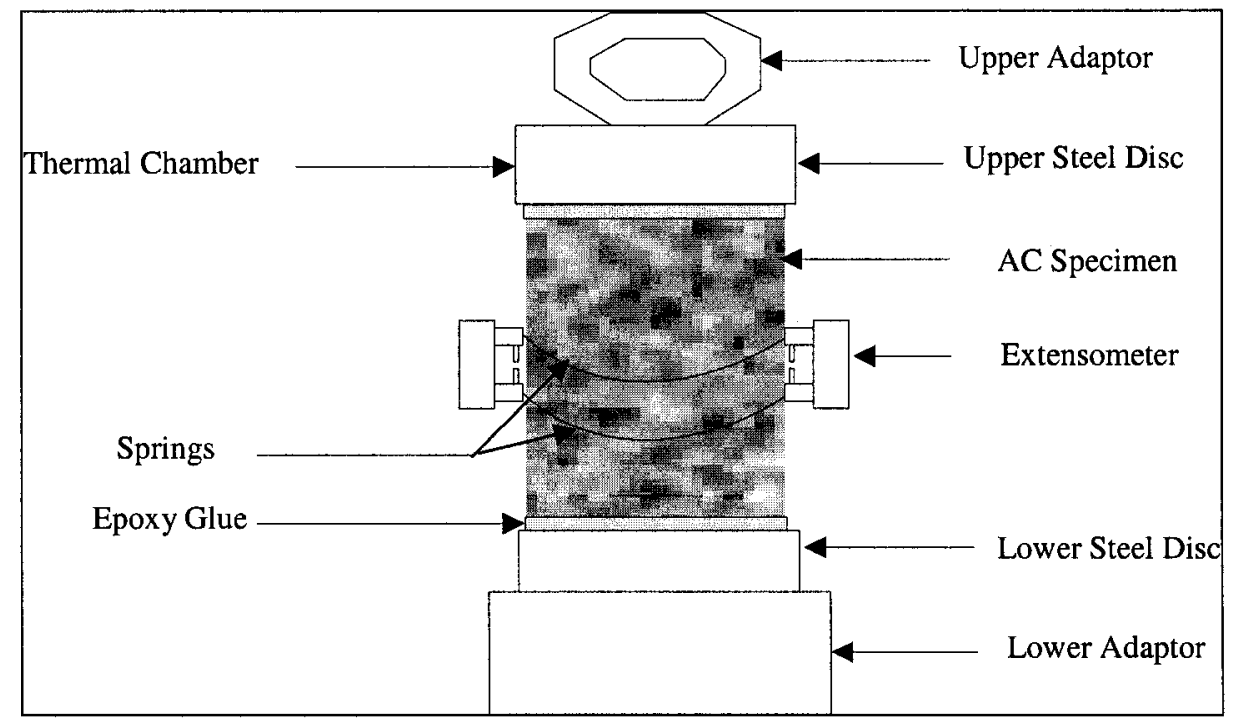

Figure 5.15. Asphalt concrete specimen ready for testing 


\subsection{Test Procedure}

After finalizing the test parameters discussed above and others mentioned earlier in Chapter 4, the MPT computer program is set to follow the adopted test parameters. With the sample positioned inside the thermal chamber, testing starts with conditioning the $\mathrm{AC}$ specimen at $-10^{\circ} \mathrm{C}$. At the end of the conditioning, which is pre-set for a 90 -minute period, the hydraulic actuator should be moved down to firmly connect with the upper adaptor glued to the sample. Refinements such as zeroing the load cell and the extensometers, and adjusting the tuning scales should be applied before starting the test. The test starts by pushing the START button, and the automated loading schedule incorporated into the MPT computer program will perform the test activities according to the intended sequence. The MPT software will adjust the strain gauge reading gradually to zero in 1 minute. This zero level will be maintained for another minute before applying the $20-\mathrm{Hz}$ loading frequency. The program will then stop loading and pause for a rest period of 2 minutes before proceeding to the next frequency, which is $10 \mathrm{~Hz}$. The test will proceed automatically (load - rest - load) until the end of the specified frequency range. Immediately after application of the $0.1-\mathrm{Hz}$ load frequency, the MPT program will stop the test.

The actuator should be disconnected manually from the sample and moved upward a reasonable distance, making sure it is not in contact with the sample. Then the new conditioning period should be initiated by setting the thermal controller at the next temperature. The same sequence discussed for testing at $-10^{\circ} \mathrm{C}$ should be applied using the appropriate MPT program developed for each temperature separately. The only difference between the testing steps at different test temperatures is in the rest period 
assigned between each frequency and the number of cycles needed to reach the intended strain amplitude. Longer rest periods and more cycles are needed at low temperatures and high frequencies than those required at high temperatures and low frequencies.

Table 5.2 summarizes the complex modulus test tasks and the sequence that should be followed. A detailed step-by-step test protocol was developed and is attached to this thesis as Appendix [A].

\subsection{Data Reduction Procedure}

The developed data reduction procedure consists of three major stages as described in Chapter 4 . Raw data were recorded by the data acquisition system incorporated into the MPT software mentioned earlier. The data acquisition system records forces detected by the load cell following a real time sequence (seconds). These are the forces associated with the applied displacements controlled by one extensometer. The other extensometer was abandoned because the MTS system failed to perform a dual control mode. A typical format for the data collected for a test performed at $20^{\circ} \mathrm{C}, 20 \mathrm{~Hz}$ using an HMA 2 mix prepared with a PG 64-34 binder, is shown in Table 5.3.this data is limited to $8.3 \mathrm{msec}$. A complete test at one temperature and frequency involves more than 5000 data points to draw the full stress-strain profile.

Data similar to that shown in Table 5.3, but corresponding to the entire test period, which usually includes more than 15000 rows, should then be processed in Stage 1 using an Excel-based macro (see the macro in Appendix C). Processing involves calculating stresses and strains from the last three cycles of each frequency using Equations 5.3 and 5.4. These last three cycles of each test frequency provide the most stable signals from the acquired data where the targeted strain amplitude is achieved. 
The typical raw data shown in Table 5.3 were processed using the macro and the results are shown in Table 5.4.

Table 5.2 Summary of the complex modulus test tasks

\begin{tabular}{|c|c|}
\hline Task \# & Steps \\
\hline $\begin{array}{l}\text { 1) Condition specimen at }-10^{\circ} \mathrm{C} \text { for } 90 \\
\text { minutes }\end{array}$ & $\begin{array}{l}\text { Manually, set the thermal chamber to cool down } \\
\text { the specimen to }-10^{\circ} \mathrm{C}\end{array}$ \\
\hline 2) Load specimen at $-10^{\circ} \mathrm{C}$ & 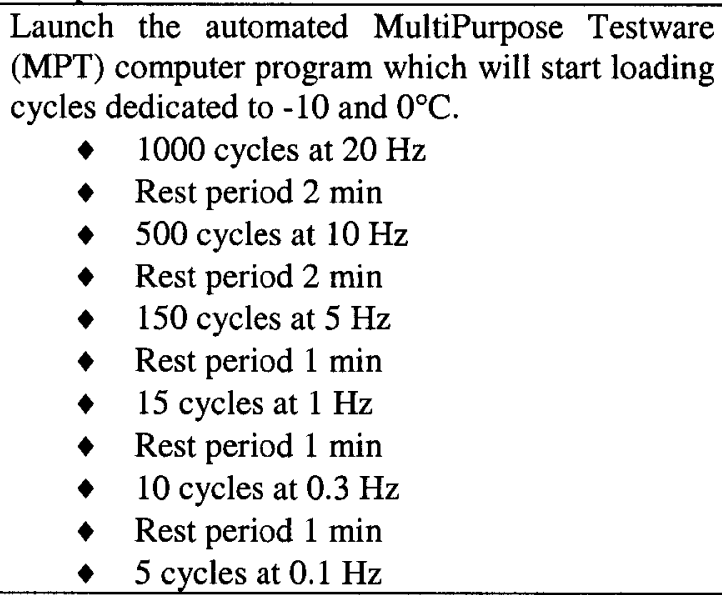 \\
\hline 3) Condition specimen at $0^{\circ} \mathrm{C}$ for 90 minutes & $\begin{array}{l}\text { Manually, set the thermal chamber to warm } \\
\text { specimen to } 0^{\circ} \mathrm{C}\end{array}$ \\
\hline 4) Load specimen at $0^{\circ} \mathrm{C}$ & Same as Task 2 \\
\hline $\begin{array}{l}\text { 5) Condition specimen at }+20^{\circ} \mathrm{C} \text { for } 90 \\
\text { minutes }\end{array}$ & $\begin{array}{l}\text { Manually, set the thermal chamber to warm } \\
\text { specimen to }+20^{\circ}\end{array}$ \\
\hline 6) Testing at $+20^{\circ} \mathrm{C}$ & 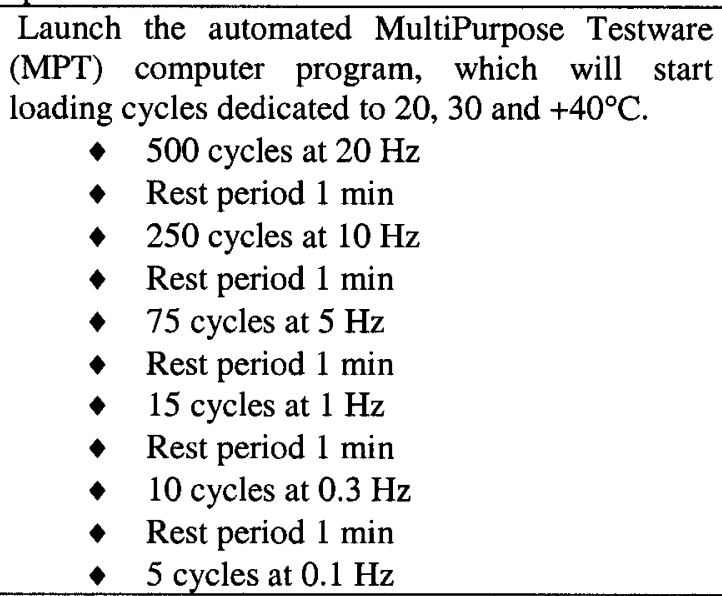 \\
\hline $\begin{array}{l}\text { 7) Conditioning the specimen at } 30^{\circ} \mathrm{C} \text { for } 90 \\
\text { minutes }\end{array}$ & $\begin{array}{l}\text { Manually, set the thermal chamber to warm } \\
\text { specimen to }+30^{\circ} \mathrm{C} \text {. }\end{array}$ \\
\hline 8) Load specimen at $+30^{\circ} \mathrm{C}$ & Same as Task 6 \\
\hline $\begin{array}{l}\text { 9) Condition specimen at }+40^{\circ} \mathrm{C} \text { for } 90 \\
\text { minutes }\end{array}$ & $\begin{array}{l}\text { Manually, set the thermal chamber to warm } \\
\text { specimen to }+40^{\circ} \mathrm{C} \text {. }\end{array}$ \\
\hline 10) Load specimen at $+40^{\circ} \mathrm{C}$ & Same as Task 6 \\
\hline
\end{tabular}


$\sigma_{0}=\frac{\mathrm{P}}{\mathrm{A}}$

where $A$ is the cross-sectional area $\left(\mathrm{mm}^{2}\right)$ of a specimen with $100-\mathrm{mm}$ diameter, and

$P$ is the recorded axial force, $(\mathrm{N})$

$\varepsilon_{0}=\frac{\Delta}{\mathrm{L}}$

where $\Delta$ is the measured displacement in $\mathrm{mm}$, and

$L$ is the gauge length of the extensometer used to control the test which is equivalent to $25 \mathrm{~mm}$.

Table 5.3. Typical acquired raw data

\begin{tabular}{|c|c|c|}
\hline $\begin{array}{c}\text { Time } \\
\mathbf{( S e c})\end{array}$ & $\begin{array}{c}\text { Axial Ram } \\
\text { Force }(\mathbf{N})\end{array}$ & $\begin{array}{c}\text { Axial Displacements } \\
\text { Ext } \mathbf{1}(\mathbf{m m})\end{array}$ \\
\hline 169.8801 & -20625 & $-1.98 \mathrm{E}-03$ \\
\hline 169.8806 & -21105 & $-2.13 \mathrm{E}-03$ \\
\hline 169.8811 & -21545 & $-2.19 \mathrm{E}-03$ \\
\hline 169.8816 & -21892 & $-2.23 \mathrm{E}-03$ \\
\hline 169.8821 & -22175 & $-2.33 \mathrm{E}-03$ \\
\hline 169.8826 & -22430 & $-2.40 \mathrm{E}-03$ \\
\hline 169.8831 & -22613 & $-2.36 \mathrm{E}-03$ \\
\hline 169.8835 & -22706 & $-2.30 \mathrm{E}-03$ \\
\hline 169.884 & -22736 & $-2.45 \mathrm{E}-03$ \\
\hline 169.8845 & -22714 & $-2.45 \mathrm{E}-03$ \\
\hline 169.885 & -22616 & $-2.47 \mathrm{E}-03$ \\
\hline 169.8855 & -22426 & $-2.40 \mathrm{E}-03$ \\
\hline 169.886 & -22201 & $-2.39 \mathrm{E}-03$ \\
\hline 169.8865 & -21955 & $-2.48 \mathrm{E}-03$ \\
\hline 169.887 & -21623 & $-2.35 \mathrm{E}-03$ \\
\hline 169.8875 & -21221 & $-2.30 \mathrm{E}-03$ \\
\hline 169.8879 & -20761 & $-2.22 \mathrm{E}-03$ \\
\hline 169.8884 & -20229 & $-2.34 \mathrm{E}-03$ \\
\hline
\end{tabular}

The data processed in Stage 1 are then subjected to further processing in Stage 2 using the statistical package "TableCurve" to obtain amplitudes of stresses and strains in 
addition to the phase angle of each stress and strain signal for the entire sweep of test temperatures and frequencies.

Table 5.4. Data from Table 5.3 processed using a macro

\begin{tabular}{|c|c|c|}
\hline $\begin{array}{c}\text { Time } \\
(\mathbf{s e c})\end{array}$ & $\begin{array}{c}\text { Stress }\left(\sigma_{\mathrm{o}}\right) \\
{[\mathbf{M P a})}\end{array}$ & $\begin{array}{c}\text { Strain }\left(\varepsilon_{\mathrm{o}}\right) \_ \text {Ext 1 } \\
{[\varepsilon]}\end{array}$ \\
\hline $5.4 \mathrm{E}-03$ & -2.63 & $-7.9 \mathrm{E}-05$ \\
\hline $5.9 \mathrm{E}-03$ & -2.69 & $-8.5 \mathrm{E}-05$ \\
\hline $6.3 \mathrm{E}-03$ & -2.74 & $-8.8 \mathrm{E}-05$ \\
\hline $6.8 \mathrm{E}-03$ & -2.79 & $-8.9 \mathrm{E}-05$ \\
\hline $7.3 \mathrm{E}-03$ & -2.82 & $-9.3 \mathrm{E}-05$ \\
\hline $7.8 \mathrm{E}-03$ & -2.85 & $-9.6 \mathrm{E}-05$ \\
\hline $8.3 \mathrm{E}-03$ & -2.88 & $-9.4 \mathrm{E}-05$ \\
\hline $8.8 \mathrm{E}-03$ & -2.89 & $-9.2 \mathrm{E}-05$ \\
\hline $9.3 \mathrm{E}-03$ & -2.89 & $-9.8 \mathrm{E}-05$ \\
\hline $9.8 \mathrm{E}-03$ & -2.89 & $-9.8 \mathrm{E}-05$ \\
\hline $1.0 \mathrm{E}-02$ & -2.88 & $-9.9 \mathrm{E}-05$ \\
\hline $1.1 \mathrm{E}-02$ & -2.85 & $-9.6 \mathrm{E}-05$ \\
\hline $1.1 \mathrm{E}-02$ & -2.83 & $-9.6 \mathrm{E}-05$ \\
\hline $1.2 \mathrm{E}-02$ & -2.79 & $-9.9 \mathrm{E}-05$ \\
\hline $1.2 \mathrm{E}-02$ & -2.75 & $-9.4 \mathrm{E}-05$ \\
\hline $1.3 \mathrm{E}-02$ & -2.70 & $-9.2 \mathrm{E}-05$ \\
\hline $1.3 \mathrm{E}-02$ & -2.64 & $-8.9 \mathrm{E}-05$ \\
\hline $1.4 \mathrm{E}-02$ & -2.57 & $-9.3 \mathrm{E}-05$ \\
\hline
\end{tabular}

The "TableCurve" quantifies the amplitudes of stresses and strains in addition to the phase angle of each stress and strain signal mathematically in terms of the coefficients shown in Equations 4.5 and 4.6, mentioned in Chapter 4 and reproduced below:

$$
\begin{aligned}
& \sigma=a_{1}+b_{1} \sin \left(2 \pi t / d_{1}+c_{1}\right) \\
& \varepsilon=a_{2}+b_{2} \sin \left(2 \pi t / d_{2}+c_{2}\right)
\end{aligned}
$$

where: $\sigma$ and $\varepsilon$ are the stress and strain respectively at time t,

$b_{1}$ and $b_{2}$ represent the amplitude of stress and strain $\sigma_{o}$ and $\varepsilon_{o}$ respectively, $a_{1}$ and $a_{2}$ are regression constants, 
$c_{1}$ and $c_{2}$ represent individual phase angles of stress and strain wave functions $\phi_{1}$ and $\phi_{2}$ respectively.

Results of the typical data shown earlier in Table 5.4, processed using "TableCurve" to calculate stress and strain amplitudes, are shown in Table 5.5.

Processing of data in Stage 3 involves calculating the dynamic modulus and phase angles using the output of Stage 2. The dynamic modulus $\left|E^{*}\right|$ is calculated using Equation 3.11, mentioned in Chapter 3 and reproduced below:

$$
\left|E^{*}\right|=\frac{\sigma_{0}}{\varepsilon_{0}}
$$

The phase lag $\phi$ between signals associated with the applied stress and the corresponding strain should be calculated as the difference between $\phi_{l}$ and $\phi_{2}$ in radians. The phase lag in radians is then converted into phase angle in degrees according to Equation 5.5:

$\phi=\left(\phi_{1}-\phi_{2}\right) * 180 / \pi$

A typical output of data processed in Stage 3 using the "TableCurve" is shown in Table 5.6.

Table 5.5. Data processed using the "TableCurve"

\begin{tabular}{|c|c|c|c|c|}
\hline $\begin{array}{c}\text { Frequency } \\
(\mathrm{Hz})\end{array}$ & $\begin{array}{c}\text { Stress } \\
\sigma_{\mathrm{a}} \mathrm{MPa}\end{array}$ & $\begin{array}{c}\text { Phase Angle of } \\
\text { Stress Cycle }\left(\phi_{1}\right)\end{array}$ & $\begin{array}{c}\text { Strain } \\
\varepsilon_{\mathrm{o}}(\mu \varepsilon)\end{array}$ & $\begin{array}{c}\text { Phase Angle of } \\
\text { Strain Cycle }\left(\phi_{2}\right)\end{array}$ \\
\hline 20 & 0.50 & 4.1 & 101 & 3.7 \\
\hline 10 & 0.39 & 5.0 & 100 & 4.6 \\
\hline 5 & 0.33 & 5.3 & 101 & 4.9 \\
\hline 1 & 0.22 & 5.2 & 100 & 4.6 \\
\hline 0.3 & 0.16 & 5.3 & 100 & 4.7 \\
\hline 0.1 & 0.12 & 5.3 & 100 & 4.7 \\
\hline
\end{tabular}


Table 5.6. Dynamic modulus and phase angle calculated using output of data processed using the "TableCurve"

\begin{tabular}{|c|c|c|}
\hline Frequency $(\mathrm{Hz})$ & $\left|E^{*}\right|(\mathrm{MPa})$ & Phase Angle $\phi[\mathrm{deg}]$ \\
\hline 20 & $4.09 \mathrm{E}+03$ & 25.0 \\
\hline 10 & $3.28 \mathrm{E}+03$ & 27.2 \\
\hline 5 & $2.76 \mathrm{E}+03$ & 28.1 \\
\hline 1 & $1.87 \mathrm{E}+03$ & 31.0 \\
\hline 0.3 & $1.37 \mathrm{E}+03$ & 33.0 \\
\hline 0.1 & $1.03 \mathrm{E}+03$ & 34.0 \\
\hline
\end{tabular}

\subsection{Characterization Using Processed Data}

Characterization of $\mathrm{AC}$ materials using data processed according to the above stages is discussed in detail in Chapter 6. Characterization is discussed in this section only because it is being considered as part of the test protocol and data processing procedure added to this thesis as Appendix A. The characterization technique involves calculating the storage and loss moduli using Equations 3.12 and 3.13.

$\mathrm{E}_{1}=\left|E^{*}\right| \cos \phi$

$\mathrm{E}_{2}=\left|E^{*}\right| \sin \phi$

$E_{1}$ and $E_{2}$ are the storage and loss moduli respectively, and the other parameters were defined previously in Chapter 3.

The dynamic moduli and phase angles, determined at five temperatures and six frequencies can be used to determine eight parameters included in the Huet-Sayegh rheological model that characterize the $\mathrm{AC}$ response as described in Chapter 3 . In this thesis the processed data was plugged directly into the HUSAROAD software mentioned in Chapter 4 to yield the eight parameters of the Huet-Sayegh model including $E_{0}, E_{\infty} \delta$, $k, h, a, b$, and $c$. Table 5.7 shows the eight parameters obtained using the HUSAROAD 
software for tests performed on two similar specimens prepared from an HMA 2 mix using a PG 64-34 binder. However, detailed characterization of the mixes used in this thesis are provided in Chapter 6 and in the established Material Library added to this thesis as Appendix B.

Table 5.7. Typical results of Huet-Sayegh model parameters calculated by the HUSAROAD software

\begin{tabular}{|l|l|l|l|l|l|l|l|l|}
\hline Sample ID & $E_{\infty}$ & $E_{o}$ & $\delta$ & $k$ & $h$ & $a$ & $b$ & $c$ \\
\hline HMA 2 64-34-1 & 17480 & 20 & 3.40 & 0.27 & 0.57 & -1.02 & -0.26 & 0.001 \\
\hline HMA 2 64-34-2 & 17971 & 29 & 2.21 & 0.30 & 0.54 & -1.53 & -0.29 & 0.001 \\
\hline Average & 17726 & 24.5 & 2.80 & 0.29 & 0.56 & -1.27 & -0.28 & 0.001 \\
\hline
\end{tabular}

\subsection{Summary}

The conceptualized complex modulus test procedure was evaluated in this chapter and a test protocol was developed to assist in producing the mechanistic properties of common AC mixes. The output of tests performed on different mixes using the test protocol is presented and analyzed in Chapter 6. Added to the test protocol are data processing procedures (Stages 1 to 3 ) to reduce raw data and prepare the results needed to complete the characterization process based on the Huet-Sayegh rheological model. Direct application of the data in other models, such as ASSHTO 2002 Design Guide, will also be possible since the basic complex modulus results are made available in the developed Material Library, which will be discussed in Chapter 7. 


\section{CHAPTER 6 \\ RESULTS AND ANALYSIS}

\subsection{Introduction}

The response of bituminous mixtures is significantly affected by exposure conditions including those encountered during construction and in-service conditions (traffic and the environment). An ideal characterization technique is expected to capture known responses of an $\mathrm{AC}$ material subjected to different exposure conditions in the field including traffic-related variables (vehicle weight characteristics and traffic speed), temperature variations (cold versus warm and rate of cooling). Also critical is the sensitivity of the characterization technique to $\mathrm{AC}$ material properties as influenced by the properties of the mix components (aggregate and binder). The characterization technique should reflect the viscous nature of binders, which results in a time-dependent response where the strain lags the stress. AC response in the field has been discussed in detail in Chapter 3 and the results of the laboratory experiment related to the $\mathrm{AC}$ response to various exposure conditions are discussed below.

The proposed mechanistic characterization technique is based on the complex modulus approach and utilizes two parameters, namely the dynamic modulus and the phase angle, to capture the $\mathrm{AC}$ response. The dynamic modulus is used as an indication of the $\mathrm{AC}$ stiffness while the phase angle reflects the viscous component of the response. This chapter discusses results of tests conducted to quantify these two parameters using results of the complex modulus test procedure developed in Chapter 5. The results were used to convey the effectiveness of the proposed characterization technique as reflected in its responsiveness to variables associated with exposure conditions simulated during the 
laboratory investigation and construction quality controlled during the manufacturing of test specimens. A sensitivity analysis was performed to examine the effectiveness of the proposed characterization technique following the experimental plan discussed in Chapter 4 and benefiting from data generated within the range of variables summarized below in Table 6.1 .

Table 6.1. Factors that influence $\mathrm{AC}$ characteristic response

\begin{tabular}{|c|l|l|}
\hline NO. & Elements of the Investigation & Range of the Investigated variables \\
\hline 1 & $\begin{array}{l}\text { Traffic Characteristics } \\
\text { (Frequency to reflect vehicle speed) }\end{array}$ & $20,10,5,1,0.3$, and $0.1 \mathrm{~Hz}$ \\
\hline 2 & $\begin{array}{l}\text { Environmental Condition } \\
\text { (Temperature variations) }\end{array}$ & $-10,0,20,30$, and $40^{\circ} \mathrm{C}$ \\
\hline 3 & Mix type & HMA 1, HMA 2, HMA 3, SP 1 and SP 2 \\
\hline 4 & Binder type & PG 52-34, PG 58-22 and PG 64-34 \\
\hline 5 & $\begin{array}{l}\text { Construction Quality } \\
\text { (Percent air voids) }\end{array}$ & $3.5,6.0,7.2$, and 10\% \\
\hline 6 & Curing (Age) & $1,6,16$, and 30 days \\
\hline 7 & Mix Design Approach & Marshall and SuperPave \\
\hline
\end{tabular}

\subsection{Determination of Complex Modulus Parameters}

The raw data acquired from tests conducted using the test protocol developed in Chapter 5 was processed following several stages prior to obtaining the complex modulus parameters that can be used to characterize the response of $\mathrm{AC}$ materials. The following paragraphs show numerical examples showing the data reduction process performed on an HMA 2 mix prepared using a PG 52-34 binder.

\subsubsection{Collecting Raw Data}

The first step is to save the raw data collected by the data acquisition system discussed earlier in Chapter 5. Table 6.2 shows real data collected during the test at a loading frequency of $20 \mathrm{~Hz}$ for a test performed at a test temperature of $0^{\circ} \mathrm{C}$. 
Table 6.2. Typical raw data collected at $20 \mathrm{~Hz}, 0^{\circ} \mathrm{C}$

\begin{tabular}{|c|c|c|}
\hline \multicolumn{3}{|c|}{ Data Acquisition of Loading Frequency of $20 \mathrm{~Hz}$} \\
\hline $\begin{array}{l}\text { Time } \\
{[\mathrm{sec}]}\end{array}$ & $\begin{array}{c}\text { Axial Ram Force } \\
{[\mathrm{N}]}\end{array}$ & $\begin{array}{c}\text { Axial Displacement } \\
{[\mathrm{mm}]}\end{array}$ \\
\hline 167.582 & -8467 & $-2.3 \mathrm{E}-03$ \\
\hline 167.582 & -8506 & $-2.2 \mathrm{E}-03$ \\
\hline 167.583 & -8522 & $-2.5 \mathrm{E}-03$ \\
\hline 167.583 & -8491 & $-2.4 \mathrm{E}-03$ \\
\hline 167.584 & -8400 & $-2.4 \mathrm{E}-03$ \\
\hline 167.584 & -8309 & $-2.5 \mathrm{E}-03$ \\
\hline 167.585 & -8202 & $-2.5 \mathrm{E}-03$ \\
\hline 167.585 & -8035 & $-2.4 \mathrm{E}-03$ \\
\hline 167.586 & -7823 & $-2.4 \mathrm{E}-03$ \\
\hline 167.586 & -7600 & $-2.5 \mathrm{E}-03$ \\
\hline 167.586 & -7336 & $-2.4 \mathrm{E}-03$ \\
\hline 167.587 & -7046 & $-2.3 \mathrm{E}-03$ \\
\hline 167.587 & -6717 & $-2.3 \mathrm{E}-03$ \\
\hline 167.588 & -6379 & $-2.2 \mathrm{E}-03$ \\
\hline 167.588 & -6028 & $-2.2 \mathrm{E}-03$ \\
\hline 167.589 & -5630 & $-2.1 \mathrm{E}-03$ \\
\hline 167.589 & -5203 & $-2.0 \mathrm{E}-03$ \\
\hline 167.59 & -4783 & $-2.0 \mathrm{E}-03$ \\
\hline
\end{tabular}

\subsubsection{Data Processing Using an Excel-Based Macro}

In this study, acquired data similar to those shown in Table 6.2 are then processed using an Excel-based macro to obtain stresses and strains for the last three cycles of each loading frequency. A typical result for data processed using the macro is shown in Table 6.3 in terms of stresses and strains.

\subsubsection{Refinement of Stress and Strain Signals using "TableCurve"}

The preliminary data processed as shown in Table 6.3 are then subjected to further processing using the statistical package "TableCurve" (as mentioned in Chapter 5) to obtain the amplitudes of the stresses and strains in addition to the lag phase of each stress 
and strain signal. Table 6.4 shows stress data after processing the signal using the "TableCurve."

Table 6.3. Processed data using an Excel macro

\begin{tabular}{|c|c|c|}
\hline Processed Time [Sec] & Stress [MPa] & Strain Ext $1[\mathrm{~mm} / \mathrm{mm}]$ \\
\hline$-4.8 \mathrm{E}-06$ & -0.77 & $-4.78 \mathrm{E}-05$ \\
\hline $4.9 \mathrm{E}-04$ & -0.81 & $-5.26 \mathrm{E}-05$ \\
\hline $9.8 \mathrm{E}-04$ & -0.86 & $-6.15 \mathrm{E}-05$ \\
\hline $1.5 \mathrm{E}-03$ & -0.91 & $-6.51 \mathrm{E}-05$ \\
\hline $2.0 \mathrm{E}-03$ & -0.94 & $-6.68 \mathrm{E}-05$ \\
\hline $2.4 \mathrm{E}-03$ & -0.98 & $-7.47 \mathrm{E}-05$ \\
\hline $2.9 \mathrm{E}-03$ & -1.01 & $-7.63 \mathrm{E}-05$ \\
\hline $3.4 \mathrm{E}-03$ & -1.03 & $-7.84 \mathrm{E}-05$ \\
\hline $3.9 \mathrm{E}-03$ & -1.05 & $-8.48 \mathrm{E}-05$ \\
\hline $4.4 \mathrm{E}-03$ & -1.07 & $-8.72 \mathrm{E}-05$ \\
\hline 4.9E- 03 & -1.08 & $-8.85 \mathrm{E}-05$ \\
\hline $5.4 \mathrm{E}-03$ & -1.09 & $-9.30 \mathrm{E}-05$ \\
\hline $5.9 \mathrm{E}-03$ & -1.09 & $-9.34 \mathrm{E}-05$ \\
\hline $6.3 \mathrm{E}-03$ & -1.09 & $-9.47 \mathrm{E}-05$ \\
\hline $6.8 \mathrm{E}-03$ & -1.08 & $-9.72 \mathrm{E}-05$ \\
\hline 7.3E-03 & -1.07 & $-1.01 \mathrm{E}-04$ \\
\hline $7.8 \mathrm{E}-03$ & -1.06 & $-9.61 \mathrm{E}-05$ \\
\hline 8.3E-03 & -1.04 & $-9.88 \mathrm{E}-05$ \\
\hline
\end{tabular}

Table 6.4. Stress amplitudes adjusted using "TableCurve"

\begin{tabular}{|c|c|c|c|c|}
\hline $\begin{array}{c}\text { Observed Time } \\
{[\text { Sec] }}\end{array}$ & $\begin{array}{c}\text { Observed Stress } \\
{[\mathrm{MPa}]}\end{array}$ & $\begin{array}{c}\text { Predicted Stress } \\
{[\mathrm{MPa}]}\end{array}$ & $\begin{array}{c}\text { Residual Stress } \\
{[\mathrm{MPa}]}\end{array}$ & $\begin{array}{c}\text { Residual Stress } \\
\%\end{array}$ \\
\hline$-4.8 \mathrm{E}-06$ & -0.77 & -0.76 & -0.01 & 1.41 \\
\hline $4.9 \mathrm{E}-04$ & -0.82 & -0.81 & -0.01 & 1.44 \\
\hline $9.8 \mathrm{E}-04$ & -0.86 & -0.85 & -0.02 & 1.83 \\
\hline $1.5 \mathrm{E}-03$ & -0.91 & -0.89 & -0.02 & 2.42 \\
\hline $2.0 \mathrm{E}-03$ & -0.94 & -0.92 & -0.02 & 2.64 \\
\hline $2.4 \mathrm{E}-03$ & -0.98 & -0.95 & -0.03 & 2.71 \\
\hline $2.9 \mathrm{E}-03$ & -1.01 & -0.98 & -0.03 & 2.96 \\
\hline $3.4 \mathrm{E}-03$ & -1.03 & -1.00 & -0.03 & 3.22 \\
\hline $3.9 \mathrm{E}-03$ & -1.05 & -1.02 & -0.03 & 3.29 \\
\hline $4.4 \mathrm{E}-03$ & -1.07 & -1.04 & -0.03 & 3.17 \\
\hline $4.9 \mathrm{E}-03$ & -1.08 & -1.05 & -0.03 & 3.19 \\
\hline $5.4 \mathrm{E}-03$ & -1.09 & -1.05 & -0.04 & 3.27 \\
\hline $5.9 \mathrm{E}-03$ & -1.09 & -1.06 & -0.03 & 3.15 \\
\hline $6.3 \mathrm{E}-03$ & -1.09 & -1.06 & -0.03 & 2.82 \\
\hline $6.8 \mathrm{E}-03$ & -1.08 & -1.05 & -0.03 & 2.71 \\
\hline $7.3 \mathrm{E}-03$ & -1.07 & -1.04 & -0.03 & 2.80 \\
\hline $7.8 \mathrm{E}-03$ & -1.06 & -1.03 & -0.03 & 2.53 \\
\hline $8.3 \mathrm{E}-03$ & -1.04 & -1.01 & -0.02 & 2.27 \\
\hline
\end{tabular}


The output of the computational process performed by the "TableCurve" statistical package applied to the data collected from the test for stress cycles is the information shown in Table 6.5.

Table 6.5. Adjusted stress amplitude as produced by "TableCurve"

\begin{tabular}{|c|c|c|c|}
\hline & [Sine] $y=a+b \sin$ & Parameters & Values \\
\hline Equation & $(2 \mathrm{px} / \mathrm{d}+\mathrm{c})$ & $\mathrm{a}_{1}$ & 0.033 \\
\hline Eqn \# & 8014 & $\mathrm{~b}_{1}$ & $\begin{array}{c}1.091 \text { (stress amplitude, } \\
\mathrm{MPa} \text { ) }\end{array}$ \\
\hline $\mathrm{R}^{2}$ & 0.999 & $\mathrm{c}_{1}$ & 3.959 (phase lag, radians) \\
\hline DF Adj $R^{2}$ & 0.999 & $d_{1}$ & 0.05 \\
\hline Fit Std Err & 0.0217 & & \\
\hline F-stat & 127850 & & \\
\hline Date & Dec 8, 2004 & & \\
\hline Time & 12:17:07 PM & & \\
\hline
\end{tabular}

The right-hand side of Table 6.5 shows results of statistical analysis related to the accuracy of collected data obtained using "TableCurve". The left-hand side consists of quantitative information related to the stress amplitude referred to as " $\mathrm{b}_{1}$ " and the phase lag in the stress signal referred to as " $\mathrm{c}_{1}$ ".

The same procedure followed to process the stress data was applied to the strain data. Table 6.6 shows results of the processed strain signal produced using "TableCurve."

Table 6.6. Strain amplitudes adjusted using "TableCurve"

\begin{tabular}{|c|c|c|c|c|}
\hline $\begin{array}{c}\text { Time Observed } \\
(\mathrm{Sec})\end{array}$ & $\begin{array}{c}\text { Strain Observed } \\
{[\mathrm{mm} / \mathrm{mm}]}\end{array}$ & $\begin{array}{c}\text { Strain Predicted } \\
{[\mathrm{mm} / \mathrm{mm}]}\end{array}$ & $\begin{array}{c}\text { Strain Residual } \\
{[\mathrm{mm} / \mathrm{mm}]}\end{array}$ & $\begin{array}{c}\text { Strain Residual } \\
\%\end{array}$ \\
\hline$-4.8 \mathrm{E}-06$ & $-4.8 \mathrm{E}-05$ & $-4.7 \mathrm{E}-05$ & $-6.4 \mathrm{E}-07$ & 1.3 \\
\hline $4.9 \mathrm{E}-04$ & $-5.3 \mathrm{E}-05$ & $-5.2 \mathrm{E}-05$ & $-2.2 \mathrm{E}-07$ & 0.4 \\
\hline $9.8 \mathrm{E}-04$ & $-6.2 \mathrm{E}-05$ & $-5.7 \mathrm{E}-05$ & $-4.2 \mathrm{E}-06$ & 6.8 \\
\hline $1.5 \mathrm{E}-03$ & $-6.5 \mathrm{E}-05$ & $-6.2 \mathrm{E}-05$ & $-3.0 \mathrm{E}-06$ & 4.6 \\
\hline $2.0 \mathrm{E}-03$ & $-6.7 \mathrm{E}-05$ & $-6.7 \mathrm{E}-05$ & $-2.2 \mathrm{E}-07$ & 0.3 \\
\hline $2.4 \mathrm{E}-03$ & $-7.5 \mathrm{E}-05$ & $-7.1 \mathrm{E}-05$ & $-3.9 \mathrm{E}-06$ & 5.2 \\
\hline $2.9 \mathrm{E}-03$ & $-7.6 \mathrm{E}-05$ & $-7.5 \mathrm{E}-05$ & $-1.6 \mathrm{E}-06$ & 2.1 \\
\hline $3.4 \mathrm{E}-03$ & $-7.8 \mathrm{E}-05$ & $-7.8 \mathrm{E}-05$ & $-2.9 \mathrm{E}-08$ & 0.0 \\
\hline $3.9 \mathrm{E}-03$ & $-8.5 \mathrm{E}-05$ & $-8.2 \mathrm{E}-05$ & $-3.0 \mathrm{E}-06$ & 3.6 \\
\hline $4.4 \mathrm{E}-03$ & $-8.7 \mathrm{E}-05$ & $-8.5 \mathrm{E}-05$ & $-2.4 \mathrm{E}-06$ & 2.8 \\
\hline $4.9 \mathrm{E}-03$ & $-8.9 \mathrm{E}-05$ & $-8.8 \mathrm{E}-05$ & $-9.6 \mathrm{E}-07$ & 1.1 \\
\hline $5.4 \mathrm{E}-03$ & $-9.3 \mathrm{E}-05$ & $-9.0 \mathrm{E}-05$ & $-3.0 \mathrm{E}-06$ & 3.2 \\
\hline $5.9 \mathrm{E}-03$ & $-9.3 \mathrm{E}-05$ & $-9.2 \mathrm{E}-05$ & $-1.4 \mathrm{E}-06$ & 1.5 \\
\hline
\end{tabular}


The output of the strain computational process performed using the statistical package

"TableCurve" is shown in Table 6.7.

Table 6.7. Adjusted stress amplitude as produced by "TableCurve"

\begin{tabular}{|c|c|}
\hline Equation & $\begin{array}{c}\text { [Sine] } \mathrm{y}=\mathrm{a}+\mathrm{b} \sin \\
(2 \mathrm{px} / \mathrm{d}+\mathrm{c})\end{array}$ \\
\hline Eqn \# & 8014 \\
\hline $\mathrm{R}^{2}$ & 0.999 \\
\hline $\begin{array}{c}\text { DF Adj } \\
\mathrm{R}^{2}\end{array}$ & 0.999 \\
\hline $\begin{array}{c}\text { Fit Std } \\
\text { Err }\end{array}$ & $2.17 \mathrm{E}-06$ \\
\hline F-stat & 105573 \\
\hline Date & Dec 8,2004 \\
\hline Time & $12: 53: 47 \mathrm{PM}$ \\
\hline
\end{tabular}

\begin{tabular}{|c|c|}
\hline Parameters & Values \\
\hline $\mathrm{a}_{2}$ & $1.91 \mathrm{E}-06$ \\
\hline $\mathrm{b}_{2}$ & $\begin{array}{c}9.87 \mathrm{E}-05 \text { (strain amplitude, } \\
\mathrm{mm} / \mathrm{mm} \text { ) }\end{array}$ \\
\hline $\mathrm{c}_{2}$ & 3.66 (phase lag, radians) \\
\hline $\mathrm{d}_{2}$ & 0.05 \\
\hline
\end{tabular}

Similar to the stress data, the right-hand side of Table 6.7 shows the results of statistical analysis performed using TableCurve related to the accuracy of collected strain data. The left-hand side consists of quantitative information related to the strain amplitude referred to as " $\mathrm{b}_{2}$ " and the phase lag in the signal referred to as " $\mathrm{c}_{2}$ ". The overall output of data processing performed using "TableCurve" on data acquired for stress and strain cycles is shown in Table 6.8. Results of loading frequencies, other than the $20 \mathrm{~Hz}$, used in loading the sample in this example are also included. Figure 6.1 shows a plot of stress and strain values presented in Tables 6.5 and 6.6.

Table 6.8. Stress and strain amplitudes and phase lag calculated by "TableCurve"

\begin{tabular}{|c|c|c|c|c|}
\hline $\begin{array}{c}\text { Frequency } \\
(\mathrm{Hz})\end{array}$ & $\begin{array}{c}\text { Stress amplitude } \\
\mathrm{b}_{1}[\mathrm{MPa}]\end{array}$ & $\begin{array}{c}\text { Phase lag of stress } \\
\text { signal } \mathrm{c}_{1}[\mathrm{rad}]\end{array}$ & $\begin{array}{c}\text { Strain amplitude } \\
\mathrm{b}_{2}[\mu \varepsilon]\end{array}$ & $\begin{array}{c}\text { Phase lag of strain } \\
\text { signal } \mathrm{c}_{2}[\mathrm{rad}]\end{array}$ \\
\hline 20 & 1.091 & 3.959 & 99 & 3.663 \\
\hline 10 & 0.938 & 4.85 & 100 & 4.51 \\
\hline 5 & 0.822 & 5.198 & 100 & 4.823 \\
\hline 1 & 0.582 & 5.101 & 101 & 4.654 \\
\hline 0.3 & 0.427 & 5.148 & 100 & 4.639 \\
\hline 0.1 & 0.312 & 5.282 & 100 & 4.724 \\
\hline
\end{tabular}




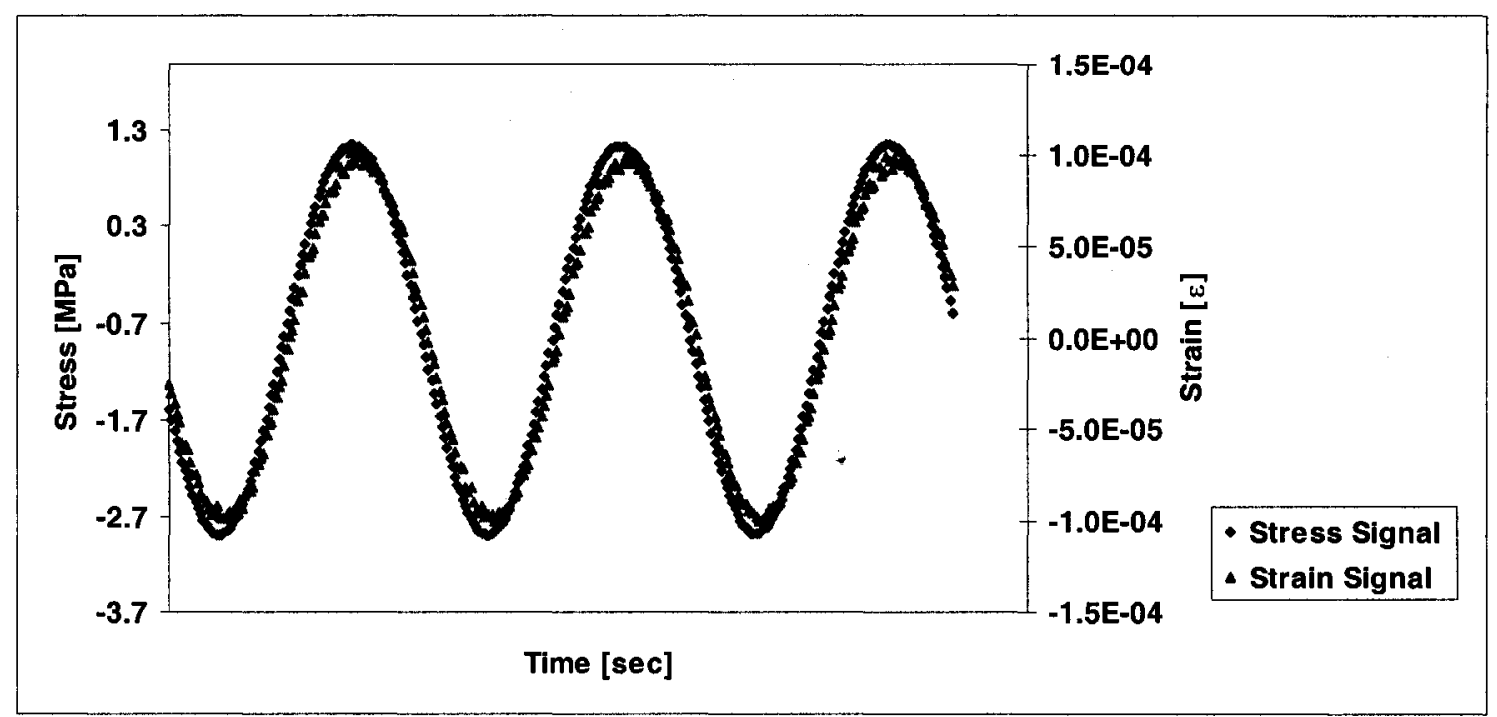

Figure 6.1. Stress and strain signals versus time measured at $20 \mathrm{~Hz}, 0^{\circ} \mathrm{C}$

\subsection{Assessment of AC Response to Different Factors}

The mechanistic properties of $\mathrm{AC}$ materials determined for all mix types were used to evaluate the response of $\mathrm{AC}$ mixtures to different factors. The following paragraphs discuss the influence of the elements used in the assessment including those shown in Table 6.1.

\subsubsection{Impact of Traffic Characteristics and Temperature Variations}

Asphalt concrete test results in this thesis are always described along with two of the important conditions at which the stiffness was measured, namely loading frequency and test temperature. Because of the nature of the material, it will be an inaccurate representation of the state at which the response was quantified if the stiffness is described based on one factor without including the other. Accordingly, the influence of these two factors on the mechanistic response of $\mathrm{AC}$ materials is analyzed in this subsection making specific reference to the loading frequency and test temperature condition. 
The loading frequency represents an important traffic characteristic, namely vehicle speed, which is known to influence the performance of roads. In general, lower speeds cause more damage as is the case with urban streets compared with highways. To examine the combined effect of loading frequency and test temperature on the $\mathrm{AC}$ response, data from the complex modulus tests performed in this study and reduced according to the steps discussed above, were used in the analysis. The dynamic modulus and phase angles determined from tests performed on an HMA 1 mix prepared using a PG 58-22 binder are shown in Tables 6.9 and 6.10 respectively.

Table 6.9. Dynamic modulus (MPa) determined at different frequencies and temperatures

\begin{tabular}{|c|c|c|c|c|c|}
\hline \multirow{2}{*}{$\begin{array}{c}\text { Frequency } \\
{[\mathrm{Hz}]}\end{array}$} & -10 & 0 & 20 & 30 & 40 \\
\hline 20 & 13300 & 10000 & 7850 & 2480 & 825 \\
\hline 10 & 12900 & 9540 & 6970 & 1890 & 608 \\
\hline 5 & 11900 & 8830 & 6220 & 1460 & 467 \\
\hline 1 & 11000 & 7940 & 4820 & 817 & 269 \\
\hline 0.3 & 10300 & 7040 & 3840 & 533 & 200 \\
\hline 0.1 & 9600 & 6280 & 3070 & 375 & 167 \\
\hline
\end{tabular}

Table 6.10. Phase angles $\left({ }^{\circ}\right)$ determined at different frequencies and temperatures

\begin{tabular}{|c|c|c|c|c|c|}
\hline $\begin{array}{c}\text { Frequency } \\
{[\mathrm{Hz}]}\end{array}$ & \multicolumn{5}{|c|}{ Temperature $\left[{ }^{\circ} \mathrm{C}\right]$} \\
\cline { 2 - 6 } & -10 & 0 & 20 & 30 & 40 \\
\hline 20 & 3.3 & 7.6 & 12.3 & 34.0 & 43.0 \\
\hline 10 & 3.5 & 7.7 & 13.5 & 36.8 & 42.6 \\
\hline 5 & 4.3 & 8.7 & 15.6 & 38.3 & 40.7 \\
\hline 1 & 5.8 & 10.1 & 20.1 & 40.4 & 36.4 \\
\hline 0.3 & 6.6 & 13.1 & 23.8 & 40.8 & 31.4 \\
\hline 0.1 & 9.5 & 16.2 & 27.5 & 37.6 & 26.5 \\
\hline
\end{tabular}

The dynamic moduli and phase angles were plotted against loading frequencies and test temperatures to evaluate the ability of the proposed characterization technique in capturing traffic and temperature impact on the mechanistic response of $\mathrm{AC}$ materials. Data shown in Tables 6.9 and 6.10 were plotted in Figures 6.2 to 6.5. 
First, the sensitivity of the measured phase angles to loading frequency was examined by plotting data from Table 6.10 as shown in Figures 6.2 and 6.3. The value of the phase angle decreased slightly with increased frequency at relatively low temperatures $(-10,0$, and $+20^{\circ} \mathrm{C}$ ) as shown in Figure 6.2. However, at warm temperatures (above $+25^{\circ} \mathrm{C}$ ), the phase angle increased with increased frequency. These two trends highlighted AC mix behaviour associated with rheological changes in the binder influenced by temperature changes where the response switches from relatively elastic at low temperatures to viscoelastic at warm temperatures.

Figure 6.3 shows that above $+30^{\circ} \mathrm{C}$ the phase angle reverses its trend and starts to decrease with an increase in the temperature. This phenomenon, addressed in the literature, occurs when the binder loses stiffness at relatively high temperatures where aggregate contribution predominates in the response of the mixture. The binder effect tends to be negligible and hence the phase angle is expected to drop after $+30^{\circ} \mathrm{C}$.

Figure 6.4 reflects sensitivity of the dynamic modulus to loading frequency. The dynamic modulus increased with the increase in loading frequency according to test results obtained at all temperatures. As expected, the dynamic modulus decreased with increasing temperatures for all loading frequencies as shown in Figure 6.5.

Results from tests performed on an HMA 1 mix prepared using a PG 58-22 binder are used here to reflect the influence of frequency on phase angles and dynamic modulus of AC mixes as shown in Figures 6.2 and 6.4 respectively. However, this phenomenon has been observed in the results obtained from testing of all mix types used in this investigation. 


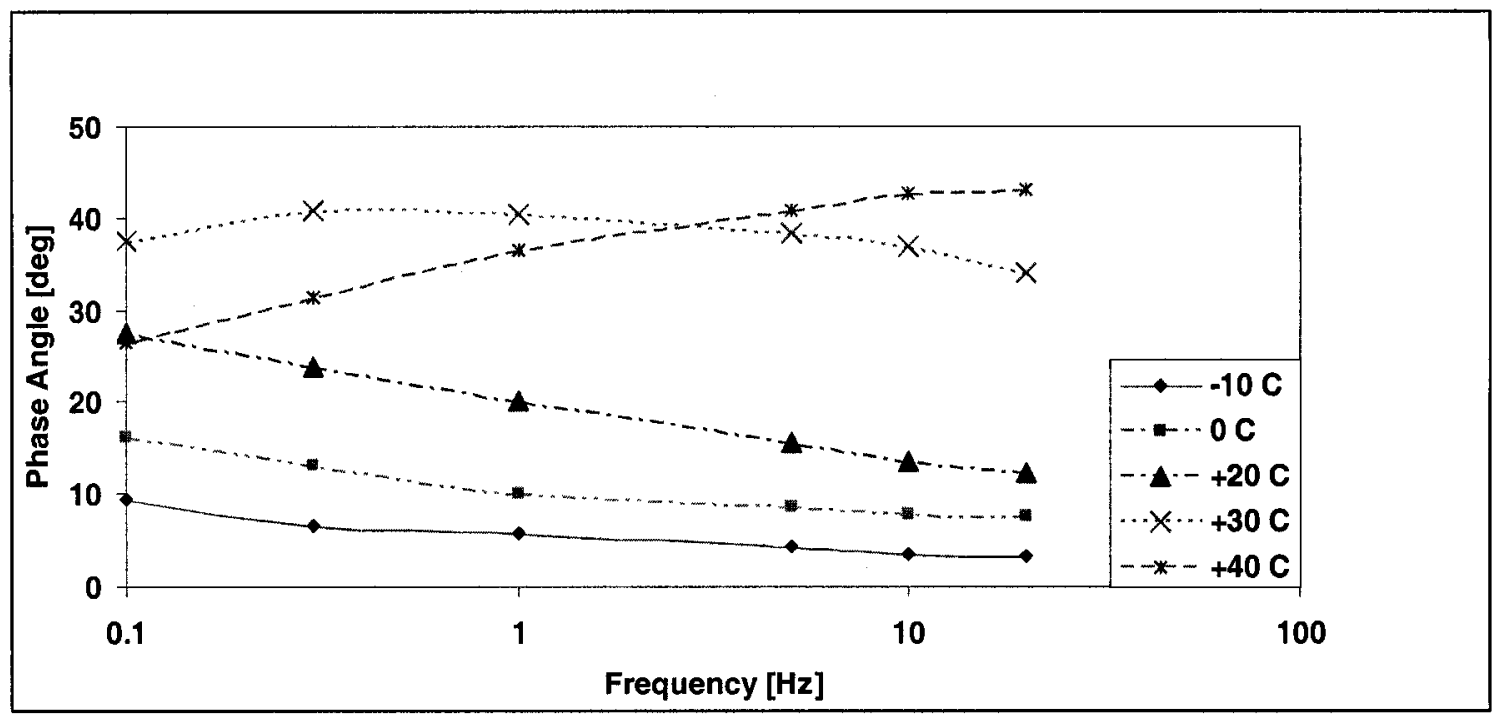

Figure 6.2. Effect of frequency on phase angle [HMA 1, PG 58-22]

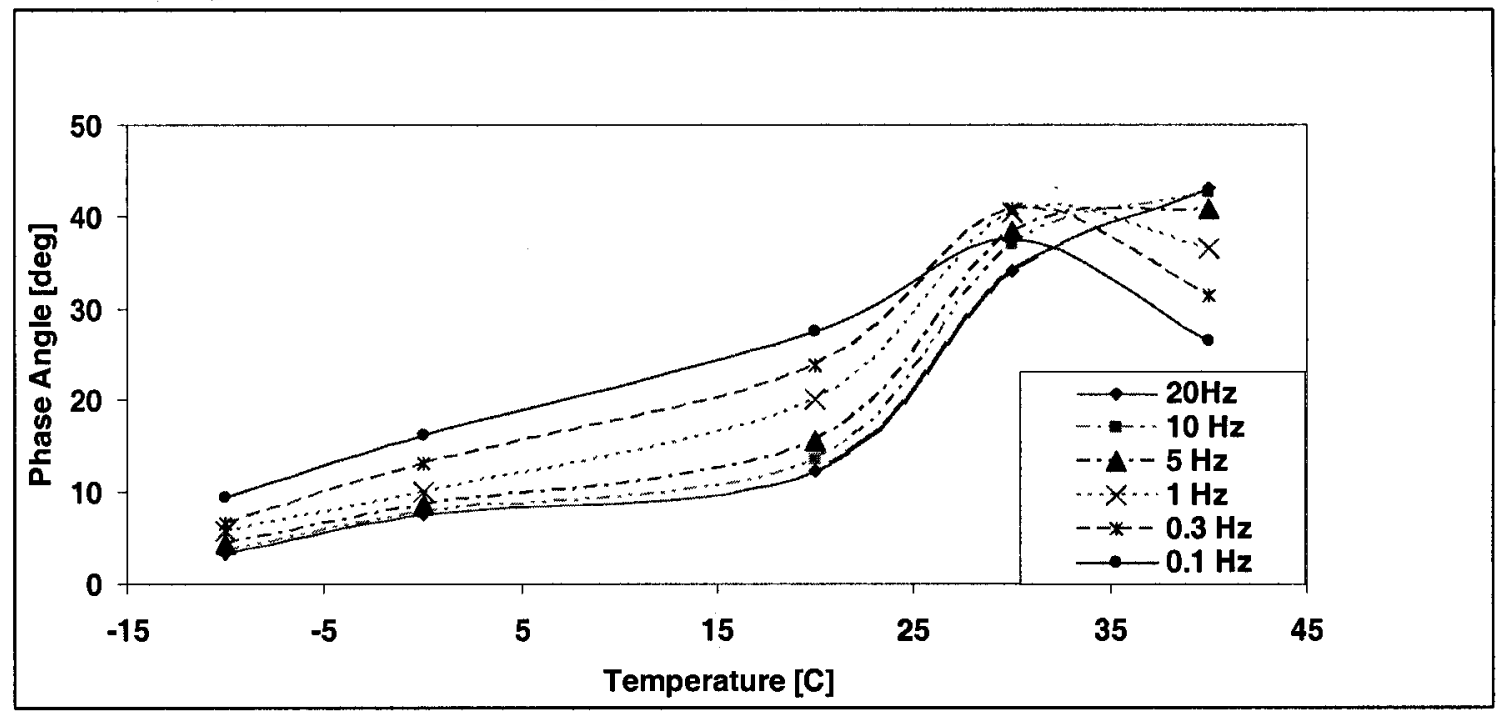

Figure 6.3. Effect of testing temperature on phase angle [HMA 1, PG 58-22] 


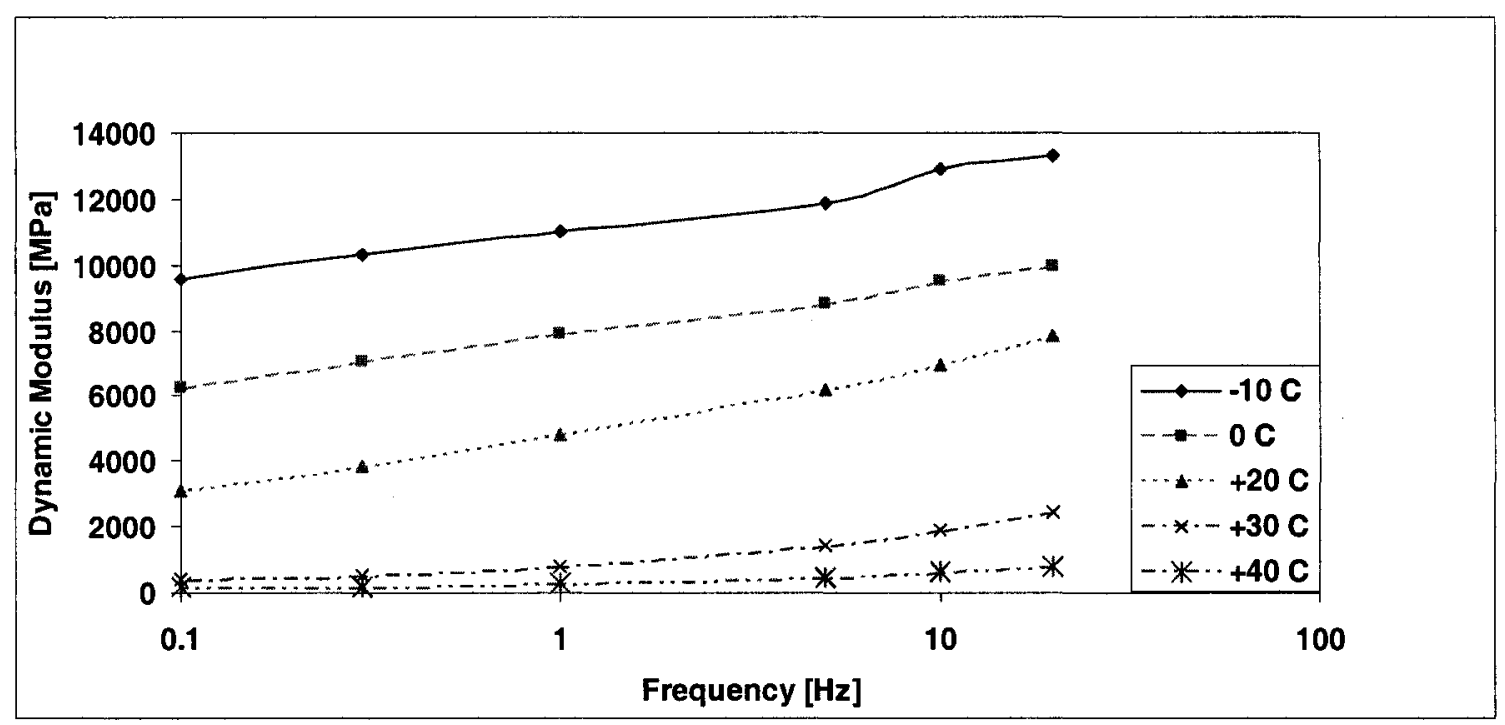

Figure 6.4. Effect of loading frequency on dynamic modulus [HMA 1, PG 58-22]

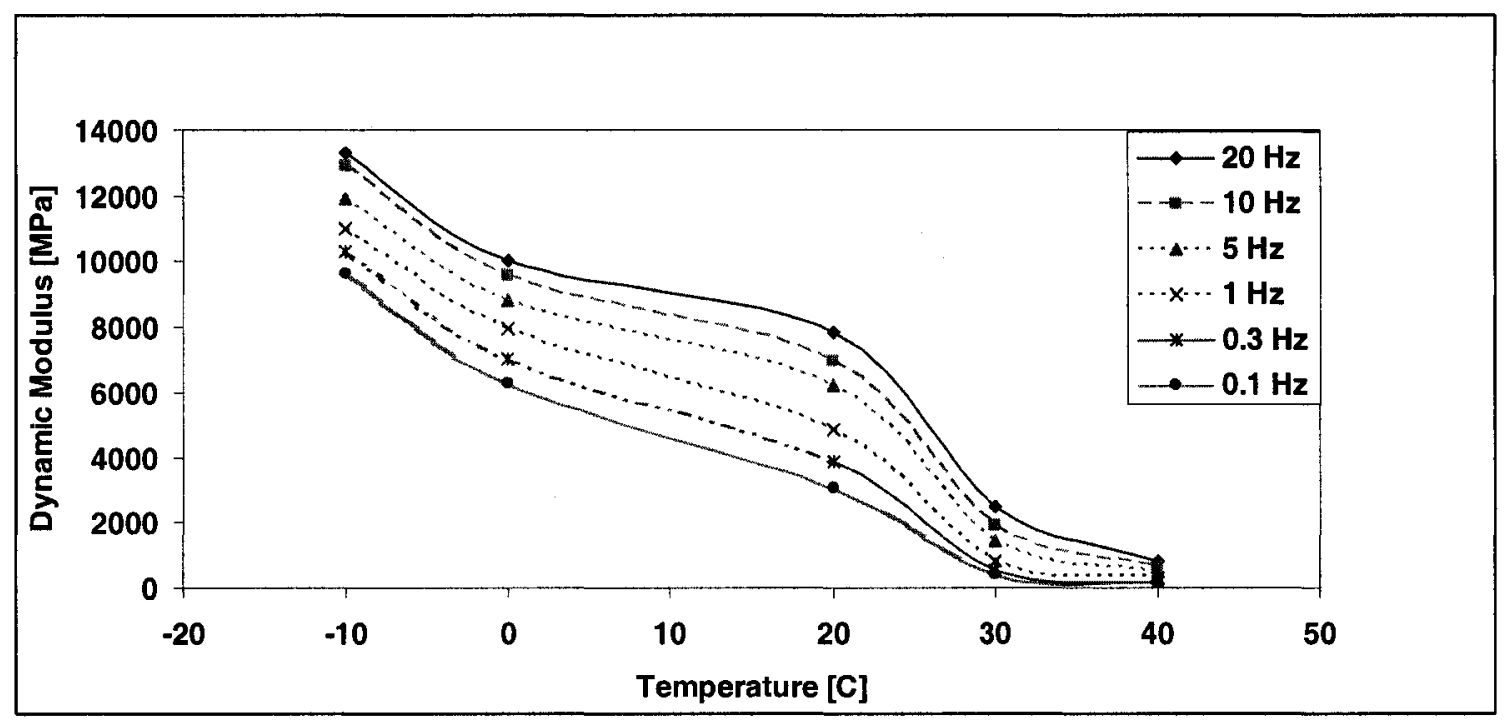

Figure 6.5. Effect of testing temperature on dynamic modulus [HMA 1, PG 58-22]

\subsubsection{Response by Different Mix Types}

The analysis discussed in the previous subsection was applied to all other mixes using different binder combinations. The mechanical properties determined for the two uniquely different mix types HMA 1 and HMA 3, prepared with a PG 58-22 binder, are discussed first to demonstrate the effectiveness of the proposed characterization 
technique in distinguishing between two different mixes using the same binder. The HMA 3 is a typical dense graded surface mix with a relatively high binder content compared with the coarse graded HMA 1 mix commonly used as a stabilized base. The physical differences, presented earlier (see Table 4.11, Chapter 4), influenced the mechanistic response of these mixes as reflected in the analysis performed using the complex modulus parameters determined using the proposed testing protocol. The impact of the mix type on the dynamic modulus was evaluated first at two temperatures with varying frequencies. Secondly, the dynamic modulus data generated using two loading frequencies was evaluated at a number of test temperatures. The results representing these two data formats are shown in Tables 6.11 and 6.12.

Table 6.11. Dynamic modulus determined at a constant temperature and different loading frequencies [HMA, PG 58-22]

\begin{tabular}{|c|c|c|c|c|}
\hline \multirow{3}{*}{$\begin{array}{c}\text { Frequency } \\
{[\mathrm{Hz}]}\end{array}$} & \multirow{2}{*}{\multicolumn{2}{|c|}{$\begin{array}{c}\text { (A) Dynamic Modulus [MPa], } \\
0^{\circ} \mathrm{C} \\
\text { Mix Type } \\
\end{array}$}} & \multirow{2}{*}{\multicolumn{2}{|c|}{$\begin{array}{c}\text { (B) Dynamic Modulus [MPa], } \\
+40^{\circ} \mathrm{C} \\
\text { Mix Type }\end{array}$}} \\
\hline & & & & \\
\hline & HMA 1 & HMA 3 & HMA 1 & HMA 3 \\
\hline 20 & $1.0 \mathrm{E}+04$ & $2.1 \mathrm{E}+04$ & $2.5 \mathrm{E}+03$ & $3.2 \mathrm{E}+03$ \\
\hline 10 & $9.5 \mathrm{E}+03$ & $2.0 \mathrm{E}+04$ & $1.9 \mathrm{E}+03$ & $2.3 \mathrm{E}+03$ \\
\hline 5 & $8.8 \mathrm{E}+03$ & $1.8 \mathrm{E}+04$ & $1.5 \mathrm{E}+03$ & $1.7 \mathrm{E}+03$ \\
\hline 1 & $7.9 \mathrm{E}+03$ & $1.5 \mathrm{E}+04$ & $8.2 \mathrm{E}+02$ & $8.7 \mathrm{E}+02$ \\
\hline 0.3 & $7.0 \mathrm{E}+03$ & $1.3 \mathrm{E}+04$ & $5.3 \mathrm{E}+02$ & $5.3 \mathrm{E}+02$ \\
\hline 0.1 & $6.3 \mathrm{E}+03$ & $1.1 \mathrm{E}+04$ & $3.8 \mathrm{E}+02$ & $3.4 \mathrm{E}+02$ \\
\hline
\end{tabular}

Table 6.12. Dynamic modulus determined at a constant loading frequency and different temperature [HMA, PG 58-22]

\begin{tabular}{|c|c|c|c|c|}
\hline \multirow{3}{*}{$\begin{array}{c}\text { Temperature } \\
{\left[{ }^{\circ} \mathrm{C}\right]}\end{array}$} & \multirow{2}{*}{\multicolumn{2}{|c|}{$\begin{array}{c}\text { (A) Dynamic Modulus [MPa], } \\
20 \mathrm{~Hz} \\
\text { Mix Type }\end{array}$}} & \multirow{2}{*}{\multicolumn{2}{|c|}{$\begin{array}{c}\text { (B) Dynamic Modulus [MPa], } \\
0.3 \mathrm{~Hz} \\
\text { Mix Type }\end{array}$}} \\
\hline & & & & \\
\hline & HMA 1 & HMA 3 & HMA 1 & HMA 3 \\
\hline-10 & $1.3 \mathrm{E}+04$ & $2.4 \mathrm{E}+04$ & - & - \\
\hline 0 & $1.0 \mathrm{E}+04$ & $2.1 \mathrm{E}+04$ & $7.0 \mathrm{E}+03$ & $1.3 \mathrm{E}+04$ \\
\hline 20 & $7.8 \mathrm{E}+03$ & $1.5 \mathrm{E}+04$ & $3.8 \mathrm{E}+03$ & $6.8 \mathrm{E}+03$ \\
\hline 30 & $2.5 \mathrm{E}+03$ & $3.2 \mathrm{E}+03$ & $5.3 \mathrm{E}+02$ & $5.3 \mathrm{E}+02$ \\
\hline 40 & $8.3 \mathrm{E}+02$ & $1.2 \mathrm{E}+03$ & $2.0 \mathrm{E}+02$ & $1.8 \mathrm{E}+02$ \\
\hline
\end{tabular}


Dynamic modulus values shown in Tables 6.11 were plotted against frequencies at 0 and $+40^{\circ} \mathrm{C}$ as shown in Figures 6.6 and 6.7 respectively. The difference between the dynamic modulus of the HMA 1 and HMA 3 mixes is apparent across the whole frequency sweep at both test temperatures of $0^{\circ} \mathrm{C}$ (as shown in Figures 6.6) and $40^{\circ} \mathrm{C}$ (as shown in Figure 6.7). The stiffness of the HMA 1 at $0^{\circ} \mathrm{C}$ was lower than that of the HMA 3 (a maximum difference of 120\%). The high binder content used in the HMA 3 mix dominated the response of the mix exposed to such a low test temperature where the binder becomes very brittle. This analysis is supported by the results plotted in Figure 6.7 for tests performed at $+40^{\circ} \mathrm{C}$ where the difference between the two mixes diminished substantially. At such a warm temperature, the aggregate skeleton governs the overall response. The slight difference determined at the high loading frequency of $10 \mathrm{~Hz}$ may be explained by the fact that the HMA 3 mix was denser.

The test data in Table 6.12, plotted to show the dynamic modulus-temperature relationship (see Figures 6.8 and 6.9), support the above discussions. It is clear that the HMA 3 is stiffer than HMA 1 at cold temperatures; meanwhile, the two mixes demonstrated almost identical values of complex modulus at warm temperatures. 


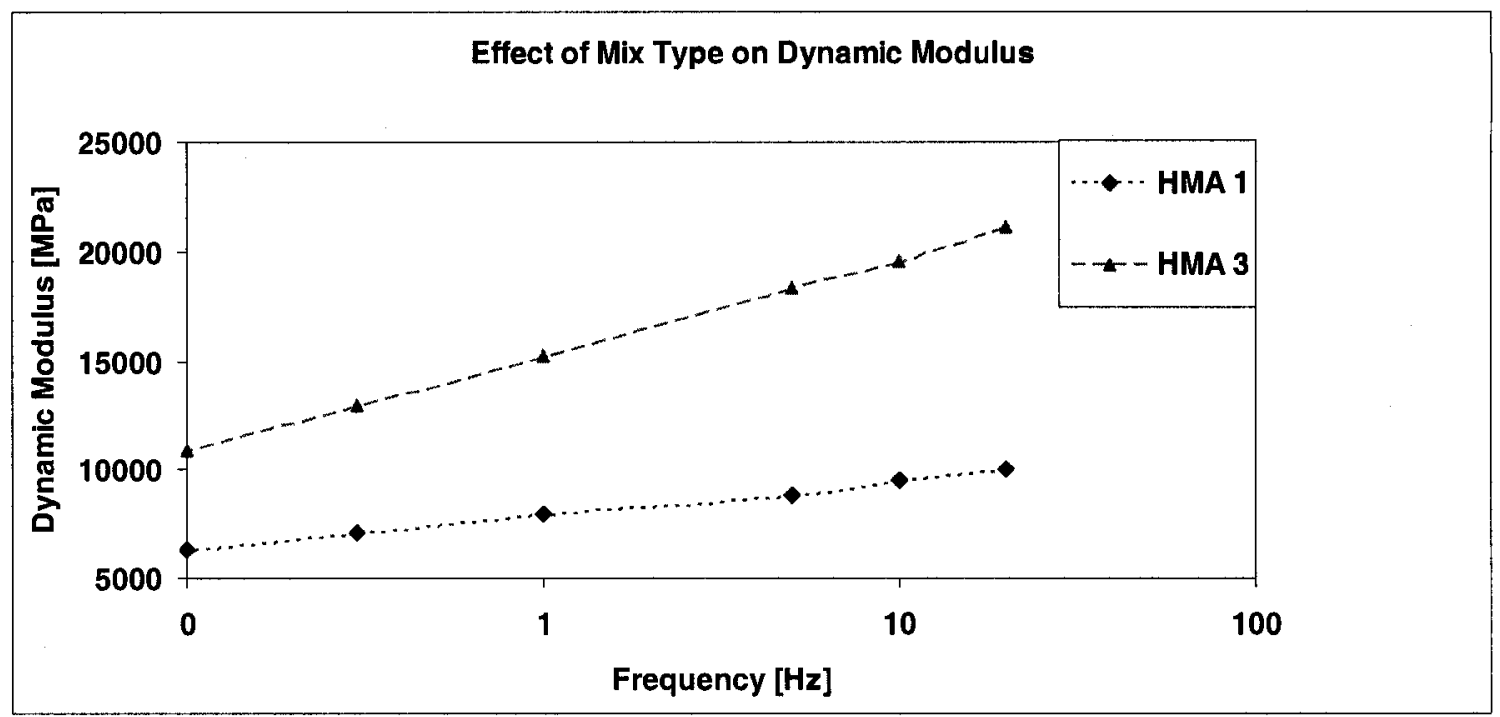

Figure 6.6. The dynamic modulus determined at different loading frequencies at $0^{\circ} \mathrm{C}$

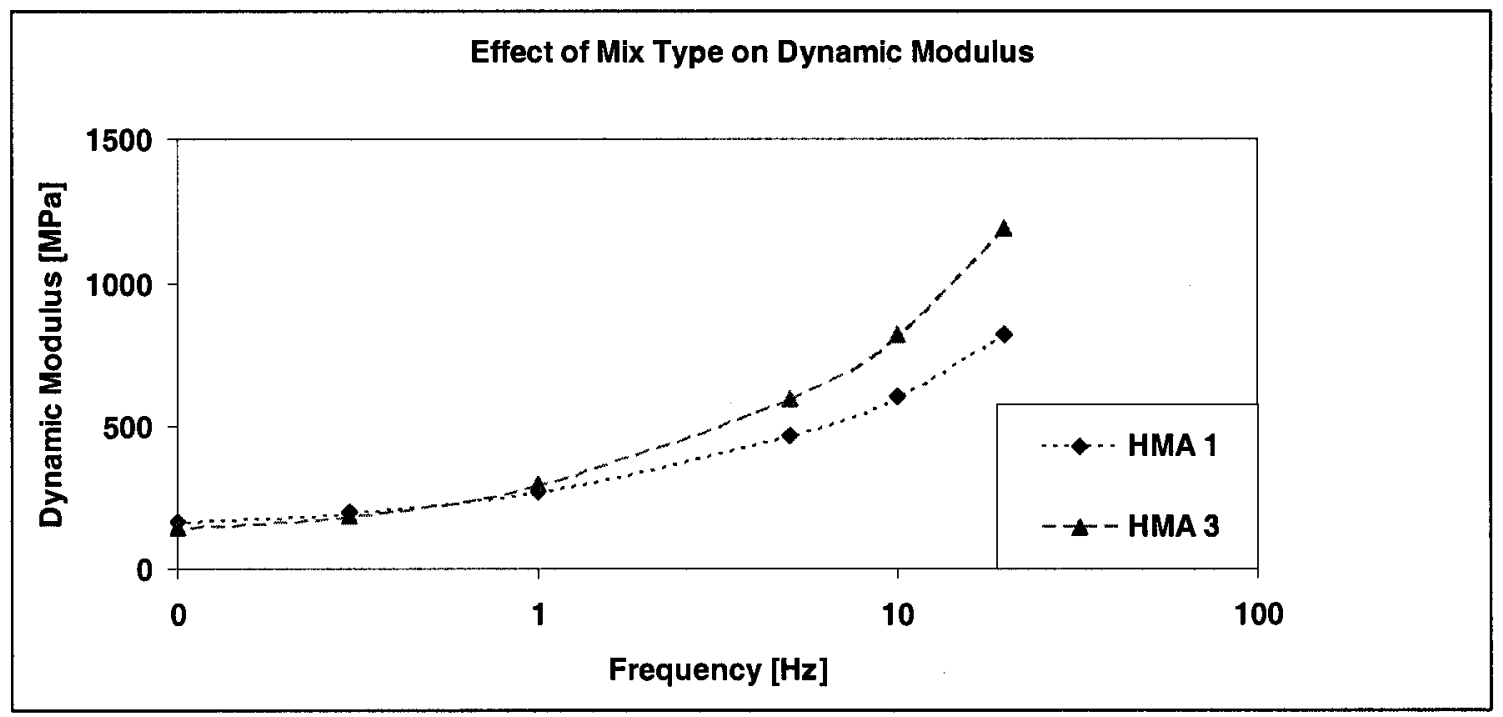

Figure 6.7. The dynamic modulus determined at different loading frequencies at testing temperature of $+40^{\circ} \mathrm{C}$ 


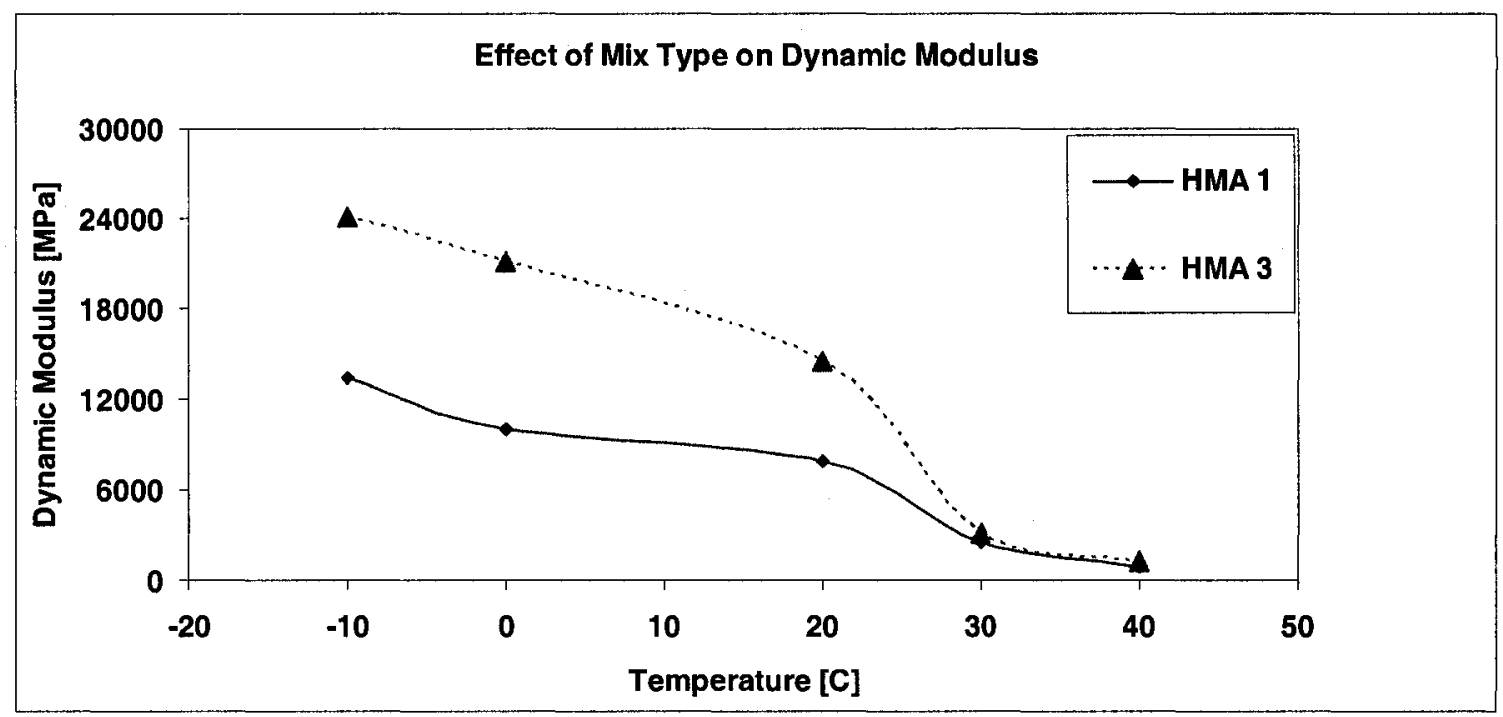

Figure 6.8. The dynamic modulus determined at different test temperatures and a loading frequency of $20 \mathrm{~Hz}$

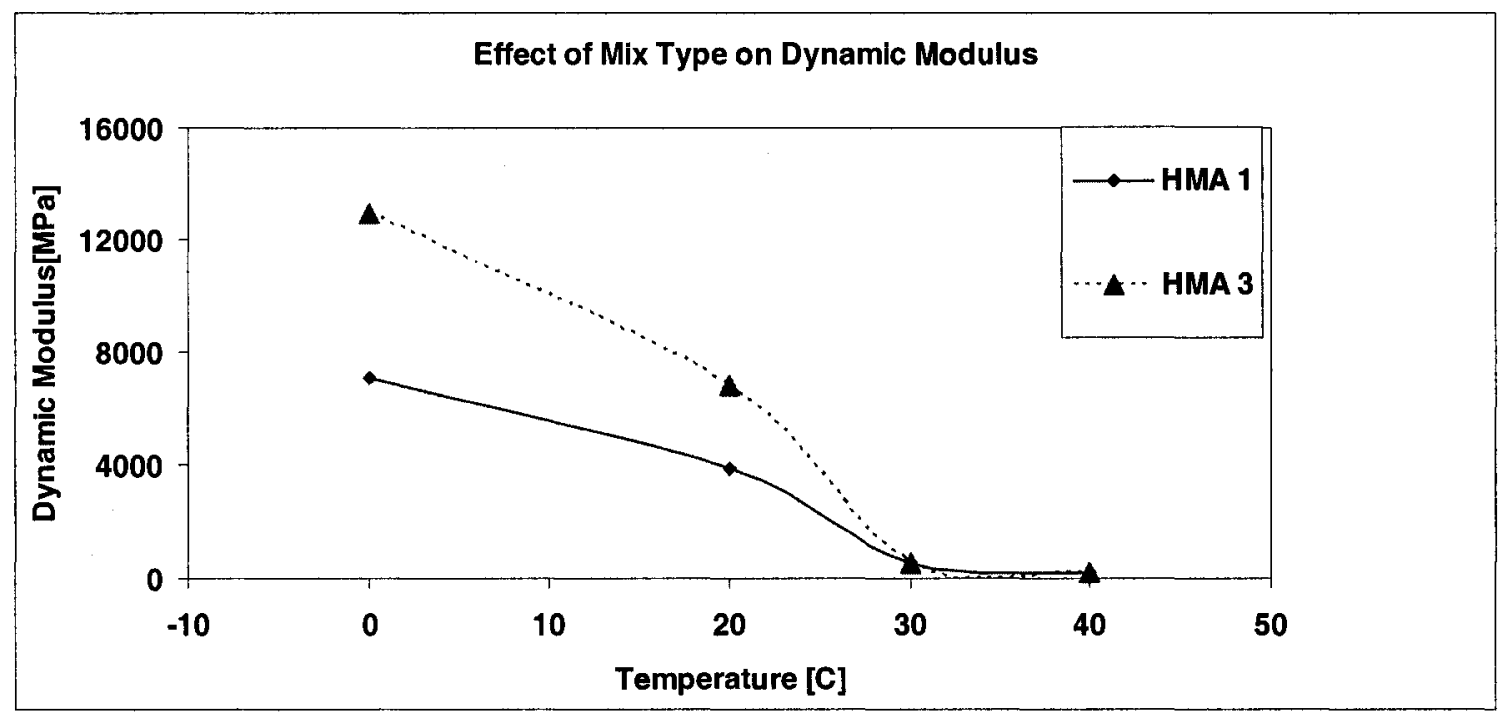

Figure 6.9. The dynamic modulus determined at different test temperatures and a loading frequency of $0.3 \mathrm{~Hz}$

\subsubsection{Effect of Binder Type}

Analyzing the impact of binders on the dynamic modulus necessitates taking into consideration the binder's viscosity, which is defined as "the property of the asphalt cement that determines its ability to resist flow." Accordingly, the more viscous a binder is, the more it will resist flow and conversely the less viscous a binder is, the less it will 
resist flow. Also important is the fact that engineered binders, which target a specific function for a specific application, must be considered in the analysis of the determined mechanistic properties.

Discussions related to the impact of the binder type on the dynamic modulus and phase angle involve analysis of data collected for the same mix prepared with the three different binders used in this thesis. Mechanistic properties of a Marshall mix (HMA 3) were used to conduct the investigation using the results shown in Tables 6.13 and 6.14. An evaluation of the effect of the binder type on the dynamic modulus was performed at two unique loading frequencies $(1$ and $20 \mathrm{~Hz})$ at varying temperatures as shown in Table 6.13. The phase angle determined at different temperatures at $20 \mathrm{~Hz}$ loading frequency is shown in Table 6.14.

Table 6.13. Dynamic modulus determined at different Temperatures (Loading frequency of 20 and $1 \mathrm{~Hz}$ )

\begin{tabular}{|c|c|c|c|c|c|c|}
\hline \multirow{3}{*}{$\begin{array}{c}\text { Temperature } \\
{\left[{ }^{\circ} \mathrm{C}\right]}\end{array}$} & \multicolumn{3}{|c|}{ Dynamic Modulus [MPa], $20 \mathrm{~Hz}$} & \multirow{2}{*}{\multicolumn{3}{|c|}{$\frac{\text { Dynamic Modulus [MPa], } 1 \mathrm{~Hz}}{\text { Binder Type }}$}} \\
\hline & \multicolumn{3}{|c|}{ Binder Type } & & & \\
\hline & PG 64-32 & G 58-22 & PG 52-34 & PG 64-34 & PG 58-22 & PG 52-34 \\
\hline 0 & 10116 & 15455 & 12980 & 7020 & 11240 & 6188 \\
\hline 20 & 4730 & 7100 & 2840 & 2510 & 3137 & 871 \\
\hline 30 & 2130 & 3580 & 960 & 941 & 1147 & 248 \\
\hline 40 & 1061 & 1667 & 358 & 480 & 485 & 120 \\
\hline
\end{tabular}

Table 6.14. Phase angle determined at different Temperatures (Loading frequency of 20 and $1 \mathrm{~Hz}$ )

\begin{tabular}{|c|c|c|c|c|c|c|c|}
\hline \multirow{2}{*}{$\begin{array}{c}\text { Temperature } \\
{\left[{ }^{\circ} \mathrm{C}\right]}\end{array}$} & \multicolumn{3}{|c|}{ Phase Angle $\left[{ }^{\circ}\right], 20 \mathrm{~Hz}$} & \multicolumn{3}{c|}{ Phase Angle $\left[{ }^{\circ}\right], 1 \mathrm{~Hz}$} \\
\hline & \multicolumn{3}{|c|}{ Binder Type } & & \multicolumn{3}{c|}{ Binder Type } \\
\hline-10 & PG 64-34 & PG 58-22 & PG 52-34 & & PG 64-34 & PG 58-22 & PG 52-34 \\
\hline 0 & 11.3 & 7.1 & 5.4 & & 10.6 & 7.3 & 10.4 \\
\hline 20 & 28.5 & 20.7 & 38.2 & & 17.4 & 12.3 & 22.9 \\
\hline 30 & 39.4 & 32.2 & 44.7 & & 32.6 & 31.2 & 41.5 \\
\hline 40 & 39.2 & 40.8 & 42.8 & & 27.9 & 41.9 & 42.8 \\
\hline
\end{tabular}


The determined dynamic moduli and phase angles of the mixes shown in Tables 6.13 and 6.14 were plotted in Figures 6.10 to 6.12 . Figure 6.10 reflects a clear difference at low temperatures in dynamic modulus values measured for the HMA 3 mix prepared using PG 64-34 and PG 58-22 binders. The dynamic modulus determined for samples prepared with the engineered binder (PG 64-34) increased slightly with the drop in temperature but remained far below from that of the neat binder (PG 58-22). The binder engineering process successfully lowered the stiffness (by 53\%) compared with the neat binder, which seems to explain the switch to this engineered binder in cold regions such as Ottawa-Carleton in order to reduce the incidence of low-temperature cracking. Practicing engineers make reference to the rubbery nature of the PG 64-34 binder and believe it resists thermal cracking. The results for the two binders reflect no difference at warm temperatures since the aggregate skeleton dominates the response at such temperature conditions (between +30 and $+40^{\circ} \mathrm{C}$ ).

When binders with similar low-temperature grades were compared using dynamic modulus values determined at different temperatures (PG 52-34 and 64-34), the results shown in Figure 6.11 reflected much less difference between the two binders. Binder treatment intended for achieving stability at high temperatures (PG 64-34) improved lowtemperature performance slightly. Similar to the previous situation, the plotted dynamic modulus values within the warm temperature range showed no difference between the two binders since it is the range where the aggregate skeleton dominates the response.

On the other hand, the phase angle data presented in Table 6.14 were plotted in Figures 6.12 and 6.13. Figure 6.12 suggests that there is little difference in the phase angle determined for the mix prepared with PG 64-34 and 52-34. The phase angle determined 
at low temperatures was very small, which is in agreement with the assumption that the response may be considered elastic. The phase angle determined for the mix prepared with the PG 64-34 binder was 54\% less than that determined for the PG 58-22 binder at high temperatures and low loading frequency $(1 \mathrm{~Hz})$, as shown in Figure 6.13. With the binder softening and the aggregate skeleton dominating the response at warm temperatures, the higher stability of the PG 64-34 binder was reflected by the test data.

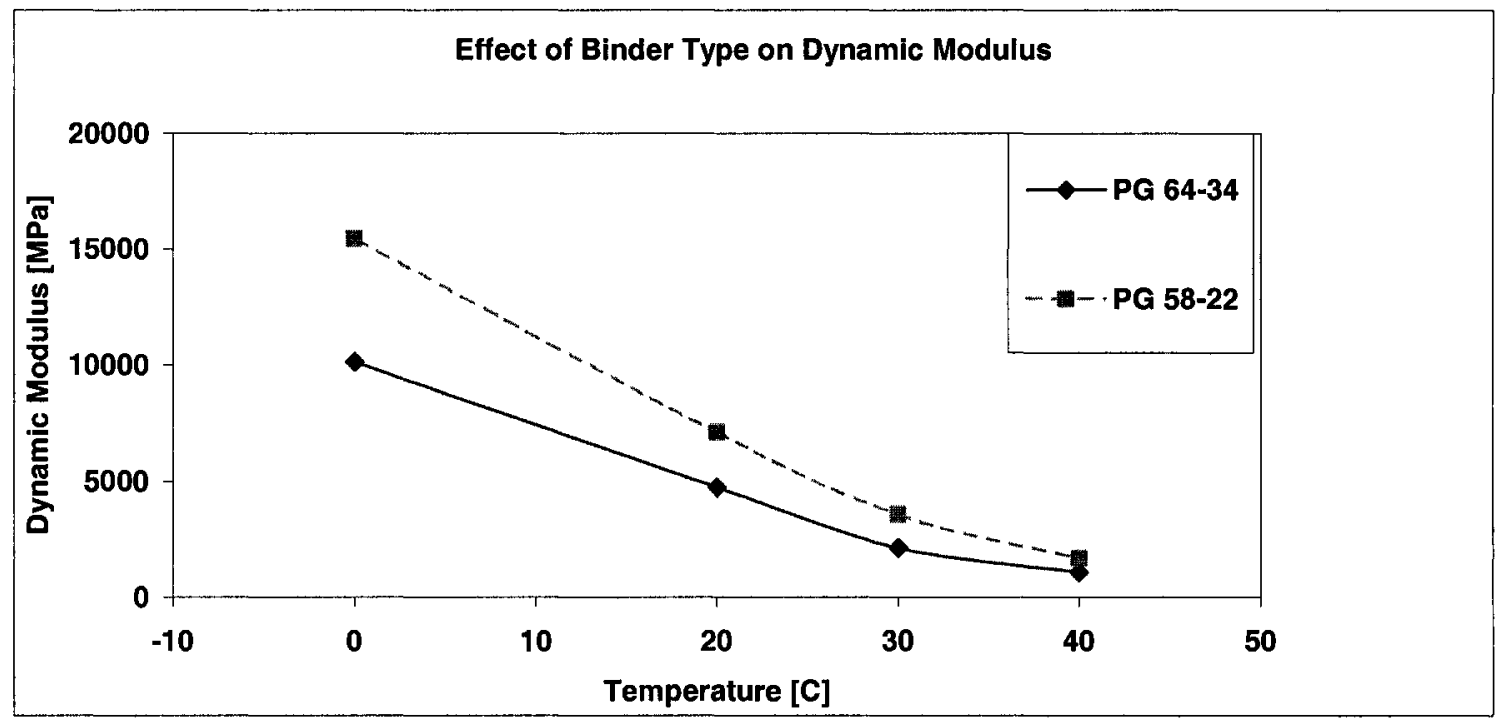

Figure 6.10. Dynamic modulus determined at different temperatures and a loading frequency of $20 \mathrm{~Hz}$

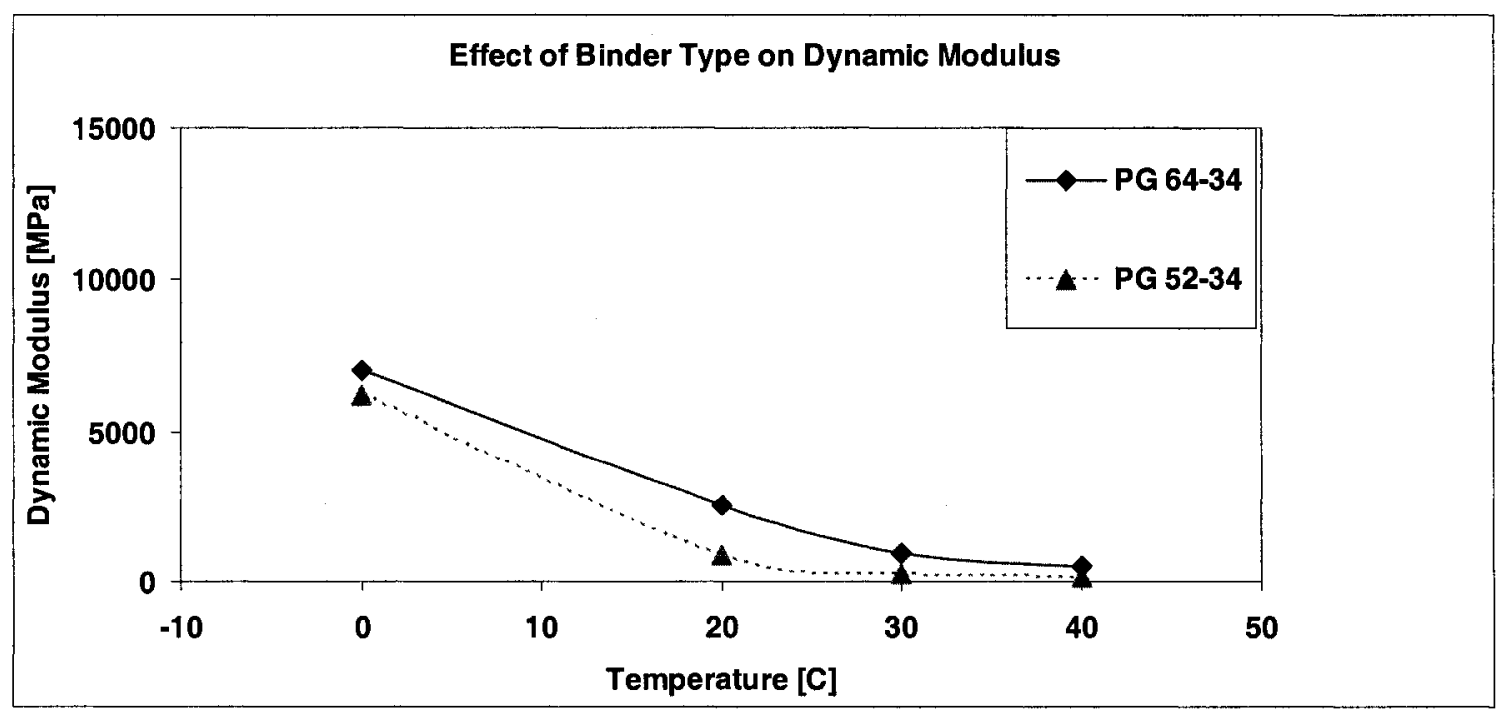

Figure 6.11. Dynamic modulus determined at different temperatures and a loading frequency of $1 \mathrm{~Hz}$ 


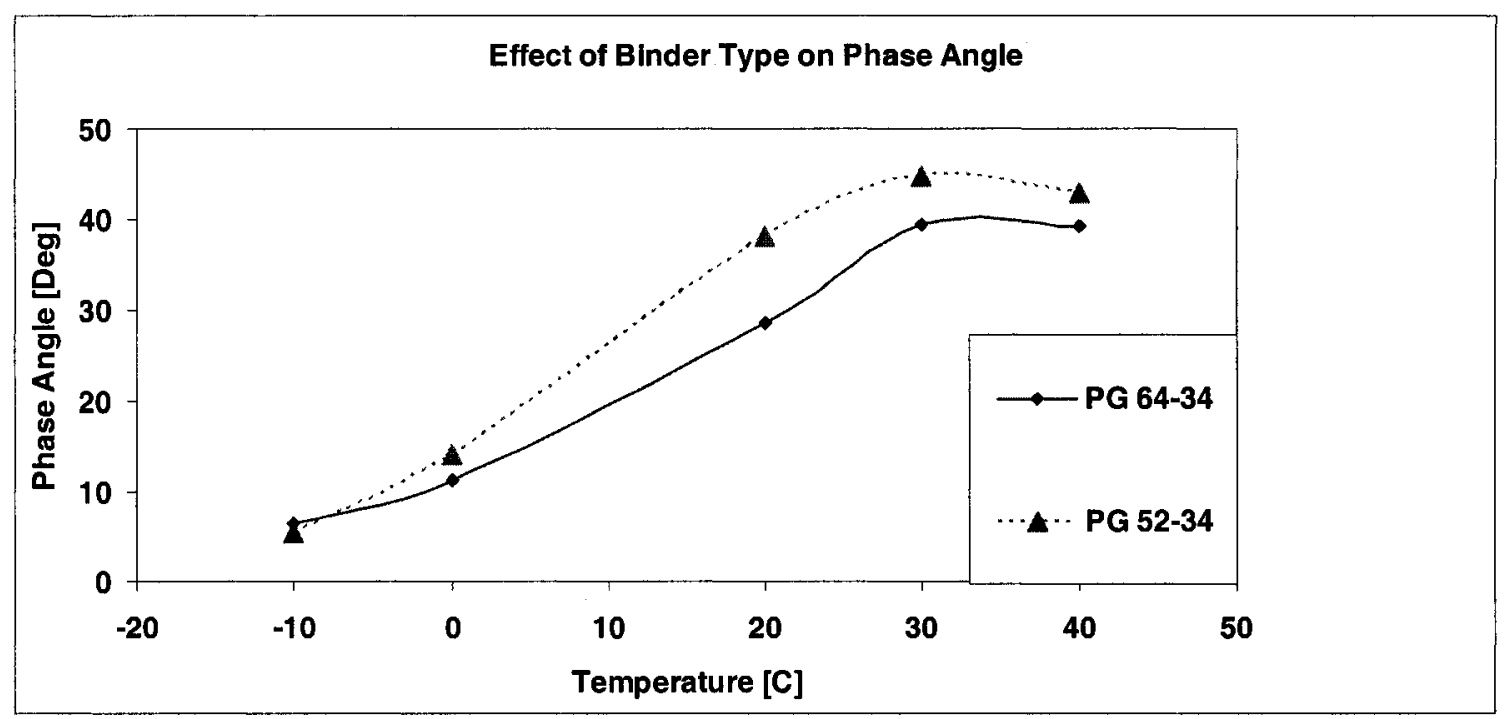

Figure 6.12. Phase angle determined at different temperatures and a loading frequency of $20 \mathrm{~Hz}$

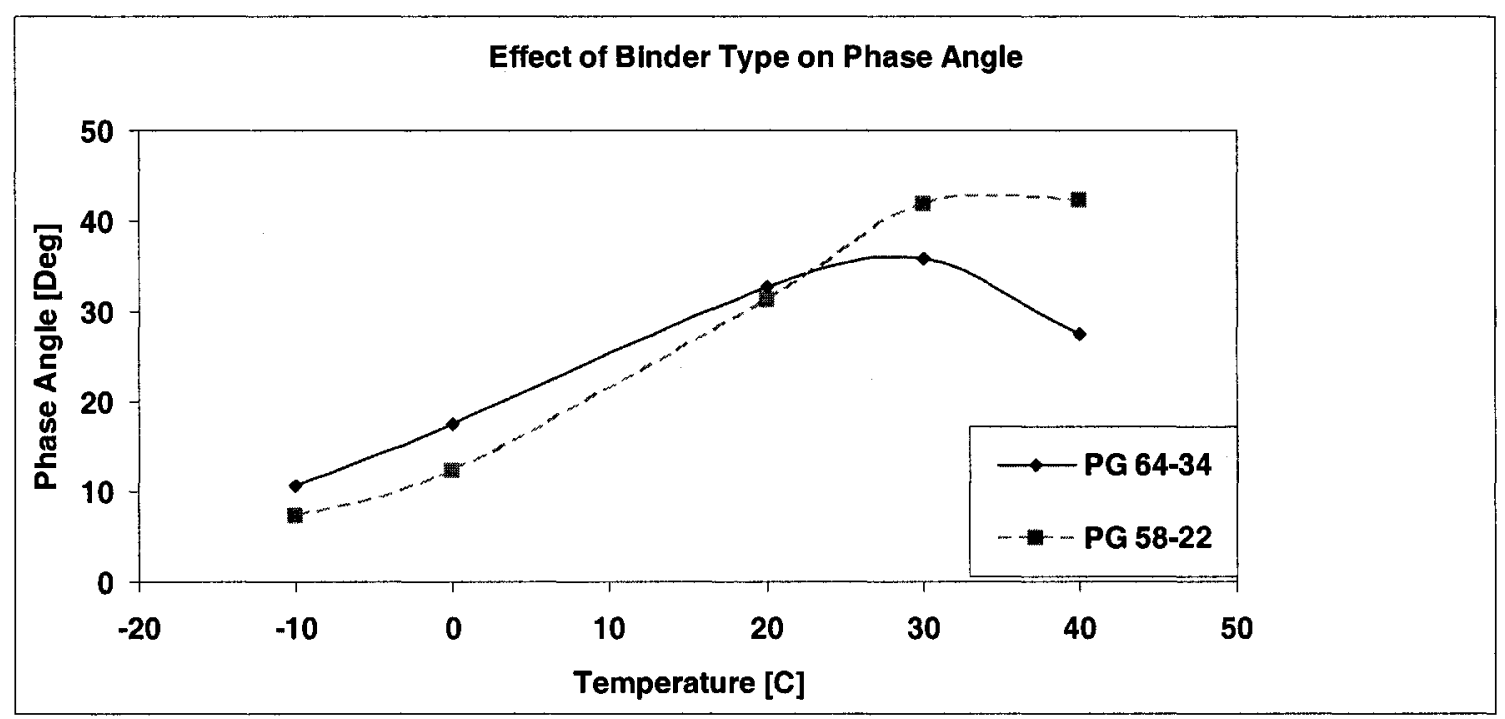

Figure 6.13. Phase angle determined at different temperatures and a loading frequency of $1 \mathrm{~Hz}$

It worth mentioning that the dynamic modulus and phase angle trends obtained for the HMA 3 mix prepared with different binder grades were confirmed in tests performed in all other tested mixes. 


\subsubsection{Construction Quality}

The quality of construction determines the microstructural features of the AC material. These features include particle distribution and orientation, which are influenced by the manufacturing process and compaction energy. It also includes the percentage of air voids in the mix. These features influence the mechanical behaviour of the compacted mix. As mentioned in Chapter 4, the evaluation of the impact of construction quality on the mechanistic response of $\mathrm{AC}$ materials performed in this study involved testing samples prepared with different percentages of air voids in the compacted mix. A SuperPave mix (SP 2) prepared with a PG 64-34 binder was used to prepare the samples compacted using different numbers of gyrations to produce different air voids contents. The dynamic modulus and phase angle, determined at two temperatures $\left(0\right.$ and $\left.+30^{\circ} \mathrm{C}\right)$ are shown in Tables 6.15 and 6.16.

The dynamic modulus and phase angle values shown in Tables 6.15 and 6.16 were plotted against air voids to evaluate the effect of different air voids content on the mechanistic response of the tested AC samples as shown in Figures 6.14 to 6.17.

Figure 6.14 shows that the dynamic modulus was influenced by the air voids content. Dynamic modulus values measured at a temperature of $0^{\circ} \mathrm{C}$ decreased as air voids increased for the entire frequency sweep. The dynamic modulus for the $7.1 \%$ air voids determined at a loading frequency of $20 \mathrm{~Hz}$ dropped by $41 \%$ compared with the $3.5 \%$ air void sample. A similar change was observed in a test performed at a warmer temperatures $\left(+30^{\circ} \mathrm{C}\right)$ and a loading frequency of $20 \mathrm{~Hz}$, where the dynamic modulus value at $6.0 \%$ air voids dropped by $43 \%$ compared with the sample with $3.5 \%$ air voids, as shown in Figure 6.15. 
Table 6.15. Dynamic modulus (MPa) determined at different air voids \% at 0 and $+300 C$ test temperatures

\begin{tabular}{|c|c|c|c|c|}
\hline \multirow{2}{*}{$\begin{array}{c}\text { Temperature } \\
{\left[{ }^{\circ} \mathrm{C}\right]}\end{array}$} & $\begin{array}{c}\text { Air Voids } \\
{[\%]}\end{array}$ & \multicolumn{3}{|c|}{ Frequency $[\mathrm{Hz}]$} \\
\cline { 2 - 5 } & 0 & 20 & 10 & 5 \\
\hline \multirow{5}{*}{0} & 3.5 & $1.8 \mathrm{E}+04$ & $1.5 \mathrm{E}+04$ & $1.4 \mathrm{E}+04$ \\
\cline { 2 - 6 } & 3.8 & $1.7 \mathrm{E}+04$ & $1.5 \mathrm{E}+04$ & $1.4 \mathrm{E}+04$ \\
\cline { 2 - 6 } & 6.0 & $1.4 \mathrm{E}+04$ & $1.3 \mathrm{E}+04$ & $9.3 \mathrm{E}+03$ \\
\cline { 2 - 6 } & 7.1 & $1.3 \mathrm{E}+04$ & $1.0 \mathrm{E}+04$ & $9.4 \mathrm{E}+03$ \\
\hline \multicolumn{5}{|c}{} \\
\hline \multirow{5}{*}{+30} & 3.5 & $2.7 \mathrm{E}+03$ & $2.2 \mathrm{E}+03$ & $1.8 \mathrm{E}+03$ \\
\hline & 6.0 & $2.2 \mathrm{E}+03$ & $1.6 \mathrm{E}+03$ & $1.3 \mathrm{E}+03$ \\
\cline { 2 - 6 } & 10.6 & $1.9 \mathrm{E}+03$ & $1.4 \mathrm{E}+03$ & $1.1 \mathrm{E}+03$ \\
\hline
\end{tabular}

Table 6.16. Phase angles determined at different air voids $\%$ at 0 and $+300 \mathrm{C}$ test temperatures

\begin{tabular}{|c|c|c|c|c|}
\hline Temperature & Air Voids & \multicolumn{3}{|c|}{ Frequency $[\mathrm{Hz}]$} \\
\cline { 3 - 5 }$\left[{ }^{\circ} \mathrm{C}\right]$ & {$[\%]$} & 20 & 10 & 5 \\
\hline \multirow{2}{*}{0} & 3.5 & 13.5 & 15.5 & 17.1 \\
\cline { 2 - 5 } & 6.0 & 11.3 & 13.2 & 14.5 \\
\cline { 2 - 5 } & 7.1 & 15.9 & 16.6 & 18.3 \\
\hline \multicolumn{3}{|c|}{} \\
\hline+30 & 3.5 & 28.8 & 28.2 & 27.8 \\
\cline { 2 - 5 } & 6.0 & 39.4 & 40.3 & 38.8 \\
\cline { 2 - 5 } & 10.6 & 40.1 & 40.8 & 39.1 \\
\cline { 2 - 5 } & \multicolumn{3}{|c}{}
\end{tabular}

The change in phase angle evaluated at $0^{\circ} \mathrm{C}$ was negligible, as shown in Figure 6.16. However, phase angles determined at $+30^{\circ} \mathrm{C}$ using a loading frequency of $20 \mathrm{~Hz}$ increased by $40 \%$ when the air voids percentage increased from $3.5 \%$ to $6.0 \%$, as shown in Figure 6.17. The phase angle remained the same with air voids changing from $6 \%$ to $11 \%$, suggesting that the integrity of the mix reached a low state at $6 \%$ air voids and beyond. When comparing the test results obtained at 0 and $30^{\circ} \mathrm{C}$, it is important to take into consideration the low phase angle determined at $0^{\circ} \mathrm{C}$ (relatively elastic response) and the high value obtained at the high temperature of $30^{\circ} \mathrm{C}$. 


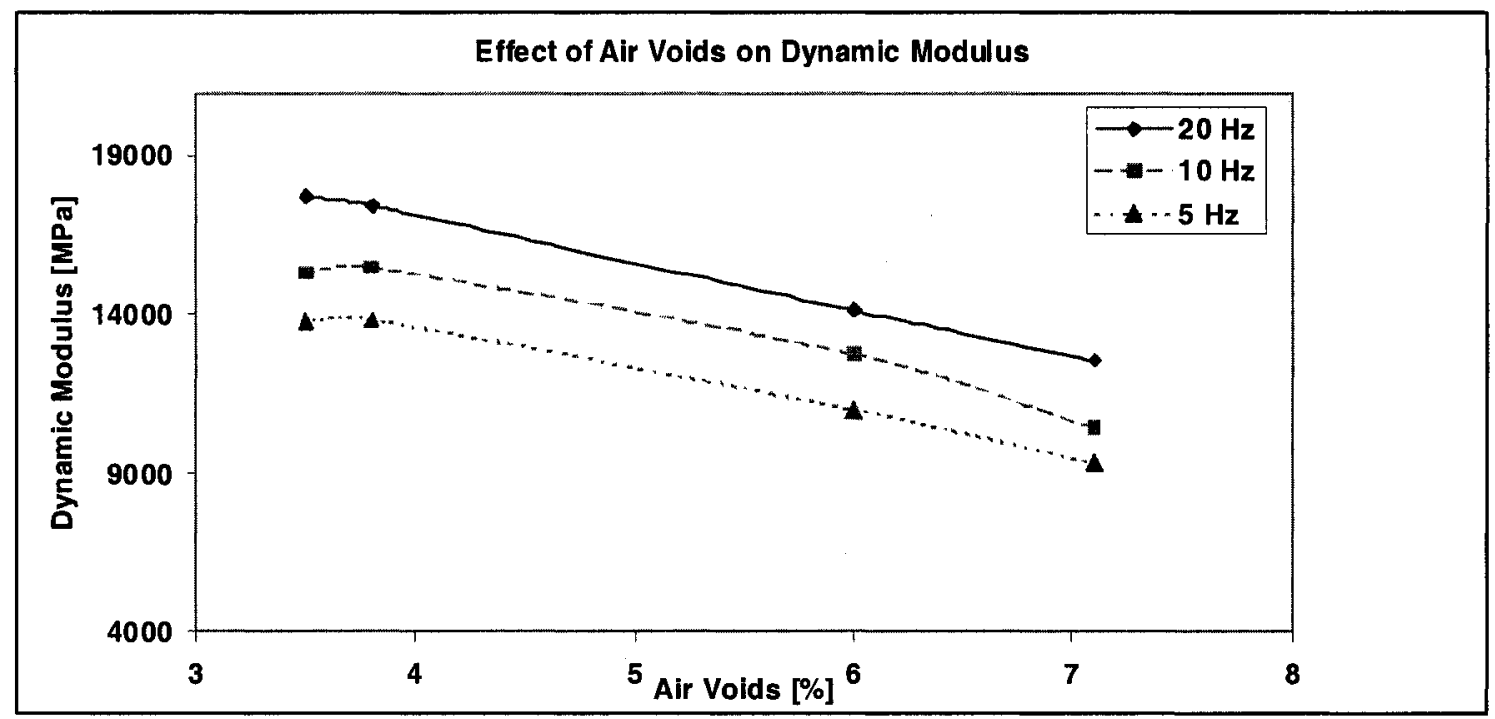

Figure 6.14. Dynamic modulus determined at $0^{\circ} \mathrm{C}$ at different air voids \%

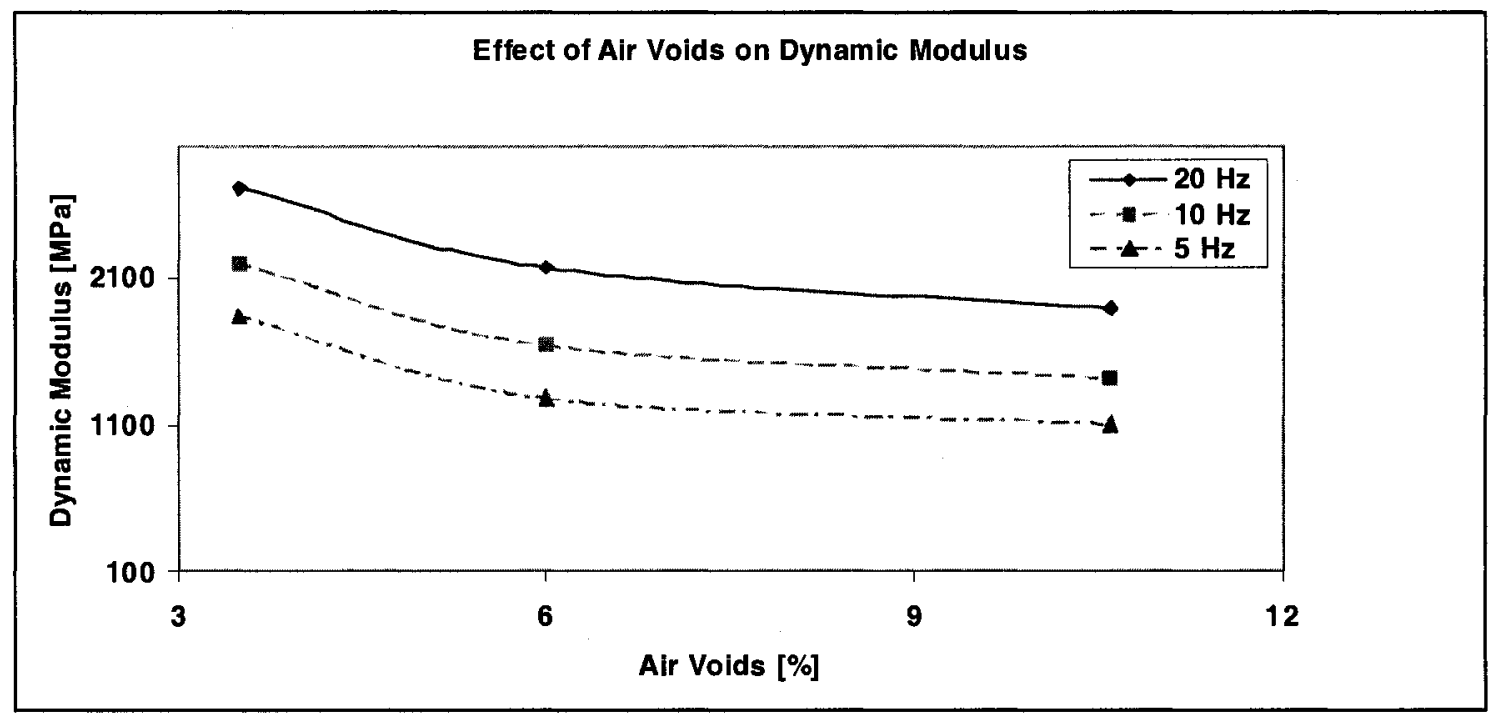

Figure 6.15. Dynamic modulus determined at $30^{\circ} \mathrm{C}$ at different air voids $\%$ 
Effect of Air Voids on Phase Angle

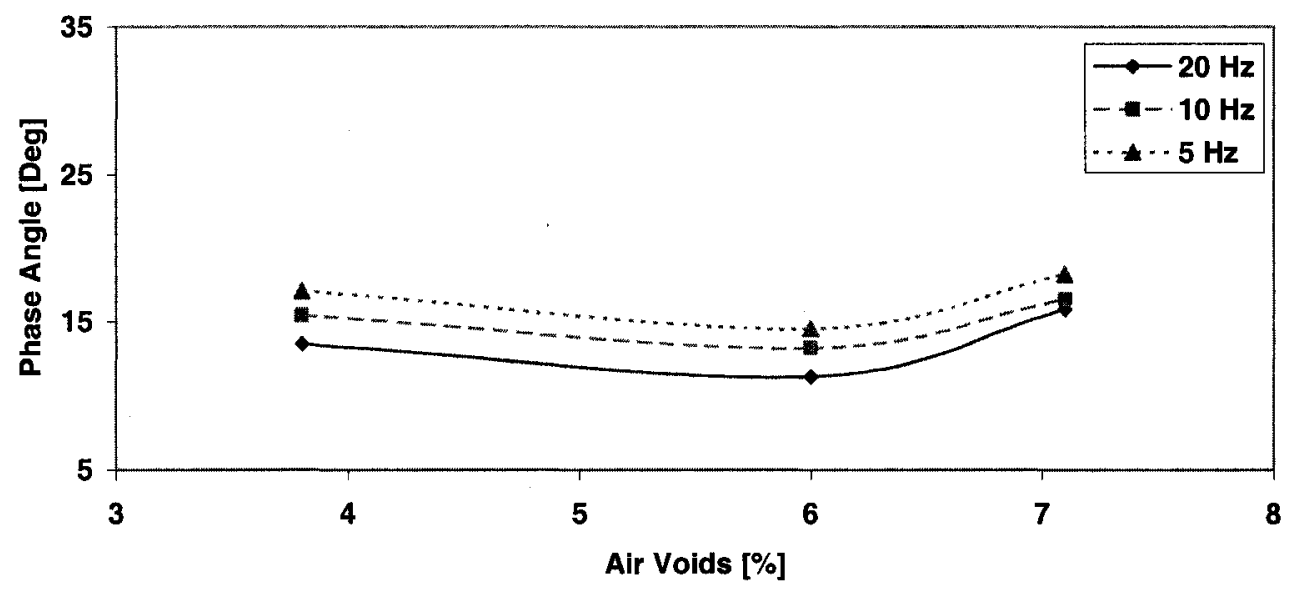

Figure 6.16. Phase angle determined at $0^{\circ} \mathrm{C}$ and different air voids $\%$

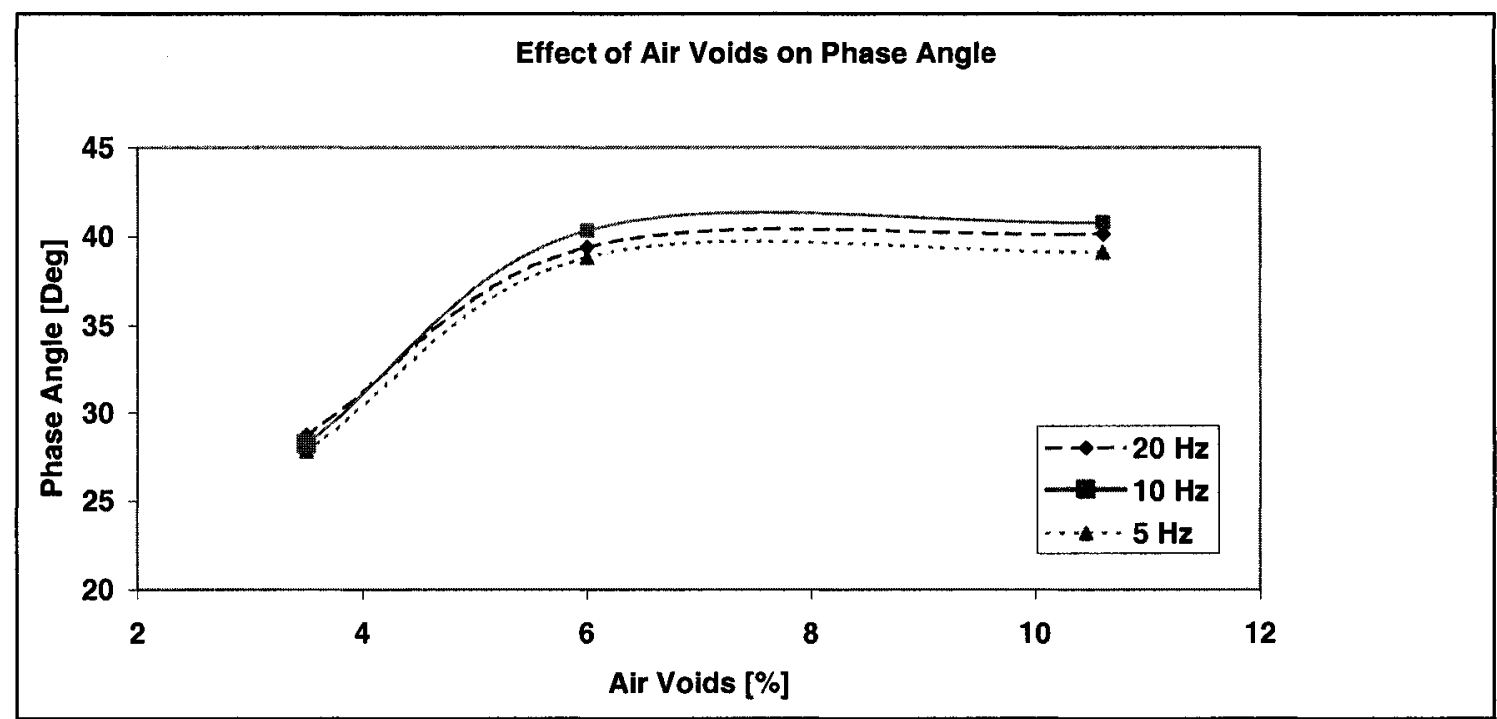

Figure 6.17. Phase angle determined at $30^{\circ} \mathrm{C}$ and different air voids \%

\subsubsection{Effect of Curing}

The designed experimental investigation discussed in Chapter 4 was intended to evaluate the effect of curing on the mechanistic properties of $\mathrm{AC}$ mixes based on the storage period between manufacturing the sample and testing it. A SuperPave mix (SP 2) prepared using a PG 52-34 binder was used to prepare samples with similar physical 
properties. These samples were tested at different ages. The determined dynamic moduli and phase angles are shown in Tables 6.17 and 6.18. The dynamic moduli and phase angles determined at the two test temperatures are plotted in Figures 6.18 to 6.21.

Table 6.17. Dynamic modulus (MPa) determined at -10 and $+40^{\circ} \mathrm{C}$ for samples with different ages

\begin{tabular}{|c|c|c|c|c|c|c|c|}
\hline \multirow{2}{*}{$\begin{array}{c}\text { Temperature } \\
{\left[{ }^{\circ} \mathrm{C}\right]}\end{array}$} & \multirow{2}{*}{$\begin{array}{l}\text { Age } \\
\text { [Day] }\end{array}$} & \multicolumn{6}{|c|}{ Frequency $[\mathrm{Hz}]$} \\
\hline & & 20 & 10 & 5 & 1 & 0.3 & 0.1 \\
\hline \multirow[t]{3}{*}{-10} & 1 & $2.27 \mathrm{E}+04$ & $2.18 \mathrm{E}+04$ & $2.16 \mathrm{E}+04$ & $2.12 \mathrm{E}+04$ & $1.56 \mathrm{E}+04$ & $1.14 \mathrm{E}+04$ \\
\hline & 6 & $2.69 E+04$ & $2.60 \mathrm{E}+04$ & $2.51 \mathrm{E}+04$ & $2.13 E+04$ & $1.88 \mathrm{E}+04$ & $1.62 \mathrm{E}+04$ \\
\hline & 30 & $3.30 \mathrm{E}+04$ & $3.15 \mathrm{E}+04$ & $3.05 \mathrm{E}+04$ & $2.64 \mathrm{E}+04$ & $2.34 \mathrm{E}+04$ & $2.03 E+04$ \\
\hline \multirow[t]{4}{*}{+40} & 1 & $3.58 \mathrm{E}+02$ & $2.53 E+02$ & $1.90 \mathrm{E}+02$ & $1.20 \mathrm{E}+02$ & $1.00 \mathrm{E}+02$ & $9.09 \mathrm{E}+01$ \\
\hline & 6 & $5.56 \mathrm{E}+02$ & $3.94 \mathrm{E}+02$ & $2.93 E+02$ & $1.60 \mathrm{E}+02$ & $1.10 \mathrm{E}+02$ & $8.00 \mathrm{E}+01$ \\
\hline & 16 & $6.56 \mathrm{E}+02$ & $5.00 \mathrm{E}+02$ & $3.89 \mathrm{E}+02$ & $2.32 \mathrm{E}+02$ & $1.70 \mathrm{E}+02$ & $1.40 \mathrm{E}+02$ \\
\hline & 30 & $7.90 \mathrm{E}+02$ & $6.04 \mathrm{E}+02$ & $4.80 \mathrm{E}+02$ & $3.10 \mathrm{E}+02$ & $2.50 \mathrm{E}+02$ & $2.12 \mathrm{E}+02$ \\
\hline
\end{tabular}

Table 6.18. Phase angles $\left({ }^{\circ}\right)$ determined at -10 and $+40^{\circ} \mathrm{C}$ for samples with different ages

\begin{tabular}{|c|c|c|c|c|c|c|c|}
\hline Temperature & Age & \multicolumn{7}{|c|}{ Frequency $[\mathrm{Hz}]$} \\
\cline { 3 - 8 }$\left[{ }^{\circ} \mathrm{C}\right]$ & {$[$ Day $]$} & 20 & 10 & 5 & 1 & 0.3 & 0.1 \\
\hline \multirow{4}{*}{-10} & 1 & 6.6 & 7.9 & 8.0 & 8.5 & 15.1 & 18.9 \\
\cline { 2 - 8 } & 6 & 5.4 & 6.1 & 6.8 & 10.4 & 13.6 & 16.3 \\
\hline & 30 & 6.0 & 6.0 & 6.5 & 9.8 & 12.9 & 14.8 \\
\hline \multirow{8}{*}{+40} & 1 & 50.3 & 47.7 & 35.0 & 26.5 & 20.0 & 15.0 \\
\hline & 6 & 42.8 & 30.8 & 32.5 & 36.2 & 37.4 & 33.2 \\
\cline { 2 - 8 } & 16 & 40.1 & 38.9 & 25.1 & 31.3 & 34.6 & 27.7 \\
\cline { 2 - 8 } & 30 & 45.2 & 41.6 & 23.1 & 25.5 & 28.1 & 21.8 \\
\hline
\end{tabular}

Both Figures 6.18 and 6.19 suggest that the AC material became stiffer with age, reflected in the increase in the dynamic modulus at both cold and warm test temperatures. The dynamic modulus determined at $-10^{\circ} \mathrm{C}$ for a sample stored for 30 days after manufacturing is $31 \%$ higher than the value determined by a test performed after 18 hours. The 18-hour period is close to the time at which a road will be opened to traffic after applying an overlay. Moreover, the same samples tested at $+40^{\circ} \mathrm{C}$ showed that the sample with a 30-day age was stiffer than the sample with 18-hour age where the 
dynamic modulus dropped by $58 \%$. Since cooling of the material for such a long time is not allowed in the field before opening the road to traffic (ranges from 1 to 24 hours), the trend detected in this investigation suggesting that aging that takes a relatively longer time must be considered in preparing road rehabilitation plans. This period, to be selected based on tests performed on a local road, should allow enough time for the chemical reaction between components of the asphalt cement and mineral aggregate to take place, and for the evaporation processes to take place for the mixture to achieve adequate stiffness before opening the road for traffic. The stiffness is expected to drop in the future because of damage accumulating during the service life of the road.

Figure 6.20 reflects the impact of curing on phase angle at relatively low test temperature $\left(0^{\circ} \mathrm{C}\right)$. The phase angle was small as expected at such a low temperature. The phase angle decreased between 18 hours and 6 days and then remained constant for the rest of the curing period at test frequencies.

However, at $+40^{\circ} \mathrm{C}$ the phase angles decreased slightly up to 6 days and then remained constant up to the last curing day, as shown in Figure 6.21. The above discussions suggest that $\mathrm{AC}$ mixtures continue to stiffen with age. However, the limited data obtained in this study about aging did not provide enough information to formulate a conclusion related to an ultimate stiffness where the chemical reactions cease to influence the stiffness. The process is more complicated because of the other factors that influence the stiffness of the mix, such as oxidative aging, which is outside the scope of this investigation. 


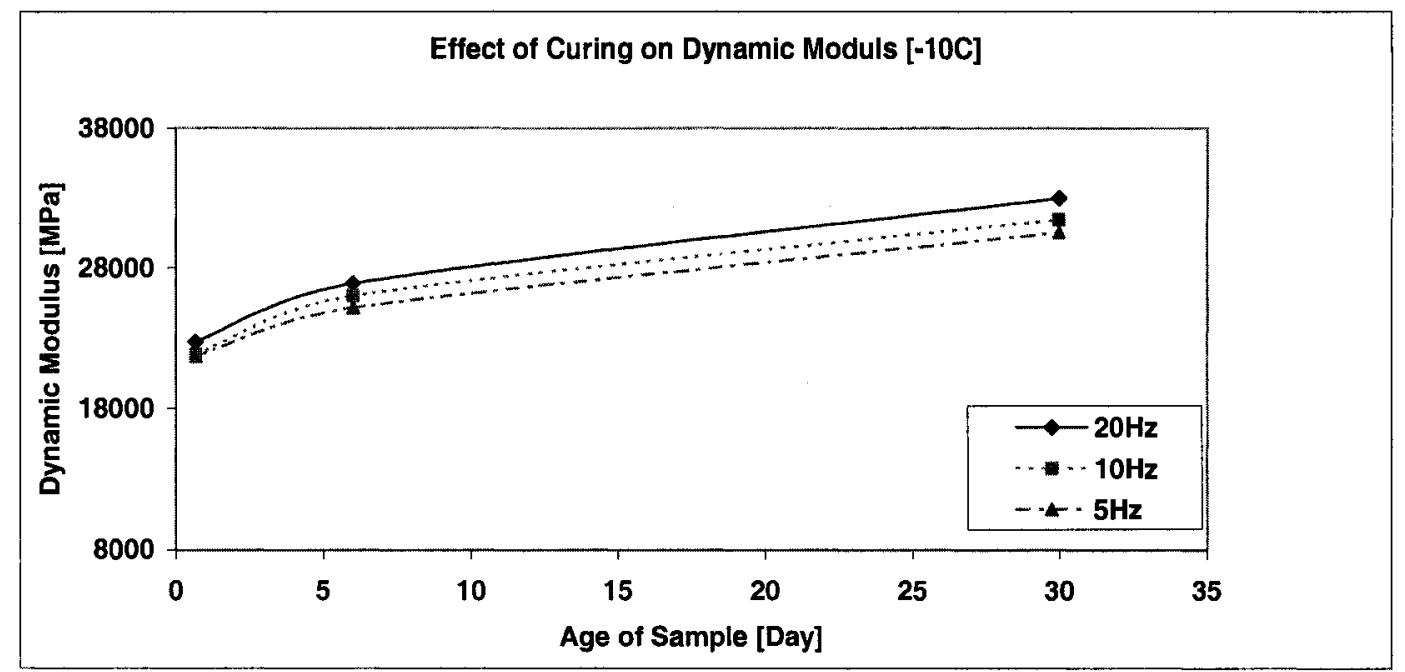

Figure 6.18. Dynamic modulus determined at $-10^{\circ} \mathrm{C}$ after different sample storage periods

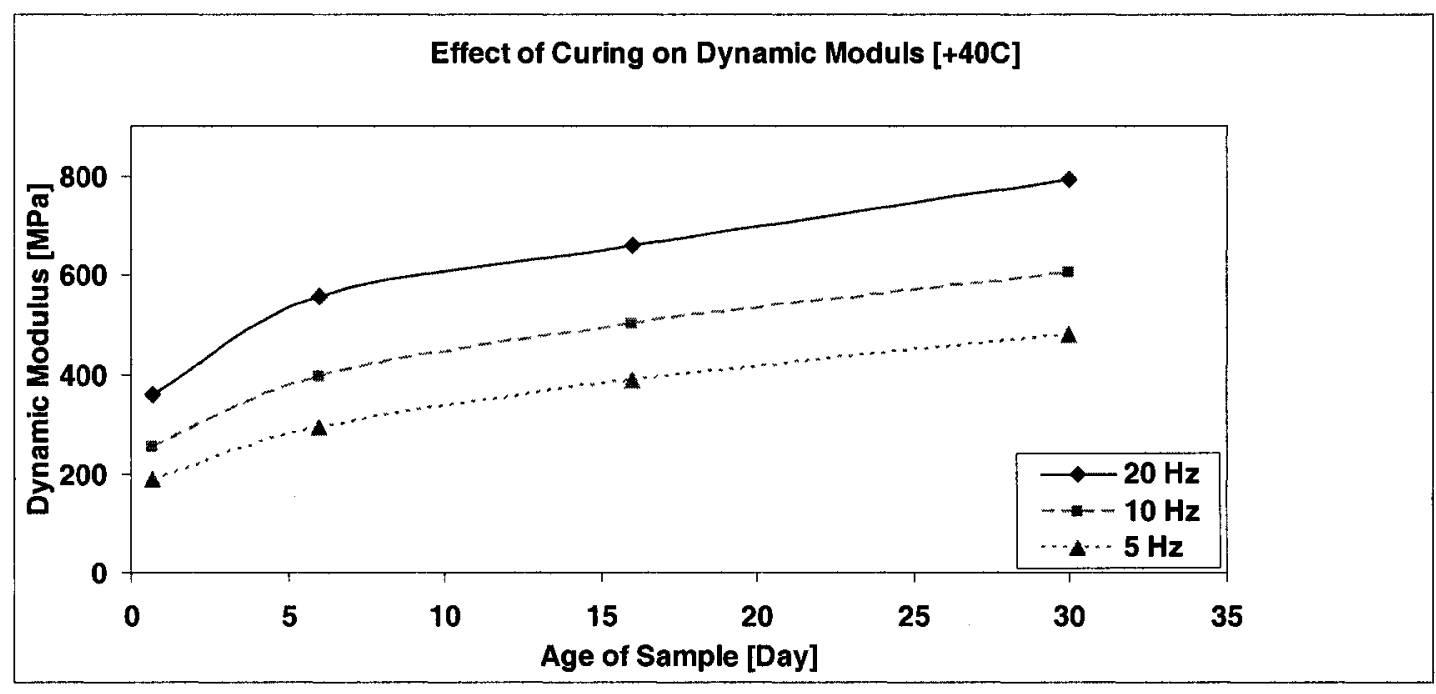

Figure 6.19. Dynamic modulus determined at $+40 \mathrm{oC}$ after different sample storage periods 


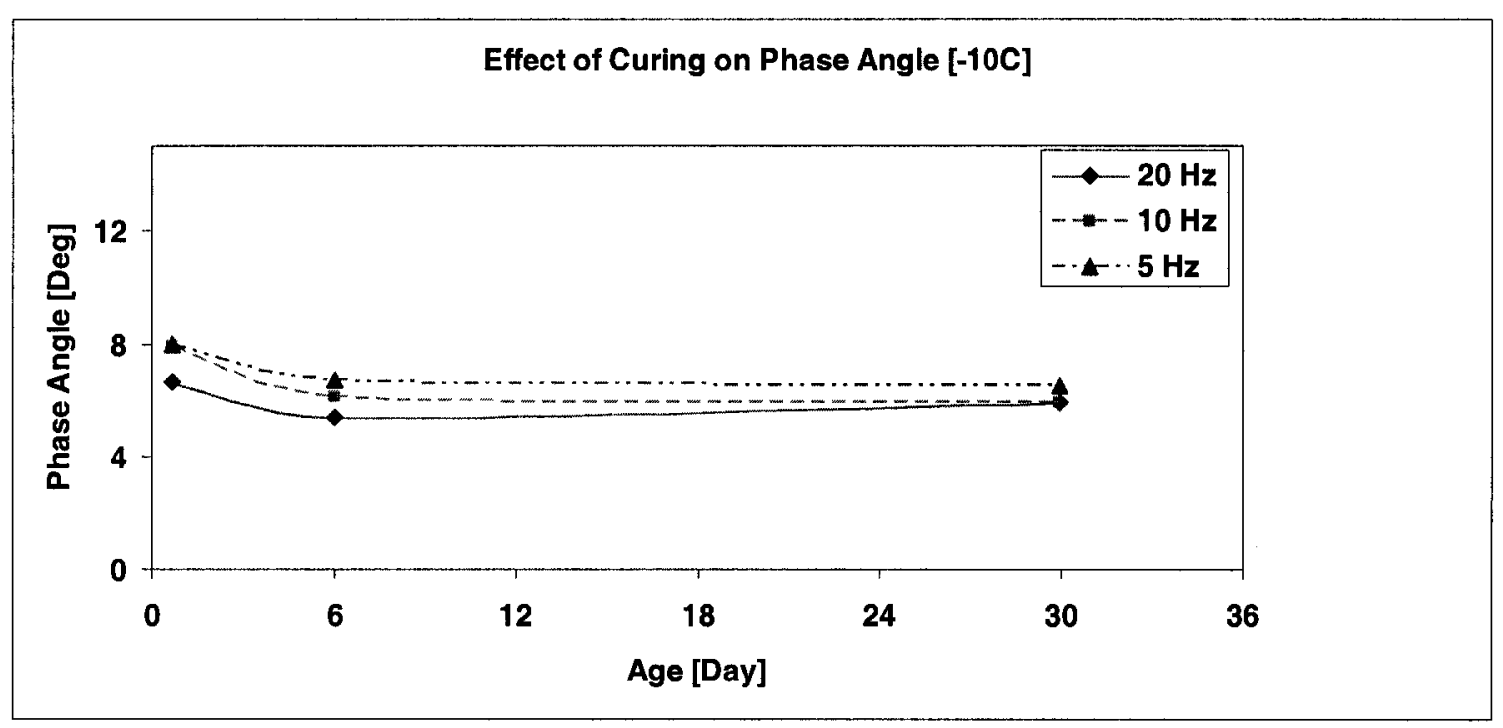

Figure 6.20. Phase angle determined at $-10^{\circ} \mathrm{C}$ after different sample storage periods

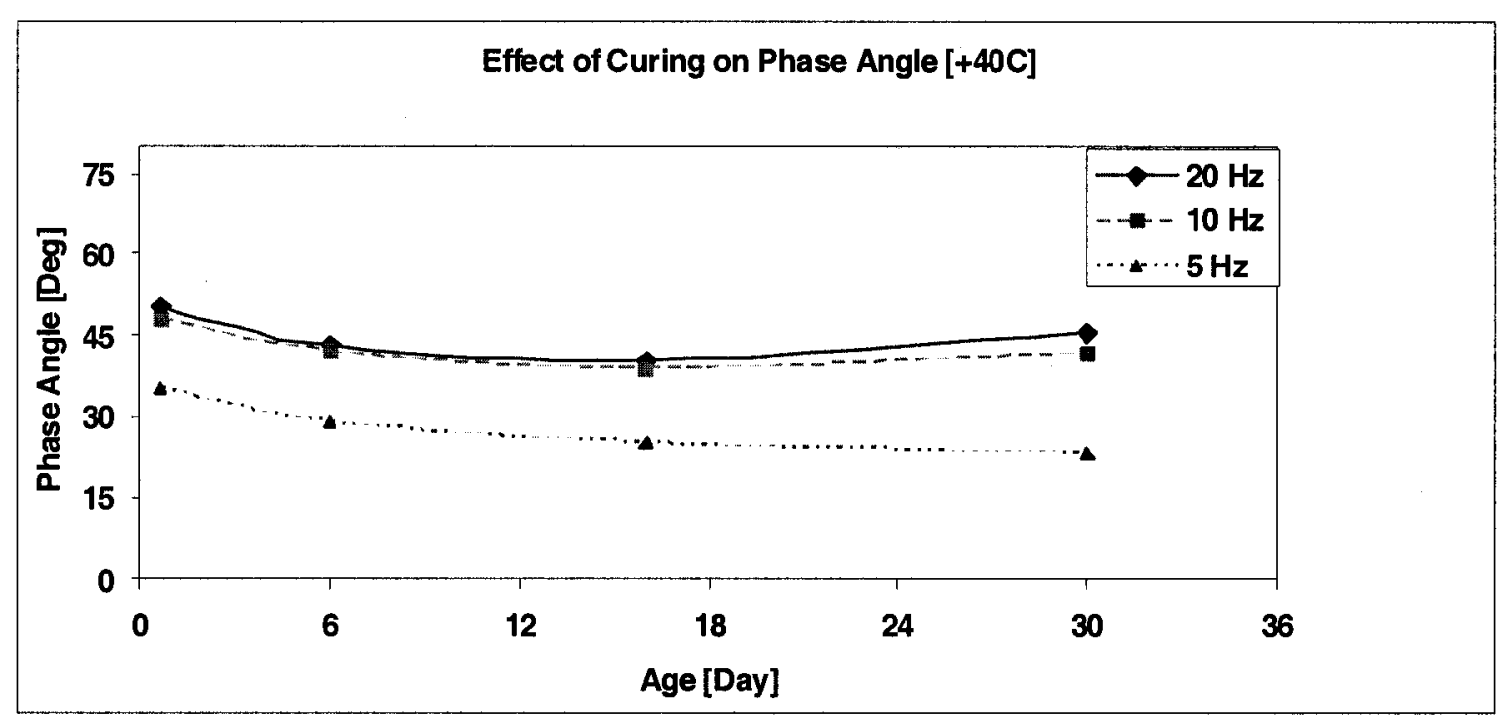

Figure 6.21. Phase angle determined at $+40 \mathrm{oC}$ after different sample storage periods

\subsubsection{Effect of Mix Design Procedure}

Although the SuperPave and Marshall mix designs seem to produce similar mixes, the comparison of their characteristics conducted in this study was intended to determine if there is a difference in the mechanistic response that may be associated with differences 
in the two volumetric design procedures. Two mixes, HMA 3 and SP 2, with the same maximum aggregate size, were prepared using the same binder (PG 52-34) and tested following the complex modulus test protocol. The results are shown in Tables 6.19 and 6.20 and are plotted in Figures 6.22 to 6.25 .

Table 6.19. Dynamic modulus for SP 2 and HMA 3 determined at different temperatures using 20 and $0.3 \mathrm{~Hz}$

\begin{tabular}{|c|c|c|c|c|}
\hline \multirow[b]{2}{*}{ Temperature $\left[{ }^{\circ} \mathrm{C}\right]$} & \multicolumn{2}{|c|}{$\begin{array}{l}\text { Dynamic Modulus [MPa] } \\
20 \mathrm{~Hz}\end{array}$} & \multicolumn{2}{|c|}{$\begin{array}{c}\text { Dynamic Modulus [MPa] } \\
0.3 \mathrm{~Hz}\end{array}$} \\
\hline & $\begin{array}{c}\text { HMA 3 } \\
\text { (Marshall) }\end{array}$ & $\begin{array}{c}\text { SP 2 } \\
\text { (SuperPave) }\end{array}$ & $\begin{array}{c}\text { HMA 3 } \\
\text { (Marshall) }\end{array}$ & $\begin{array}{c}\text { SP 2 } \\
\text { (SuperPave) }\end{array}$ \\
\hline 0 & 10773.1 & 12979.8 & 4583.3 & 4380 \\
\hline 20 & 3042.0 & 2840 & 700.0 & 570 \\
\hline 30 & 1093.2 & 960 & 225.0 & 170 \\
\hline 40 & 551.7 & 3578947 & 1333 & 100 \\
\hline
\end{tabular}

Table 6.20. Phase angle for SP2 and HMA 3 determined at different temperatures using 20 and $0.3 \mathrm{~Hz}$

\begin{tabular}{|c|c|c|c|c|}
\hline \multirow[b]{2}{*}{ Temperature $\left[{ }^{\circ} \mathrm{C}\right.$} & \multicolumn{2}{|c|}{ Phase Angle $\left[{ }^{\circ}\right], 20 \mathrm{~Hz}$} & \multicolumn{2}{|c|}{ Phase Angle $\left[{ }^{\circ}\right], 0.3 \mathrm{~Hz}$} \\
\hline & $\begin{array}{c}\text { HMA 3 } \\
\text { (Marshall) }\end{array}$ & $\begin{array}{c}\text { SP 2 } \\
\text { (SuperPave) }\end{array}$ & $\begin{array}{l}\text { HMA } 3 \\
\text { (Marshall) }\end{array}$ & $\begin{array}{c}\text { SP 2 } \\
\text { (SuperPave) }\end{array}$ \\
\hline-10 & 8 & 7 & 16 & 15 \\
\hline 0 & 16 & 17 & 26 & 28 \\
\hline 20 & 35 & 40 & 40 & 40 \\
\hline 30 & $\overline{43}$ & 51 & 42 & 39 \\
\hline 40 & 44 & 50 & 35 & 20 \\
\hline
\end{tabular}

Figure 6.22 shows the dynamic modulus-temperature relationship for SP 2 and HMA 3 evaluated at a loading frequency of $0.3 \mathrm{~Hz}$, where the mixes prepared according to the two mix design procedure showed quite similar values. Although similar binders were used in the samples, the test performed at $20 \mathrm{~Hz}$ showed $15 \%$ higher modulus for the SP 2 mix compared with the HMA $3 \mathrm{mix}$ as shown in Figure 6.23. There is no clear explanation for the recorded deviation from the result obtained at $0.3 \mathrm{~Hz}$. There was a 
slight difference between the gradations of the two mixes, which was not expected to be reflected in the low-temperature response.

The difference in phase angles determined for HMA 3 and SP 2 at a high loading frequency of $20 \mathrm{~Hz}$ is negligible at all temperatures. However, the phase angle determined at $0.3 \mathrm{~Hz}$ showed a difference between the two mixes at the high temperature of $+40^{\circ} \mathrm{C}$. The phase angle determined for the HMA 3 was $42 \%$ higher than for the SP 2 mix. The HMA 3 included $40 \%$ natural sand while the SP 2 had no sand. During the SHRP project, high sand content was identified as a factor that may promote mix tenderness. Measures to limit its use have been incorporated in SuperPave mix design specifications (a restricted zone incorporated in the gradation curve). According to physical properties shown earlier in Table 4.10, the HMA 3 mix included more sand than the SP 2 mix, which may explain the tenderness of the HMA 3 mix as reflected in low dynamic modulus values. Thus, based on the results of the complex modulus test, the SP 2 proved to be more stable than HMA 3 at higher temperatures.

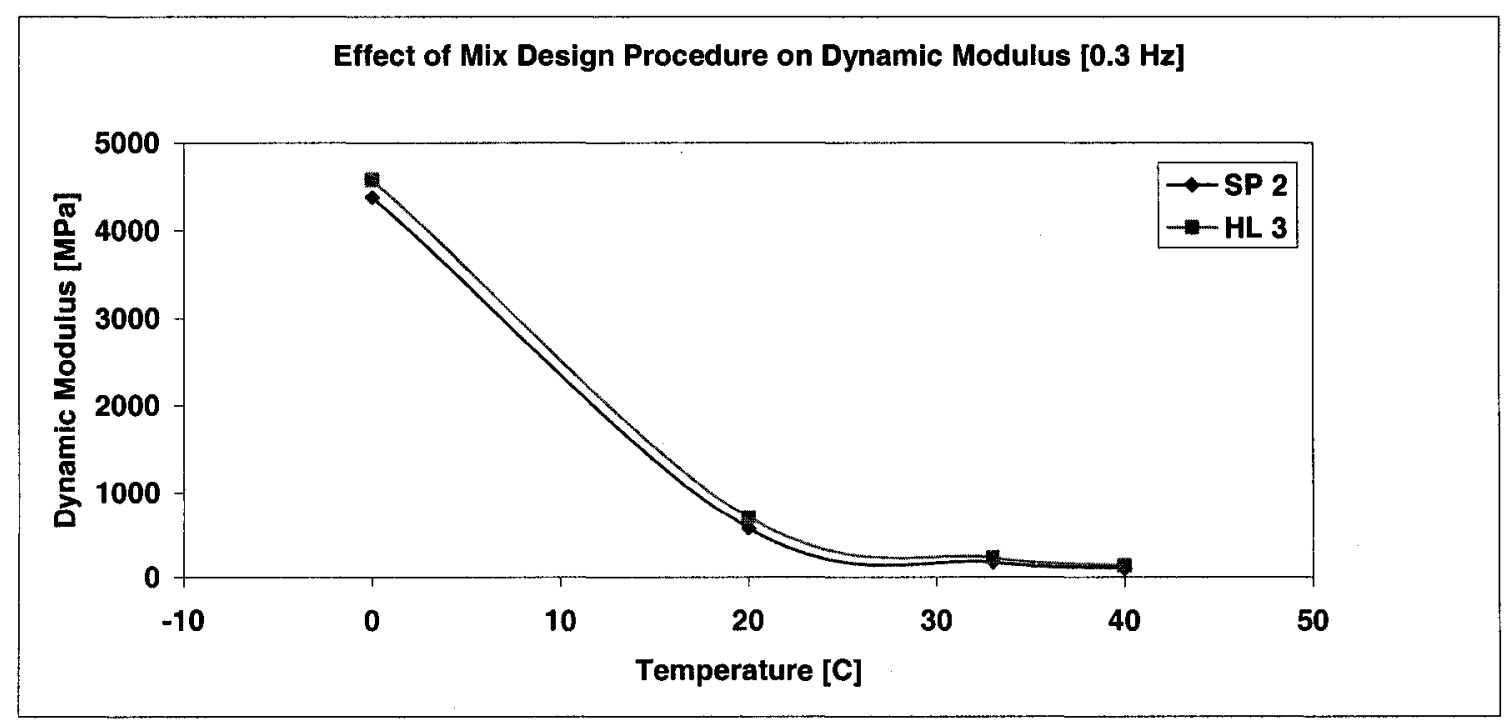

Figure 6.22. Effect of mix design procedure on dynamic modulus [0.3 Hz] 


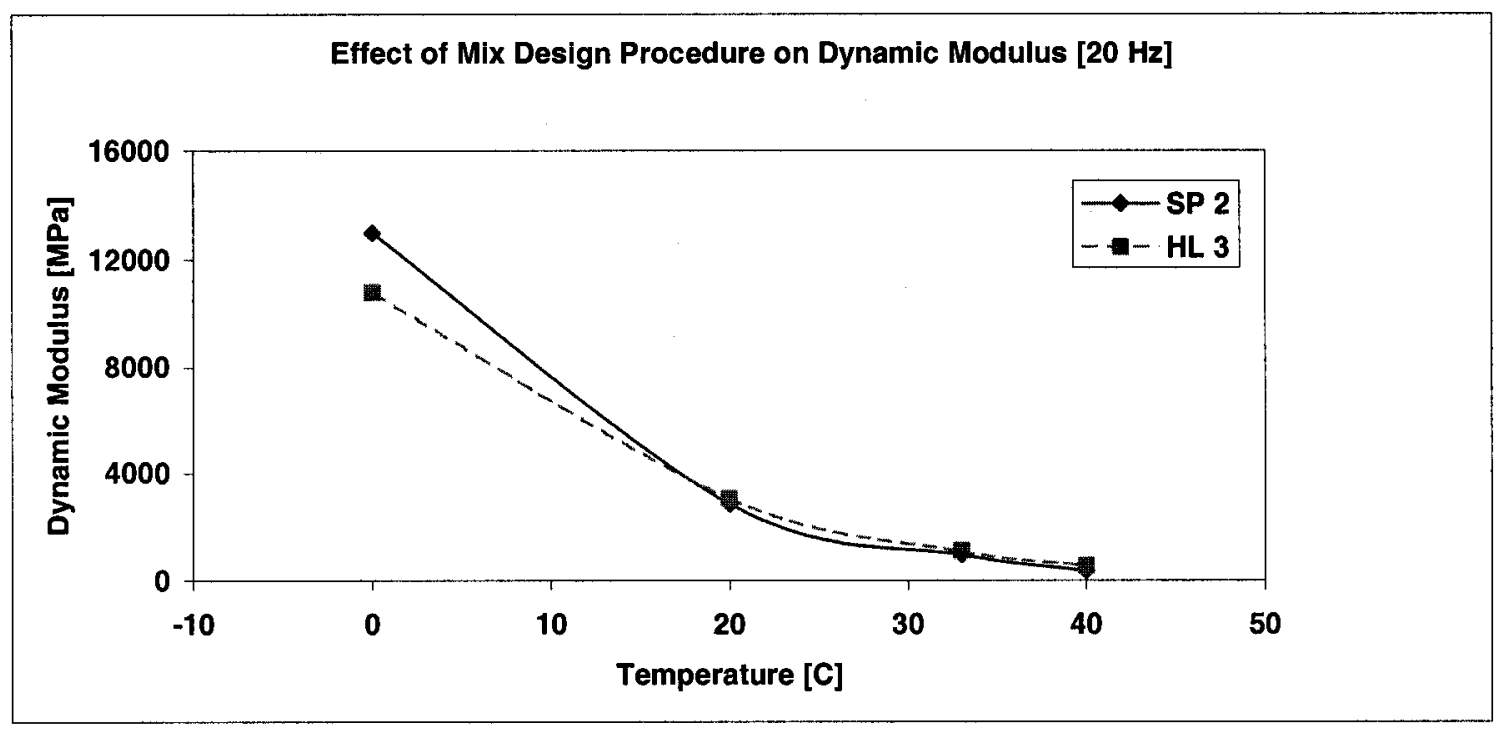

Figure 6.23. Effect of mix design procedure on dynamic modulus [20 Hz]

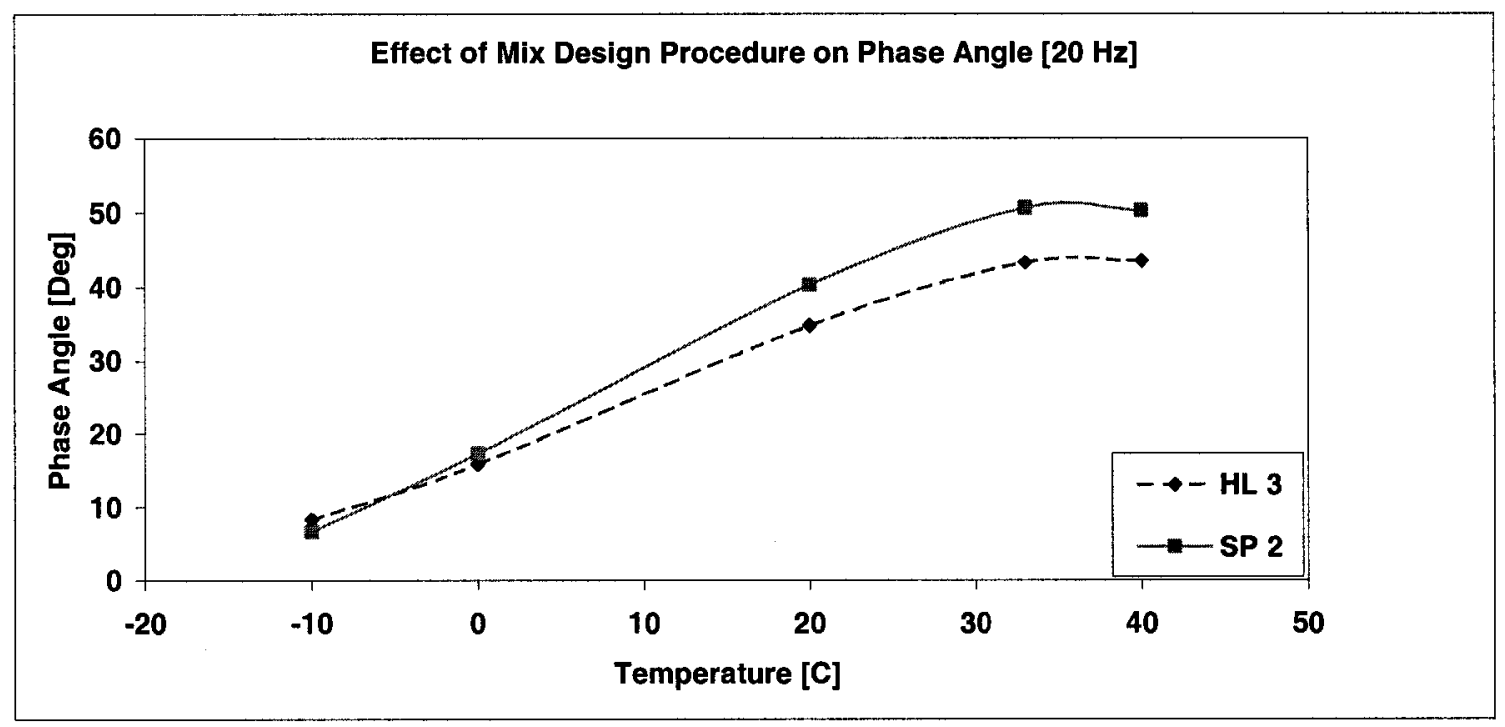

Figure 6.24. Effect of mix design procedure on phase angle $[20 \mathrm{~Hz}]$ 


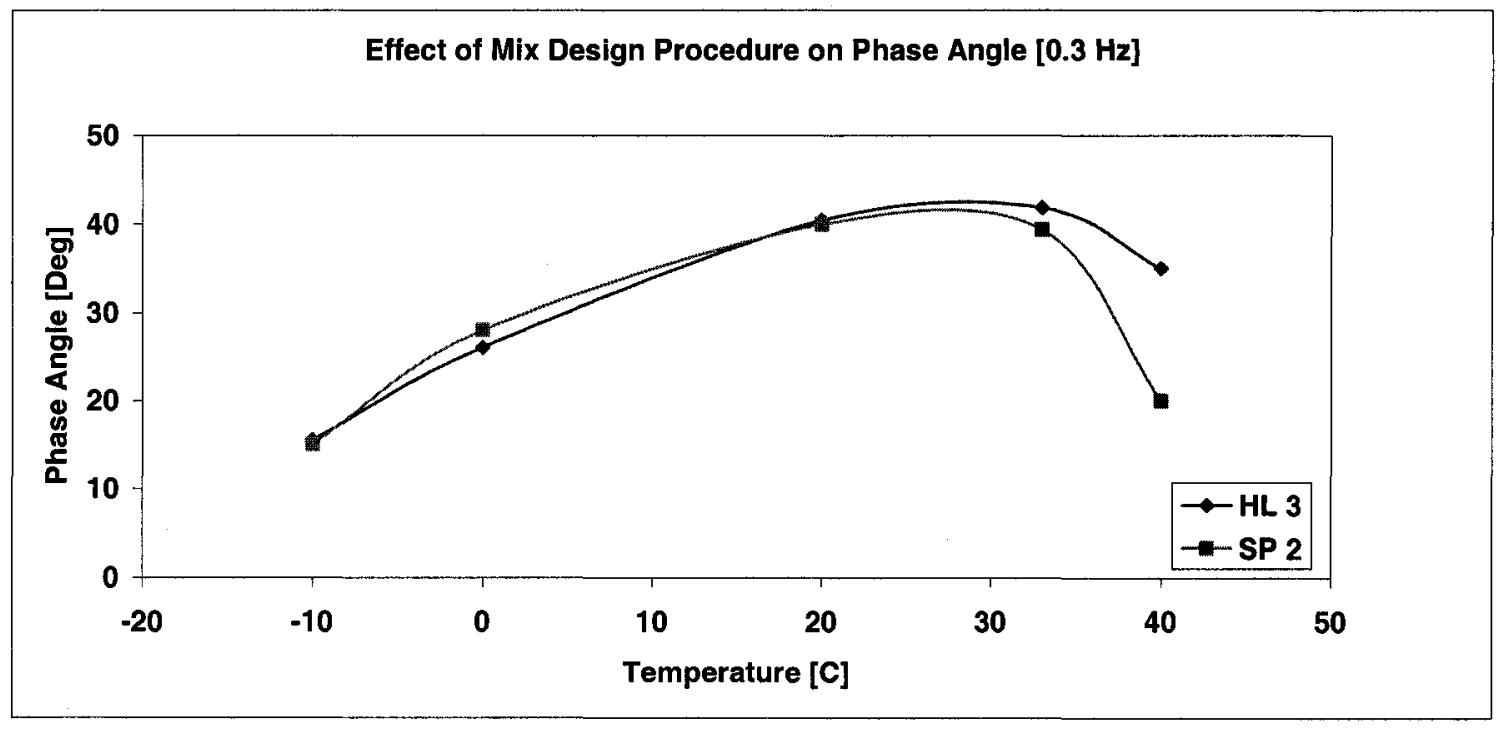

Figure 6.25. Effect of mix design procedure on phase angle [0.3 Hz] 


\section{CHAPTER 7 \\ IMPLEMENTATION OF THE COMPLEX MODULUS PARAMETERS}

\subsection{Introduction}

The complex modulus approach has been selected in this thesis for characterizing asphalt concrete because of its effectiveness in capturing the mechanistic response of the material. The test procedure developed in the thesis proved to be sensitive to the major elements that influence the performance of asphalt concrete materials; namely traffic and environmental conditions. It is no surprise that the complex modulus is being considered in the proposed ASSHTO 2002 Design Guide and in other applications such the VEROAD program developed in the Netherlands.

Given the popularity of the complex modulus concept and its probable adoption by many organizations, the data generated in this thesis were used to develop a pilot database to act as a Material Library for users of analytical models and other applications such as rating of mixes or the development of construction quality control systems. The information that can serve as input in a variety of applications was tabulated in the Material Library attached to this thesis (Appendix B). The developed library encompasses the data in the format needed for application in the 2002 Design Guide and other applications that make use of the Huet-Sayegh rheological model. The Huet-Sayegh model is more popular in Europe and was used extensively and successfully in the VEROAD program. Other possible application relates to using results of the laboratory tests, in terms of the dynamic moduli and phase angles, in developing new rheological models similar to the predictive equations incorporated in Level 3 of the ASSHTO 2002 Design Guide. The following subsections describe the Material Library and the 
discussions related to the above three (typical) applications of the proposed characterization technique.

\subsection{Material Library}

The database, entitled the "Material Library," was designed taking into consideration numerous possible applications such as those described earlier. These applications require the type of physical and mechanical properties produced in the laboratory investigation performed as part of this study. Some applications will pick specific material properties directly from the Library and use them as input for the analytical models that will analyze pavements to produce design solutions. Other applications, intended as simple design schemes, use only physical properties produced by the user from simple laboratory tests to arrive at the properties required by mechanistic models. This type of application, depicted in Figure 7.1, enables the user to identify a material with similar physical properties in the Library and proceed to pick the corresponding mechanical properties from the library so that the mechanistic design process that requires such properties can be used. Although not as precise as model runs performed with the direct results of mechanical tests, this approach enables users that do not require any expertise or equipment to perform the complex modulus test technique developed in this thesis to benefit from the application of mechanistic models. The other indirect way to avoid the routine performance of mechanical tests involves the establishment of predictive equations that link the physical properties to the corresponding mechanical ones similar to those established for the ASSHTO 2002 Guide and evaluated in Subsection 7.5. 
The large number of parameters needed to perform the above applications and the manner in which these applications are performed (software), necessitate establishing an electronic database as an ideal format for the library. However, for illustration purposes, a hard copy of the Material Library was constructed as shown in Appendix B. Two typical line entries are shown in Table 7.1. Abbreviations and notations used in Table 7.1 are described in the "List of Symbols and Definitions"

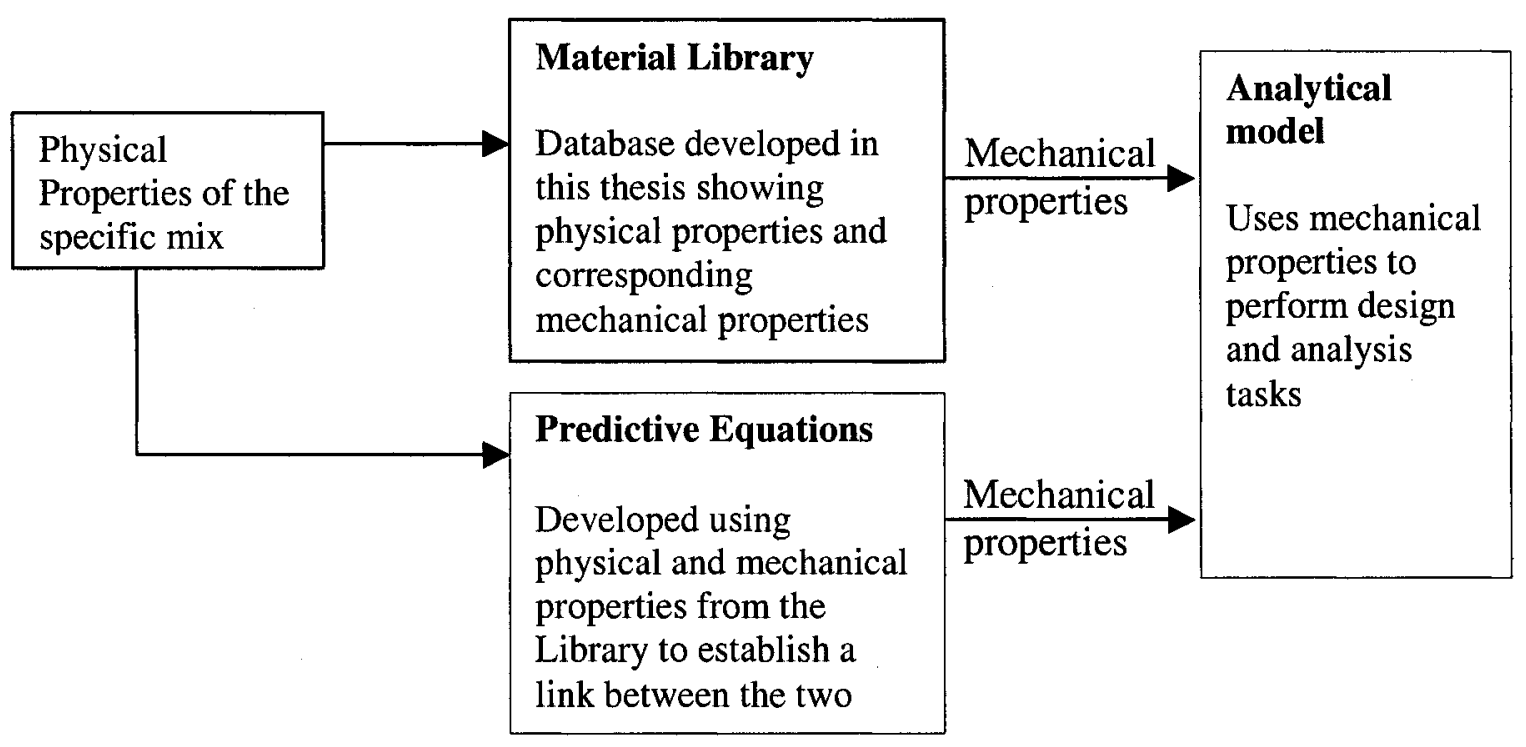

Figure 7.1. Library application in simplified design practices

Components of the Material Library were constructed using practices and terms known to the industry. The first section of the Library includes mix designations including the code established in this thesis and others that may be assigned by users conforming to local names of the mix. The mix should be identified by the physical properties listed in the Library. The other main components are:

- Binder characteristics, including the physical properties and some mechanical properties (usually provided by the manufacturer based on specifications developed by the SHRP project). 
- Aggregate characteristics, including specific gravity and gradation according to specific sieve sizes. These are all properties that may be produced using standard ASSHTO tests procedures commonly performed by the industry.

- Mix characteristics, which consist of three components:

1. Standard physical properties including the density achieved by compaction (the percentage of air voids in the mix is the most commonly used property), binder content. These are properties that are usually calculated to reflect the asphalt cement present in voids and as a film around aggregate particles.

2. The dynamic modulus determined using the complex modulus testing technique and the data-processing procedure established in this thesis. The dynamic modulus values are listed according to the loading frequency and temperature conditions used at each test stage (specific ranges are specified in the ASSHTO 2002 Guide, which requires this category of data).

3. The eight parameters required by the Huet-Sayegh rheological model. This model was used in many analytical models to capture the contribution of the AC layer to the overall pavement response. The National Research Council Canada, where this experimental investigation was performed, is developing such a model based on the results of this investigation. 
Table 7.1. Material library containing typical data pertaining to HMA 1 and SP 2 mixes

\begin{tabular}{|c|c|c|c|c|c|c|c|c|c|c|c|c|c|c|c|c|c|c|}
\hline \multicolumn{2}{|c|}{ Mix ID } & \multicolumn{9}{|c|}{ Binder Characteristics } & \multicolumn{8}{|c|}{ Aggregates Characteristics } \\
\hline Designation & Local Name & PG Type & Gb & S. Point & A. Viscosity & K. Viscosity & Pen. & R. Viscosity & $\mathrm{G}^{*}$ & $\operatorname{Sin} \delta$ & Dmax & Dnom & $\mathrm{R} 3 / 4$ & $\mathrm{R} 3 / 8$ & R\#4 & P200 & Gse & Gsb \\
\hline HMA 1 & HL 8 & $58-22$ & 1.025 & 125 & 2200 & 340 & 90 & 0.323 & $\mathrm{n} / \mathrm{a}$ & $\mathrm{n} / \mathrm{a}$ & 19 & 16 & 3.8 & 46.2 & 51.4 & 0.5 & 2.728 & 2.74 \\
\hline SP 2 & $\mathrm{n} / \mathrm{a}$ & $64-34$ & 1.028 & $\mathbf{n} / \mathbf{a}$ & n/a & 340 & 85 & 1.05 & $\mathbf{n} / \mathbf{a}$ & $\mathrm{n} / \mathrm{a}$ & 16 & 12.5 & 5 & 24.3 & 32.1 & 0.9 & 2.789 & 2.755 \\
\hline
\end{tabular}

Table 7.1: Continued

\begin{tabular}{|c|c|c|c|c|c|c|c|c|c|c|c|c|c|c|c|c|c|c|c|c|c|c|c|c|c|}
\hline \multicolumn{26}{|c|}{ AC Mix } \\
\hline \multicolumn{9}{|c|}{ Physical properties } & \multicolumn{8}{|c|}{$\begin{array}{l}\text { Mechanical Properties for AASHTO } 2002 \text { Design Guide } \\
\text { Applications }\end{array}$} & \multicolumn{9}{|c|}{ Mechanical Properties for Huet-Sayegh Model Applications } \\
\hline \multirow[t]{5}{*}{ Mix ID } & \multirow[t]{5}{*}{$\mathrm{AC} \%$} & \multirow[t]{5}{*}{ Agg \% } & MSG & BSG & $\mathrm{Av} \%$ & VMA\% & VFA\% 1 & Pbeff\% & \multicolumn{2}{|c|}{ T (C) } & \multirow{2}{*}{\begin{tabular}{|c|}
20 \\
13347
\end{tabular}} & \multirow{2}{*}{\begin{tabular}{|c|}
10 \\
12921
\end{tabular}} & \multirow{2}{*}{\begin{tabular}{|c|}
5 \\
11929
\end{tabular}} & \multirow{2}{*}{$\frac{1}{10991}$} & \multirow{2}{*}{\begin{tabular}{|c|}
0.3 \\
10322 \\
\end{tabular}} & \multirow{2}{*}{$\frac{0.1}{9602}$} & \multirow[b]{3}{*}{ Parameter } & \multirow[b]{3}{*}{$\mathrm{E}_{\infty}$} & \multirow[b]{3}{*}{$E_{o}$} & \multirow[b]{3}{*}{$\delta$} & & & & & \\
\hline & & & & & & & & & & $\mathbf{E}^{*}$ & & & & & & & & & & & & & & & \\
\hline & & & & & & & & & -10 & $\phi$ & 3.3 & 3.5 & 4.3 & 5.8 & 6.6 & 9.5 & & & & & k & $\mathbf{h}$ & $\mathbf{a}$ & b & $\mathrm{c}$ \\
\hline & & & & & & & & & & $\mid \mathbf{E}^{*}$ & 10033 & 9539 & 8833 & 7939 & 7042 & 6275 & & & & & & & & & \\
\hline & & & & & & & & & 0 & $\phi$ & 7.6 & 7.7 & 8.7 & 10.1 & 13.1 & 16.2 & Value & 16440 & 60 & 1.480 & 0.195 & 0.600 & 0.868 & -0.285 & 0.001 \\
\hline & & & & & & & & & & E* & 7849 & 6966 & 6222 & 4815 & 3842 & 3068 & & & & & & & & & \\
\hline HMA 1 & 4.5 & 95.5 & 2.540 & 2.412 & 5.04 & 15.9 & 68.4 & 10.9 & 20 & $\phi$ & 12.3 & 13.5 & 15.6 & 20.1 & 23.8 & 27.5 & & & & & & & & & \\
\hline & & & & & & & & & & $\mathrm{E}^{*}$ & 2483 & 1891 & 1458 & 817 & 533 & 375 & & & & & & & & & \\
\hline & & & & & & & & & 30 & $\phi$ & 34.0 & 36.8 & 38.3 & 40.4 & 40.8 & 37.6 & & & & & & & & & \\
\hline & & & & & & & & & & $\mid \mathrm{E}^{*}$ & 825 & 608 & 467 & 269 & 200 & 167 & & & & & & & & & \\
\hline & & & & & & & & & 40 & $\phi$ & 43.0 & 42.6 & 40.7 & 36.4 & 31.4 & 26.5 & & & & & & & & & \\
\hline & & & & & & & & & $T(C)$ & $(\mathrm{Hz})$ & 20 & 10 & \begin{tabular}{|l|}
5 \\
\end{tabular} & 1 & 0.3 & 0.1 & & & & & & & & & \\
\hline & & & & & & & & & & $\mid \mathrm{E}^{*}$ & 19726 & 18385 & 16904 & 14535 & 12890 & 11460 & & & & & & & & & \\
\hline & & & & & & & & & -10 & $\phi$ & \begin{tabular}{|l|}
6.4 \\
\end{tabular} & 7.8 & \begin{tabular}{|l|}
8.8 \\
\end{tabular} & 10.6 & 12.9 & 14.3 & Parameter & $\mathrm{E}_{\infty}$ & $\mathrm{E}_{\mathrm{o}}$ & $\delta$ & $\mathrm{k}$ & $\mathrm{h}$ & a & b & $\mathrm{c}$ \\
\hline & & & & & & & & & & $\mathrm{E}^{*} \mathrm{C}$ & 14121 & 12737 & 9293 & 7610 & 6300 & 4760 & & & & & & & & & \\
\hline & & & & & & & & & 0 & $\phi$ & 11.3 & 13.2 & 14.5 & 17.4 & 20.0 & 21.9 & Value & 28373 & 127 & 2.318 & 0.229 & 0.531 & $|-0.307|$ & -0.265 & 0.001 \\
\hline & & & & & & & & & & $\mathbf{E}^{*}$ & 4700 & 4140 & 3330 & 2218 & 1660 & 1300 & & & & & & & & & \\
\hline SP 2 & 4.5 & 95.5 & 2.590 & 2.435 & 6.0 & 15.6 & 61.6 & 9.6 & 20 & $\phi$ & 28.5 & 31.2 & 32.3 & 32.6 & 32.2 & 31.0 & & & & & & & & & \\
\hline & & & & & & & & & & $\left|\mathrm{E}^{*}\right|$ & 2168 & 1644 & 1284 & 800 & 580 & 460 & & & & & & & & & \\
\hline & & & & & & & & & 30 & $\phi$ & 39.4 & 40.3 & 38.8 & 35.9 & 33.7 & 31.1 & & & & & & & & & \\
\hline & & & & & & & & & & $\mathrm{E}^{*} \mid$ & 910 & 717 & 570 & 390 & 300 & 250 & & & & & & & & & \\
\hline & & & & & & & & & 40 & $\phi$ & 39.2 & 37.9 & 27.6 & 27.5 & 31.5 & 25.7 & & & & & & & & & \\
\hline
\end{tabular}


Results and analysis reflecting the manner in which the applications make use of the complex modulus data are discussed in the following subsections.

\subsection{The AASHTO 2002 Design Guide Application}

The complex modulus characterization technique was incorporated in all three proposed design levels of the AASHTO 2002 Design Guide. More precisely, the primary stiffness property of interest for asphalt concrete materials is the dynamic modulus, which is known to be time-temperature dependent. In order to account for temperature and rate of loading, the 2002 Guide requires that the dynamic modulus of asphalt concrete materials be determined from a master curve that is constructed at a reference temperature of 21.1 ${ }^{\circ} \mathrm{C}\left(70^{\circ} \mathrm{F}\right)$ as described in Chapter 3.

For level 1 design, the guide requires conducting a stress-controlled laboratory complex modulus test at different temperatures and loading frequencies for the mix of interest. Such information can be produced using the complex modulus procedure described in Chapter 4 and the data reduction procedure discussed earlier in Chapter 5. The required properties are similar to those produced in this thesis such as the typical example given in Table 7.2 for the HMA 1 mix prepared using a PG 58-22 binder. These results were used to plot the stiffness-frequency relationship produced at different temperatures as shown in Figure 7.2 as proposed in the ASSHTO 2002 Design Guide.

Table 7.2. Dynamic moduli (MPa) for the HMA 1 mix (using PG 58-22 binder)

\begin{tabular}{|c|c|c|c|c|c|c|}
\hline \multirow{2}{*}{$\begin{array}{c}\text { Temperature } \\
{\left[{ }^{\circ} \mathrm{C}\right]}\end{array}$} & \multicolumn{6}{|c|}{ Frequency $(\mathrm{Hz})$} \\
\hline & 20 & 10 & 5 & 1 & 0.3 & 0.1 \\
\hline-10 & 11847 & 11791 & 11704 & 10615 & 9798 & 8908 \\
\hline 0 & 11190 & 10125 & 9273 & 7686 & 6467 & 5458 \\
\hline 20 & 8517 & 7200 & 6258 & 4450 & 3325 & 2442 \\
\hline 30 & 2686 & 1958 & 1492 & 783 & 475 & 308 \\
\hline 40 & 1192 & 832 & 600 & 292 & 175 & 117 \\
\hline
\end{tabular}


The AASHTO 2002 Design Guide uses this relationship to construct a master curve by fitting a nonlinear sigmoidal function using Equation 3.16. The master curve constructed using the test data of the HMA 3 mix shown earlier in Table 7.2 is presented in Figure 7.3 .

Master curves constructed for two distinct mixes (HMA 3 and HMA 1) prepared with the same Performance-Grade binder (PG 58-22) are shown in Figure 7.4. In the low modulus state, usually at elevated test temperatures and low loading frequencies, the responses of the two mixes are quite close to each other. However, the two mixes reflected uniquely different characteristic responses at the medium and high modulus states. The dynamic modulus values for the dense graded HMA 3 mix with the usually high binder content are much higher than that of the HMA 1 mix. Although the aggregate fraction in the mix is known to influence the mechanistic response, this observed difference may be attributed mainly to the high binder content in the HMA 3 mix $(5.0 \%)$, compared with that of the HMA 1 mix (4.5\%). Brittleness of the binder resulted in a higher stiffness in the HMA 3 mix at moderate and low temperature conditions. Stiffening of the binder used in the mix at low temperatures clearly influenced the response of the two mixes as only the binder rheological properties changed with temperature.

At Level 3 of the ASSHTO 2002 design procedure, no mechanical tests are required. Instead, the guide uses the model entitled "2000 Predictive Equation", discussed earlier in Chapter 3 , to estimate the dynamic modulus at different temperature conditions and loading frequencies (corresponding to traffic speeds). The estimated dynamic modulus values are then used to construct a master curve in a similar manner to that used in Level 1 of the design guide. Given the expected heavy use of Level 3 of the 2002 Design 
Guide, the predictive equations were examined using actual laboratory data produced using the testing protocol developed in this thesis and are further discussed in this chapter.

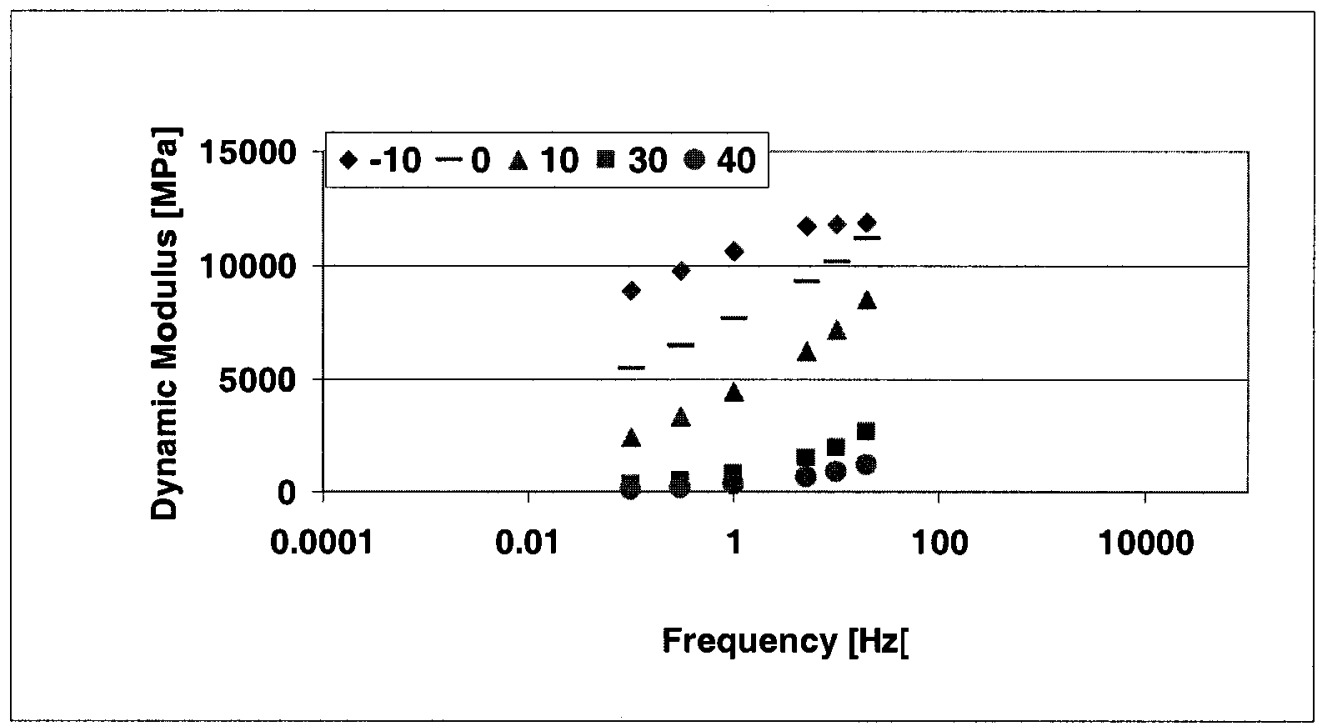

Figure 7.2. Measured laboratory data for the HMA 3 mix, PG 58-22

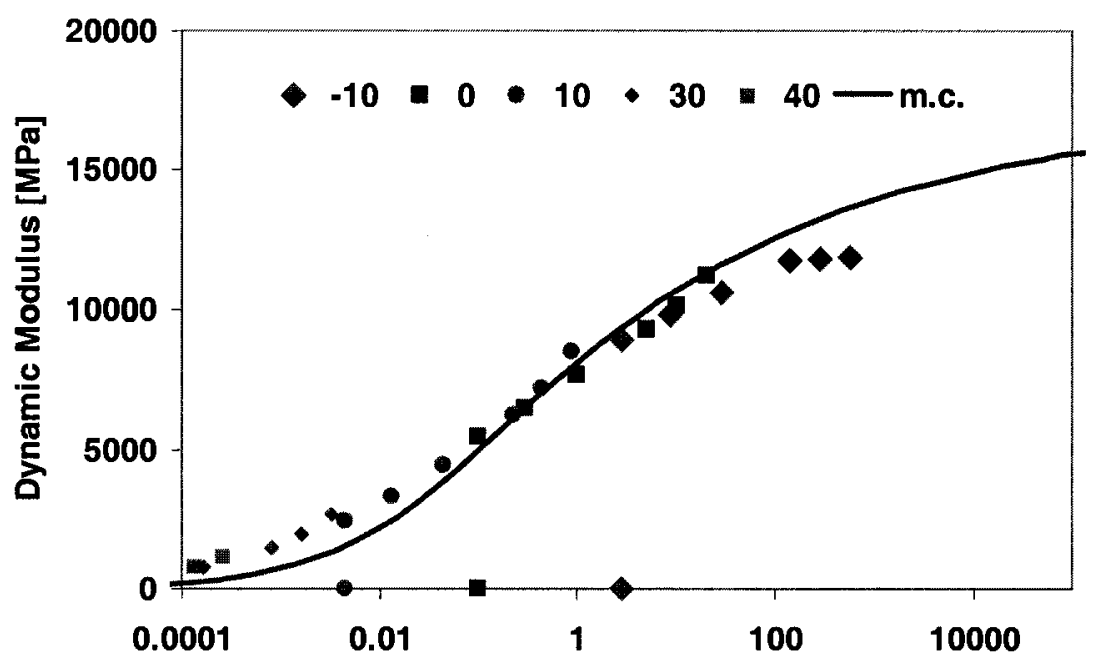

Frequency [Hz]

Figure 7.3. Master curve for the HMA 3 mix, PG 58-22 


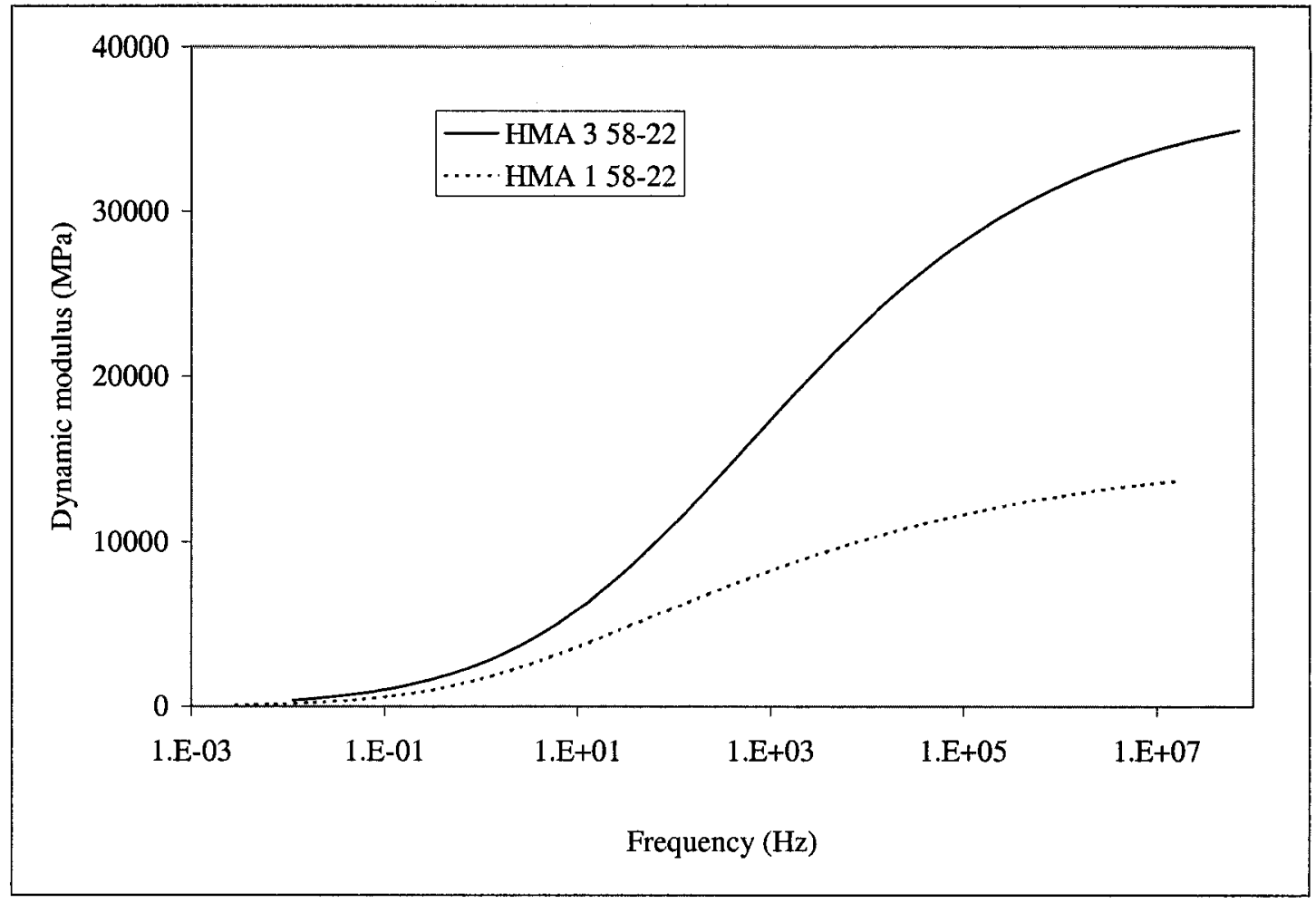

Figure 7.4. Master curves of two distinct HMA mixes

\subsection{Huet-Sayegh Rheological Model}

Application of the Huet-Sayegh rheological model was considered in this study because of its popularity in Europe and recent interest expressed by researchers in North America in employing the model in roadway pavement design and analysis. In the past, the HuetSayegh rheological model was implemented in finite element programs in which the correspondence principle was used to bring the problem from the time domain to the frequency domain, which can be summarized in four steps $[72,73]$ :

- Remove all time dependencies of the mechanical problem by transforming all timedependent variables to the frequency domain using Fourier transformation;

- Write the viscoelastic properties in terms of frequency; 
- Solve per frequency the obtained linear viscoelastic problem of the asphalt layer and obtain displacements, stresses and strains;

- Inversely transform the obtained displacements, stresses and strains for the AC layers from the Fourier to the time domain.

The Huet Sayegh model and its parameters were described in Chapter 3. The model consists of five parameters that are determined by obtaining the best fit for the measured dynamic modulus values plotted in the Cole-Cole and Black diagrams. The least square method is usually used to achieve the best fit. A computer program, HUSAROAD was used in this thesis to determine the eight parameters of the Huet-Sayegh model. Using the results of the developed complex modulus test protocol performed on an HMA 3 mix prepared with PG 52-34 binder, Figures 7.5 and 7.6 were constructed to show the fitting of laboratory data according to the Cole-Cole and Black diagrams. Three of the parameters $(\mathrm{a}, \mathrm{b}$ and $\mathrm{c}$ ) that describe the temperature dependence of this model were also determined by producing the best fit for the isothermal curves to assess variation in the characteristic time parameter with temperature as shown in Figure 7.7. The best fit that yielded the parameters $a, b$ and $c$ is shown in Figure 7.8. The Huet-Sayegh model parameters determined for some of the commonly used mixes evaluated in this thesis are shown in Table 7.3.

The data for two different mixes (HMA 1 and HMA 3) designed according to the same protocol (Marshall procedure) prepared using a PG 58-22 binder were plotted in a ColeCole diagram as shown in Figure 7.9. The plotted data reflects the ability of the test results presented according to the Huet-Sayegh rheological model to effectively discriminate between two mixes known to produce different responses and consequently 
performance. The range of the storage modulus of the HMA 3 is greater than that of the HMA 1, reflecting the impact of the binder content as discussed on the ASSHTO Design Guide application. A similar observation was made based on the results of the two SuperPave mixes used in this investigation (see Figure 7.10). Both of the SuperPave mixes had a binder content of about $5.0 \%$ and, as a result, their maximum storage moduli were comparable. Furthermore, when the curves of the SP 2 SuperPave mix and the HMA 3 Marshall mix with equivalent binder contents are examined, their responses are found to be comparable (See Figure 7.11).

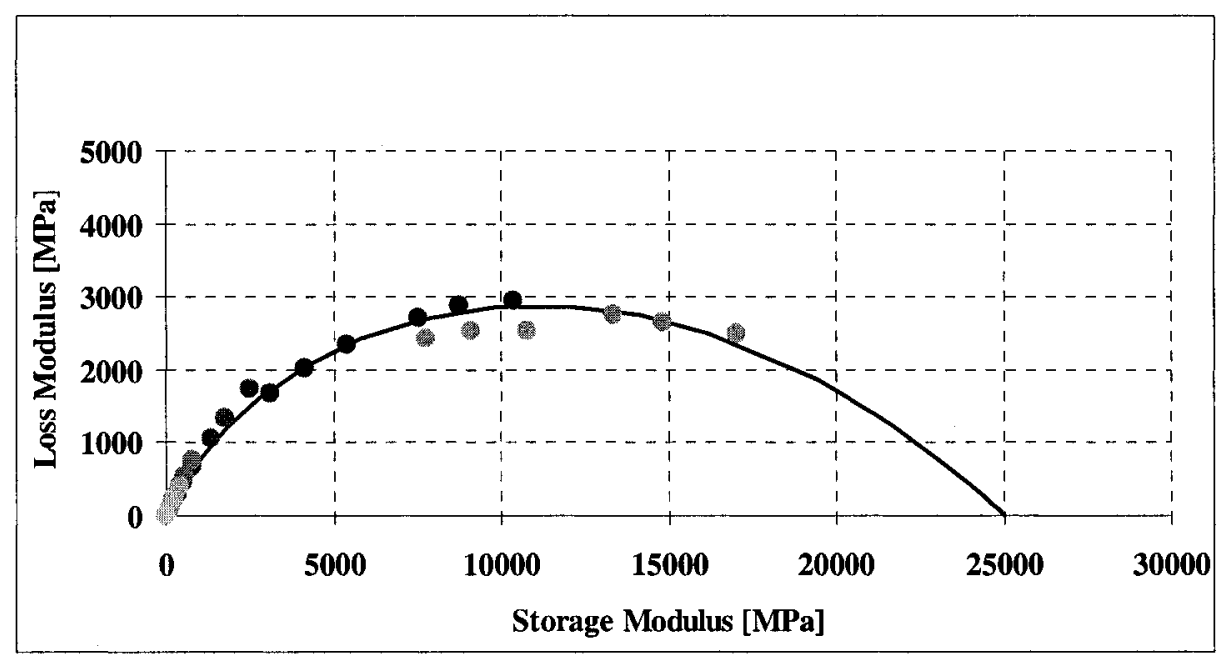

Figure 7.5. Fitting test results in the Cole-Cole diagram

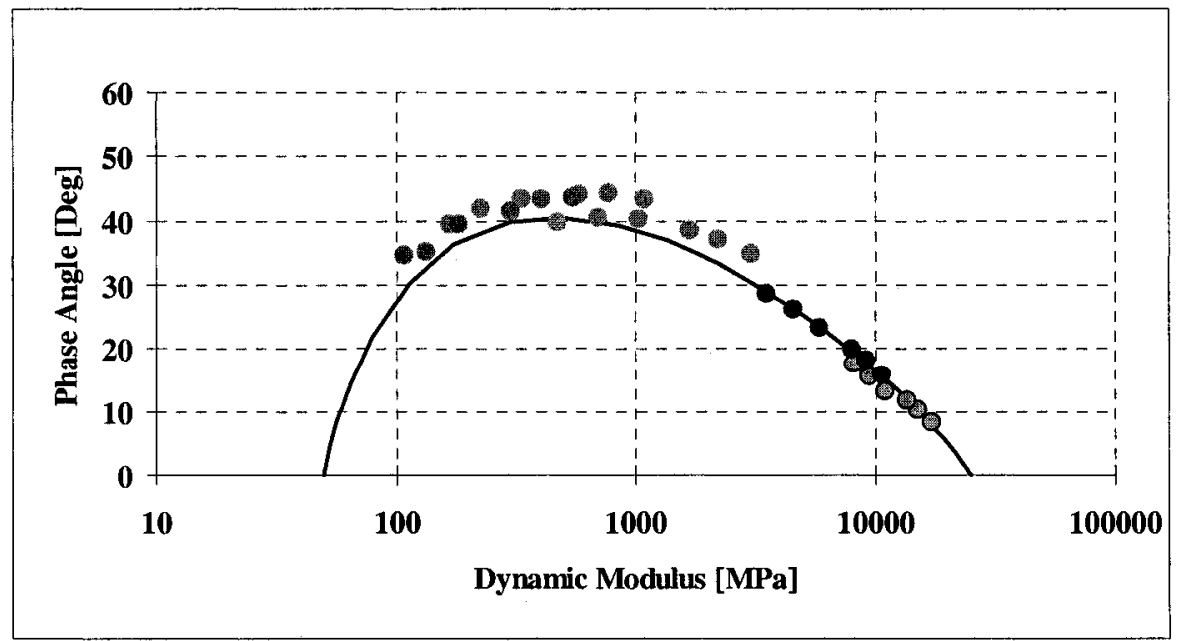

Figure 7.6. Fitting test results in the Black diagram 


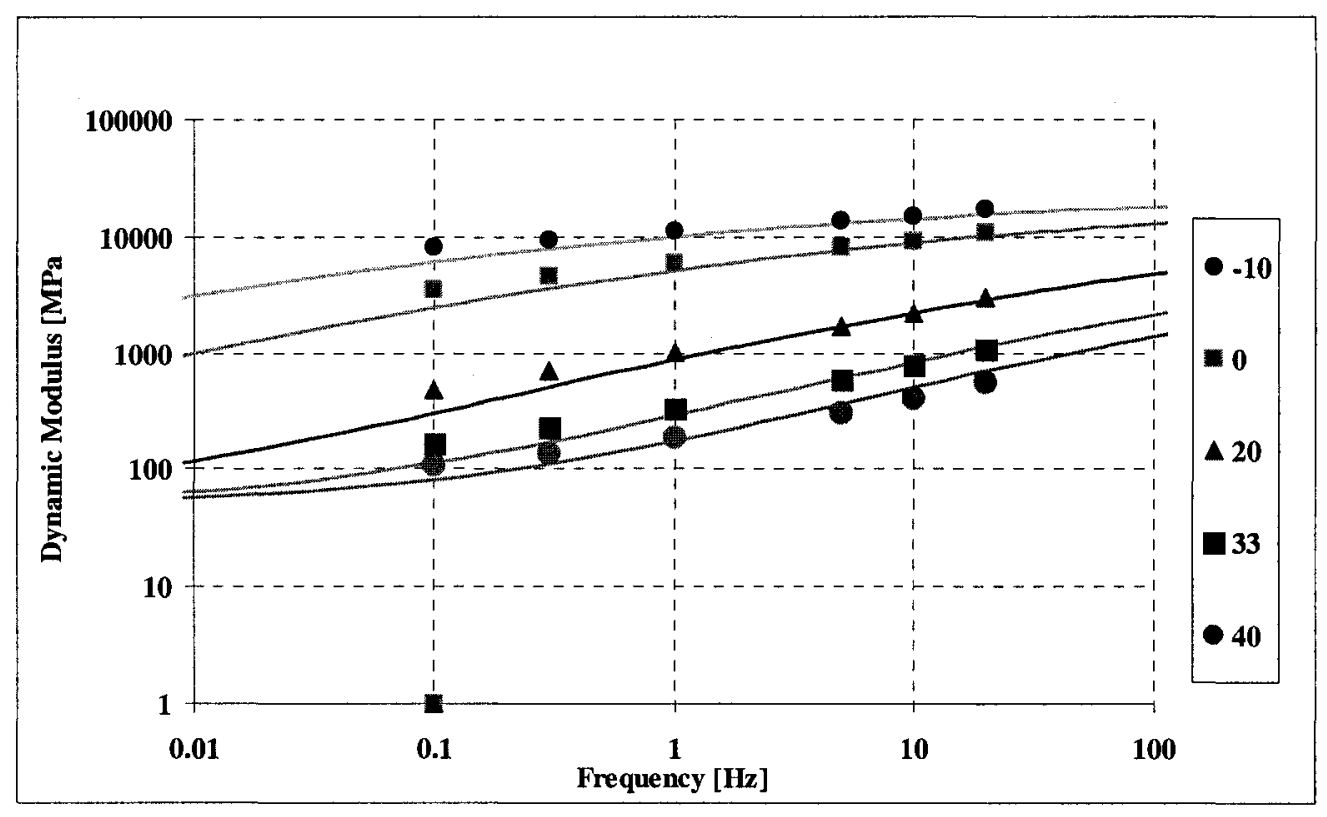

Figure 7.7. Fitting of isothermal curves

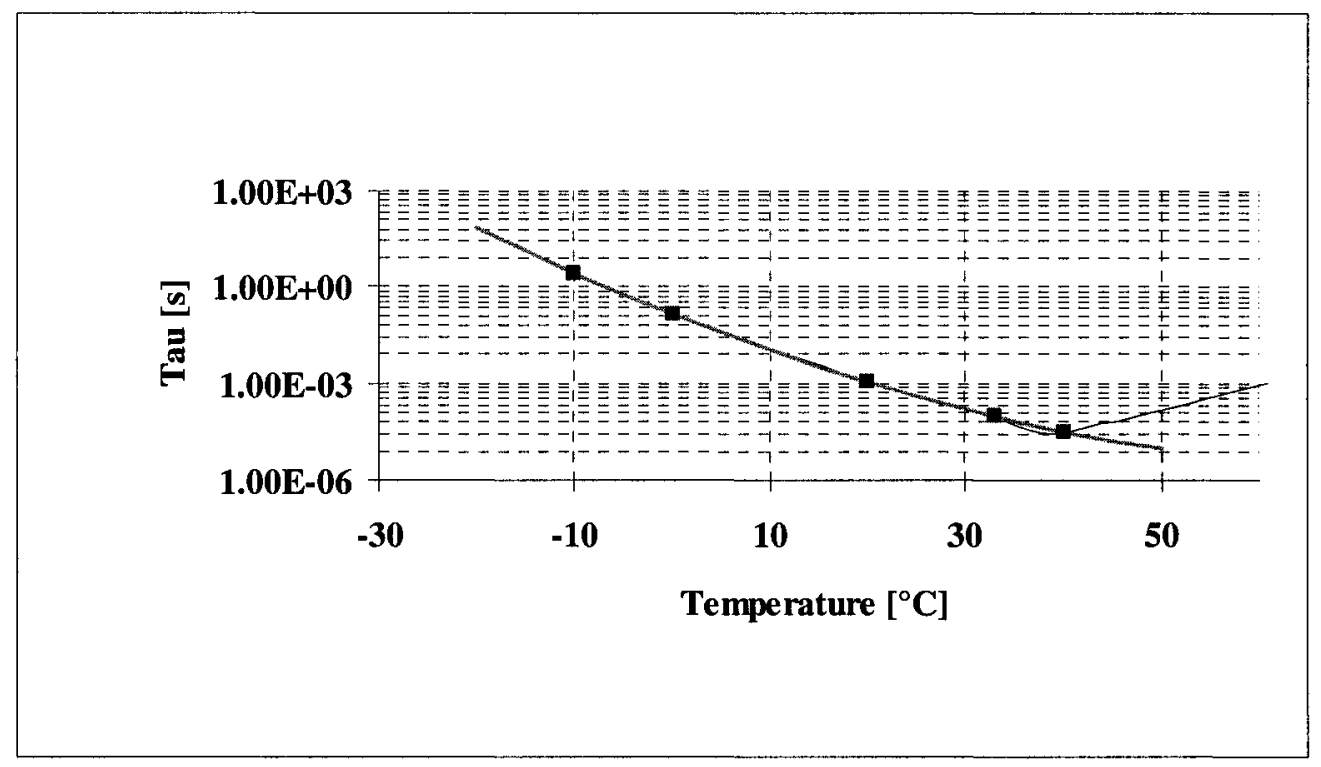

Figure 7.8. Fitting of the characteristic time parameter 


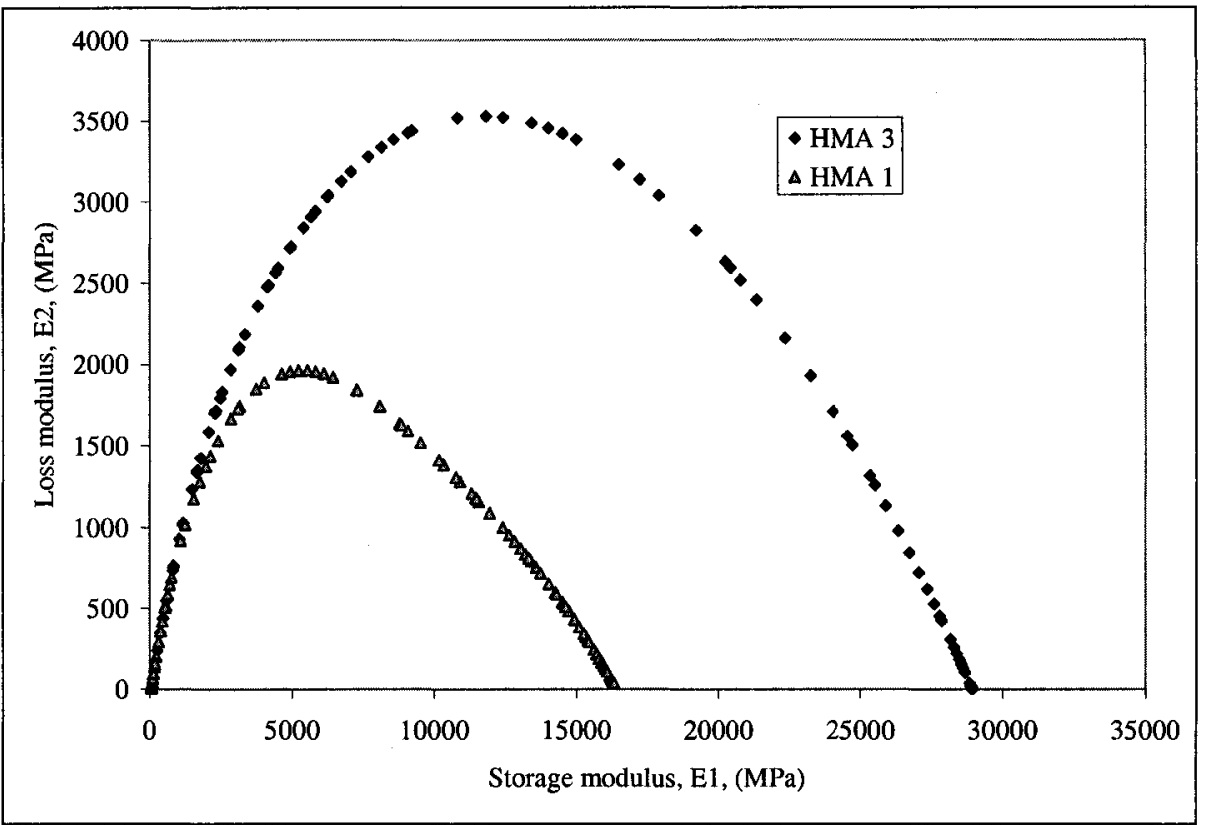

Figure 7.9. Cole-Cole diagram for two HMA mixes

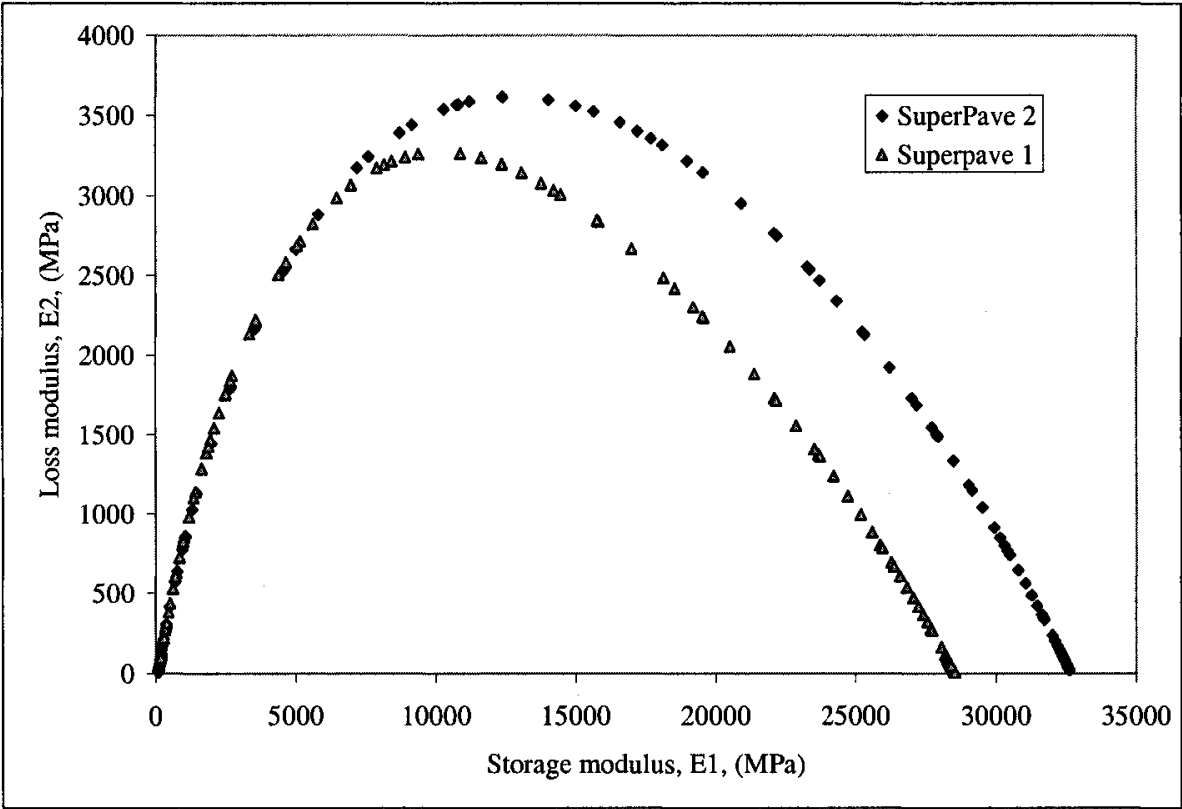

Figure 7.10. Cole-Cole diagram for two SuperPave mixes 


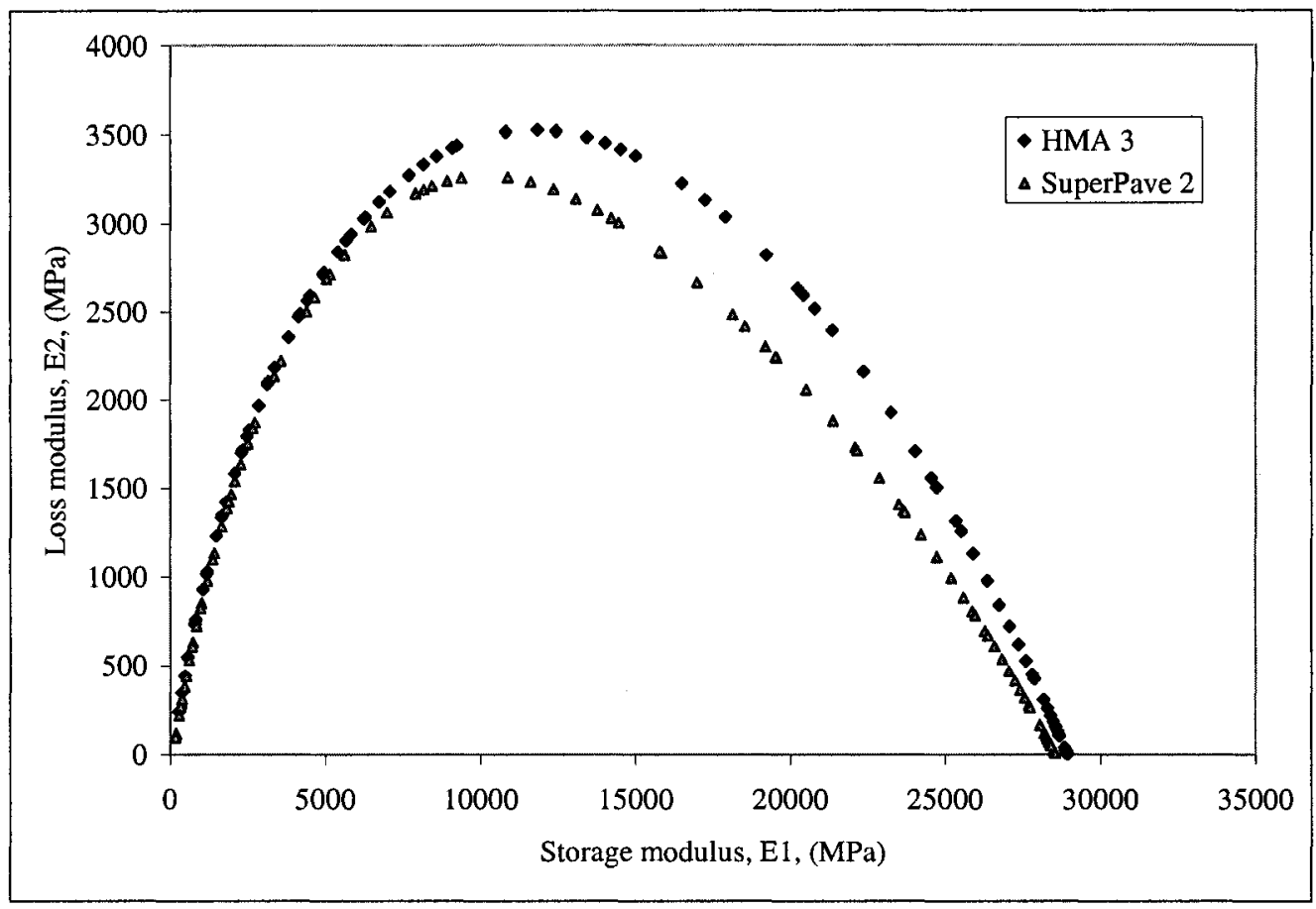

Figure 7.11. Cole-Cole diagram for similar HMA and SuperPave mixes

Table 7.3. Huet-Sayegh parameters for all mix designs tested in the thesis

\begin{tabular}{|l|l|l|l|l|l|l|l|l|}
\hline Sample ID & $\mathbf{E}_{\infty}$ & $\mathbf{E}_{\mathbf{0}}$ & $\delta$ & $\mathbf{k}$ & $\mathbf{h}$ & $\mathbf{a}$ & $\mathbf{b}$ & $\mathbf{c}$ \\
\hline HMA 3 64-34 & 23715 & 35 & 2.794 & 0.239 & 0.567 & -2.5975 & -0.3026 & 0.0017 \\
\hline HMA 3 58-22 & 28932 & 68 & 2.082 & 0.253 & 0.607 & 1.0699 & -0.3629 & 0.0042 \\
\hline HMA 3 52-34 & 24983 & 17 & 2.735 & 0.258 & 0.593 & -1.6586 & -0.2343 & 0.0001 \\
\hline HMA 2 64-34 & 17726 & 25 & 2.803 & 0.285 & 0.555 & -1.2744 & -0.2785 & 0.0008 \\
\hline HMA 2 58-22 & 36812 & 188 & 3.427 & 0.278 & 0.633 & 1.4931 & -0.3183 & 0.0012 \\
\hline HMA 2 52-34 & 24967 & 33 & 2.982 & 0.278 & 0.616 & -1.7651 & -0.2811 & 0.0012 \\
\hline HMA 1 58-22 & 16440 & 60 & 1.480 & 0.195 & 0.600 & 0.8677 & -0.2853 & 0.0010 \\
\hline HMA 1 52-34 & 26813 & 187 & 2.421 & 0.251 & 0.593 & -2.3998 & -0.3509 & 0.0029 \\
\hline SP 2 64-34 & 28373 & 127 & 2.318 & 0.229 & 0.531 & -0.3065 & -0.2653 & 0.0007 \\
\hline SP 2 58-22 & 32666 & 86 & 1.956 & 0.224 & 0.556 & 1.8326 & -0.3300 & 0.0011 \\
\hline SP 1 52-34 & 31441 & 59 & 2.580 & 0.294 & 0.591 & -0.9711 & -0.3605 & 0.0025 \\
\hline SP 1 58-22 & 28557 & 93 & 1.568 & 0.205 & 0.560 & 0.5859 & -0.3420 & 0.0030 \\
\hline SP 1 52-34 & 29466 & 34 & 2.424 & 0.276 & 0.597 & -1.7679 & -0.3502 & 0.0029 \\
\hline
\end{tabular}

\subsection{Predictive Equations}

Input parameters required for the predictive equations discussed earlier in Chapter 3 were obtained from the results of physical tests performed in this study are shown in Tables 4.19 through 4.23. Recommended values of the A and VTS coefficients are given in 
Table 7.4 [71]. Dynamic modulus predictions produced using the two equations considered in this study for different test temperatures and loading frequencies were compared with results from mechanical tests using the test protocol developed in this thesis. A comparison between the predicted and measured dynamic modulus was performed at three different temperatures $\left(-10,20\right.$ and $\left.40^{\circ} \mathrm{C}\right)$. These test temperatures were chosen to represent cold, moderate and warm operating temperatures. The selected loading frequencies $(0.1,1$ and $20 \mathrm{~Hz})$ represent slow, medium and relatively fast traffic conditions. To fully assess the capabilities of the predictive equations and delineate their strong and weak points, the results of the complex modulus laboratory experiments and the outcomes of the predictive equations were grouped and analyzed from different perspectives to examine the influence of the parameters discussed below.

\subsubsection{Binder Effect}

The absence of a clear viscosity-temperature relationship was the main obstacle to the development of accurate predictive equations. This relationship is critical for accurately assessing the rheological behaviour of binders acting as part of the AC mix. In this study, the ability of the predictive equation to discriminate between different binders was evaluated. Since binders are graded at high and low service temperatures, the ability of predictive equations to detect the difference between the responses of these binders used in $\mathrm{AC}$ mixes represents an important evaluation criterion. The low temperature indicator $(-34)$ was the same for the two binder types. A comparison between the predicted values based on the 1996 version and the complex modulus test results is shown in Figure 7.12, using the equality line drawn at $45^{\circ}$. 


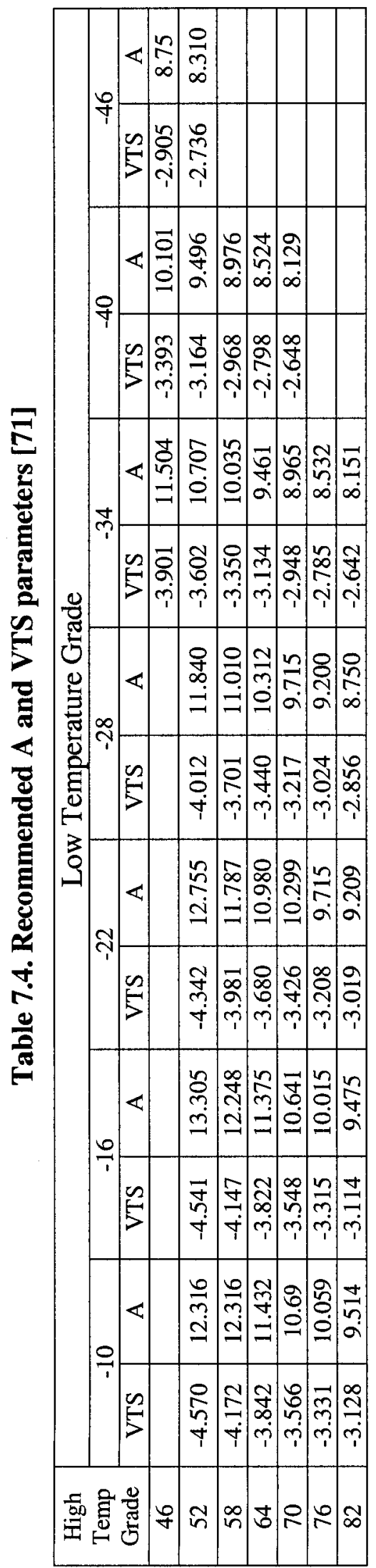


Points located above this line indicate that the predictions over-estimated the dynamic modulus value. Points below the equality line indicate under-estimated values. Actual coordinates of data points (measured, predicted) were used in this study to quantify deviation of predicted values from that measured in the laboratory. Lines that represent different percentages of deviations were used on both sides of the equality line to highlight deviation determined under different conditions. For example, a point falling between the equality line and the 0.8 Line represents less than $20 \%$ deviation in the form of under-estimation. Predictions made with the 1996 equation for the HMA 3 64-34 and HMA 3 52-34, shown in Figure 7.12, over-estimated dynamic modulus values except for the conditions that lead to high modulus values (higher than $13000 \mathrm{MPa}$ ). Under such conditions (mainly a combination of low test temperatures and high loading frequencies), the predictive equation has under-estimated the response by less than $20 \%$.

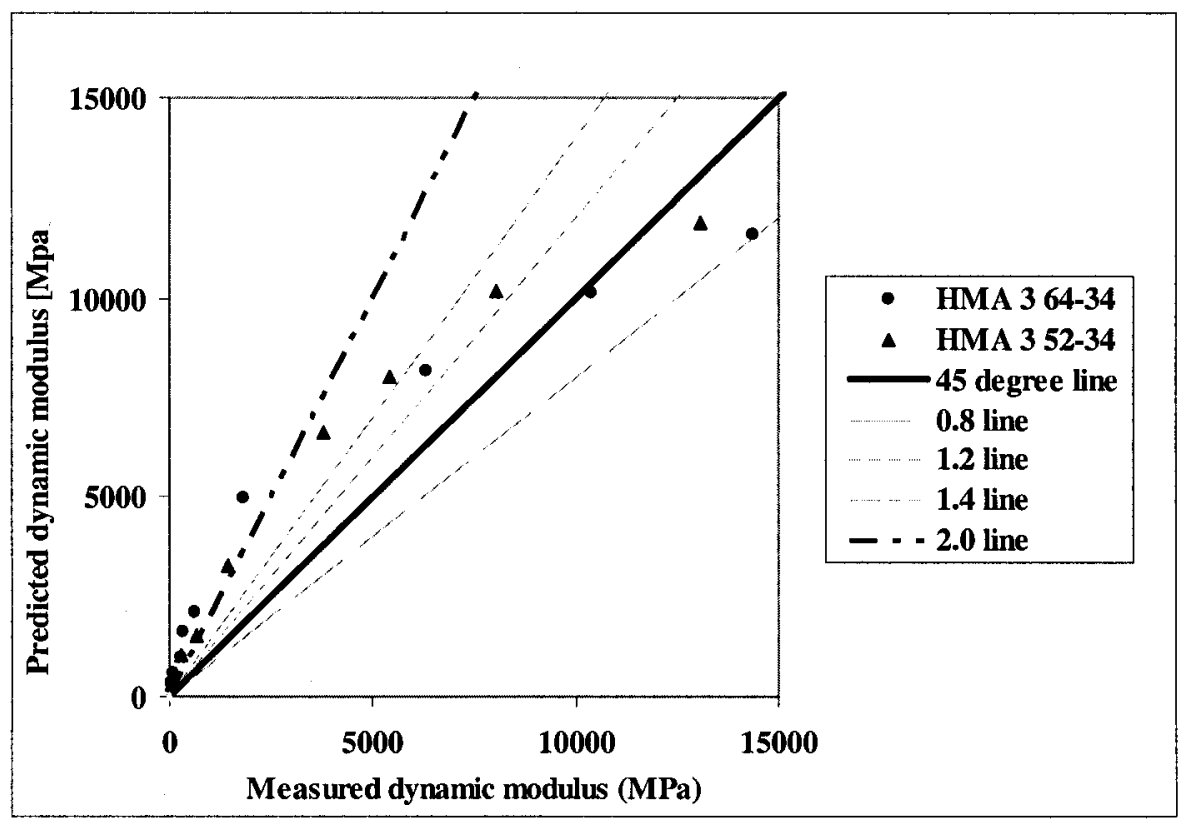

Figure 7.12. Evaluation of predictions made with 1996 equation for HMA mixes with different high-temperature binder grades 
Furthermore, at low modulus values, the two curves that represent the two mixes are close to each other, suggesting that the predictive equation does not discriminate between the two binder grades that are supposed to produce different levels of stability at high service temperatures. The same observations apply to mixes prepared according to SuperPave mix design (SP 2 64-34 and SP 2 52-34) at low to medium dynamic values (less than $5000 \mathrm{MPa}$ ) as shown in Figure 7.13.

The results from the 2000 predictive equation (Equation 3.28) applied to the Marshall mix designs produced a slight overall improvement as shown in Figure 7.14. The deviation in modulus predictions applied to the HMA mix was smaller than the deviation encountered when the 1996 equation was used, especially at high dynamic modulus values (a deviation of less than 40\%). A similar slight improvement was also apparent when results of the SuperPave mix design mixtures (see Figure 7.15) were examined. Most of moduli predictions at values higher than $1000 \mathrm{MPa}$ deviated from the actual measurements by less than $30 \%$. 


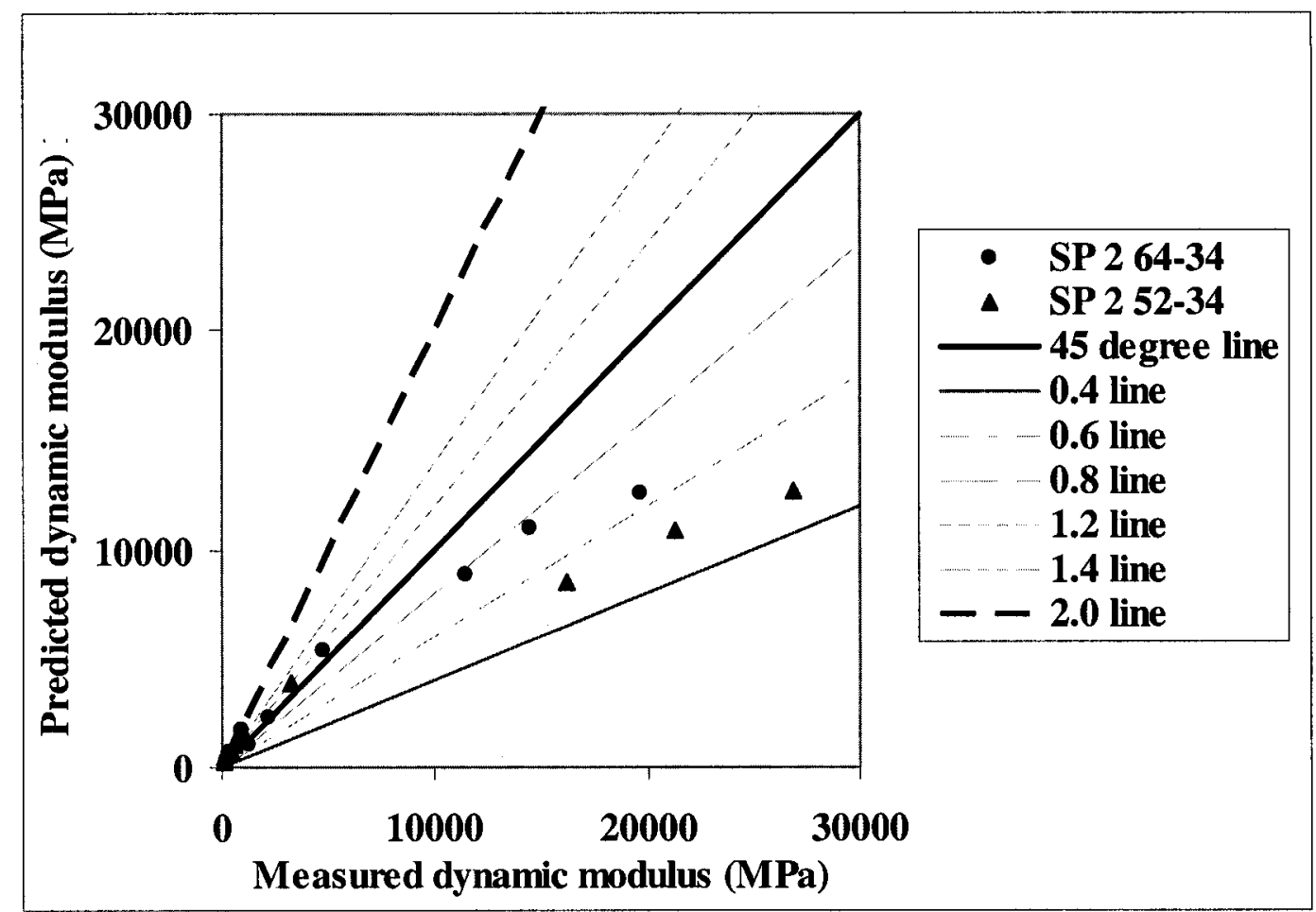

Figure 7.13. Evaluation of predictions made with 1996 equation for SuperPave mixes with different high-temperature binder grades

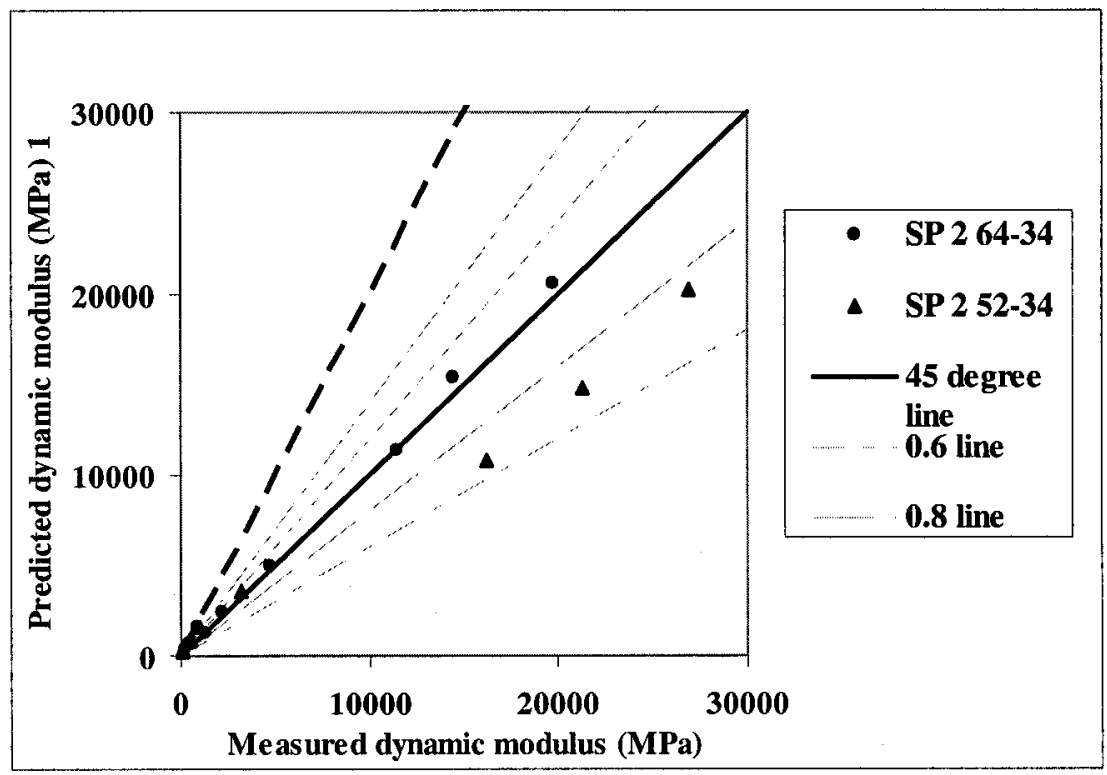

Figure 7.14. Evaluation of predictions made with 2000 equation for HMA mixes with different high-temperature binder grades 


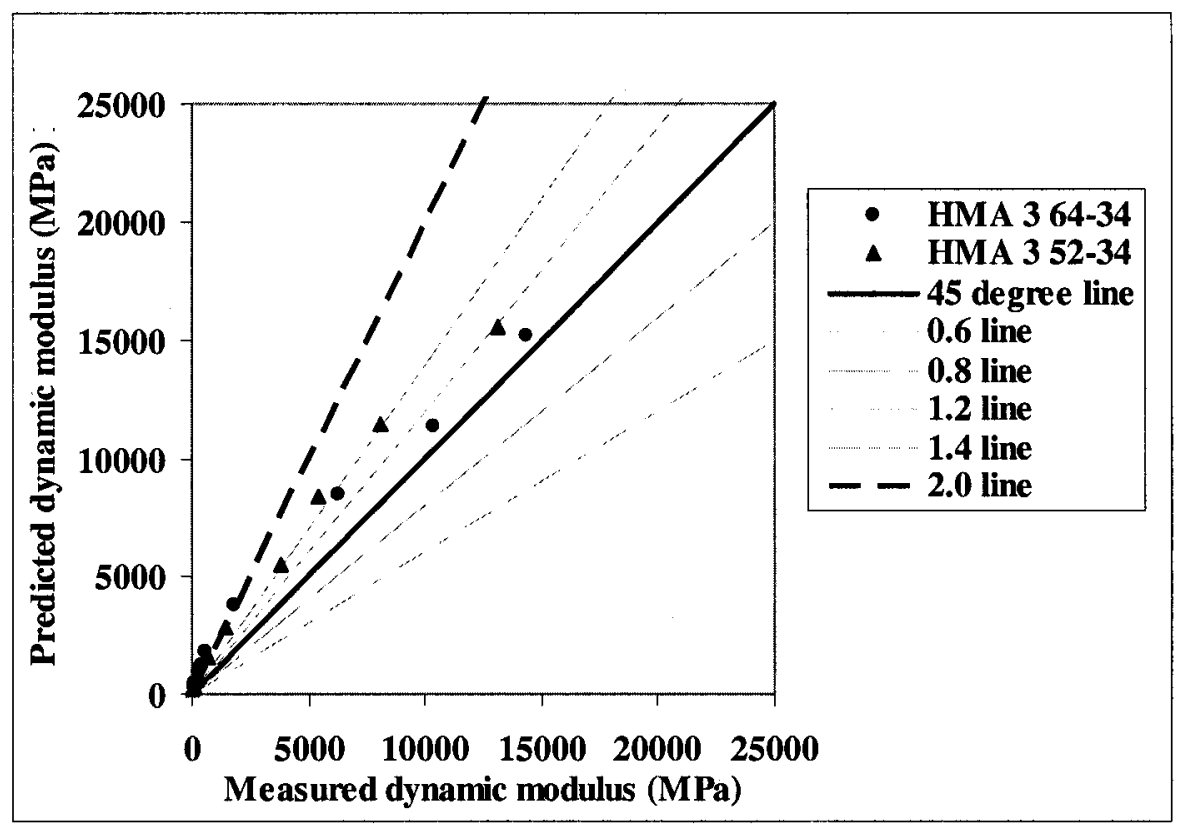

Figure 7.15. Evaluation of predictions made with 2000 equation for SuperPave mixes with different high-temperature binder grades

Further analysis of the effect of binder grade at high service temperatures on the accuracy of the predictive equations was undertaken. Considering the HMA 3 prepared with the PG 64-34 and 52-34 binders discussed above, predictions made using the 1996 equation for the three test temperatures were plotted in Figure 7.16. The results conform with the trend presented above. For a high temperature $\left(40^{\circ} \mathrm{C}\right)$, the predictive equation did not discriminate between the two mixes, which had very different responses at high temperatures (high stability for the PG 64-34 compared to the PG 52-34). The predictions made at moderate temperatures, represented by the $20^{\circ} \mathrm{C}$ test temperature, also showed little distinction between the two grades. It has been argued in the literature that at high temperatures the stiffness of the aggregate skeleton is the major contributor to the mix stiffness and that the binder plays a minor role. The 1996 predicted results agree with this observation. At moderate temperatures (between 35 and $5^{\circ} \mathrm{C}$ ), the binder starts to contribute more to the stiffness as seen in Figure 7.16. The same trend was also apparent 
when the results of the 2002 predictive equation were plotted (see Figure 7.17). This behaviour was also confirmed by the measured dynamic modulus as shown in Figure 7.18. The measured dynamic moduli were identical for both binders at a high temperature $\left(40^{\circ} \mathrm{C}\right)$, and the deviation between the two mixes started to increase with decreasing temperature (from 35 to $5^{\circ} \mathrm{C}$ ).

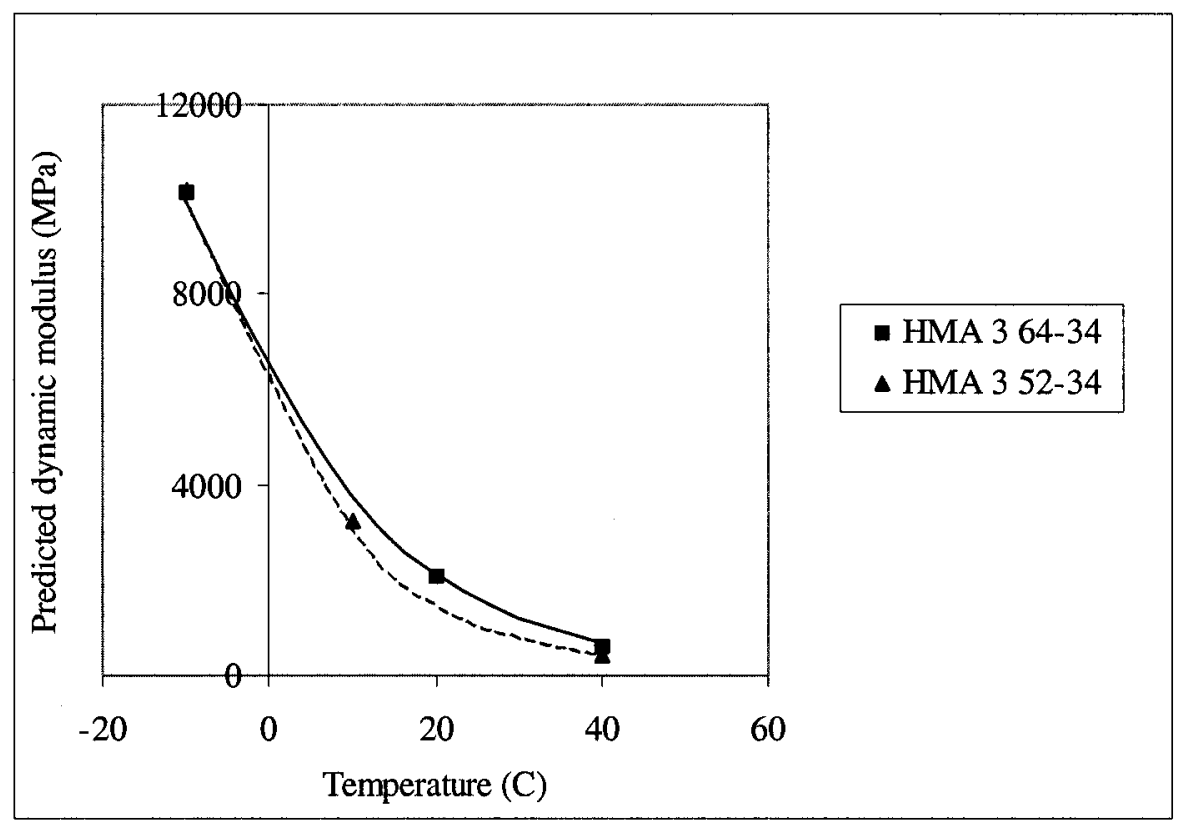

Figure 7.16. Predicted dynamic modulus vs. temperature - 1996 equation

Although the comparison was focused on the high temperature range, it is worth noting that measurement of the dynamic modulus in the laboratory showed a $29 \%$ difference between the two mixtures at low temperatures. The absence of such a difference applies to both predictive equations as reflected in the dynamic modulus values predicted for $10^{\circ} \mathrm{C}$ as shown in Figures 7.16 and 7.17. Based on the test results, measures built into the two predictive equations automatically assume that the two binders will have similar lowtemperature responses. 


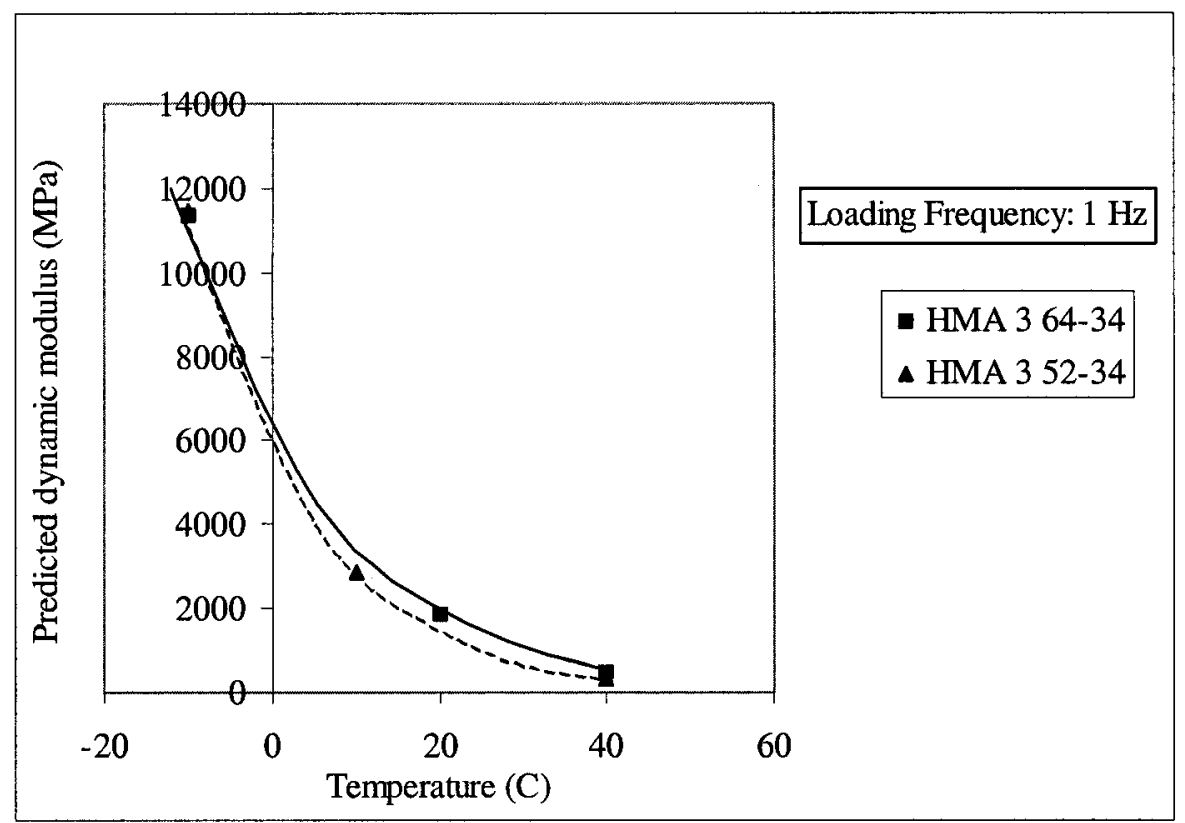

Figure 7.17. Predicted dynamic modulus vs. temperature - 2000 equation

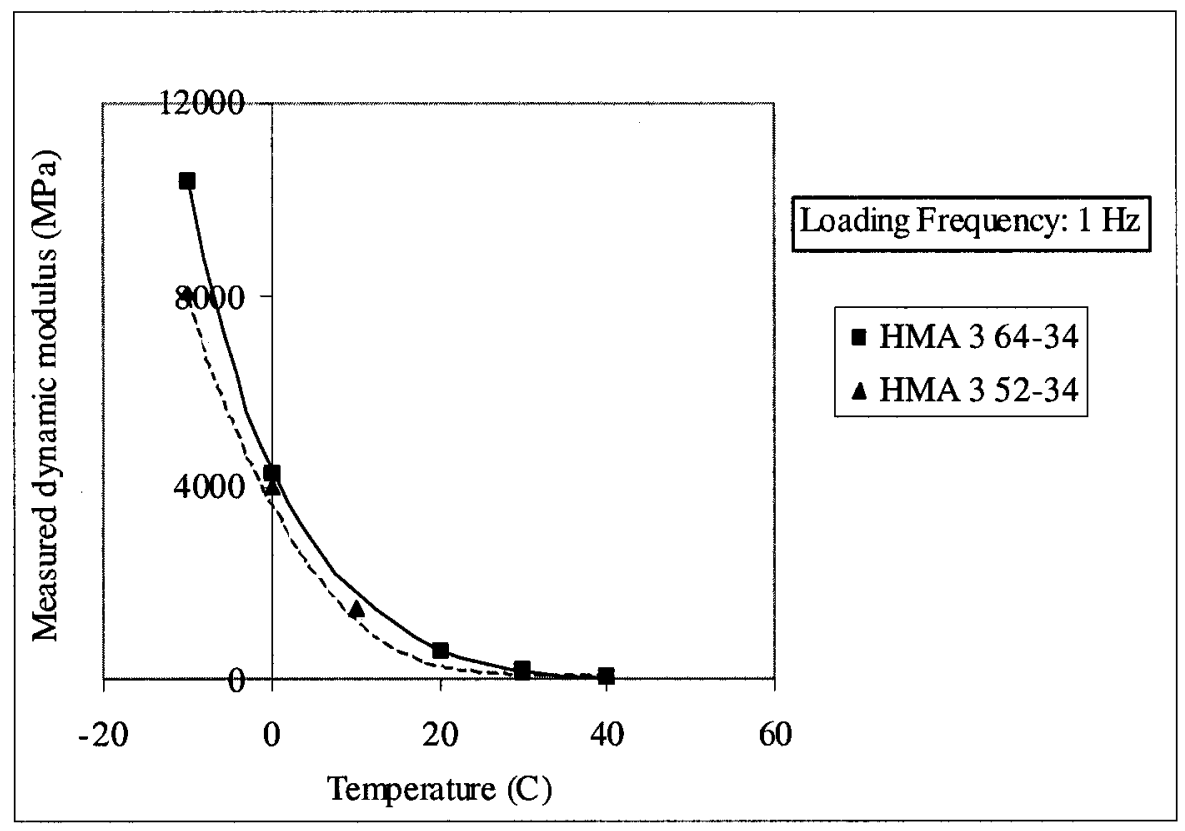

Figure 7.18. Measured dynamic modulus vs. temperature

Outcome of statistical analysis performed on the results of the tests performed on the HMA 3 mixes prepared with these two different performance grade binders (PG 64-34 and 52-34), presented in Table 7.5. The predictions confirmed that the response of the 
two mixes was similar. These results highlight the improvement brought by application of the 2000 equation. The two mixes reflected comparable average percent errors of more than $200 \%$ for the 1996 equation and an average of $168 \%$ for the 2000 equation.

Table 7.5. Results of statistical analysis

\begin{tabular}{|c|c|c|}
\hline & \multicolumn{2}{|c|}{ Average Percent Error (\%) } \\
\hline Equation & HMA 3 64-34 & HMA 52-34 \\
\hline 1996 & 232 & 255 \\
\hline 2000 & 169 & 167 \\
\hline
\end{tabular}

The assessment of predictions made for two mixes prepared with two different lowtemperature grades followed the same line of analysis undertaken for the hightemperature side. For this purpose, predicted (using the 1996 equation) and the measured modulus values for the HMA 3 58-22 and HMA 3 64-34 mixes were plotted around the $45^{\circ}$ equality line in Figure 7.19. The HMA 3 64-34 results indicate a trend towards overprediction compared with the measured values, except for the high values for which the modulus was under-predicted by more than $20 \%$. In contrast, estimates of the (HMA 3 , PG 58-22 binder) modulus were mainly under-predicted (with a deviation of up to about $50 \%$ ), except in the case of small modulus values for which over-predictions of less than $20 \%$ were observed. The results of the 2000 prediction equation were plotted in Figure 7.20. The predictions appear to be better than those of the 1996 equation as the deviations from the equality line were lower. 


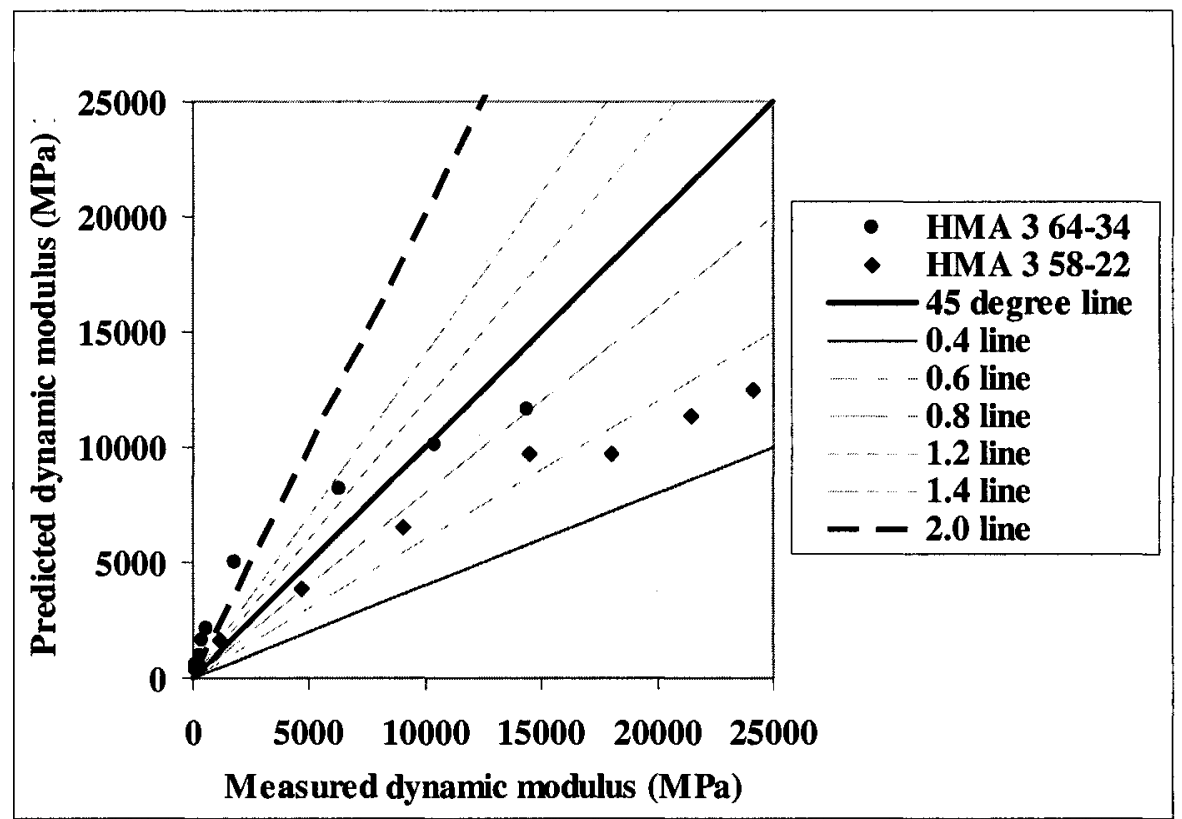

Figure 7.19. Evaluation of predictions made with 1996 equation for HMA mixes with different low-temperature binder grades

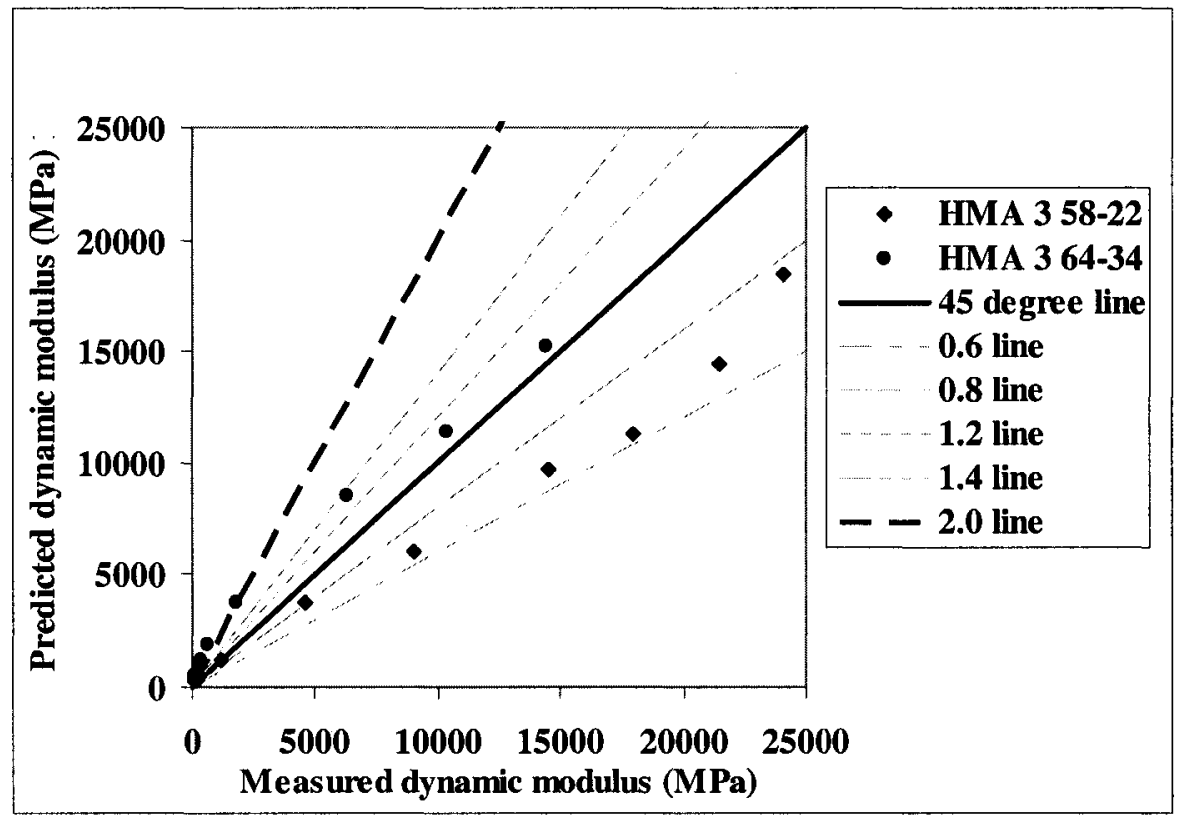

Figure 7.20. Evaluation of predictions made with 2000 equation for HMA mixes with different low-temperature binder grades

Statistical analysis, performed on the data pertaining to the two mixes presented in Table 7.5 , confirmed that the overall improvement brought by the 2000 equation in terms of 
lower mean absolute error and average percent error, gave a clearer picture of the effect of binder type at low temperature conditions. The two predictive equations possess limited capabilities for predicting the dynamic modulus of mixes with engineered binders as reflected in the high average percent errors (223\% for the 1996 equation and $169 \%$ for the 2000 equation). However, predictions of the response for the mix with a conventional binders (PG 58-22) using the two equations, were much better as indicated by lower average percent errors given in Table 7.6.

Table 7.6. Statistical analysis results

\begin{tabular}{|c|c|c|c|c|}
\hline \multirow{2}{*}{ Equation } & \multicolumn{2}{|c|}{ Mean Absolute error (MPa) } & \multicolumn{2}{c|}{ Average Percent Error (\%) } \\
\cline { 2 - 5 } & HMA 3 64-34 & HMA3 58-22 & HMA 3 64-34 & HMA 3 58-22 \\
\hline 1996 & 1363 & 4332 & 223 & 56 \\
\hline 2000 & 1024 & 3159 & 169 & 37 \\
\hline
\end{tabular}

The impact of the binder grade on the mix response at low temperatures was further analyzed in a similar way to that followed in the analysis of high temperatures. The dynamic modulus values at different temperatures are shown in Figure 7.21, 7.22 and 7.23 for the 1996 predictive equation, the 2000 predictive equation, and the test results, respectively. The results shown in these three figures indicate that the two predictive equations managed to correctly rate the response at low temperatures associated with the two binders. However, these two predictive equations under-estimated the difference between the two binders as quantified in the test results. The 1996 predictive equations showed a difference of $12 \%$ compared with the measured response, which reflects a $100 \%$ difference. The 2000 predictive equation showed a slight improvement in which the difference between the mixes was $27 \%$. 


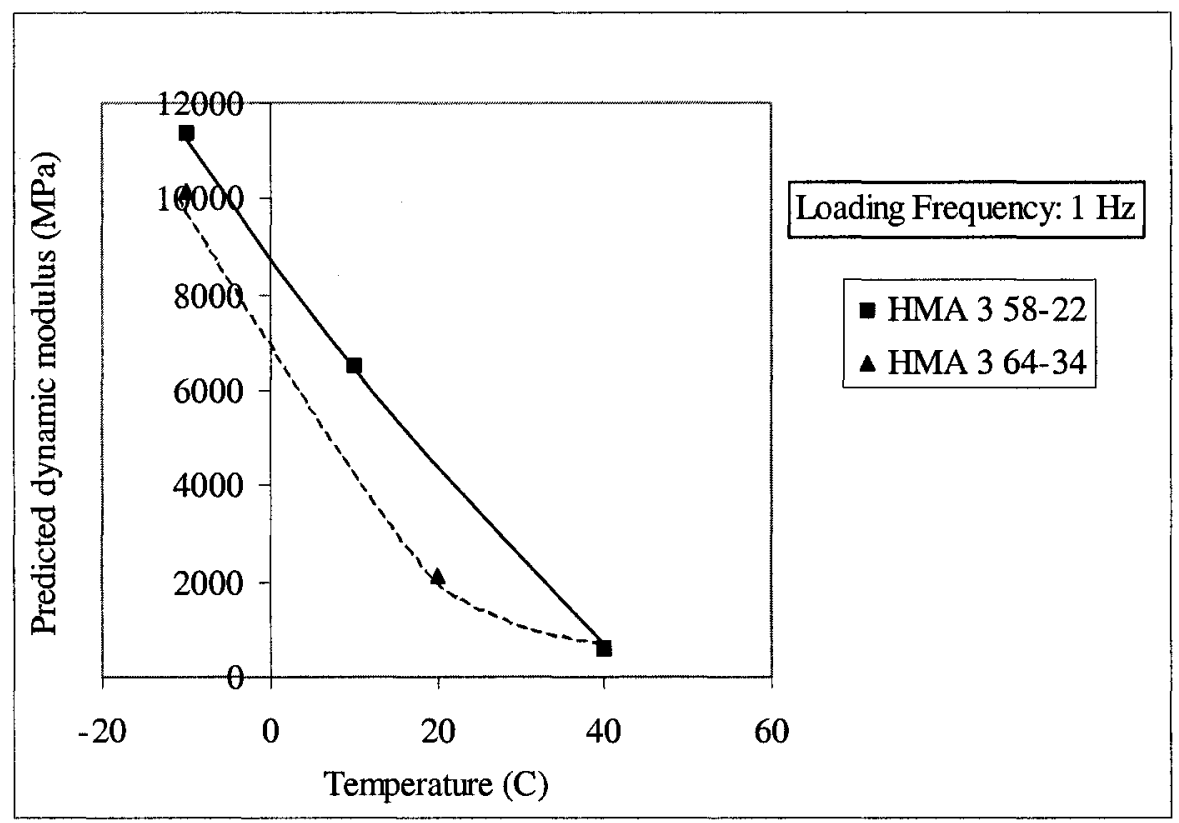

Figure 7.21. Predicted dynamic modulus vs. temperature - 1996 equation

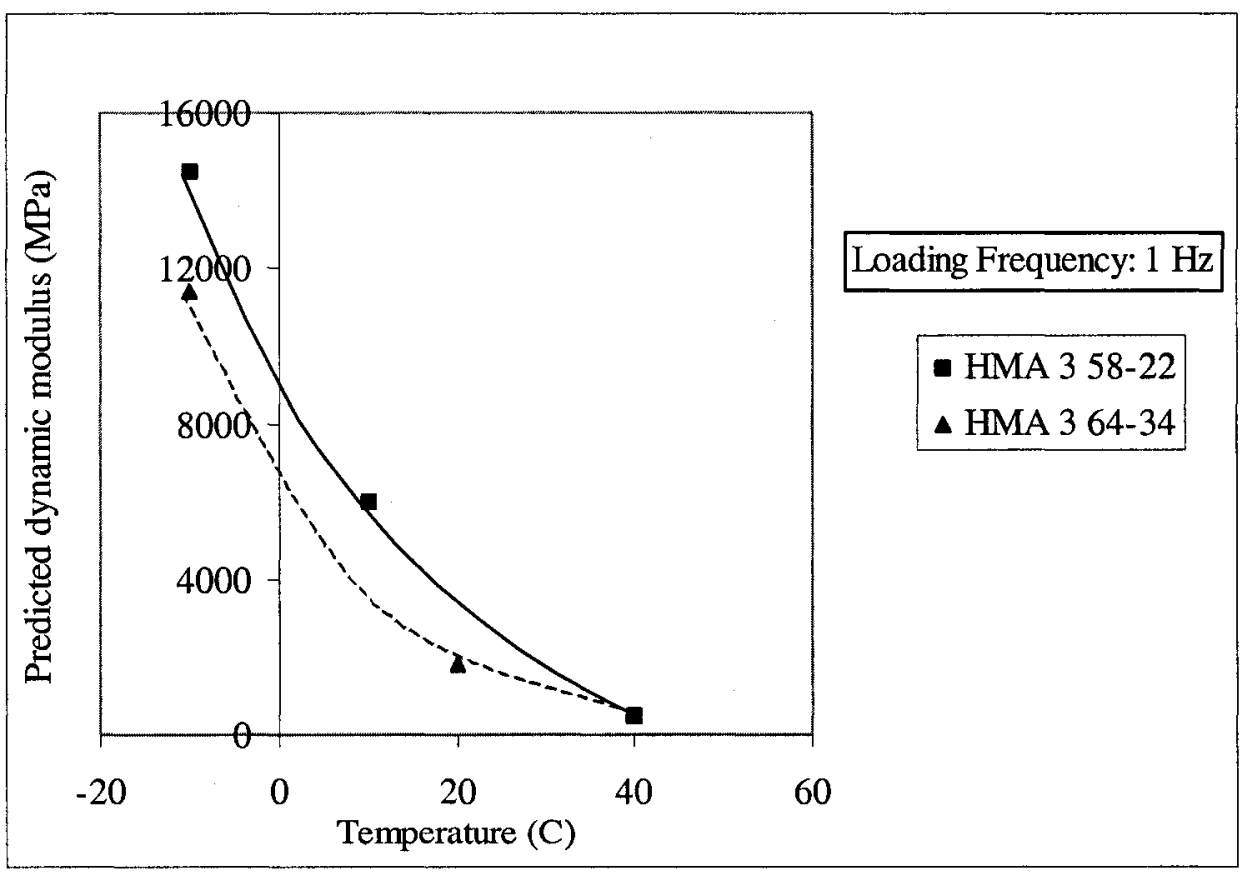

Figure 7.22. Predicted dynamic modulus vs. temperature - 2000 equation 


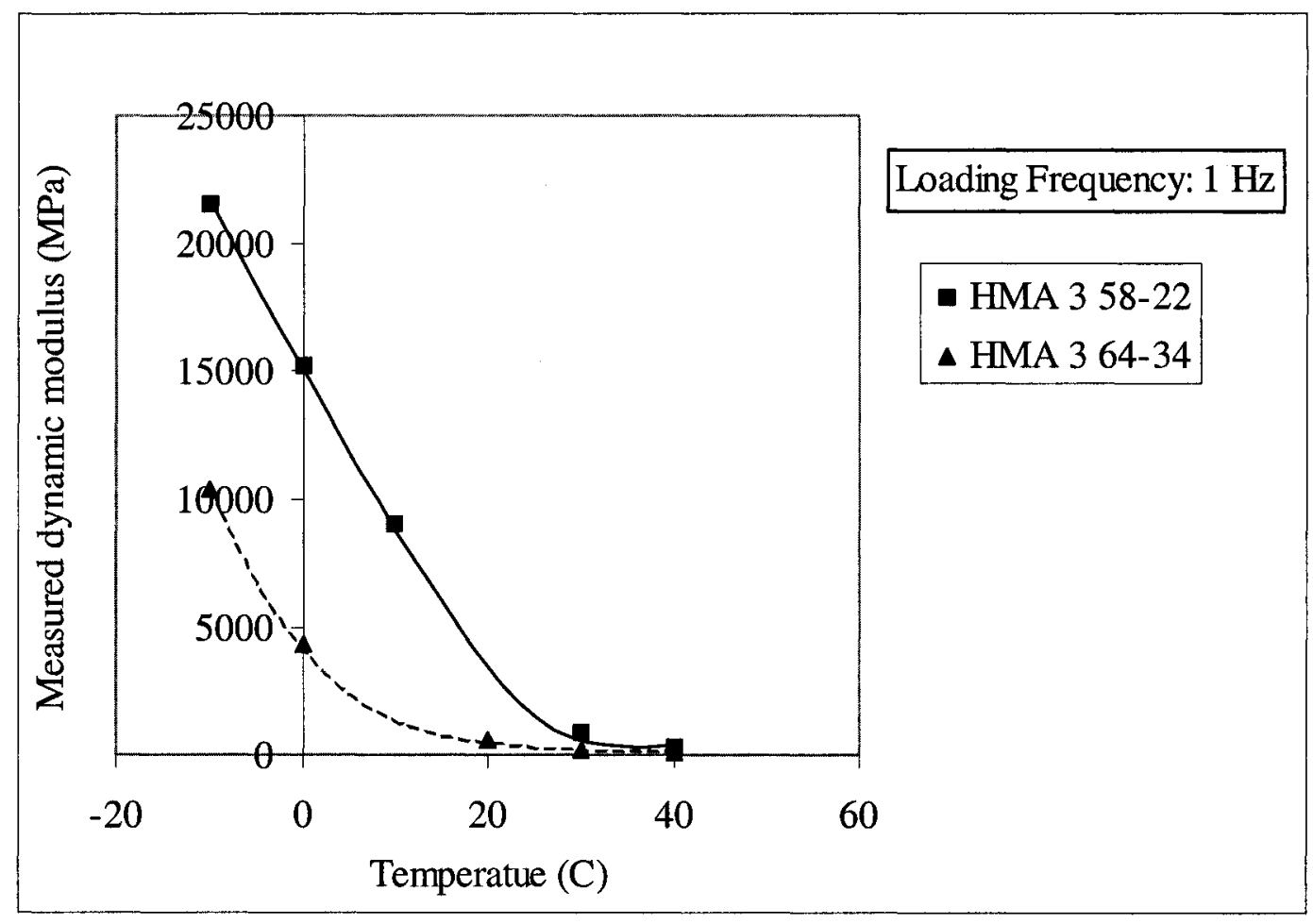

Figure 7.23. Measured dynamic modulus vs. temperature

It is clear from the test results that the engineered binder (PG 64-34) will fulfill its purpose, which aims for flexibility by reducing brittleness at low temperatures, hence reducing the potential for cracking. The measured dynamic modulus of the HMA 3 with PG 64-34 is half the value of the HMA 3 with PG 58-22 binder. Both measured and predicted dynamic moduli (using the two predictive equations) of the two binders are identical at high temperatures, reinforcing observations made earlier about the roleplayed by the aggregate skeleton at high temperatures.

\subsubsection{Temperature Effect}

To evaluate the capabilities of the predictive equations as influenced by the variations in test temperatures, the measured and predicted dynamic moduli of all mixtures were compared. The results using the 1996 equation are shown in Figures 7.24, 7.26 and 7.28, 
with predictions and the results of actual tests performed at $-10,20$ and $40{ }^{\circ} \mathrm{C}$, respectively. Figures $7.25,7.27$ and 7.29 provide the results of the 2000 equation evaluated in a similar fashion to the above comparative analysis.

At a low temperature $\left(-10^{\circ} \mathrm{C}\right)$, the majority of the predictions made with the 1996 equation fall under the $45^{\circ}$ line, suggesting that the modulus was under-estimated when compared with the test data produced from actual laboratory measurements (see Figure 7.24). The deviation of the predicted values from the measured ones increases with higher dynamic moduli. For moduli lower than $12500 \mathrm{MPa}$, the under-estimation by the 1996 equation was 20 to $40 \%$. This range shifted to 40 to $60 \%$ for moduli higher than $17000 \mathrm{MPa}$. When the 2000 equation was used, dynamic moduli predicted at a low temperature $\left(-10^{\circ} \mathrm{C}\right)$ showed significant improvement (see Figure 7.25$)$. The deviation observed earlier in the predictions made with the 1996 equation has now been balanced between under- and over-estimation by the 2000 equation, and the data points are now located over and under the 45 degree equality line. The increase in deviation with the increase in moduli observed in the 1996 predictions is now consistent at less than $40 \%$ of under- and over-estimation, with the exception of a few data points for which overprediction reaches $100 \%$ (2.0 line).

The picture changed for the predictions made at a moderate temperature of $20^{\circ} \mathrm{C}$ using the 1996 equation in which $40 \%$ of the data points reflected under-estimation and $60 \%$ over-estimation (see Figure 7.26). Except for occasions in which the dynamic moduli were higher than $7000 \mathrm{MPa}$, the under-estimated points fall below the $20 \%$ deviation $(0.8$ line). However, the over-predicted points fall beyond the $20 \%$ range (1.2 line) and beyond the $100 \%$ line ( 2.0 line) especially for dynamic modulus values lower than 1000 
MPa. Switching from the 1996 to the 2000 equation, dynamic moduli predictions at a moderate temperature did not show any significant change (see Figure 7.27). All previous observations concerning the 1996 equation hold for the predictions made with the 2000 model.

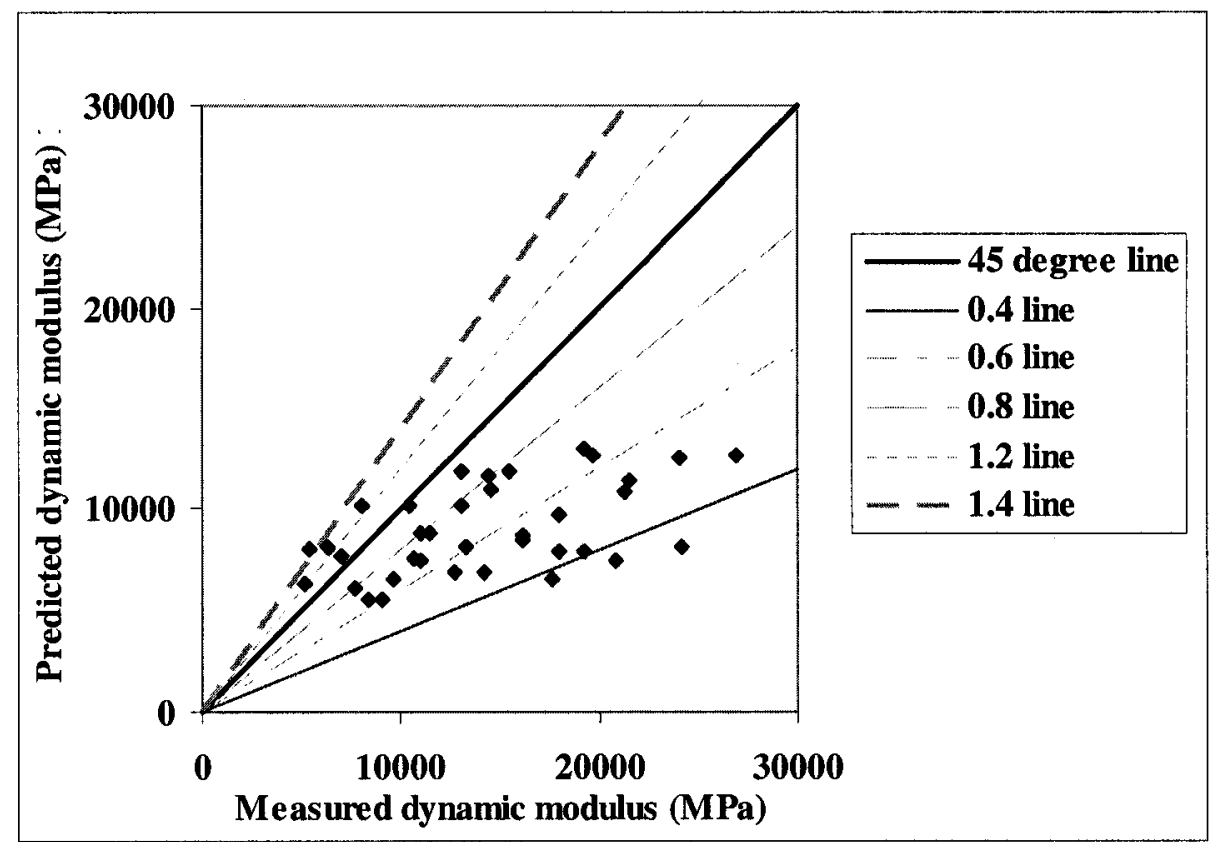

Figure 7.24. Dynamic modulus predictions at $-10^{\circ} \mathrm{C}$ using 1996 equation

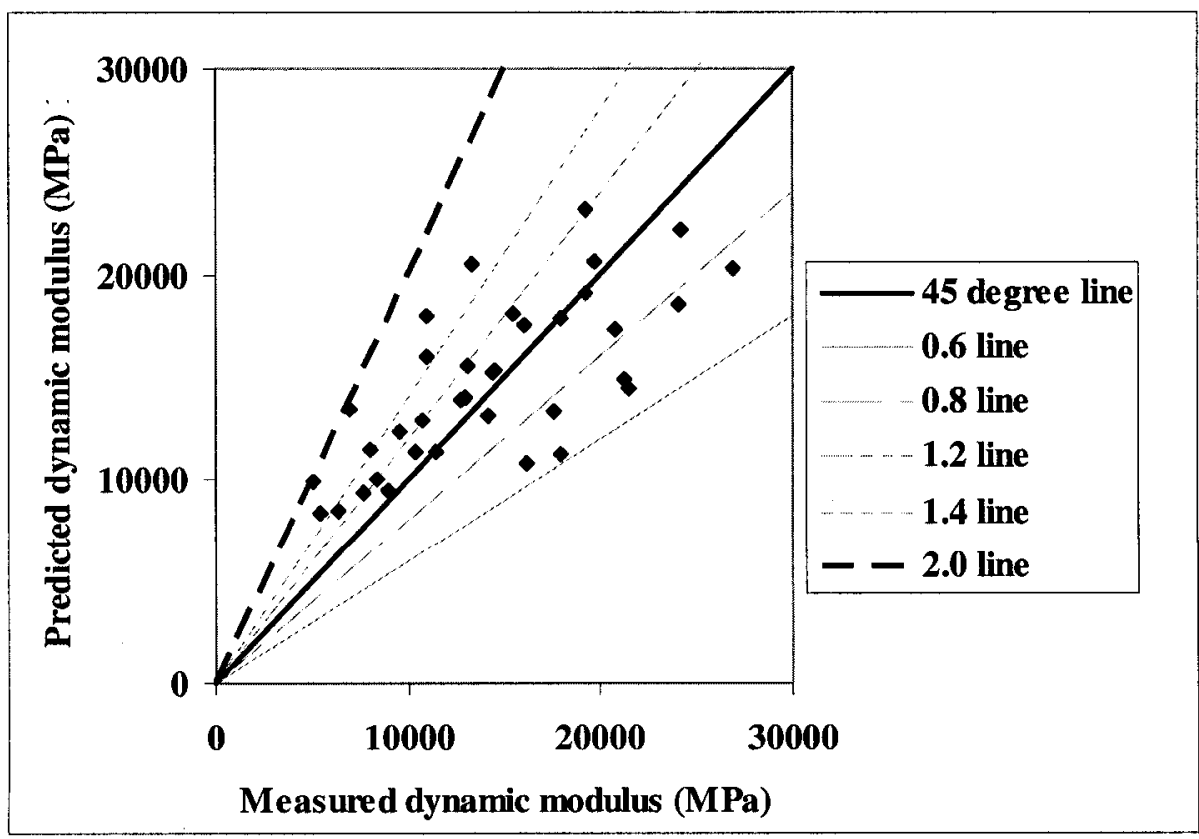

Figure 7.25. Dynamic modulus predictions at $-10^{\circ} \mathrm{C}$ using 2000 equation 


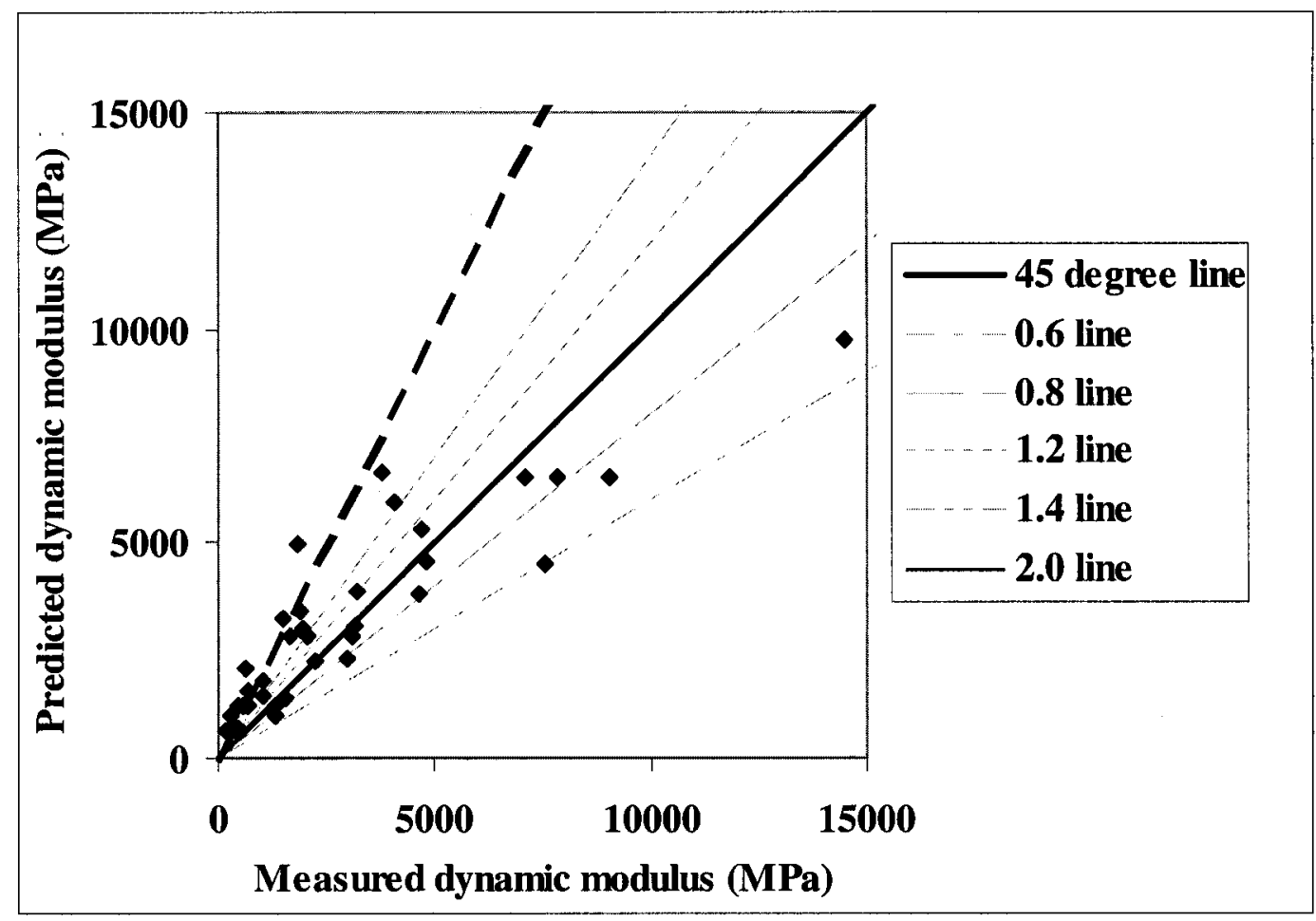

Figure 7.26. Dynamic modulus predictions at $+20^{\circ} \mathrm{C}$ using 1996 equation

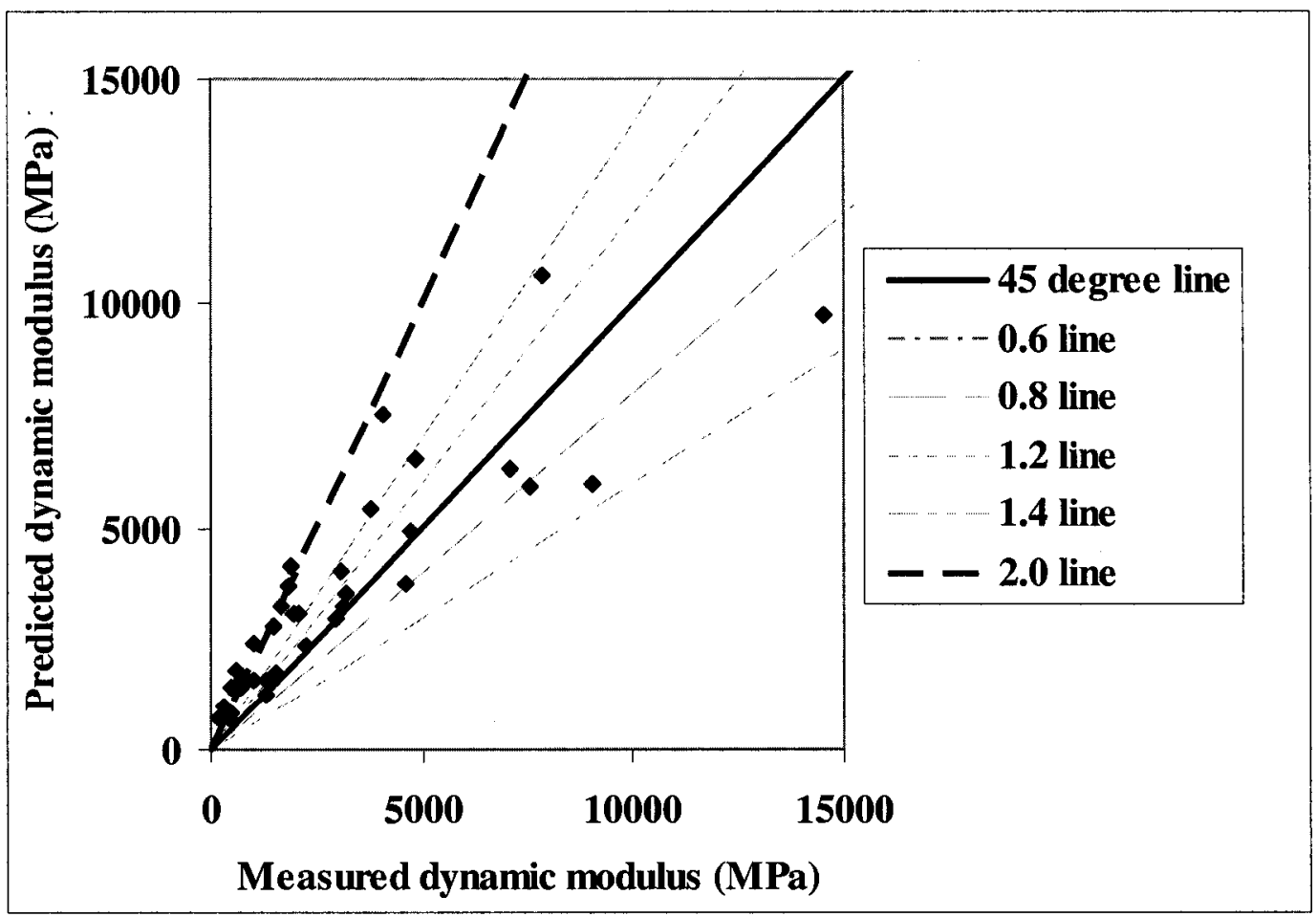

Figure 7.27. Dynamic modulus predictions at $+20^{\circ} \mathrm{C}$ using 2000 equation 
At a high temperature of $40^{\circ} \mathrm{C}$, over-estimation associated with the predictions made using the 1996 equation increased (deviated even further above the $45^{\circ}$ line) as can be seen in Figure 7.28. The majority of the data fall beyond the $40 \%$ over-estimation line (1.4 line). Over-predictions by the equation go beyond the $100 \%$ line ( 2.0 line), which is typical of a prediction with a modulus state represented by values lower than $500 \mathrm{MPa}$. The effectiveness of the performed predictions is clearly influenced by the temperature. This effect can be summarized as a clear shift from under-estimation to over-estimation as the temperature increases from cold to warm. This conclusion also applies to the 2000 predictive equation (see data plotted in Figure 7.29). However, a slight improvement was observed in the predictions made when a low dynamic modulus state prevails (less than $500 \mathrm{MPa})$.

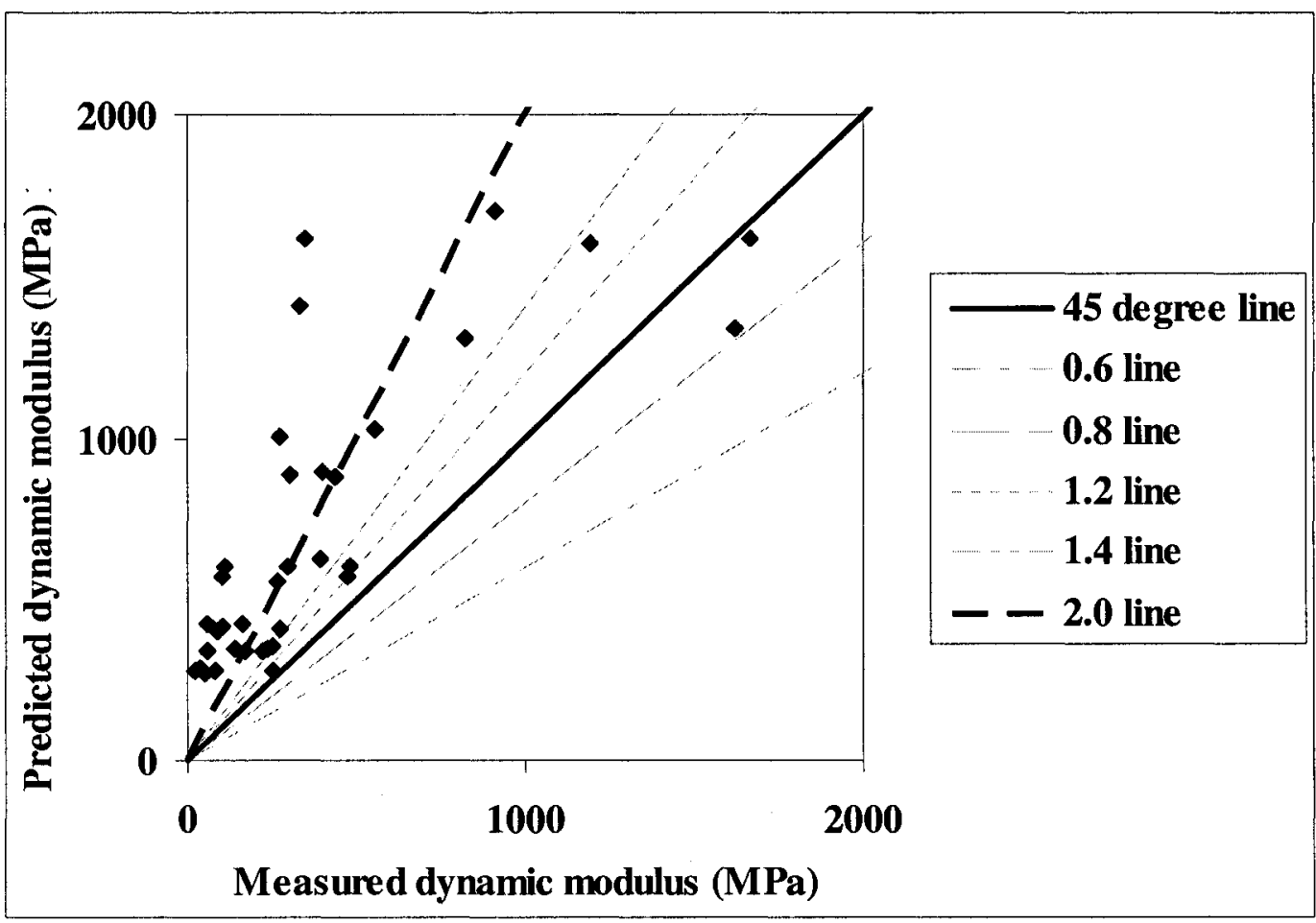

Figure 7.28. Dynamic modulus predictions at $+40^{\circ} \mathrm{C}$ using 1996 equation 


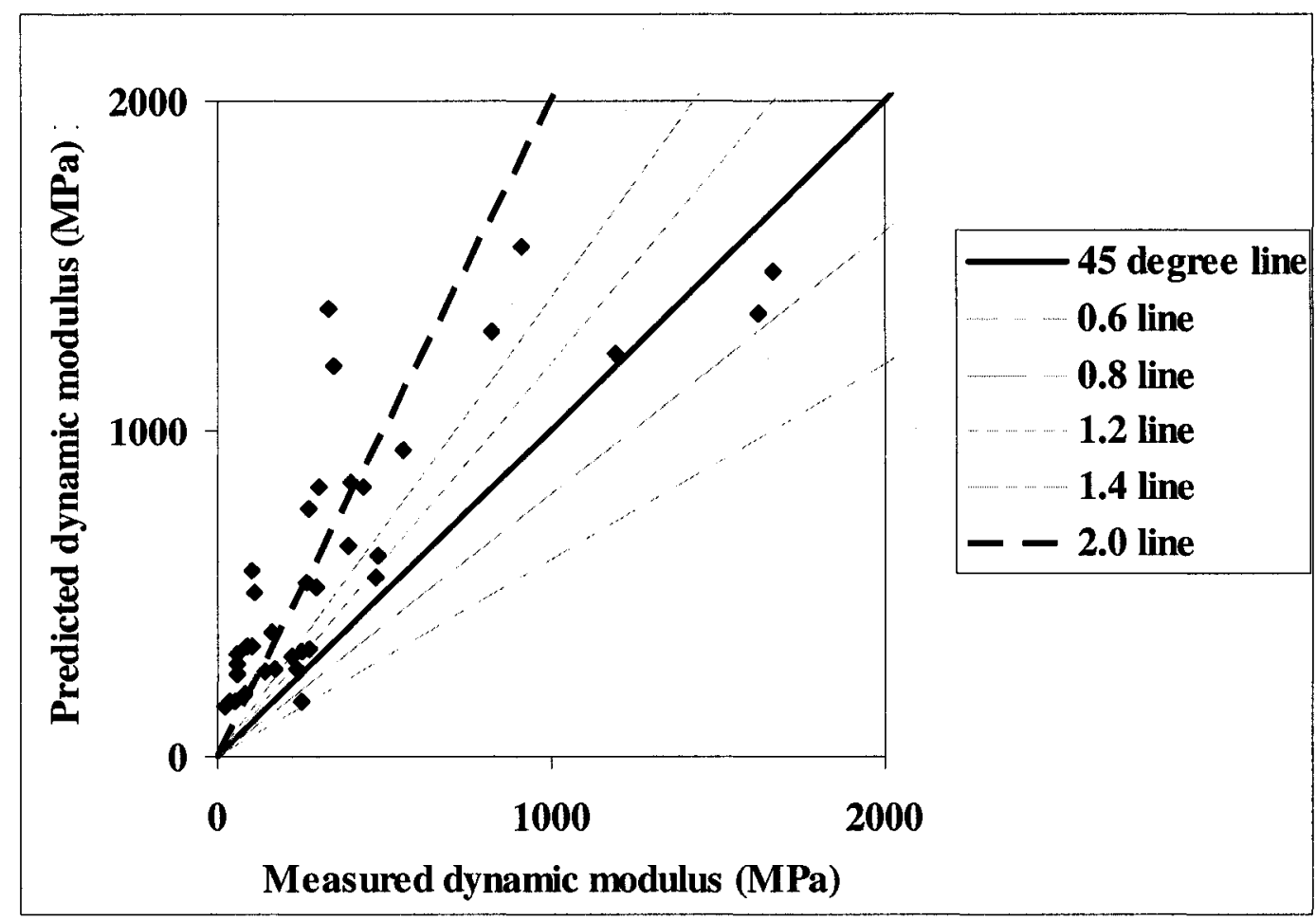

Figure 7.29. Dynamic modulus predictions at $+40^{\circ} \mathrm{C}$ using 2000 equation

The effect of temperature on the accuracy of predictions made using the 1996 and 2000 equations was also evaluated using statistical analysis. The mean absolute error as well as the average percent error was calculated for the two predictive equations at three temperatures including $-10,+20$, and $+40^{\circ} \mathrm{C}$. The results are presented in Table 7.7 . It is clear that the 2000 equation resulted in an improvement over capabilities of the 1996 equation especially at temperatures lying at the extremes of the selected range $(-10$ and $40^{\circ} \mathrm{C}$ ). The mean absolute error dropped significantly at these temperatures. The improvement brought by the 2000 equation is also clear when the average percent error is considered. At high and low temperatures, the average percent error dropped by as much as $30 \%$. Comparison of average percent error pertaining to the three temperatures revealed better predictive capabilities of the two equations at low temperature $\left(-10^{\circ} \mathrm{C}\right)$ compared to predictions made at medium $\left(20^{\circ} \mathrm{C}\right)$ and high temperatures $\left(40^{\circ} \mathrm{C}\right)$. 
Table 7.7. Results of statistical analysis related to temperature effect

\begin{tabular}{|c|c|c|c|c|c|c|}
\hline \multirow{3}{*}{ Equation } & \multicolumn{3}{|c|}{ Mean Absolute Error (MPa) } & \multicolumn{3}{c|}{ Average Percent Error (\%) } \\
\cline { 2 - 7 } & \multicolumn{3}{|c|}{ Temperature $\left({ }^{\circ} \mathrm{C}\right)$} & \multicolumn{3}{c|}{ Temperature $\left({ }^{\circ} \mathrm{C}\right)$} \\
\cline { 2 - 7 } & -10 & 20 & 40 & -10 & 20 & 40 \\
\hline 1996 & 6242 & 1039 & 329 & 38 & 66 & 208 \\
\hline 2000 & 3333 & 1116 & 256 & 26 & 75 & 146 \\
\hline
\end{tabular}

\subsubsection{Frequency Effect}

The effect of frequency on the accuracy of dynamic modulus predictions performed using the two equations was assessed by comparing predicted and measured values, considering all mix types and test temperatures. The results reflecting the impact of loading frequency are shown in Figures 7.30 to 7.35. A consistent trend was observed in all curves that represent predictions by the 1996 equation. At low moduli, the 1996 equation overpredicted dynamic modulus values. The equation has under-predicted dynamic modulus values within the large modulus state. However, the equation predicted values within the medium modulus state with deviations ranging from 20 to $30 \%$. At a loading frequency of $0.1 \mathrm{~Hz}$ (see Figure 7.30), dynamic modulus values were over-predicted within the low modulus state, which ranged from 0 to $1000 \mathrm{MPa}$. This range widened to $0-2000 \mathrm{MPa}$ for $1 \mathrm{~Hz}$ (Figure 7.32) and $0-5000 \mathrm{MPa}$ for $20 \mathrm{~Hz}$ (Figure 7.35). The range of dynamic modulus over which the equation under-estimated the measured values was also affected by the loading frequency. At a low frequency of $0.1 \mathrm{~Hz}$, the threshold beyond which the dynamic modulus values were under-estimated was approximately $7500 \mathrm{MPa}$. This threshold moves to higher values (10000 and $14000 \mathrm{MPa}$ at 1 and $20 \mathrm{~Hz}$ respectively).

Estimates of the dynamic modulus predicted using the 2000 equation are shown in Figures $7.31,7.33$ and 7.35 for loading frequency of $0.1,1$ and $20 \mathrm{~Hz}$ respectively. Overestimation by the 1996 equation within the low modulus state remained when the 2000 
equation was used. Furthermore, the range for which dynamic modulus values were overpredicted within the low modulus state increased with an increase in frequency and coincided with the range observed in the case of the 1996 equation $(0-1000 \mathrm{MPa}$ for 0.1 $\mathrm{Hz}, 0-2000 \mathrm{MPa}$ for $1 \mathrm{~Hz}$ and $0-5000 \mathrm{MPa}$ for $20 \mathrm{~Hz}$ ). The estimates made within the medium dynamic modulus state using the 2000 equation become less reliable in the case of 0.1 and $1 \mathrm{~Hz}$. Deviations of 20 to $30 \%$ observed with the 1996 equations increased to between 30 and $200 \%$. For a high frequency $(20 \mathrm{~Hz})$, the 2000 predictions within the medium dynamic modulus state reflected an improvement in terms of reducing the percentage of the deviation. However, the predictions switched from under-prediction to a blend of under- and over-prediction. The most noticeable improvement brought about by the 2000 equation is apparent for the high modulus state in which the early underestimates of $60 \%$ using the 1996 equation at the three levels of frequency became a mixed balance of over- and under-estimation, with a deviation of less than $30 \%$.

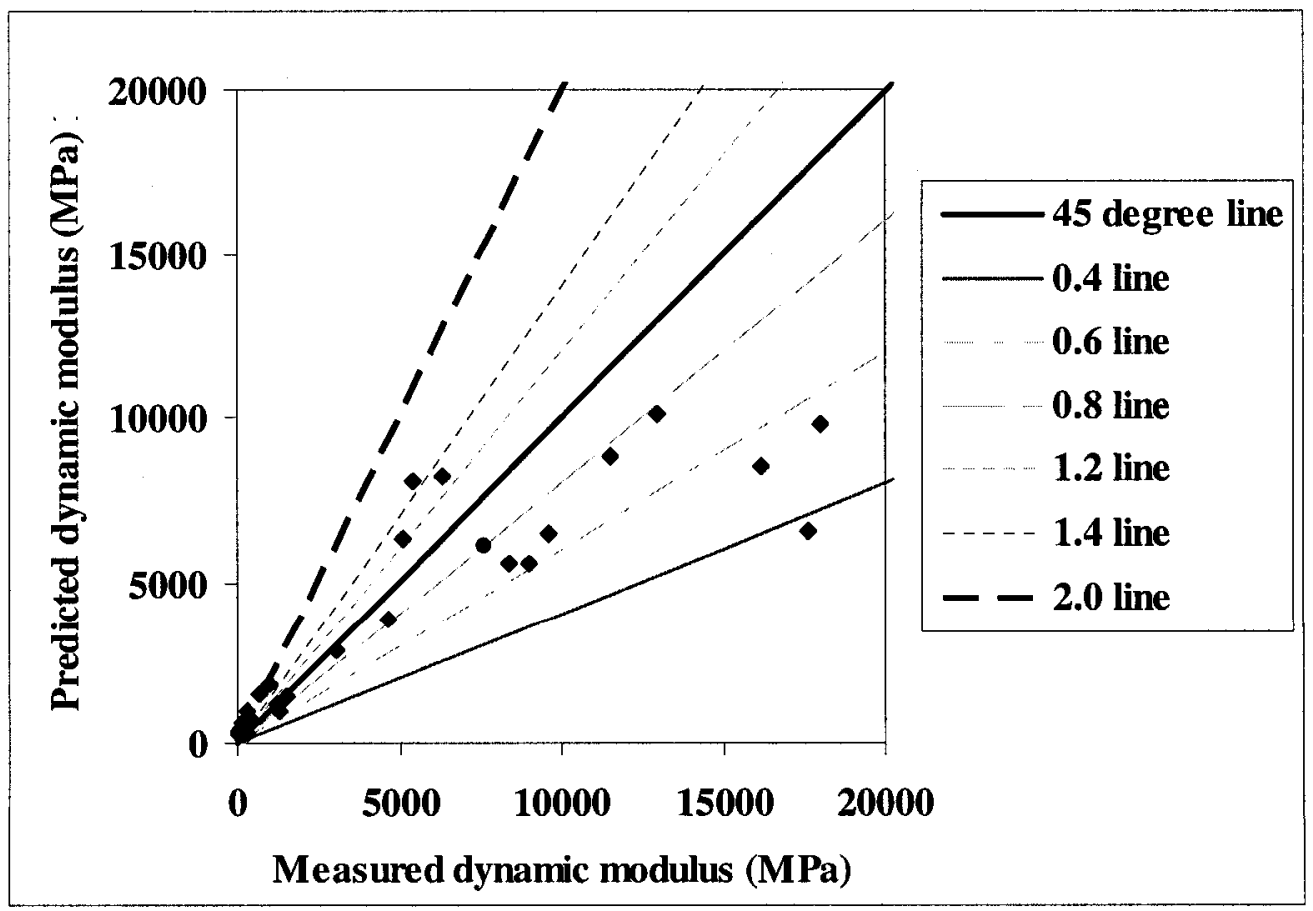

Figure 7.30. Dynamic modulus predictions at $0.1 \mathrm{~Hz}-1996$ equation 


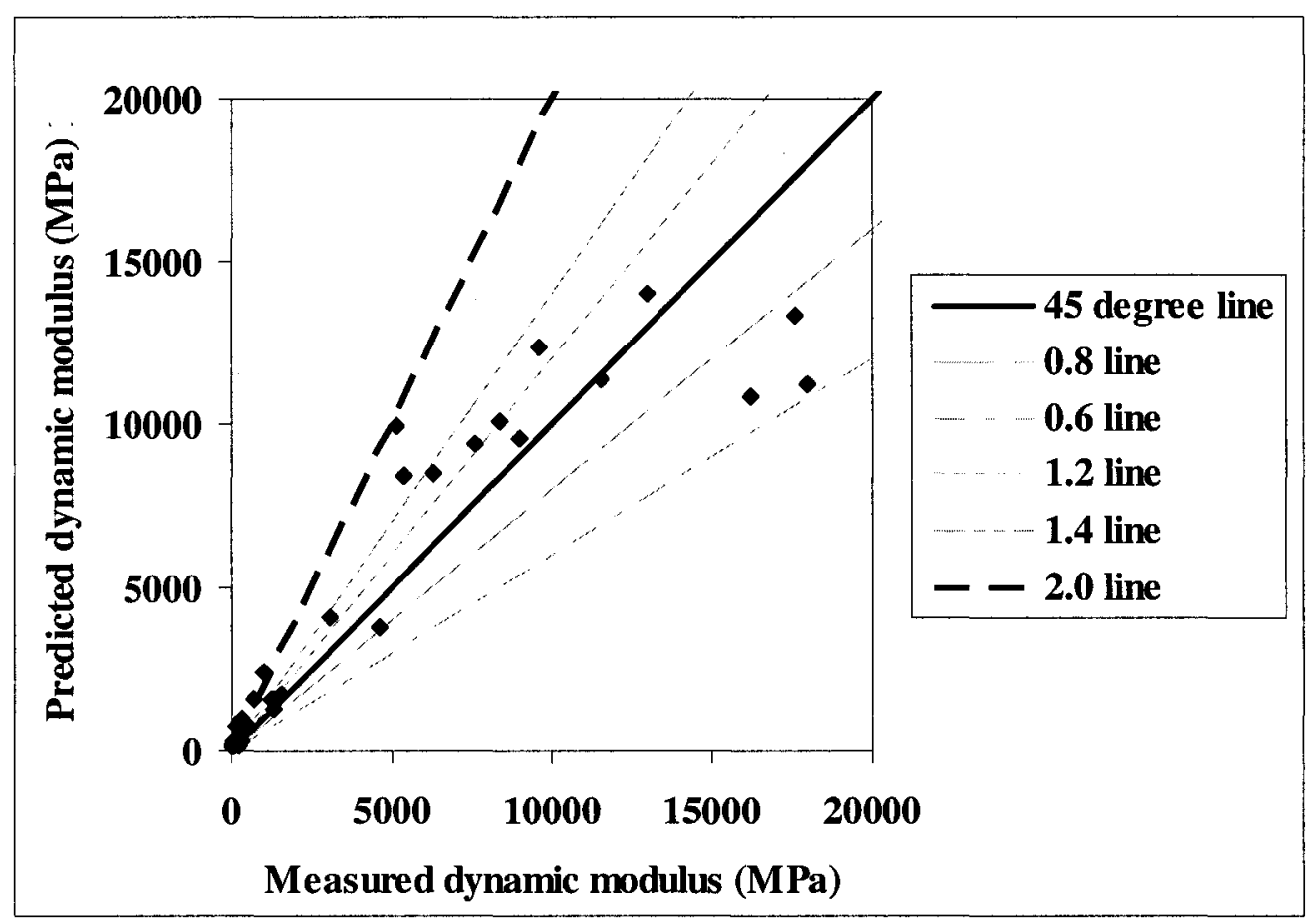

Figure 7.31. Dynamic modulus predictions at $0.1 \mathrm{~Hz}-2000$ equation

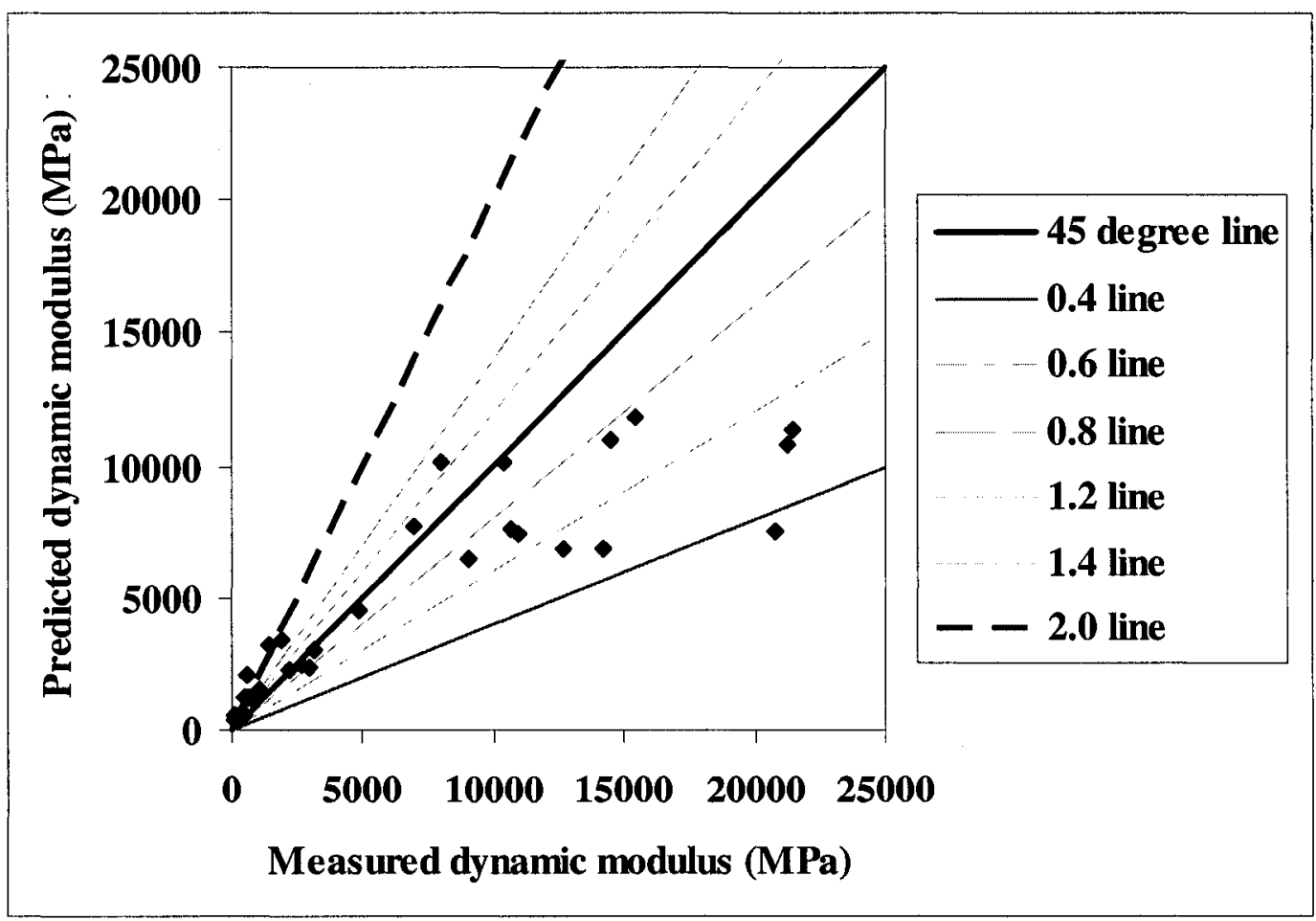

Figure 7.32. Dynamic modulus predictions at $1 \mathrm{~Hz}-1996$ equation 


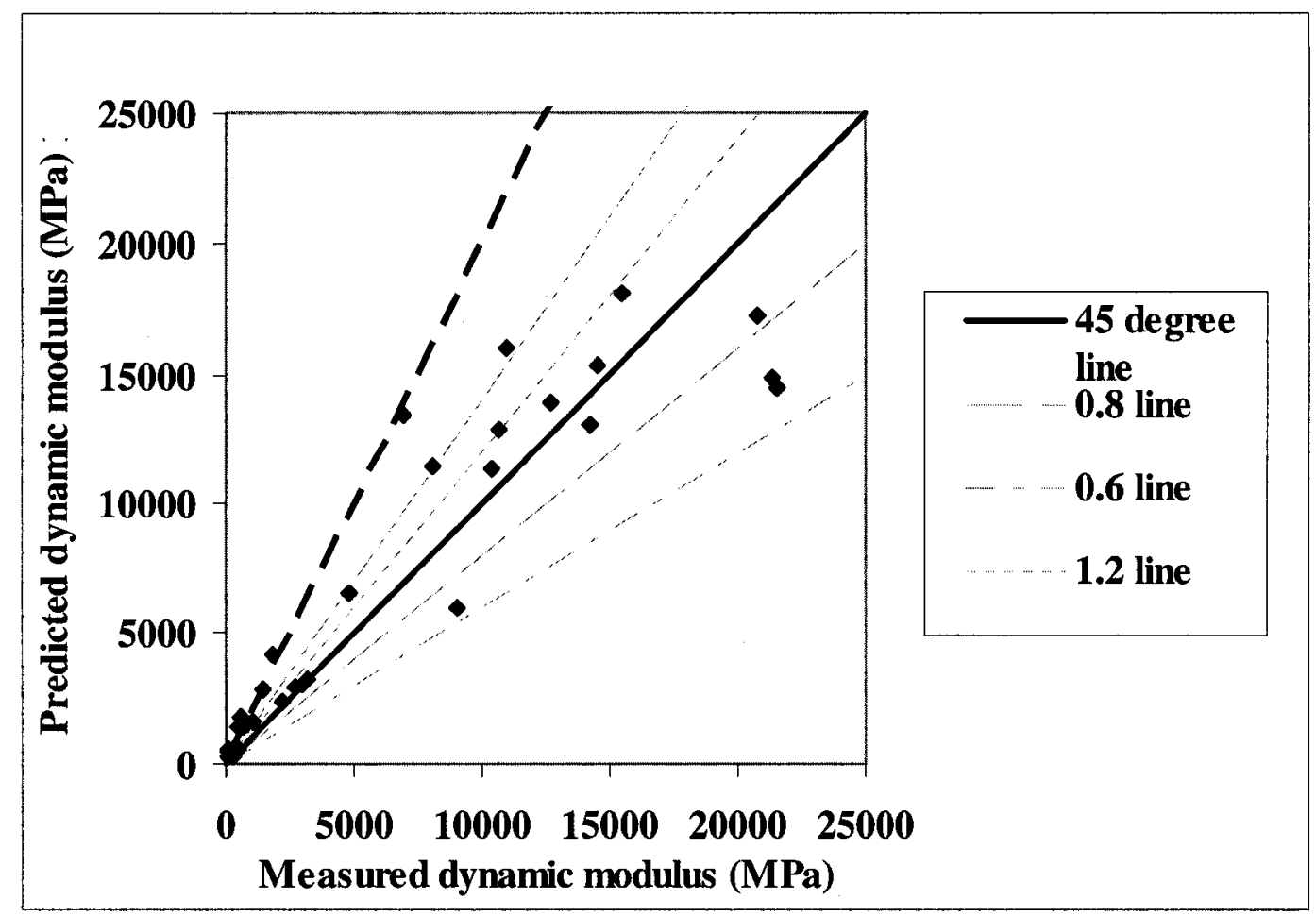

Figure 7.33. Dynamic modulus predictions at $1 \mathrm{~Hz}-2000$ equation

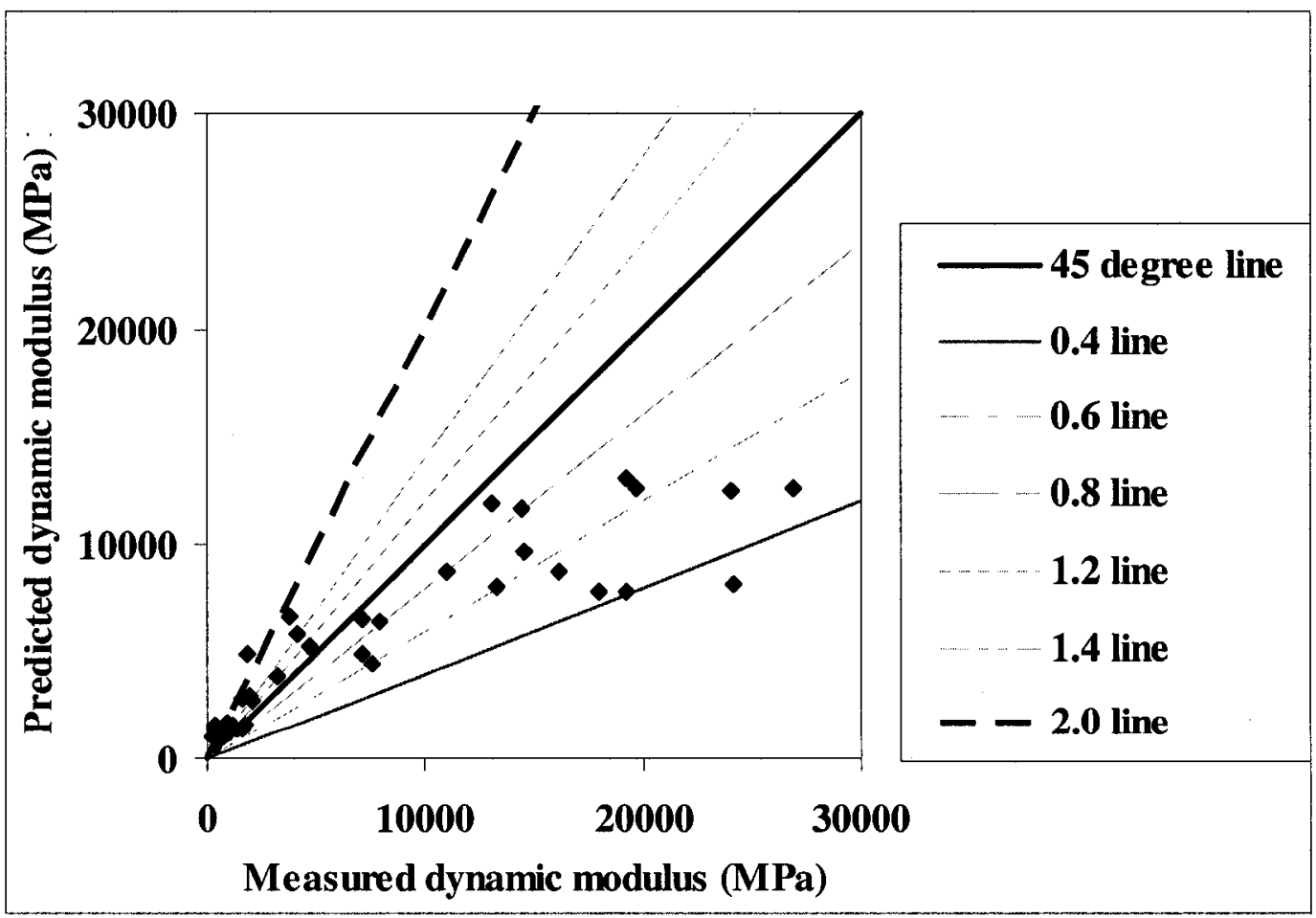

Figure 7.34. Dynamic modulus predictions at $20 \mathrm{~Hz}$ - 1996 equation 


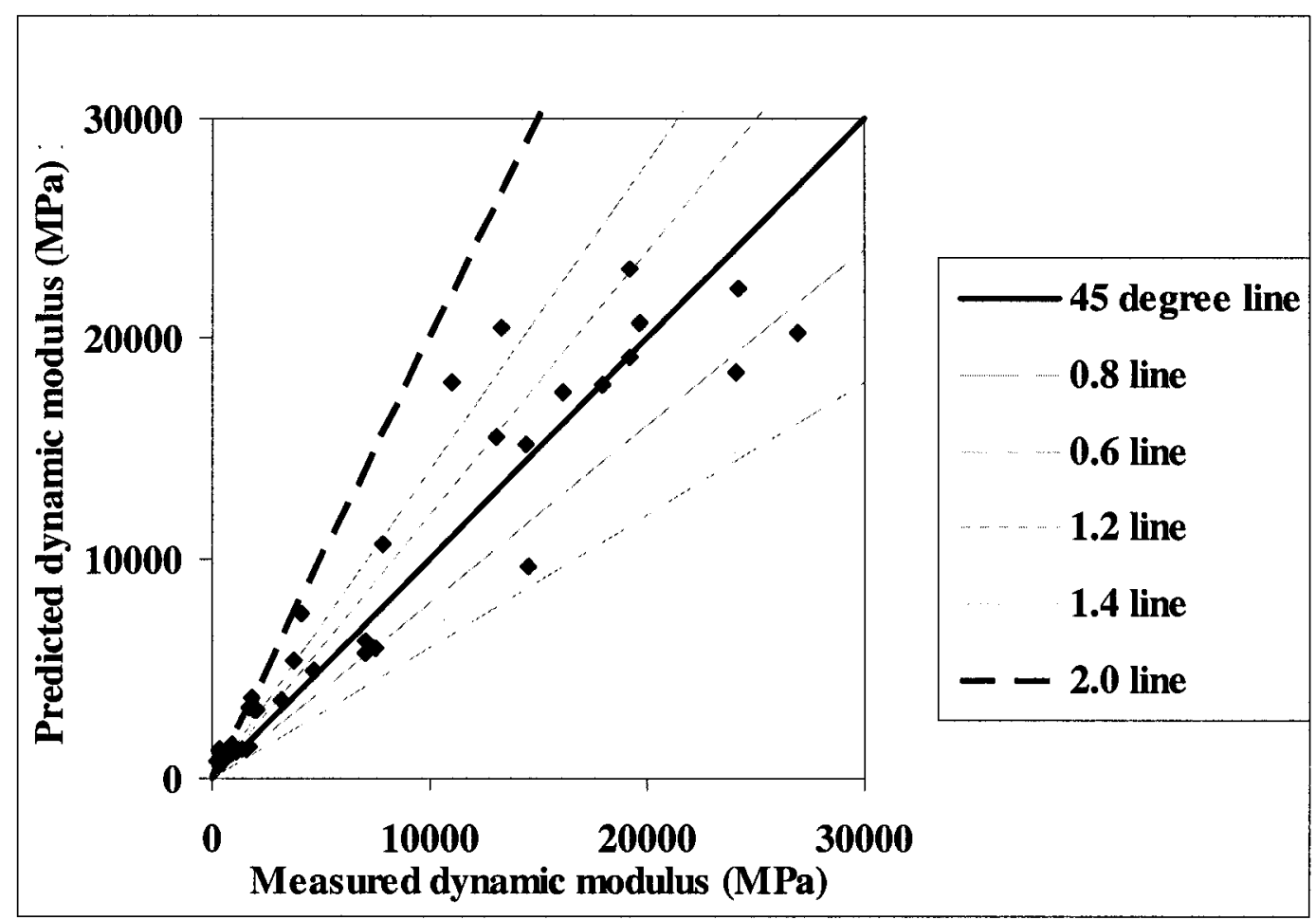

Figure 7.35. Dynamic modulus predictions at $20 \mathrm{~Hz}$ - 2000 equation

Similar to other effects, quantification of the effect of loading frequency on the accuracy of predictions made by the 1996 and 2000 equations was undertaken using statistical analysis. Table 7.8 shows that the mean absolute error and the average percent error incurred by using predictions of the two equations at different frequencies. The results reflect the relative superiority of the 2000 equation as the mean absolute errors at all frequencies are lower than those related to the 1996 equation. This improvement can also be seen in the drop of the average percent error when the 2000 equation was used instead of the 1996 equation. However this drop was less than $25 \%$ and the average percent error remained higher than $50 \%$ at all frequencies. Furthermore, the average percent error decreased with increasing frequency. 
Table 7.8. Statistical analysis results

\begin{tabular}{|c|c|c|c|c|c|c|}
\hline \multirow{3}{*}{ Equation } & \multicolumn{3}{|c|}{ Mean Absolute Error (MPa) } & \multicolumn{3}{c|}{ Average Percent Error (\%) } \\
\cline { 2 - 7 } & \multicolumn{3}{|c|}{ Frequency (Hz) } & \multicolumn{3}{c|}{ Frequency (Hz) } \\
\cline { 2 - 7 } & 0.1 & 1 & 20 & 0.1 & 1 & 20 \\
\hline 1996 & 1491 & 2069 & 3810 & 136 & 108 & 73 \\
\hline 2000 & 1136 & 1499 & 1967 & 103 & 94 & 56 \\
\hline
\end{tabular}

\subsubsection{Impact of Aggregate Gradation}

A comparison between predicted and measured dynamic modulus values was performed on the two mix design techniques adopted in this thesis. Figure 7.36 shows the results of the comparison performed between three Marshall-based mix designs (HMA 3, HMA 2 and HMA 1 mixtures). These mixes were prepared using a PG 52-34 binder. The dynamic modulus was predicted using the 1996 equation. The contrast between the predicted and measured values of the dynamic modulus reflects a common trend for these three mixes. Within the low modulus state, dynamic modulus values were over-predicted; within the high modulus state, these values were under-predicted. The HMA 3 mix, which was prepared using a fine-aggregate gradation, exhibited more deviation within the low modulus state compared with both the HMA 2 and the HMA 3 mixes prepared with a coarser aggregate gradation.

The same observation could be extended when comparing the HMA 2 and the HMA 1, with the HMA 2 gradation being finer than that of the HMA 1 mix. This trend was reversed for the high modulus state in which the deviations showed a higher underestimation of the modulus value for coarser mixes. Within this high modulus state, the deviation of the HMA 1 mix (40-60\%) was found to be greater than the deviation of the HMA 2 mix (20-40\%). Moreover, predictions of the HMA 3 mix behaviour are better than those of the HMA 2 mix. The observations formulated above for the PG 52-34 
binder were found to hold for other performance grades as seen in Figure 7.37, which shows the results for the PG 64-34 binder.

The predictions using the 2000 equation of the Marshall mix design for PG 52-34 is shown in Figure 7.38. The results clearly show that the predictions within the low modulus state did not improve. However, predictions for the high modulus state showed a noticeable improvement, with predictions that were only $40 \%$ lower than those for the test results. For the 2000 equation, the overall observations related to predictions made by the 1996 equation are still valid. However, the 2000 predictions differed substantially when the results of the mix that combined the PG 64-34 binder and the HMA 2 aggregate gradation were used (see Figure 7.39). The deviation was greater for the HMA 2 than for the HMA 3 mix.

The trend seen in mixtures prepared according to the Marshall mix design, using different performance-grade binders related to the effect of aggregate gradation, was examined for SuperPave mixes (See Figure 7.40). The 1996 predictions of the SP 2 mix were closer to the 45-degree line than those of the SP 1 mix especially within the high modulus state, thus confirming the trend seen in the Marshall mixes. This trend can be qualitatively summarized by the following statement: "The finer the gradation of the mix, the higher the deviation from actual test results within the low modulus state." Finer mixes showed better predictions for the high modulus state. This trend was reversed when predictions were made using the 2000 equation for the SuperPave mix prepared with PG 52-34 as shown in Figure 7.41. 


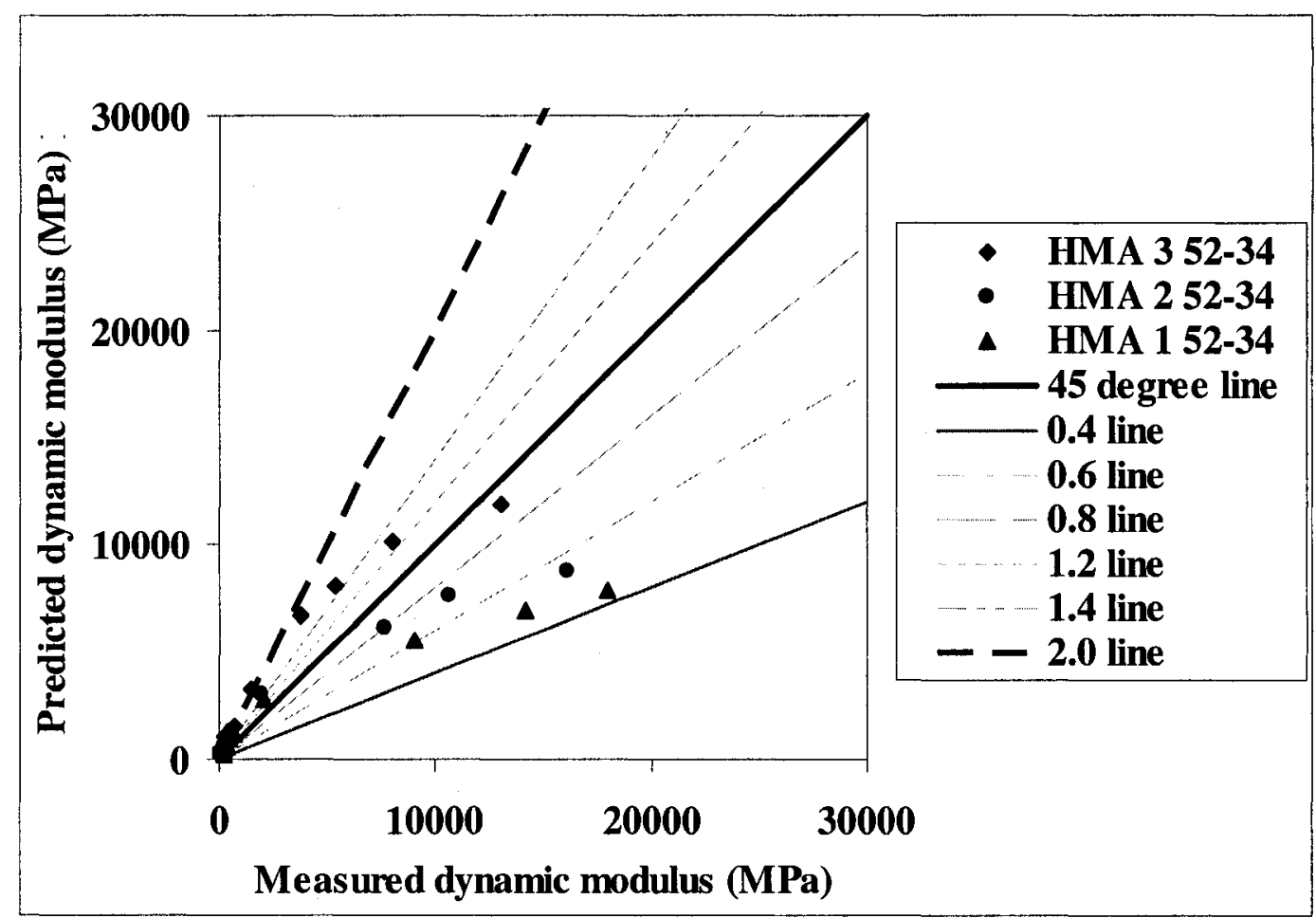

Figure 7.36. Aggregate gradation effect - 1996 equation

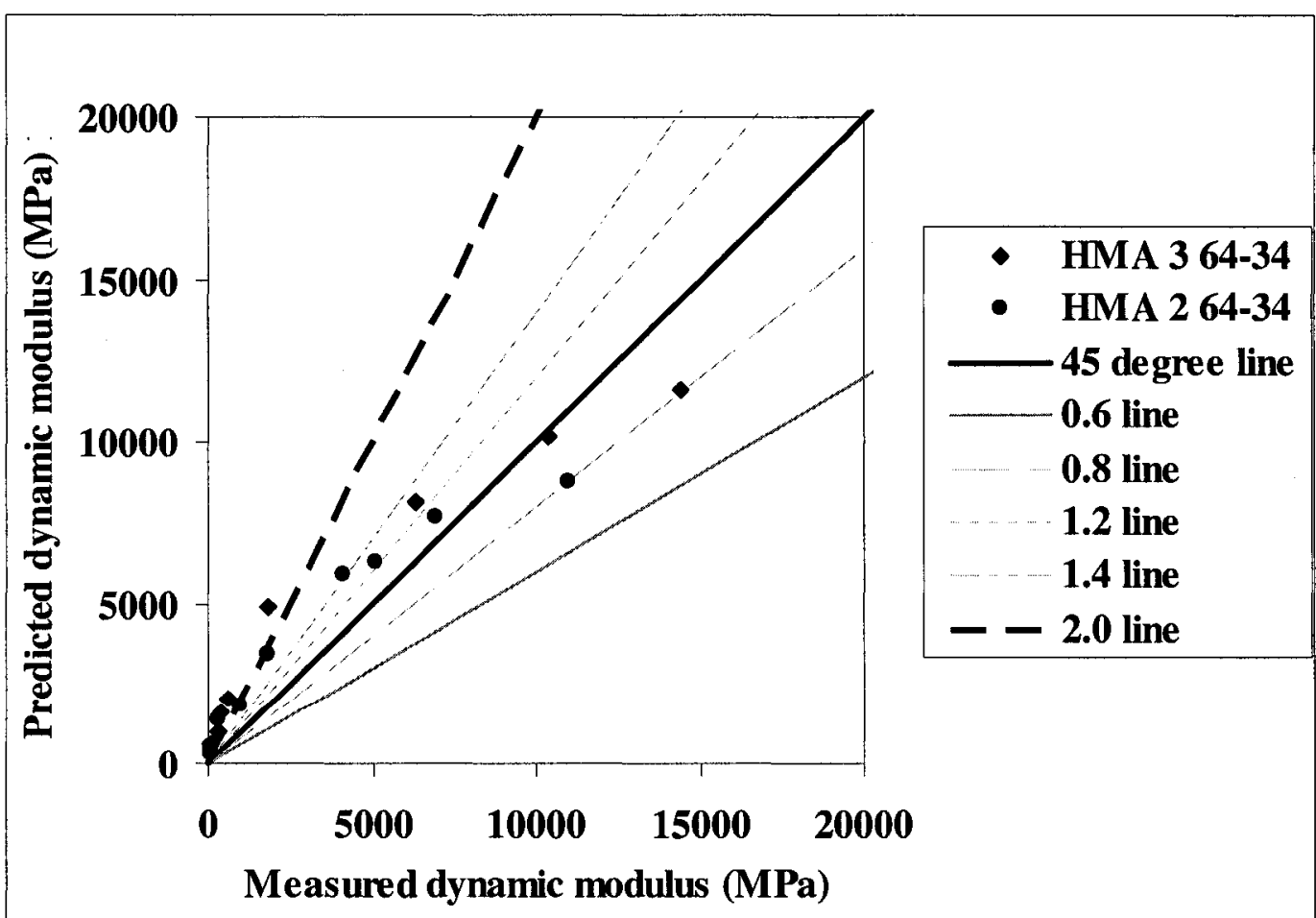

Figure 7.37. Aggregate gradation effect - 1996 equation 


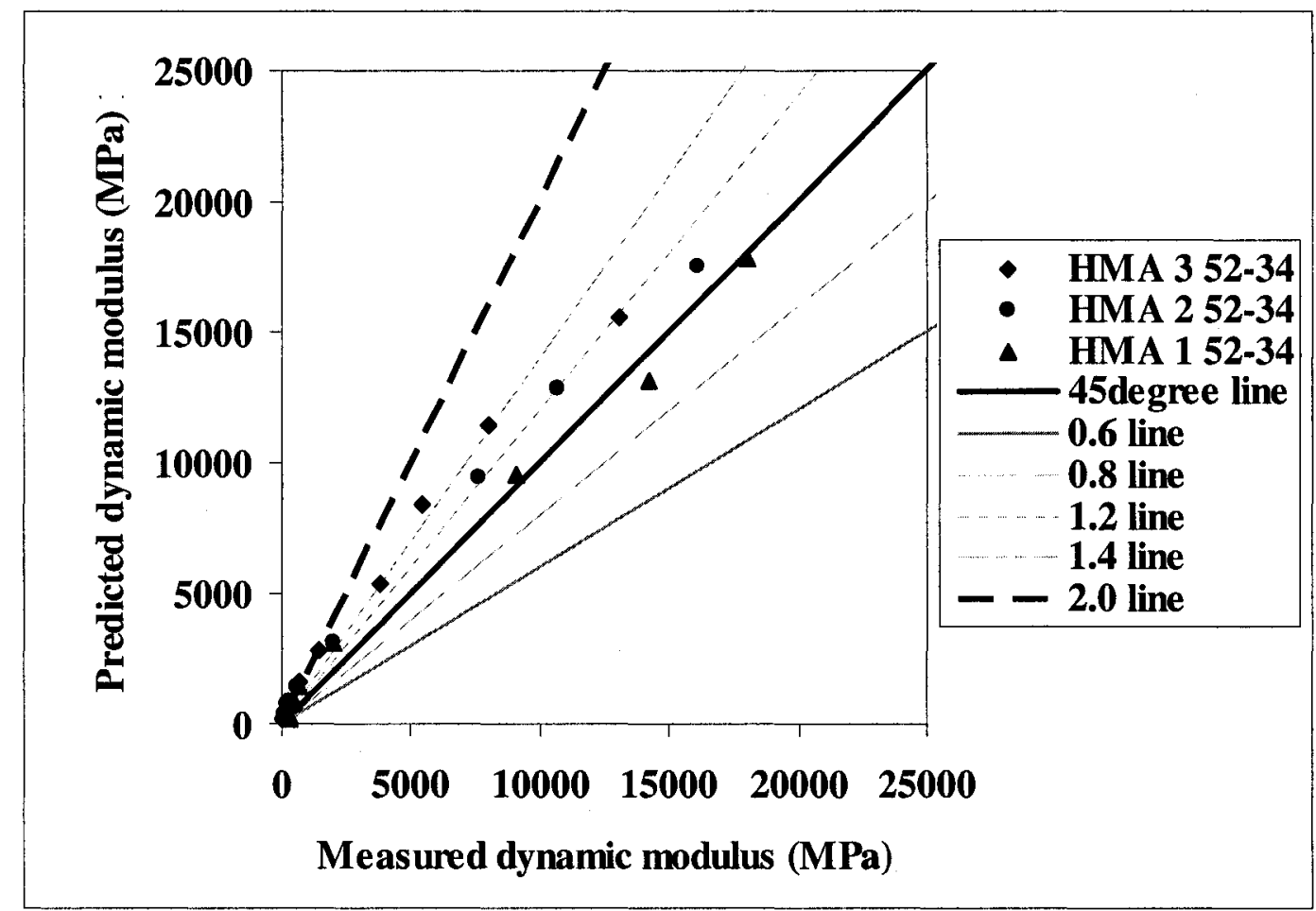

Figure 7.38. Aggregate gradation effect -2000 equation

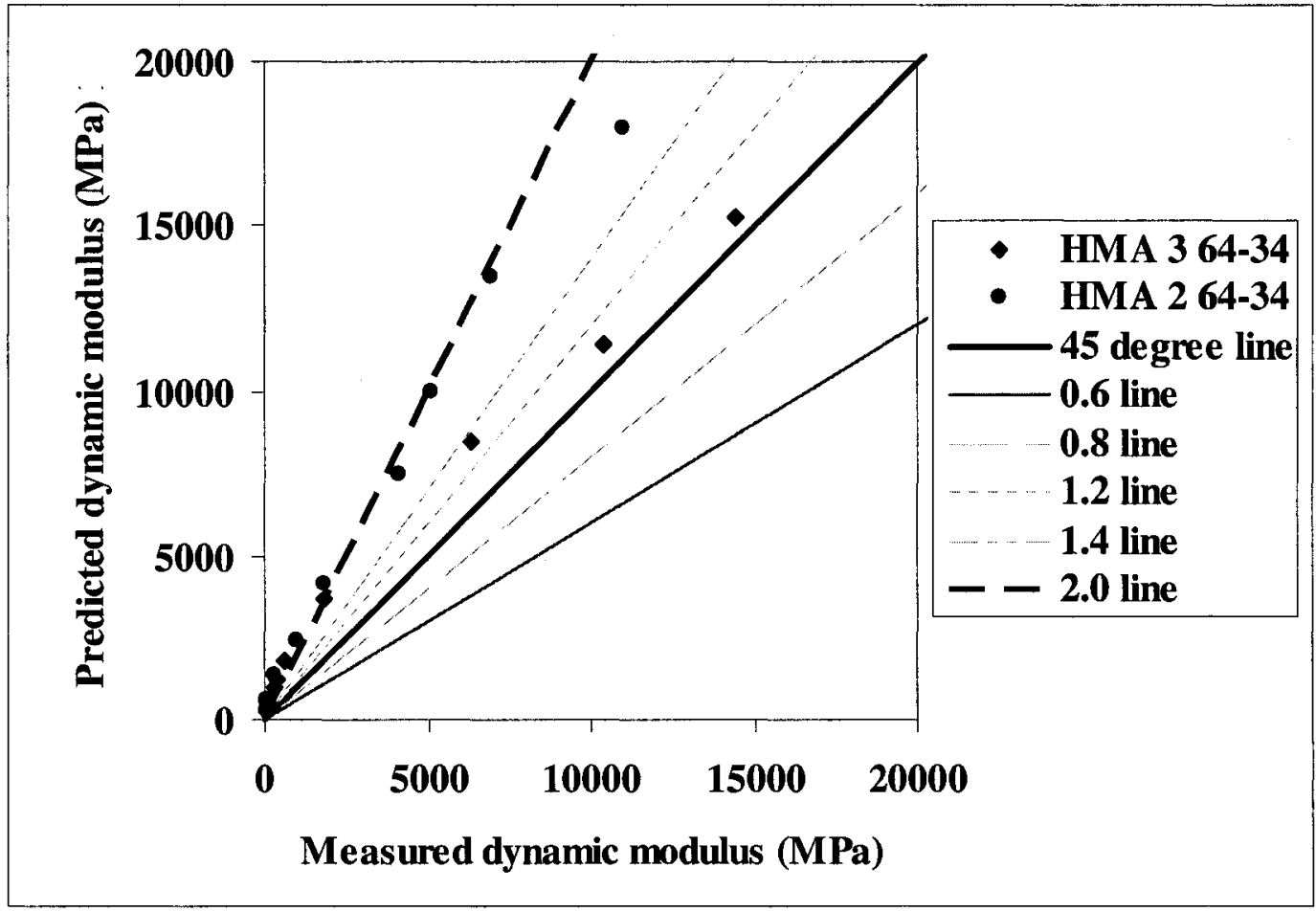

Figure 7.39. Aggregate gradation effect - 2000 equation 


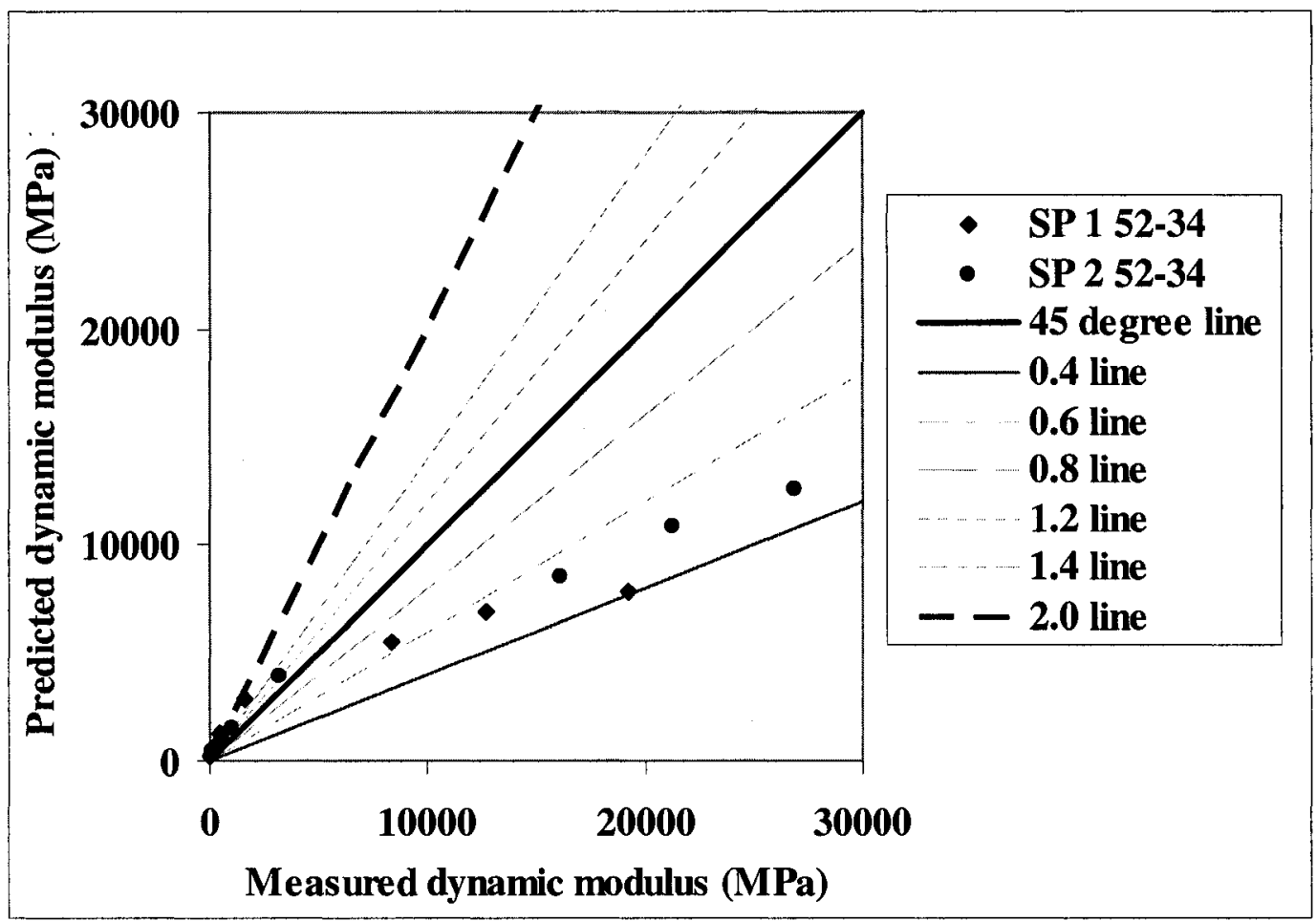

Figure 7.40. Aggregate gradation effect - 1996 equation

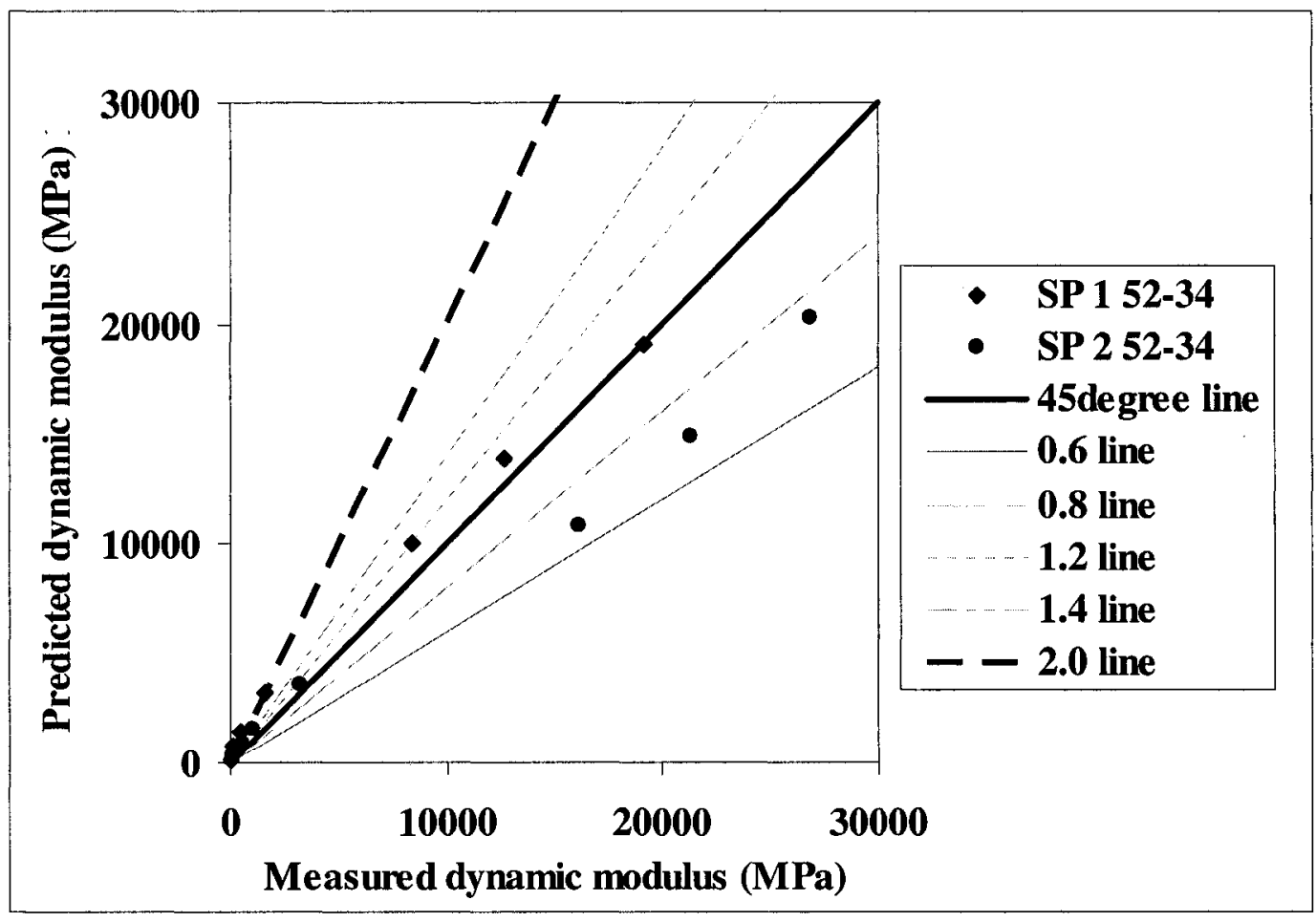

Figure 7.41. Aggregate gradation effect - 2000 equation 
To gain a more general understanding of the effect of gradation on the measured dynamic modulus values, the calculated mean average error and the average percent error for Marshall mixes with the PG 52-34 binder were summarized in Table 7.9. The mean absolute error increased with coarser mixes for the 1996 predictions. However, this trend reversed when the 2000 equation predictions were considered. The average percent error calculated for the two predictive equations showed a consistent trend where coarser mixes were better predicted than finer mixes.

Table 7.9. Results of statistical analysis for Marshall and SuperPave mixes

\begin{tabular}{|c|c|c|c|c|c|c|}
\hline \multirow{2}{*}{ Equation } & \multicolumn{3}{|c|}{ Mean Absolute Error (MPa) } & \multicolumn{3}{c|}{ Average Percent Error (\%) } \\
\cline { 2 - 7 } & HMA 1 & HMA 2 & HMA 3 & HMA 1 & HMA 2 & HMA 3 \\
\hline 1996 & 2559 & 1690 & 1423 & 53 & 159 & 255 \\
\hline 2000 & 491 & 963 & 1512 & 45 & 127 & 167 \\
\hline
\end{tabular}

\subsection{Summary}

The assessment of predictive equations performed in this study is critical for future efforts that pursue improvements in the ability to model the $\mathrm{AC}$ rheology. The establishment of these equations was a lengthy and tedious task. However, their accuracy is important for users of Level 3 design of the ASSHTO 2002 Guide. An assessment of the two predictive equations showed that the 2000 equation represents an improvement in accuracy compared with the older 1996 equation. Qualitatively, dynamic modulus predictions made with the 2000 equation improved accuracy within the high modulus state (dynamic modulus value greater than $15000 \mathrm{MPa}$ ) as shown in Figure 7.42 . However, the predictions made with the 2000 equation within the low modulus state (modulus lower than $15000 \mathrm{MPa}$ ) showed no improvements. Dynamic modulus values within the low range (lower than $4000 \mathrm{MPa}$ ) are similar in the two equations. Modulus predictions in the range of 4000 to $15000 \mathrm{MPa}$ switched from under-estimating the test 
results when the 1996 equation was used to over-estimating them when the 2000 equation was used.

Improvement in prediction accuracy in the 2000 equation over the 1996 equation was also apparent from the results of the statistical analysis performed across a number of factors expected to have impact on the dynamic modulus response. As can be seen in Table 7.10 the mean average error dropped by $38 \%$ and the average percent error by $14 \%$, when the 2000 predictive equation was used. However, the average percent error remained relatively high $(77 \%)$. Considering the elements investigated in this study, both predictive equations showed little or no sensitivity to the impact of the binder type on the mechanistic response. This limited sensitivity towards the nature of the binder also reflected in the high error determined when the equations were used to predict the modulus of finer mixes, which usually involves the use of high binder content. Also, the predictive equations showed limited sensitivity towards traffic speed (loading frequency).

Table 7.10. Overall evaluation of the 1996 and 2002 equations

\begin{tabular}{|c|c|c|}
\hline Equation & Mean absolute error (MPa) & Average percent Error (\%) \\
\hline 1996 & 2271 & 97 \\
\hline 2000 & 1415 & 77 \\
\hline
\end{tabular}




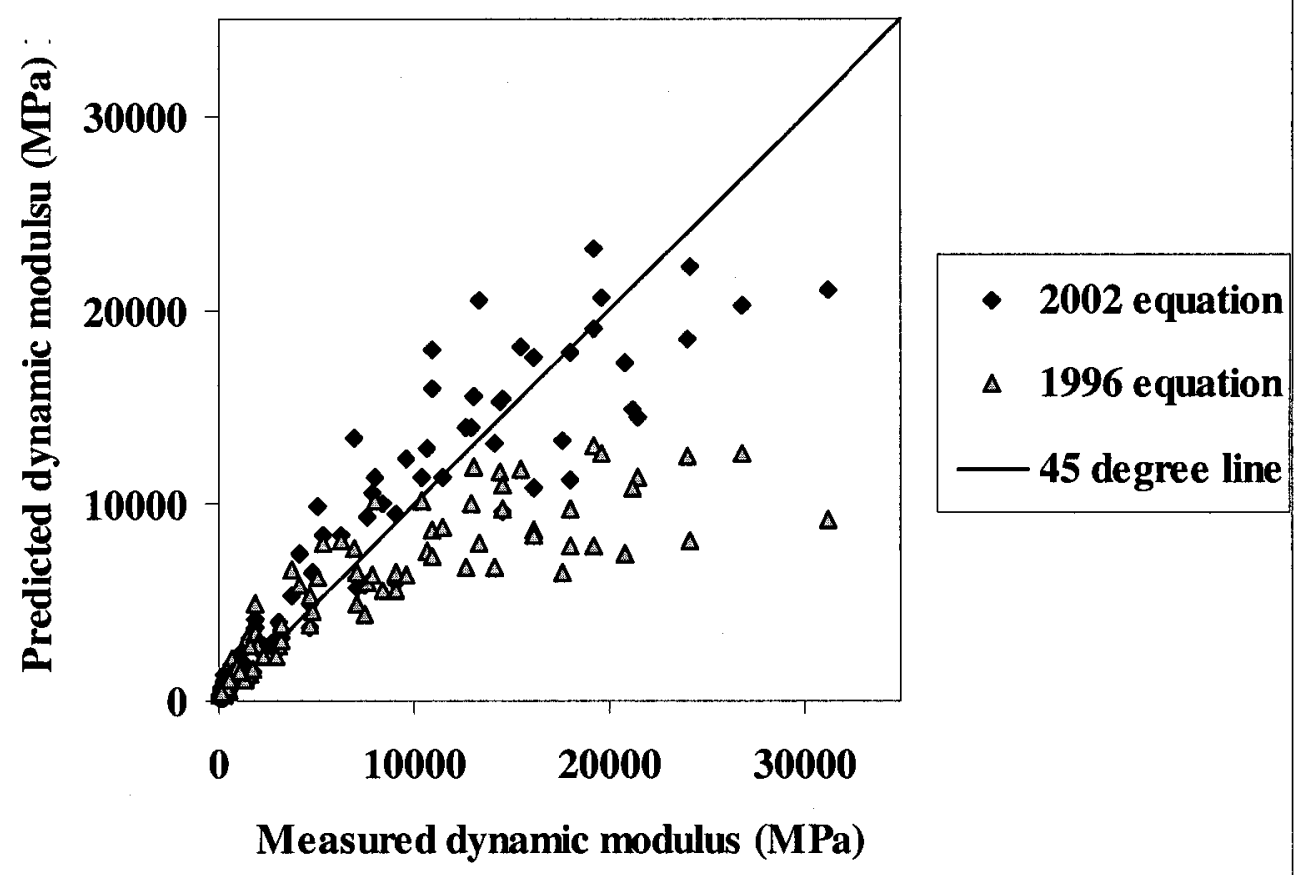

Figure 7.42 Comparison between predictions made with 1996 and 2000 equations 


\section{CHAPTER 8 \\ CONCLUSIONS AND RECOMMENDATIONS}

The main objectives of this thesis, which were related to the development of a mechanistic characterization technique for asphalt concrete, were met. The proposed mechanistic properties will facilitate roadway pavement design and analysis using a viscoelastic approach. These parameters could be used directly as input to recently developed analytical models or they could also be used to produce rheological models in the future to support more advanced practices. The following are conclusions and recommendations based on the outcome of the investigations performed in this thesis:

\subsection{Conclusions}

- A review of the literature revealed a gap in current pavement design and analysis practices; mainly, the absence of defined material properties that can effectively capture the mechanistic response of asphalt concrete materials. Current material characterization is limited to physical testing performed as part of the AC mix design task. This type of testing is not capable of supporting the desired level of performance-based design and analysis. In the absence of an adequate characterization technique, the road industry has relied on empirical pavement design procedures, mainly AASHTO design guides, originally based on road tests performed in the early 1960s. Material characterization has remained completely isolated from the requirements of the structural design process.

- Based on a review of experimental field data and the results of previous laboratory and analytical attempts to produce an effective rhelogical model for asphalt concrete, the complex modulus approach was selected and investigated in this thesis. The 
theoretical concept, based on a linear viscoelastic response, adequately approximates the behaviour of $\mathrm{AC}$ materials and produces the mechanistic characteristic responses necessary for effective pavement analysis.

- A laboratory test procedure was conceptualized taking into consideration the state of material and exposure conditions in roadway pavements. Tests were performed using the developed set-up and necessary refinements for accurately capturing stress and strains, and the phase lag between them, were made. The refinements were intended to make the test procedure sensitive to the impact of elements known to influence the AC response such as the state of the material, traffic and environmental conditions. Accordingly, a test protocol was developed and applied successfully to determine the complex modulus parameters including the dynamic modulus and the phase lag associated with the AC viscous behaviour. The characterization technique was found to be sensitive to known responses of $\mathrm{AC}$ materials subjected to different exposure conditions in the field including traffic variables (vehicle characteristics and traffic speed), temperature variations (cold versus warm). The characterization techniques also proved sensitive to the mix type as influenced by the properties of its components (aggregate and binder). A data-acquisition system was developed to facilitate automation of the process, which involved the recording of the test history and the collection of results in real time. Also, a data-reduction procedure was developed to process raw data and to prepare the results in a number of different forms that satisfy the requirements of a variety of analytical techniques.

- The applicability of the adopted characterization technique in performing pavement design and analysis using the ASSHTO 2002 Design Guide and the Huet-Sayegh 
model was examined successfully. Although these models are not yet validated, the analysis reflected the ability of these models, using the AC characteristics developed in this thesis, to produce results comparable with known performance trends.

- The refined test protocol was adopted in testing a wide range of asphalt concrete mixes compatible with the two most commonly used mix design techniquesMarshall and SuperPave. The data were processed and analyzed, with the results reflecting the sensitivity of the developed approach to known behavioural patterns and the performance of engineered materials. To encourage the application of the developed characterization technique in running the 2002 Design Guide and other relevant rheological models, a database, the "Material Library," was developed and populated with the data from the thesis test results. Other users are invited to participate in the Material Library by further "populating" it with a wider range of mix types and exposure conditions.

- The developed database was used to directly evaluate the validity of the rheological model adopted in Level 3 of the ASSHTO 2002 Design Guide. This simplified design level is intended to circumvent the need to conduct the mechanical test to obtain the properties required to run the full version of the finite element model in Level 1. The evaluation covered two of the most common dynamic modulus predictive equations-one older equation and a new one presumed to be an improvement on the older one. The evaluation process revealed that the implemented 2000 predictive equation slightly improved overall accuracy. However, deviations of the predicted dynamic modulus values from the measured ones remained high, and some factors 
that influence accuracy, which were determined with laboratory test data, were identified:

- Although the two equations successfully discriminated between the two uniquely different binder grades by rating them correctly with respect to each other, both equations failed to quantify the dynamic modulus as measured in the laboratory at low temperatures.

- A temperature change from cold to warm resulted in a switch from under- to overprediction of the dynamic modulus when compared with the test results. The switch proved to be more pronounced in the case of the 1996 equation than in that of the 2002 equation.

- In the 1996 equation, increasing the magnitude of loading frequency widens the range over which the equation will over-predict the dynamic modulus within the low modulus state and increases the threshold beyond which the dynamic modulus is under-estimated.

- Employing either of the equations to predict the modulus of mixes prepared with finer aggregates decreases the accuracy of the predictions within the low modulus state and improves it within the high modulus state. 


\subsection{Recommendations}

- Attempts were made during the laboratory investigation to use two extensometers simultaneously to control the test. Unfortunately, this objective was not achieved because of the limited capabilities of the adopted MTS system. The extensometer not used as a control mode, was unable to accurately record the magnitude of the achieved displacements. Accordingly, readings from this extensometer were ignored and only displacements recorded by the other extensometer were considered. The accuracy of the recorded displacement amplitudes became a critical factor, and it was the reason behind frequent assessment of recorded strain used in this study. It is important that future investigations of the complex modulus test obtain dual measurements using two independent extensometers.

- The fact that a strain-controlled loading mode was used in this study means that the data must be compared with that from tests performed using the stress-controlled mode. The use of the stress-controlled mode was justified in the literature as being easier. Experience gained in this study suggests that the strain-controlled mode is also practical. More importantly, using the strain-controlled loading mode helps guard against exceeding the strain values beyond which permanent deformation may take place, which could jeopardize the adopted linear viscoelastic response assumption. Future analytical development may facilitate the inclusion of a permanent deformation component in the analysis, in which case, the test capabilities should be extended to cover the requirements of such an analysis.

- Although a wide range of mix types that are commonly used as sub-layers of the AC layer were used in this study, these mixes are still within specific limits and within the 
selected classes. Different gradation limits within these traditional classes based on physical properties are expected to produce different mechanistic responses. Hence, further studies are needed to evaluate different mix types within each class and to reclassify them, if necessary, according to the determined mechanistic response. This recommendation is based on reports suggesting that the performance of some mixes was not consistent.

- The nature of the curing process in the $\mathrm{AC}$ mix is not well understood. Because of the complicated nature of this parameter, the evaluation of the curing effect in this thesis was not finalized. However, a sample testing age was selected to maintain a unified testing standard. A judgment should be made by the user based on local conditions related to the scheduling of the road opening to traffic after overlaying an existing road with a fresh layer of AC.

- The characterization of $\mathrm{AC}$ mixes in this thesis and the results used to populate the Material Library were achieved using samples prepared according to guidelines recommended by the majority of road authorities, and based on setting a specific density range (4 to $6 \%$ air voids). The requirements related to regionally mandated construction quality or to specific mix types, such as Stone Matrix Asphalt (SMA), may dictate a lower or higher percentage of air voids. In the field, lower air void contents were reported ( 8 to $11 \%$ ), which will have some impact on the $\mathrm{AC}$ mechanistic response as proven in this study and will also have a negative impact on performance. Construction specifications include penalties. The scale of such penalties is currently being debated by the industry. Until performance prediction can be made with analytical models, populating the material with mechanistic properties 
achieved with different percentages of air void contents may support efforts to establish a more acceptable weighing-scale for evaluating the quality achieved in a construction project. 


\section{APPENDIX A}

\section{COMPLEX MODULUS TEST PROTOCOL}

AND DATA REDUCTION PROCEDURE 


\section{Scope}

This document describes procedures for determining the complex modulus and phase angle of compacted asphalt concrete samples over a range of pavement temperatures and loading frequencies. Also included is a procedure for reducing complex modulus data. This procedure is applicable to laboratory-prepared specimens of mixtures with nominal maximum size aggregate less than or equal to $19 \mathrm{~mm}(0.75 \mathrm{in})$.

Disclaimer: This standard does not address safety issues and problems associated with its use. Establishing appropriate safety and health practices and determining the applicability of regulatory limitations rely solely on the user of this standard.

\section{References}

2.1 ASTM Standards:

D 3496 Method for Preparation of Bituminous Mixture Specimens for Complex Modulus Testing

口D3549 Standard Test Method for Thickness or Height of Compacted Bituminous

Paving Mixture Specimens

口D3515 Specification for Hot-Mixed, Hot-Laid Bituminous Paving Mixtures

口2041 Maximum Specific Gravity and Density of Bituminous Paving Mixtures

2.2 AASHTO Standards

口TP4 Standard Method for Preparing and Determining the Density of Hot Mix Asphalt

(HMA) Specimens by Means of the SHRP Gyratory Compactor

口PP2 Practice for Mixture Conditioning of Hot Mix Asphalt (HMA)

口T269 Percent Air Voids in Compacted Dense and Open Bituminous Paving Mixtures

口T209 Maximum Specific Gravity and Density of Bituminous Paving Mixtures

口T166 Bulk Specific Gravity of Bituminous Paving Mixtures Using Saturated Surface-

Dry Specimens

\section{Terminology}

3.1 Dynamic Modulus: The absolute value of the complex modulus that defines the elastic properties of a linear viscoelastic material subjected to a sinusoidal loading, $\left|E^{*}\right|$.

3.2 Complex Modulus: A complex number that defines the relationship between stress and strain for a linear viscoelastic material, $E^{*}$.

3.3 Storage Modulus: The real part of the complex modulus that represents the elastic contribution of the bituminous mixture behaviour $E_{l}$.

3.4 Loss Modulus: The imaginary part of the complex modulus that represents the viscous contribution of the bituminous mixture behaviour, $E_{2}$.

3.5 Linear Material: A material whose stress to strain ratio is independent of the loading stress applied.

3.6 Phase Angle: A value that shows time dependency of asphalt materials and defines the lag between applied strain and the resulting stress.

3.7 Linear Viscoelastic: Within the context of this test method, it refers to bituminous behaviour in which the complex modulus is independent of stress or strain amplitude.

\section{Summary of Test Procedure}

A strain-controlled test is adopted within this protocol to perform the complex modulus test. A sinusoidal axial displacement corresponding to $100 \mu \varepsilon$ is applied at given temperatures and loading frequencies. The resulting axial stresses are measured and used together with the applied strains to calculate the dynamic modulus, and the phase angle at various temperatures and loading conditions. 


\section{Significance}

5.1 The value of the dynamic modulus reflects the stiffness of the mixture and its behaviour at designated frequency and temperature.

5.2 Dynamic modulus values measured over a range of temperatures and frequencies of loading can be shifted into a master curve that can be utilized for performance analysis using analytical models.

5.3 the dynamic modulus values can be used to generate rheological parameters that can be used by models to predict $\mathrm{AC}$ materials performance.

\section{Apparatus:}

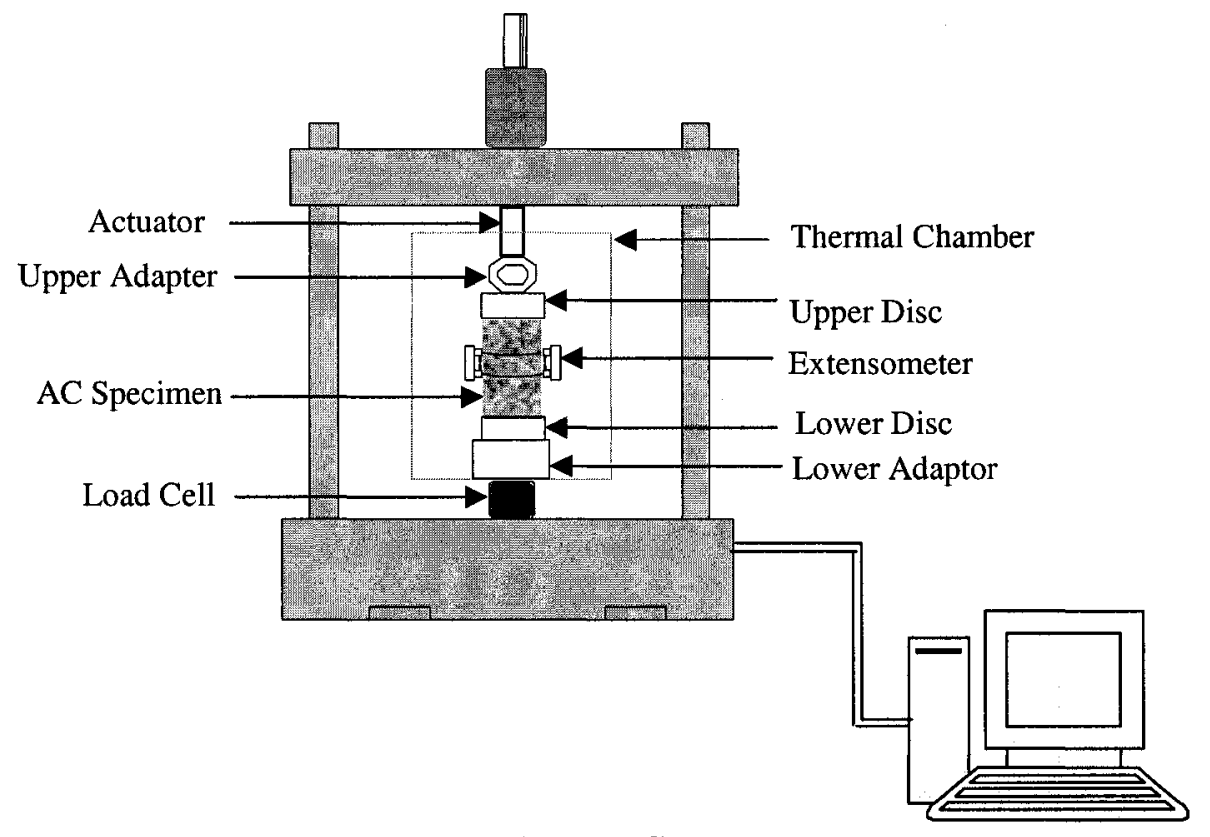

\section{Figure 1: Test Set-Up}

The Dynamic Modulus Test System consists of a hydraulic loading frame, environmental chamber, measuring systems, and a personal computer. See Figure 1.

6.1 Loading Frame: A servo-hydraulic loading frame rated at $100 \mathrm{kN}$ with an integrally mounted 5-inch stroke actuator should be used. The testing machine should be capable of applying an axial sinusoidal stress not less than $6.0 \mathrm{MPa}(800 \mathrm{psi})$ to produce a strain magnitude of $100 \mu \varepsilon$ over a range of frequencies from 0.1 to $20 \mathrm{~Hz}$ and temperatures from -10 to $+40^{\circ} \mathrm{C}$. The guidelines presented in this test protocol have been developed based on the use of the Material Testing System (MTS-TestStar IIs 810). Slight differences may be noticed if a different loading system is used.

6.2 Temperature-Control System: A chamber for controlling the specimen at the desired test temperature is required. The environmental chamber must be capable of controlling the temperature of the specimen over a temperature range from -10 to $40{ }^{\circ} \mathrm{C}$ to an accuracy of $\pm 0.5^{\circ} \mathrm{C}$. The thermal chamber should be large enough to accommodate the test specimen and other accessories such as measuring devices (strain gauges).

6.3 Controlling System: A built-in computer program MultiPurpose Testware (MPT) program, an MTS product, should be used to control the MTS system, perform the test according to the specifications developed in this thesis and to collect raw data. The programm should be capable of measuring and recording the time history of applied displacements and corresponding axial loads. 
6.4. Load cell: The applied load should be measured using an electronic load cell in contact with the bottom hardened steel disc through a steel adaptor. It was found that force measurements are more accurate when the load cell is not in contact with the moving actuator (See Figure 1). The capacity of the load measuring system should not be less than $45 \mathrm{kN}$ (10 kips).

6.5. Strain Measurements: Axial displacements should be measured using an extensometer capable of reading displacements corresponding to $100 \mu \varepsilon$. The strain measuring sensors should be mounted with springs on the side of the sample at midheight. It is recommended that a second sensor be placed $180^{\circ}$ apart from the first gauge if the MTS system used is capable of maintaining displacement control using two gauges (See figure 2).

6.6 Hardened Steel Discs: Hardened steel disks, with a diameter equal or greater than that of the test specimen are required on top and bottom of the specimen to transfer the load from the testing machine to the specimen (See Figure 2).

6.7 End Treatment: Friction reducing end treatments should be placed between the specimen ends and the hardened steel disks. The end treatments should consist of $0.5 \mathrm{~mm}$ (0.02 in) thick epoxy at both ends (See Figure 2).

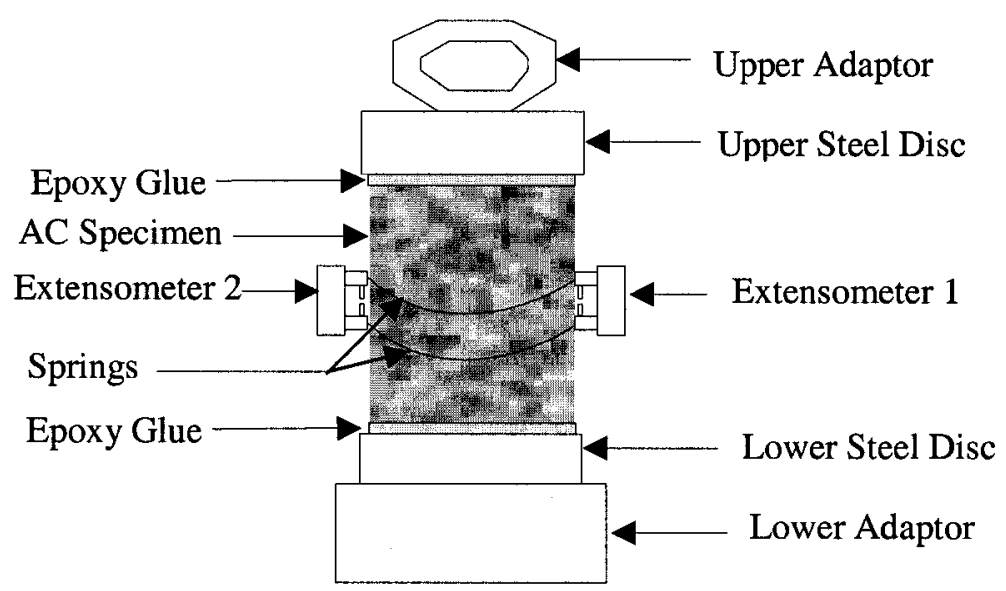

Figure 2: Glued Sample Ready for Testing

6.7 Gyratory Compactor: A gyratory compactor must be used to compact the loose AC material in the laboratory to the specifications that match locally specified air voids content.

\section{Sample Preparation:}

7.1 Mix type: Follow local specifications.

7.2 Binder type: Follow local specifications.

7.3 Binder content: Follow local specifications.

7.4 Size: Size of the specimen should be determined according to ASTM Designation D 3549. For this protocol, samples with a diameter of $100 \mathrm{~mm}$ (4 in) and a height of 100 $\mathrm{mm}(4 \mathrm{in})$ are recommended.

7.5 Mixing: A gyratory compactor should be used to prepare specimens in the lab in accordance with AASHTO TP4.

7.6 Compaction: Specimens should be compacted according to AASHTO TP4 or ASTM D3496 to produce 4-inch height at the targeted air voids percent. 
7.7 Physical Properties: The bulk specific gravity, maximum specific gravity and air voids should be determined according to AASHTO T 166, T 209 and T 269 respectively. Air voids should satisfy local requirements.

7.8 Preparation for Gluing: The ends of all test specimens should be smooth and perpendicular to the axis of the specimen. Preparation of the ends of the specimen should be achieved by sawing with a single or double bladed saw.

7.9 Sample Storage: Completed specimens should be wrapped in polyethylene and stored in an environmentally protected storage area at temperatures between 5 and $25^{\circ} \mathrm{C}$ until the day of the test. To eliminate effects of aging on test results, it is recommended to select a manufacturing date meeting the testing schedule, which should conform with a specified storage period prior to testing for all samples representative of different test conditions.

7.10 Attachment of Specimen to the Steel Discs: The testing specimen should be glued to the hardened steel disc using an epoxy. It should be left for a minimum period of 8 hours prior to testing for the purpose of curing. Pelco LEP 502 Epoxy Glue was found effective in this thesis. The system designed to hold the to glued sample is shown in Figure 3.
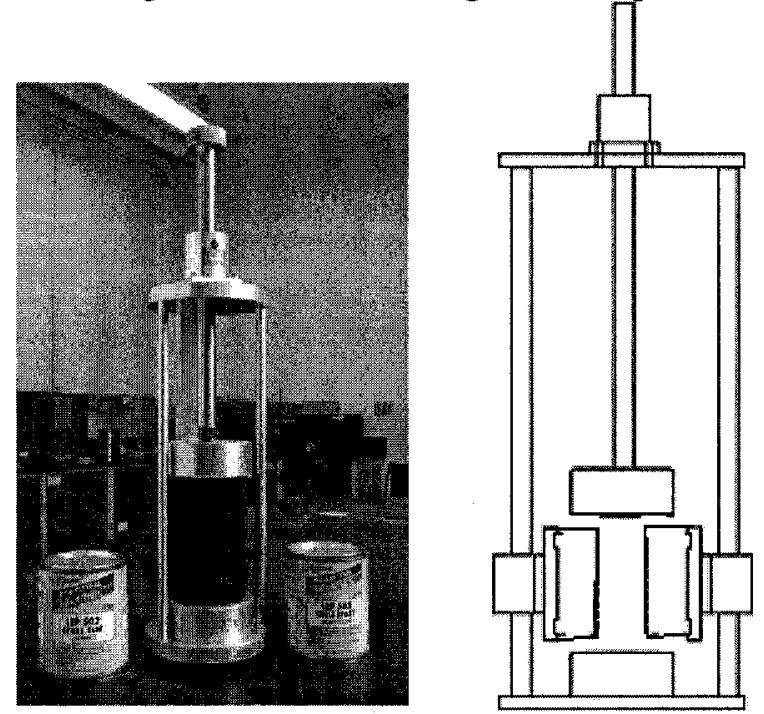

Figure 3: a) System used to glue samples, b) handling system details

7.11 Strain Gauges attachment: Attach the extensometers with springs to the sides of the specimen near mid-height along with a drop of 5-minute epoxy to prevent movements of the extensometers during the test as shown in Figure 2

Note 1: When connecting gauges to specimens with large-size aggregates, care must be taken so that the gauges are attached over areas between the aggregate faces.

7.12 Number of Replicates: A minimum of two specimens is recommended for determining the complex modulus parameters.

\section{Preparation of the Testing System:}

8.1 Calibration: Considering the relatively small stress and strain magnitudes, proper calibration of all sensors connected to the system should be maintained to obtain accurate measurements of the targeted outputs (e.g., displacement or force). Calibration ensures that the outputs of the sensors accurately represent the physical condition sensed by the device. The system software manual provided by the manufacturer should be used as a guide for performing calibration. 
8.2 Tuning: Tuning of the controller extensometer is important for obtaining accurate displacement measurements. Tuning optimizes test performance by minimizing system errors in the assigned control mode. It also improves the response of the machine in order to produce the exact value of input, and it helps in producing a clear signal. The control extensometer should be tuned properly. Retuning the system response may be needed in case the characteristic of the specimen changed. System software manual provided by the manufacturer should be used as a guide for performing tuning. Table 1 shows typical ranges of tuning parameter values recommended for $\mathrm{AC}$ mixes.

Table 1: Typical Extensometer Tuning Parameters for $\mathrm{P}$ and I Gains

\begin{tabular}{|c|c|c|c|c|}
\hline Temperature $\left({ }^{\circ} \mathrm{C}\right)$ & P Gain & I Gain & D Gain & F Gain \\
\hline-10 & $1700-1450$ & $170-145$ & 0.120 & 0.0133 \\
\hline 0 & $1550-1350$ & $155-135$ & 0.120 & 0.0133 \\
\hline 20 & $650-150$ & $65-15$ & 0.120 & 0.0133 \\
\hline 30 & $380-30$ & $38-3$ & 0.120 & 0.0133 \\
\hline 40 & $200-10$ & $20-1$ & 0.120 & 0.0133 \\
\hline
\end{tabular}

8.3 Computer Program for the Test Protocol: The (MPT) software described in section 6.3 should be established to automate the application of the test protocol. The software defines the activities and sequencing involved in running the test (See Table 3 and Figure 4)

The main features of the test protocol include loading frequencies, data acquisition, test control mode, displacement magnitude and rest periods. Table 2 shows the recommended number of cycles needed to arrive at the prescribed strain magnitude $(100 \mu \varepsilon)$ associated with each frequency for testing at different temperatures.

Table 2: Number of Cycles for All Temperature Conditions and Frequencies

\begin{tabular}{|c|c|c|}
\hline Frequency $(\mathrm{Hz})$ & \multicolumn{2}{|c|}{ Number of Cycles } \\
\cline { 2 - 3 } & \multicolumn{2}{|c|}{ Temperature $\left({ }^{\circ} \mathrm{C}\right)$} \\
\cline { 2 - 3 } & $-10,0$ & $20,30,40$ \\
\hline 20 & 1000 & 500 \\
\hline 10 & 500 & 250 \\
\hline 5 & 150 & 75 \\
\hline 1 & 15 & 15 \\
\hline 0.3 & 10 & 10 \\
\hline 0.1 & 5 & 5 \\
\hline
\end{tabular}

A rest period of two minutes between a loading frequency and the other is recommended to enable the machine to release the force applied during a frequency before proceeding to the next as shown in Table 3 and Figure 4.

8.4 Data acquisition system: The data acquisition system should be established to enable automatic collection of data including time, strain as measured by one or two extensometers and the resulting force detected by the load cell at a sampling rate of 100 points per cycle. Data should be collected during the last 50 cycles. Circular buffer type with a size of 5000 points represents a good choice for collecting the last 50 cycles. 
Table 3: Summary of the Complex Modulus Test Tasks

\begin{tabular}{|c|c|}
\hline Task \# & Steps \\
\hline $\begin{array}{l}\text { 1) Condition specimen at }-10^{\circ} \mathrm{C} \text { for } 90 \\
\text { minute }\end{array}$ & $\begin{array}{l}\text { Manually, set the thermal chamber to cool } \\
\text { down the specimen to }-10^{\circ} \mathrm{C}\end{array}$ \\
\hline $\begin{array}{l}\text { 2) Load specimen at } \\
-10^{\circ} \mathrm{C}\end{array}$ & 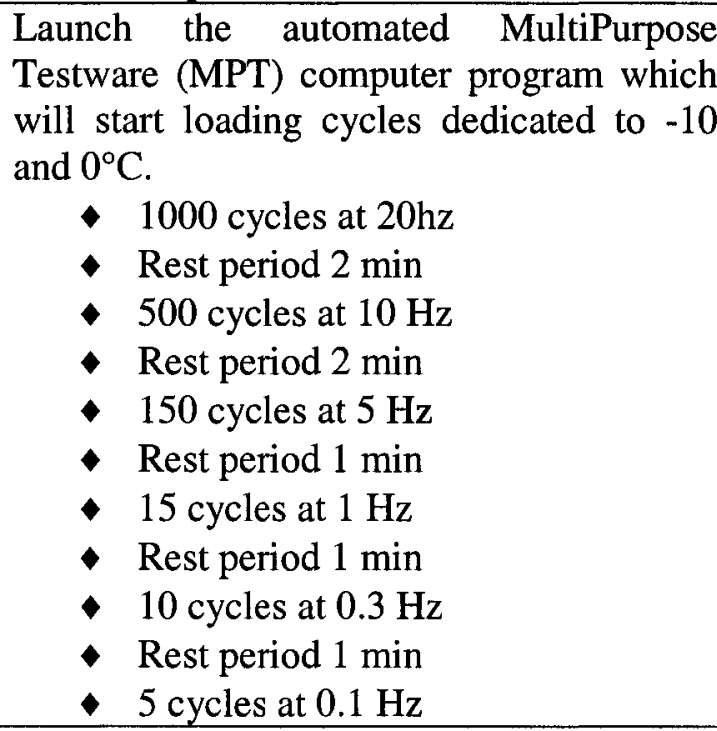 \\
\hline $\begin{array}{l}\text { 3) Condition specimen at } 0^{\circ} \mathrm{C} \text { for } 90 \\
\text { minute }\end{array}$ & $\begin{array}{l}\text { Manually, set the thermal chamber to warm } \\
\text { specimen to } 0^{\circ} \mathrm{C}\end{array}$ \\
\hline 4) Load specimen at $0^{\circ} \mathrm{C}$ & Same as Task 2 \\
\hline $\begin{array}{l}\text { 5) Condition specimen at }+20^{\circ} \mathrm{C} \text { for } 90 \\
\text { minute }\end{array}$ & $\begin{array}{l}\text { Manually, set the thermal chamber to warm } \\
\text { specimen to }+20^{\circ} 1\end{array}$ \\
\hline 6) Testing at $+20^{\circ} \mathrm{C}$ & 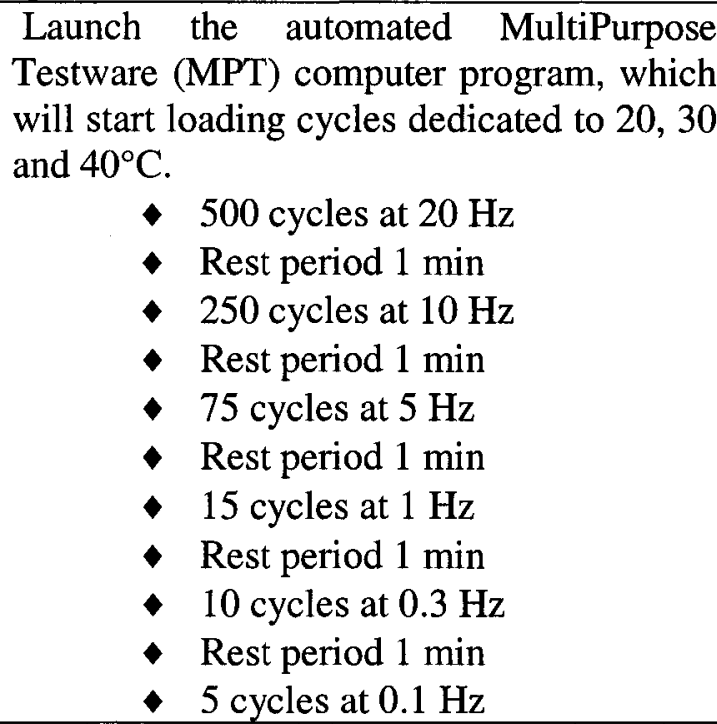 \\
\hline $\begin{array}{l}\text { 7) Conditioning the specimen at } 30^{\circ} \mathrm{C} \\
\text { for } 90 \text { minute }\end{array}$ & $\begin{array}{l}\text { Manually, set the thermal chamber to warm } \\
\text { specimen to }+30^{\circ} \mathrm{C} \text {. }\end{array}$ \\
\hline 8) Load specimen at $+30^{\circ} \mathrm{C}$ & Same as Task 6 \\
\hline $\begin{array}{l}\text { 9) Condition specimen at }+40^{\circ} \mathrm{C} \text { for } 90 \\
\text { minute }\end{array}$ & $\begin{array}{l}\text { Manually, set the thermal chamber to warm } \\
\text { specimen to }+40^{\circ} \mathrm{C} \text {. }\end{array}$ \\
\hline $\begin{array}{l}\text { 10) Load specimen at } \\
+40^{\circ} \mathrm{C}\end{array}$ & Same as Task 6 \\
\hline
\end{tabular}




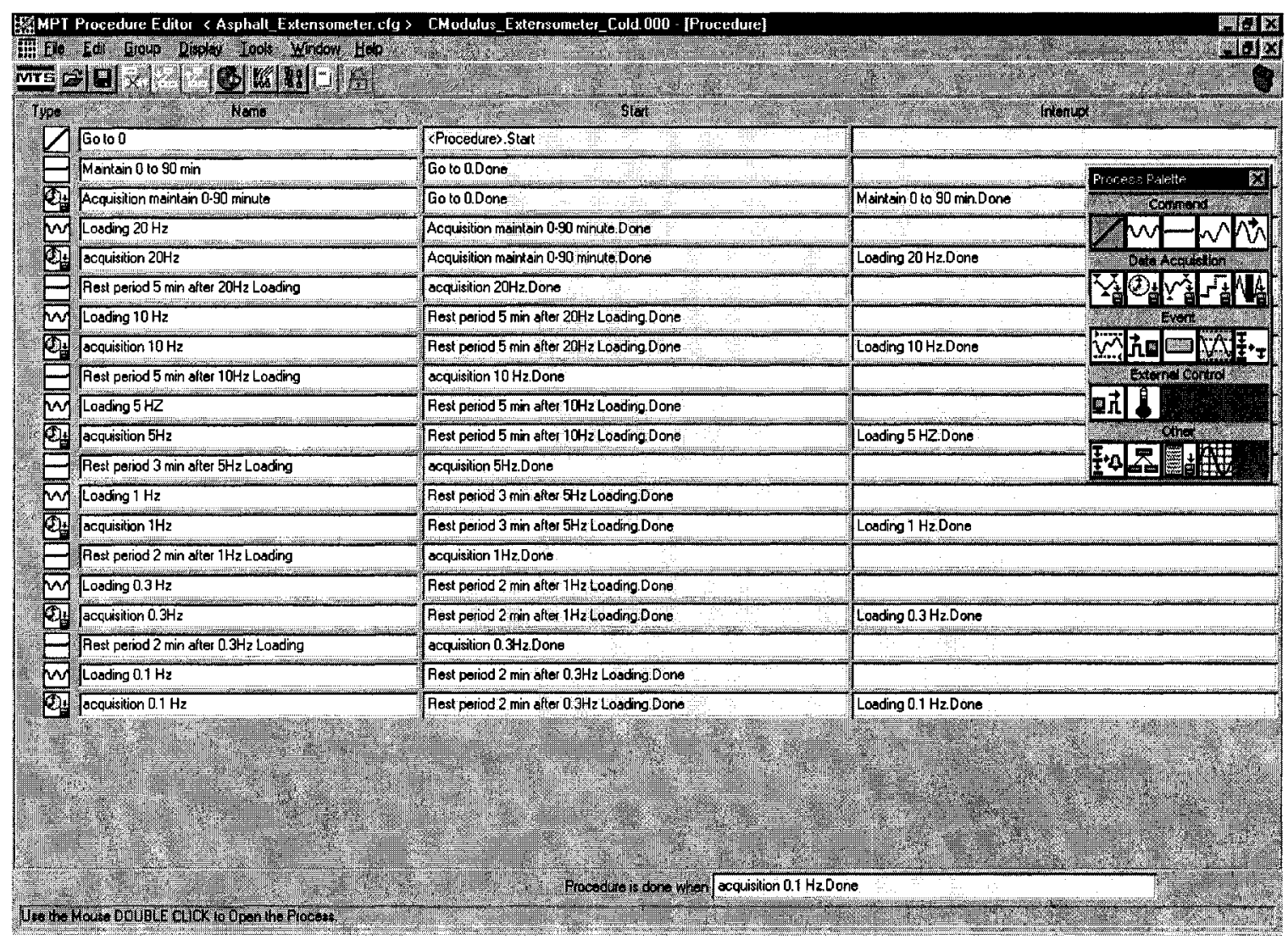

Figure 4: Main window showing the MPT program used to automate the test

\section{Test Procedure:}

The recommended test series consists of five test temperatures $\left(-10,0,20,30\right.$, and $\left.40{ }^{\circ} \mathrm{C}\right)$ and six loading frequencies $(0.1,0.3,1.0,5,10$, and $20 \mathrm{~Hz}$.). Each specimen should be tested for the 30 combinations of temperature and frequency starting with the lowest temperature and proceeding to the highest. Testing at a given temperature should begin with the highest frequency of loading and proceed to the lowest. The step-by-step test procedure involves the following activities: -

9.1 Place the lower adaptor on top of the load cell, and firmly connect them together.

9.2 Place the prepared specimen inside the thermal chamber. Connect the lower adaptor to the lower hardened steel disc already glued to the specimen.

9.3 Connect the upper adaptor to the upper hardened steel disc already glued to the specimen.

9.4 Manually, switch on the thermal chamber to bring the specimen to the specified test temperature.

Note 2: A pre-temperature-equilibrium-test is recommended using a specimen with a thermocouple mounted at the centre to determine the time required by the specimen to reach the specified test temperature. However, a minimum of one and a half hour was founding this thesis to achieve equilibrating at the desired temperature.

9.5 At the end of the sample-conditioning period intended to reach at the equilibrium test temperature, bring the loading actuator in contact with the upper adaptor and connect them firmly. Make sure that they are well centred to avoid eccentricity of the load. Apply 
a small contact load (up to $5 \%$ of the maximum force expected at the specified temperature) to avoid the effect of impact load on the specimen.

9.6 Adjust and balance the electronic measuring system as necessary.

9.7 Automatically launch the automated test protocol described in section 8.3.

9.8 During the test, monitor axial displacement and resulting force on the screen. Make sure that the signals of the applied displacement and corresponding force conform with the input cyclic waveforms. Adjust the recorder chart speed such that at least 3 complete cycles are displayed.

10. Test Output and Data Processing

10.1 Test Output: The data acquisition system collects data automatically at real time (sec) for axial strain as measured by the two extensometers and the resulting force detected by the load cell. Typical data collected is shown in Table 4.

Table 4: Typical data acquired

\begin{tabular}{|c|c|c|c|}
\hline $\begin{array}{c}\text { Time } \\
(\mathrm{Sec})\end{array}$ & $\begin{array}{c}\text { Axial Ram } \\
\text { Force }(\mathrm{N})\end{array}$ & $\begin{array}{c}\text { Axial Ext 1 } \\
(\mathrm{mm})\end{array}$ & $\begin{array}{c}\text { Axial Ext-2 } \\
\mathrm{mm}\end{array}$ \\
\hline 130.427 & -6804.34 & -0.00083 & -0.00023 \\
\hline 130.4275 & -7231.5 & -0.00131 & -0.0004 \\
\hline 130.428 & -7656.24 & -0.00061 & -0.00059 \\
\hline 130.4285 & -8089.91 & -0.00055 & -0.00073 \\
\hline 130.429 & -8510.21 & -0.0009 & -0.00097 \\
\hline 130.4294 & -8878.83 & -0.00104 & -0.00094 \\
\hline 130.4299 & -9243.84 & -0.00156 & -0.00077 \\
\hline
\end{tabular}

\subsection{Data Processing:}

Manual handling of large data files associated with the combination of temperatures and frequencies is time-consuming. To overcome such a difficulty, a macro program based on excel should be developed to facilitate preliminary data reduction by calculating stress and strain amplitudes. The reduced data may be processed further using a statistical package entitled "TableCurve" that assists in fitting different curves and calculating their parameters. This package may be used to fit stress and strain sinusoidal curves produced in the test.

The acquired data, similar to that shown in Table 4 , should be processed to calculate stresses and strains for the last 3 cycles using Equations 1 and 2.

$\sigma_{0}=\frac{\mathrm{P}}{\mathrm{A}}$

Where $\mathrm{A}$ is the cross sectional area of the specimen and $\mathrm{P}$ is the recorded axial load

$\varepsilon_{0}=\frac{\Delta}{\mathrm{L}}$

Where $\Delta$ is the displacement measured by the control extensometer, and $\mathrm{L}$ is the gauge length as shown in Figure 4 


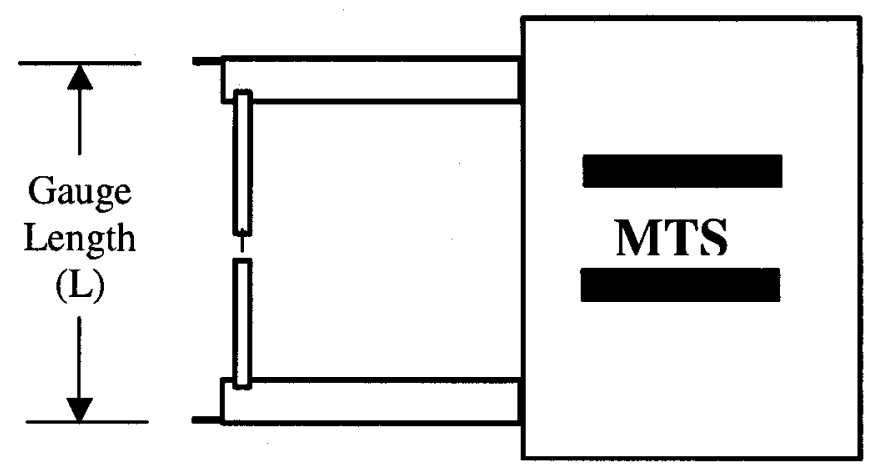

Figure 5: Axial Extensometer Model 632.11F - 90

Stresses and strains are then plotted against time to determine stress $\left(\sigma_{0}\right)$ and strain amplitudes $\left(\varepsilon_{0}\right)$ as shown in Figure 5.

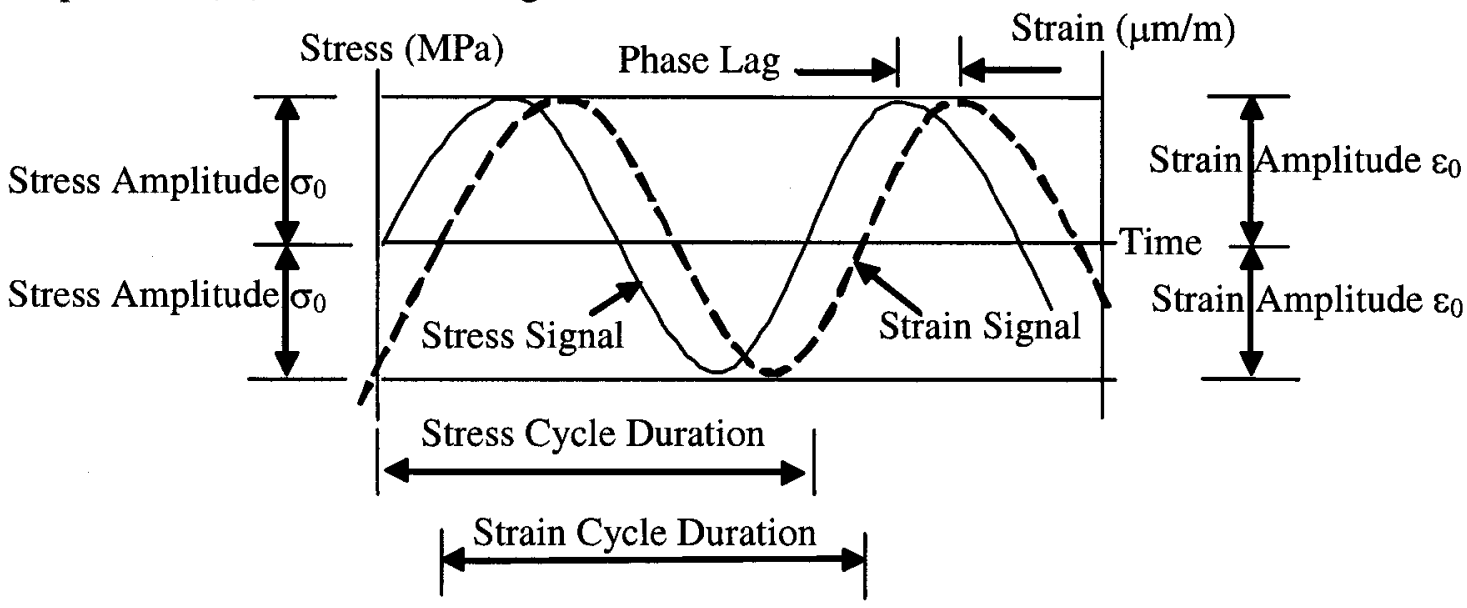

Figure 6: Strain and Stress vs. Time

\section{Dynamic Modulus and Phase Angle Determination}

11.1 Determine the phase lag $\left(T_{i}\right)$ between the stress peak and the strain peak values from Figure 6.

11.2 Calculate the phase angle using Equation 3 as follows:

$\phi=\frac{T_{i}}{T_{0}} \times 360^{\circ}$

Where:

$T_{i}=$ Time lag between the stress and strain cycles $(\mathrm{sec})$

$T_{p}=$ time for a stress cycle (sec.)

11.3 Calculate the dynamic modulus, $\left|E^{*}\right|$ using Equation 4:

$\left|E^{*}\right|=\frac{\sigma_{0}}{\varepsilon_{0}}$

Note 3: - There are several methods for determining the stresses, strains, and the phase angles. These methods include peak search algorithms; curve fitting techniques, and Fourier Transform. However, curve-fitting techniques have a significant advantage over others methods in determining the amplitudes of stresses, strains and the phase angles. These parameters can be found easily using waveform equations that replace Equations 1 , 2, and 3 as shown in Equations 5 and 6 as follows: 


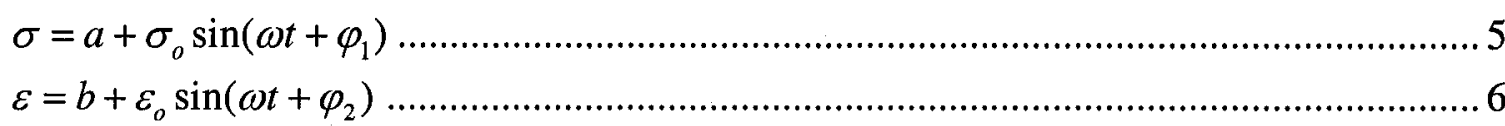

Where:

$\sigma$ and $\varepsilon$ are the stress and strain respectively at time $t$,

$\sigma_{o}$ and $\varepsilon_{0}$ are the amplitude of stress and strain respectively,

$\omega=2 \pi f$, the Angular velocity in radians, $f$ is the frequency in $\mathrm{Hz}$,

$a$ and $b$ are regression constants,

$\varphi_{1}, \varphi_{2}$ Represent individual phase angles of stress and strain wave functions respectively.

The phase lag $\phi$ between stress and strain cycles is calculated as the difference of $\left(\varphi_{1}-\varphi_{2}\right)$ in radian

11.4 Calculate the storage modulus $\mathrm{E}_{1}$ using Equation 7:

$\mathrm{E}_{1}=\left|E^{*}\right| \cos \phi$

11.8 Calculate the loss modulus using Equation 8:

$\mathrm{E}_{2}=\left|E^{*}\right| \sin \phi$

12. Presentation of the mechanistic properties:

This test protocol includes two forms of data presentation that will satisfy requirements of two common technique of analysis including:

a- Input data for models that uses the dynamic modulus to establish a master curve that models the behaviour of the AC material. A typical example of such models is the AASHTO 2002 Design Guide.

b- Huet-Sayegh parameters that models the rheological behaviour of AC materials.

\subsection{Mechanistic Properties Required by the AASHTO 2002 Design Guide:}

The primary mix-related property of interest by the Design Guide software for an AC layer is the dynamic modulus $\left|E^{*}\right|$ measured at a number of loading frequencies and test temperatures. Laboratory-determined values of dynamic modulus are required as inputs in Level 1 of the 2002 Design Guide. Actual laboratory test data are required to develop the master curve and shift factors used to describe the behavior of AC layer. The laboratory data requirements for Level 1 analysis are summarized in Table 5.

\begin{tabular}{|c|c|c|c|c|c|c|}
\hline Temperature, ${ }^{\circ} \mathrm{C}$ & \multicolumn{6}{|c|}{ Dynamic Modulus $\left|E^{*}\right|,[\mathrm{MPa}]$} \\
\hline & $20 \mathrm{~Hz}$ & $10 \mathrm{~Hz}$ & $5 \mathrm{~Hz}$ & $1 \mathrm{~Hz}$ & $0.3 \mathrm{~Hz}$ & $0.1 \mathrm{~Hz}$ \\
\hline-10 & $\mathrm{X}$ & $X$ & $\mathrm{X}$ & $\mathrm{X}$ & $\mathrm{X}$ & $\mathrm{X}$ \\
\hline 0 & $\mathrm{X}$ & $\mathrm{X}$ & $X$ & $\mathrm{X}$ & $X$ & $X$ \\
\hline 20 & $\mathrm{X}$ & $\mathrm{X}$ & $X$ & $\mathrm{X}$ & $X$ & $X$ \\
\hline 30 & $\mathrm{X}$ & $\mathrm{X}$ & $X$ & $\mathrm{X}$ & $\mathrm{X}$ & $\mathrm{X}$ \\
\hline 40 & $\mathrm{X}$ & $X$ & $X$ & $X$ & $\mathrm{X}$ & $\mathrm{X}$ \\
\hline
\end{tabular}

\subsection{Calculations of Rheological Parameters:}

Results of the complex modulus test (dynamic moduli and phase angles), calculated at a number of temperatures and frequencies combinations, may be utilized to calculate rheological parameters that can be used in Huet-Sayegh model to describe AC behaviour. The Huet-Sayegh model is given by Equation 9. 


$$
E^{*}(i \omega)=E_{0}+\frac{E_{\infty}-E_{0}}{1+\delta(i \omega \tau)^{-k}+(i \omega \tau)^{-h}}
$$

The model consists of eight parameters. Five of them are expressed explicitly in Equation 9, namely:

$E_{0}:$ is the high temperature stiffness,

$E_{\infty}:$ is the purely elastic modulus,

$\delta, k$, and $h$ : are characteristics of the parabolic elements. They define the linearviscoelastic response of pavement materials.

The other three parameters are determined implicitly using $\tau$, which is referred to as the "characteristics time" and it is calculated using Equation 10:

$\ln (\tau)=a+b T+c T^{2}$

Where $a, b$, and $c$ are regression constants representing material characteristics.

Four parameters of the Huet-Sayegh model including $E_{0}, E_{\infty}, k$, and $h$ can be determined from the graphical representation shown in Figures 7 and 8. However, the other four parameters including regression constants $\mathrm{a}, \mathrm{b}$, and $\mathrm{c}$ in addition to $\delta$ are determined iteratively to achieve the best fit that can be obtained in the Cole-Cole and Black diagrams.

\section{Cole-Cole Diagram:}

The diagram is the result of plotting the storage modulus $E_{1}$ versus the loss modulus $E_{2}$. Figure 7 shows typical data plotted in cole-cole space.

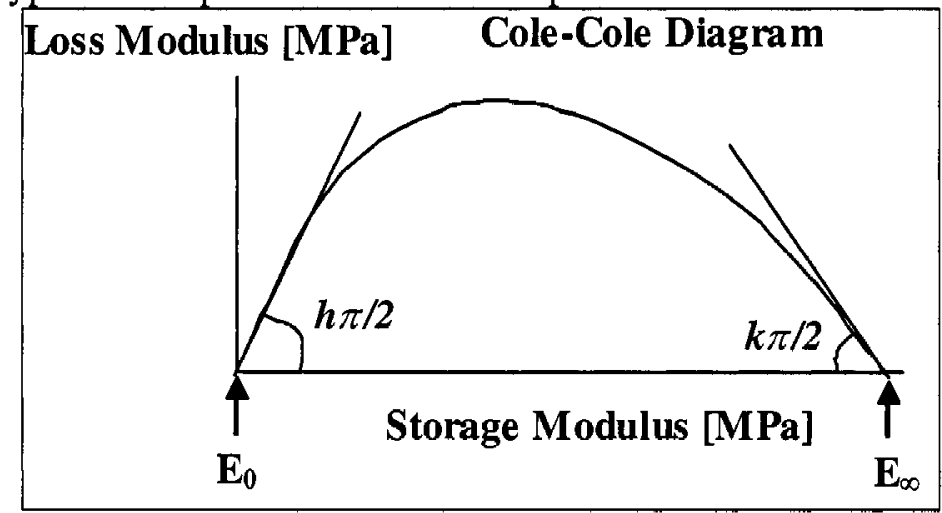

Figure 7: Graphical Representation of Sayegh Model Parameters in Cole-Cole

\section{Black Diagram:}

Diagarm

This diagram illustrates the relationship between the dynamic modulus, $\left|E^{*}\right|$ and the phase angle $\phi$. Figure 8 shows typical data plotted in Black space. 


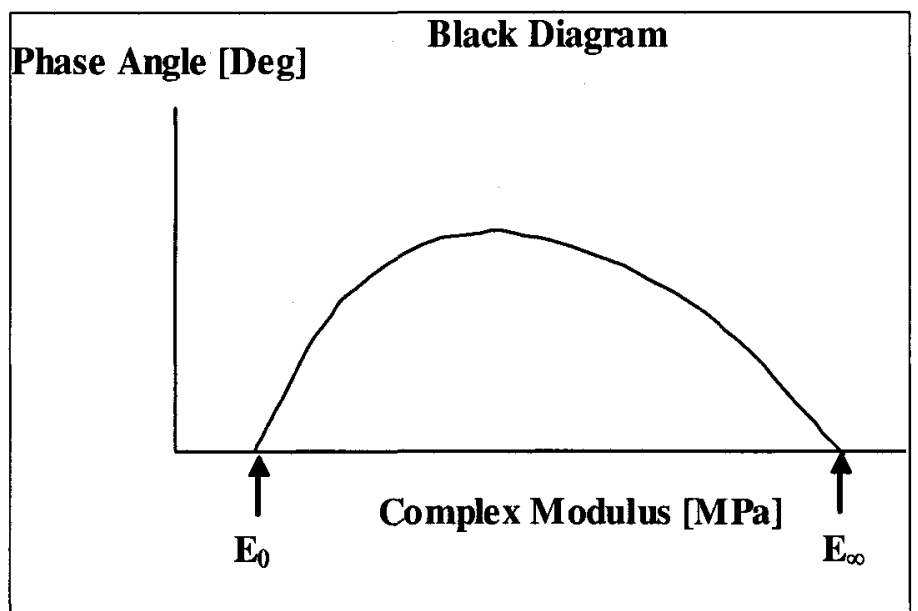

Figure 8: Graphical Representation of Hue-Sayegh Model Parameters Black Space

Note 4: HUSAROAD program, a part of VEROAD Software developed by the "Netherlands Pavement Consultants" is commercially available to assist in fitting laboratory data and determining Huet-Sayegh model parameters.

13. Test Report Form: the recommended format of reporting the test results include physical properties of the tested specimen and the mechanical properties including the dynamic modulus and phase angles in a set, and the 8 parameters of Huet-Sayegh model as shown below.

Mix Identification:

Mix Category:

Local Classification:

Sample ID:

Physical Properties of the Mix:

Nominal Maximum Aggregate Size mm (in):

Binder Type:

Binder Content (\%)

Air Voids Content (\%):

Mechanical Properties:

Dynamic Modulus and Phase Angle

\begin{tabular}{|c|c|c|c|c|c|c|c|}
\hline \multicolumn{8}{|c|}{ Dynamic Modulus [MPa] and Phase Angle, $\left[{ }^{\circ}\right]$} \\
\hline 7 & 7 & 20 & 10 & 5 & 1 & 0.3 & 0.1 \\
\hline \multirow[b]{2}{*}{-10} & $\left|E^{*}\right|$ & & & & & & \\
\hline & $\phi$ & & & & & & \\
\hline \multirow[b]{2}{*}{0} & $\left|E^{*}\right|$ & & & & & & \\
\hline & $\phi$ & & & & & & \\
\hline \multirow[b]{2}{*}{20} & $\left|\mathrm{E}^{*}\right|$ & & & & & & \\
\hline & $\phi$ & & & & & & \\
\hline \multirow[b]{2}{*}{30} & $\left|E^{*}\right|$ & & & & & & \\
\hline & $\phi$ & & & & & & \\
\hline \multirow[b]{2}{*}{40} & $\mathrm{E}^{*}$ & & & & & & \\
\hline & $\phi$ & & & & & & \\
\hline
\end{tabular}


Huet-Sayegh Model Parameters:

\begin{tabular}{|l|l|l|l|l|l|l|l|l|}
\hline \multirow{3}{*}{ Replicate \# } & \multicolumn{9}{|l|}{ Model Coefficients } & $\begin{array}{l}E_{\infty} \\
(\mathrm{MPa})\end{array}$ & $\begin{array}{l}E_{0} \\
(\mathrm{MPa})\end{array}$ & $k$ & $h$ & $\delta$ & \multicolumn{3}{|l|}{$\begin{array}{l}\text { Characteristic } \\
\text { Coefficients }\end{array}$} \\
\hline & & & & & & & & \\
\hline 1 & & & & & & & & \\
\hline 2 & & & & & & & & \\
\hline
\end{tabular}




\section{APPENDIX B}

ASPHALT CONCRETE MATERIAL LIBRARY 


\section{Preface:}

The asphalt concrete properties produced in this thesis are summarized in the following tables.

They are intended to form a part of a large pavement Material Library including all materials used to construct roads. Some of the properties are provided by the manufacturers and are were verified in this study.

In the Library, the appropriate table for the desired properties should be identified in Table 1:The Directory". The specific line within that table is also identified in Table 1 and is refereed to as ID

\section{Definitions of Terminologies:}

Terminologies used in the Material Library are defined as follows:

$\mathrm{E}_{0}=$ High temperature stiffness

$\mathrm{E}_{\infty}=$ the purely elastic modulus

$\delta, k$, and $h$ are parameters of the biparabolic elements of the model

$\tau=$ Parameter referred to as the characteristics time, it is a temperature dependent variable

$p_{a b s}=$ Percentage asphalt absorption

$p_{a c}=$ Percentage asphalt content, by weight of mix

$p_{\text {opt }}=$ Optimum asphalt content of mix, in percent by weight of mix

$\eta=$ Bitumen viscosity, in $10^{6}$ poise,

$f=$ Loading frequency, in $\mathrm{Hz}$,

$\mathrm{V}_{\mathrm{a}}=$ Percent air voids in the mix, by volume,

$\mathrm{V}_{\text {beff }}$ Percent effective bitumen content, by volume,

$\mathrm{R} 3 / 4, \mathrm{P}_{34}=$ Percent retained on $3 / 4$-inch sieve, by total aggregate weight (cumulative),

$\mathrm{R} 3 / 8, \mathrm{P}_{38}=$ Percent retained on 3/8-inch sieve, by total aggregate weight (cumulative),

$\mathrm{R} \# 4, \mathrm{P}_{4}=$ Percent retained on No. 4 sieve, by total aggregate weight (cumulative), and

$\mathrm{P}_{200}=$ Percent passing No. 200 sieve, by total aggregate weight.

$\mathrm{mPa} . \mathrm{s}=$ Mega Pascal times second

$\mathrm{rad} / \mathrm{sec}=$ radians per second

$\mathrm{kPa}=$ kilo Pascal

$\mathrm{D}_{\text {nom }}=$ Nominal maximum aggregate size, $\mathrm{mm}$

$\mathrm{D}_{\max }=$ Maximum aggregate size, $\mathrm{mm}$

HL 8 = an MTO mix designation used as Stabilized base course or binder course

HL 4 = an MTO mix designation used as a binder course

HL 3 = an MTO mix designation used as a surface course

HMA 1 = A mix designation used in this study to represent $\mathrm{HL} 8 \mathrm{mix}$

HMA 2 = A mix designation used in this study to represent HL 4 mix 
HMA 3 = A mix designation used in this study to represent HL 3 mix

SP $1=$ A mix designation used in this study to represent a SuperPave mix design with $D_{\text {nom }}$ of 19 $\mathrm{mm}$

SP 2 = A mix designation used in this study to represent a SuperPave mix design with $D_{\text {nom }}$ of $12.5 \mathrm{~mm}$

$\mathrm{VMA}=$ Voids in mineral aggregate as a percent of bulk volume of compacted mix

VFA $=$ Voids Filled with Asphalt, $\%$

Agg $\%=$ percent of aggregates content by total mass of sample

$\mathrm{AC} \%, \mathrm{P}_{\mathrm{b}}=$ Binder content by total mass of a loose mixture

$\mathrm{P}_{\mathrm{be}}=$ Effective Binder Content, \% by volume

$\mathrm{P}_{0.075}=$ Percent of Aggregates Passing Sieve \# 200

MSG, $\mathrm{G}_{\mathrm{mm}}=$ Maximum specific gravity of a loose mixture

$\mathrm{G}_{\mathrm{b}}, \mathrm{G}_{\mathrm{mb}}, \mathrm{BSG}=$ Bulk specific gravity of compacted mixture

$\left[{ }^{\circ}\right]=$ Angle unit in degrees

$\mathrm{N}=$ Newton

$\mathrm{A}=$ Cross-sectional area of compacted $\mathrm{AC}$ specimen, $\mathrm{mm}^{2}$

$\mathrm{P}=$ Axial force, $\mathrm{N}$

$\Delta=$ Displacement as measured by the extensometer, $\mathrm{mm}$

$\mathrm{L}=$ Gauge length of the extensometer used to control the test

$S$. Point $=$ softening point of asphalt binder

A. Viscosity $=$ absolute viscosity of asphalt binder

$\mathrm{K}$. Viscosity = kinematic viscosity of asphalt binder

Pen. = penetration of asphalt binder

$\mathrm{R}$. Viscosity $=$ rotational viscosity of asphalt binder

Gse $=$ Effective specific gravity of aggregates

$\mathrm{Gsb}=$ Bulk specific gravity of aggregates 
MATERIAL LIBRARY

\section{ASPHALT CONCRETE CHARACTERISTICS}

\section{Lead Table}

\begin{tabular}{|c|c|c|c|c|c|c|c|}
\hline \multicolumn{2}{|c|}{ Mix ID } & \multicolumn{2}{|c|}{ Binder } & \multirow{2}{*}{$\begin{array}{c}\text { Aggregates } \\
\text { Aggregate Properties } \\
\text { Table \# } 3 \\
\end{array}$} & \multicolumn{3}{|c|}{ AC Mix } \\
\hline \multirow{2}{*}{$\begin{array}{l}\text { Designation } \\
\text { used in this } \\
\text { Study }\end{array}$} & \multirow{2}{*}{$\begin{array}{c}\text { Local } \\
\text { Designation }\end{array}$} & \multirow{2}{*}{\begin{tabular}{|c|} 
Binder Type \\
(Properties \\
Shown in \\
Table \# 2)
\end{tabular}} & \multirow{2}{*}{$\begin{array}{c}\text { Binder } \\
\text { Content, } \\
\%\end{array}$} & & $\begin{array}{c}\text { Physical Properties } \\
\text { Table \#4 }\end{array}$ & $\begin{array}{c}\text { Huet-Syegh } \\
\text { Parameters -Table \# 5 } \\
\end{array}$ & $\begin{array}{r}\text { Dynamic Modulus } \\
\text { and Phase Angles } \\
\end{array}$ \\
\hline & & & & \begin{tabular}{|c|} 
Aggregate \\
ID \\
\end{tabular} & \begin{tabular}{|c|} 
Physical Properties \\
ID
\end{tabular} & HS_ID & Table \# \\
\hline HMA1 & HL8 & \begin{tabular}{|l|} 
PG52-34 \\
\end{tabular} & 4.5 & 1 & 1 & 1 & 6 \\
\hline HMA1 & HL8 & PG58-22 & 4.5 & 1 & 2 & 2 & 7 \\
\hline HMA2 & HL4 & PG52-34 & 5.0 & 2 & 3 & 3 & 8 \\
\hline HMA2 & HL4 & PG58-22 & 5.0 & 2 & 4 & 4 & 9 \\
\hline HMA2 & HLA & PG64-34 & 5.0 & 2 & 5 & 5 & 10 \\
\hline HMA3 & HL3 & PG52-34 & 5.0 & 3 & 6 & 6 & 11 \\
\hline HMA3 & HL3 & PG58-22 & 5.0 & 3 & 7 & 7 & 12 \\
\hline HMA3 & HL3 & PG64-34 & 5.0 & 3 & 8 & 8 & 13 \\
\hline SP 1 & SP 1 & PG52-34 & 4.5 & 4 & 9 & 9 & 14 \\
\hline SP 1 & SP 1 & PG58-22 & 4.5 & 4 & 10 & 10 & 15 \\
\hline SP 2 & SP 2 & PG52-34 & 4.5 & 5 & 11 & 11 & 16 \\
\hline SP 2 & SP 2 & PG64-34 & 4.5 & 5 & 13 & 13 & 18 \\
\hline
\end{tabular}

Table 2: Binder Properties

\begin{tabular}{|c|c|c|c|c|c|c|}
\hline Binder Type & $\begin{array}{c}\text { Bulk Specific } \\
\text { Gravity }\end{array}$ & $\begin{array}{c}\text { Softening } \\
\text { Point }\end{array}$ & $\begin{array}{c}\text { Absolute } \\
\text { Viscosity }\end{array}$ & $\begin{array}{c}\text { Kinematics } \\
\text { Viscosity }\end{array}$ & $\begin{array}{c}\text { Rotational } \\
\text { Viscosity }\end{array}$ \\
\hline PG52-34 & 1.020 & 125 & 340 & 2200 & 1 & 0.266 \\
\hline PG58-22 & 1.025 & 125 & 340 & 2200 & 2 \\
\hline PG64-34 & 1.028 & 125 & 340 & 2200 & 3 & 0.323 \\
\hline
\end{tabular}


Table 3: Aggregates Properties

\begin{tabular}{|c|c|c|c|c|c|}
\hline $\begin{array}{c}\text { Aggregate } \\
\text { ID }\end{array}$ & R3/4" & R3/8" & R.\#4 & P\#200 & $\begin{array}{c}\text { Bulk Specific Gravity } \\
\text { (Gsb) }\end{array}$ \\
\hline 1 & 3.5 & 46.2 & 51.4 & 0.5 & 2.740 \\
\hline 2 & 0.0 & 35.0 & 40.9 & 0.7 & 2.742 \\
\hline 3 & 0.0 & 20.7 & 41.0 & 0.5 & 2.720 \\
\hline 4 & 12.8 & 56.6 & 59.2 & 0.3 & 2.721 \\
\hline 5 & 5.0 & 24.3 & 32.1 & 0.9 & 2.755 \\
\hline
\end{tabular}

Table 4: Mix Physical Properties

\begin{tabular}{|c|c|c|c|c|c|c|c|}
\hline $\begin{array}{l}\text { Mix Physical } \\
\text { Properties ID }\end{array}$ & $\begin{array}{c}\text { Maximum } \\
\text { Specific Gravity }\end{array}$ & Bulk Specific Gravity & $\begin{array}{c}\text { Effective } \\
\text { Specific Gravity }\end{array}$ & $\begin{array}{l}\text { Air Voids } \\
\text { Content, \% }\end{array}$ & \begin{tabular}{|c|} 
Voids in Mineral \\
Aggregates, \%
\end{tabular} & $\begin{array}{c}\text { Voids Filled } \\
\text { with Asphalt, \% }\end{array}$ & \begin{tabular}{|c|} 
Effective Binder \\
Content, $\%$
\end{tabular} \\
\hline 1 & 2.566 & 2.429 & 2.760 & 5.3 & 15.3 & 65.2 & 10.0 \\
\hline 2 & 2.540 & 2.412 & 2.728 & 5.0 & 15.9 & 68.4 & 10.9 \\
\hline 3 & 2.524 & 2.402 & 2.733 & 4.8 & 16.8 & 71.2 & 12.0 \\
\hline 4 & 2.536 & 2.421 & 2.747 & 4.5 & 17.3 & 71.9 & 11.6 \\
\hline 5 & 2.506 & 2.389 & 2.715 & 4.7 & 16.1 & 73.1 & 12.7 \\
\hline 6 & 2.503 & 2.375 & 2.702 & 5.1 & 17.0 & 69.9 & 11.9 \\
\hline 7 & 2.530 & 2.396 & 2.740 & 5.3 & 16.3 & 67.6 & 11.0 \\
\hline 8 & 2.477 & 2.337 & 2.675 & 5.7 & 18.4 & 69.3 & 12.7 \\
\hline 9 & 2.547 & 2.418 & 2.737 & 5.1 & 15.1 & 66.5 & 10.1 \\
\hline 10 & 2.544 & 2.415 & 2.733 & 5.1 & 15.2 & 66.7 & 10.2 \\
\hline 11 & 2.570 & 2.441 & 2.765 & 5.0 & 15.4 & 67.4 & 10.4 \\
\hline 12 & 2.577 & 2.434 & 2.773 & 6.0 & 15.6 & 64.5 & 10.1 \\
\hline 13 & 2.590 & 2.435 & 2.789 & 5.6 & 15.6 & 61.6 & 9.6 \\
\hline
\end{tabular}


Table 5: Huet-Sayegh Model Parameters

\begin{tabular}{|c|c|c|c|c|c|c|c|c|}
\hline HS_ID & $\mathrm{E}_{\text {inf }}$ & $\mathrm{E}_{0}$ & $\delta$ & $\mathrm{K}$ & $\mathrm{h}$ & $\mathrm{a}$ & $\mathrm{b}$ & $\mathrm{c}$ \\
\hline 1 & 26813 & 187 & 2.42 & 0.25 & 0.59 & -2.4 & -0.35 & 0.003 \\
\hline 2 & 16440 & 60 & 1.48 & 0.19 & 0.6 & 0.87 & -0.29 & 0.001 \\
\hline 3 & 24967 & 33 & 2.98 & 0.28 & 0.62 & -1.77 & -0.28 & 0.001 \\
\hline 4 & 36812 & 188 & 3.43 & 0.28 & 0.63 & 1.49 & 0.32 & 0.001 \\
\hline 5 & 17726 & 25 & 2.8 & 0.29 & 0.56 & -1.27 & -0.28 & 0.001 \\
\hline 6 & 24983 & 17 & 2.74 & 0.26 & 0.59 & -1.66 & -0.23 & 0 \\
\hline 7 & 28932 & 68 & 2.08 & 0.25 & 0.61 & 1.07 & -0.36 & 0 \\
\hline 8 & 23715 & 35 & 2.79 & 0.24 & 0.57 & -2.6 & -0.3 & 0.002 \\
\hline 9 & 29466 & 34 & 2.42 & 0.28 & 0.6 & -1.77 & -0.35 & 0.003 \\
\hline 10 & 28557 & 93 & 1.57 & 0.2 & 0.56 & 0.59 & -0.34 & 0.003 \\
\hline 11 & 31441 & 59 & 2.58 & 0.29 & 0.59 & -0.97 & -0.36 & 0.003 \\
\hline 12 & 32666 & 86 & 1.96 & 0.22 & 0.56 & 1.83 & -0.33 & 0.001 \\
\hline 13 & 28373 & 127 & 2.32 & 0.23 & 0.53 & -0.31 & 0.27 & 0.001 \\
\hline
\end{tabular}

Table 6: HMA 1, PG 52-34

\begin{tabular}{|c|c|c|c|c|c|c|c|}
\hline \multicolumn{2}{|c|}{$\underset{\mathrm{T}\left[{ }^{\circ} \mathrm{C}\right]}{\text { Frequency }[\mathrm{Hz}]}$} & \multirow{2}{*}{$\frac{20}{17511}$} & \multirow{2}{*}{$\frac{10}{16639}$} & \multirow{2}{*}{$\begin{array}{c}5 \\
15999 \\
\end{array}$} & \multirow{2}{*}{$\frac{1}{13305}$} & \multirow{2}{*}{$\frac{0.3}{11095}$} & \multirow{2}{*}{$\frac{0.1}{8555}$} \\
\hline$-10 \mathrm{C}$ & $\left|\mathrm{E}^{*}\right|$ & & & & & & \\
\hline & $\phi$ & 8.5 & 9 & 10 & 13.4 & 16.9 & 20.2 \\
\hline \multirow[t]{2}{*}{$0 \mathrm{C}$} & $\left|E^{*}\right|$ & 9965 & 8611 & 7515 & 5264 & 3835 & 2770 \\
\hline & $\phi$ & 17.3 & 19.6 & 21.6 & 26.3 & 29.8 & 31.2 \\
\hline \multirow{2}{*}{20} & $F^{*} !$ & 1025 & 1475 & 137 & 672 & 475 & 375 \\
\hline & $\phi$ & 38.9 & 39.1 & 38.4 & 35.3 & 31.5 & 27.5 \\
\hline \multirow[t]{2}{*}{30} & $\left|E^{*}\right|$ & 871 & 660 & 520 & 349 & 289 & 267 \\
\hline & $\phi$ & 42.5 & 39.7 & 36.1 & 26.6 & 18.8 & 12.5 \\
\hline & & & & & & & \\
\hline \multirow[t]{2}{*}{40} & $\left|\mathrm{E}^{*}\right|$ & 435 & 378 & 337 & 276 & 258 & 258 \\
\hline & $\phi$ & 36.2 & 29.5 & 23.7 & 15.5 & 11.2 & 10.8 \\
\hline
\end{tabular}


Table 7: HMA 1, PG 58-22

\begin{tabular}{|c|c|c|c|c|c|c|c|}
\hline \multicolumn{2}{|c|}{ Frequency [Hz] } & & & & & & \\
$\mathrm{T}\left[{ }^{\circ} \mathrm{C}\right]$ & & 20 & 10 & 5 & 1 & 0.3 & 0.1 \\
\hline$-10 \mathrm{C}$ & $\left|\mathrm{E}^{*}\right|$ & 12597 & 12356 & 11816 & 10803 & 10060 & 9255 \\
\hline & $\phi$ & 3.6 & 3.8 & 4.3 & 6 & 7 & 9 \\
\hline & & & & & & & \\
\hline $0 \mathrm{C}$ & $\left|\mathrm{E}^{*}\right|$ & 9978 & 9675 & 8777 & 7478 & 6479 & 5617 \\
\hline & $\phi$ & 7.5 & 8 & 9.6 & 11.7 & 14.1 & 16.9 \\
\hline & & & & & & & \\
\hline 20 & $\left|\mathrm{E}^{*}\right|$ & 7025 & 6158 & 5464 & 4080 & 3171 & 2484 \\
\hline & $\phi$ & 13.6 & 15.3 & 17.2 & 21.5 & 24.9 & 28.1 \\
\hline & & & & & & & \\
\hline 30 & $\left|\mathrm{E}^{*}\right|$ & 2585 & 1925 & 1475 & 800 & 504 & 342 \\
\hline & $\phi$ & 35.1 & 38.2 & 40.1 & 43.2 & 43.8 & 41.8 \\
\hline & & & & & & & \\
\hline 40 & $\left|\mathrm{E}^{*}\right|$ & 883 & 632 & 467 & 239 & 158 & 121 \\
\hline & $\phi$ & 45.8 & 47 & 46.7 & 44.6 & 41.6 & 33.7 \\
\hline
\end{tabular}

Table 8: HMA 2, PG 52-34

\begin{tabular}{|c|c|c|c|c|c|c|c|}
\hline \multicolumn{2}{|l|}{$\begin{array}{l}\text { Frequency } \\
\mathrm{T}\left[{ }^{\circ} \mathrm{C}\right]\end{array}$} & \multicolumn{2}{l|}{} & & & & \\
\hline$-10 \mathrm{C}$ & $\left.\mid \mathrm{E}^{*}\right\rfloor$ & 14796 & 13618 & 12571 & 9966 & 8310 & 6885 \\
\hline & $\phi$ & 10.9 & 12.2 & 13.7 & 16.3 & 19.4 & 21.4 \\
\hline & & & & & & & \\
\hline $0 \mathrm{C}$ & $\mid \mathrm{E}^{*}$ & 10044 & 8546 & 7452 & 5158 & 3810 & 2802 \\
\hline & $\phi$ & 18.1 & 20.6 & 22.2 & 26.3 & 29.4 & 31.6 \\
\hline & & & & & & & \\
\hline 20 & $\mid \mathrm{E}^{*}$ & 2075 & 1553 & 1174 & 647 & 421 & 290 \\
\hline & $\phi$ & 40.5 & 42 & 42.5 & 42.7 & 42 & 40.4 \\
\hline & & & & & & & \\
\hline 30 & $\mid \mathrm{E}^{*}$ & 954 & 675 & 490 & 246 & 153 & 108 \\
\hline & $\phi$ & 46.7 & 47.7 & 47.6 & 45.9 & 43.9 & 40.9 \\
\hline & & & & & & & \\
\hline 40 & $\mid \mathrm{E}^{*}$ & 336 & 235 & 171 & 100 & 68 & 58 \\
\hline & $\phi$ & 51.1 & 50.9 & 49.2 & 43.6 & 38.7 & 31.2 \\
\hline
\end{tabular}


Table 9: HMA 2, PG 58-22

\begin{tabular}{|c|c|c|c|c|c|c|c|}
\hline \multicolumn{2}{|c|}{ Frequency $[\mathrm{Hz}]$} & & & & & & \\
$\mathrm{T}\left[{ }^{\circ} \mathrm{C}\right]$ & 20 & 10 & 5 & 1 & 0.3 & 0.1 \\
\hline$-10 \mathrm{C}$ & $\left|\mathrm{E}^{*}\right|$ & 29697 & 29173 & 30000 & 29500 & 28500 & 27400 \\
\hline & $\phi$ & 5.5 & 6 & 6.8 & 7.9 & 9 & 9.8 \\
\hline & & & & & & & \\
\hline $0 \mathrm{C}$ & $\left|\mathrm{E}^{*}\right|$ & 23917 & 21462 & 19581 & 15985 & 13130 & 10345 \\
\hline & $\phi$ & 9.5 & 10 & 11.1 & 14.9 & 19.1 & 22.1 \\
\hline & & & & & & & \\
\hline 20 & $\left|\mathrm{E}^{*}\right|$ & 7654 & 6212 & 4943 & 3073 & 2070 & 1390 \\
\hline & $\phi$ & 25.5 & 27.9 & 31.1 & 34.7 & 37.1 & 37.3 \\
\hline & & & & & & & \\
\hline 30 & $\left|\mathrm{E}^{*}\right|$ & 3120 & 2355 & 1777 & 980 & 655 & 470 \\
\hline & $\phi$ & 37.7 & 39.7 & 40.8 & 39.8 & 37.9 & 34 \\
\hline & & & & & & & \\
\hline 40 & $\mid \mathrm{E}^{*}$ & 1313 & 985 & 752 & 470 & 365 & 320 \\
\hline & $\phi$ & 43.4 & 42.1 & 29.3 & 26.6 & 30.8 & 24.6 \\
\hline
\end{tabular}

Table 10: HMA 2, PG 64-34 - Mechanical Properties

\begin{tabular}{|c|c|c|c|c|c|c|c|}
\hline \multicolumn{2}{|c|}{ Frequency [Hz] } & & & & & & \\
$\mathrm{T}\left[{ }^{\circ} \mathrm{C}\right]$ & & 20 & 10 & 5 & 1 & 0.3 & 0.1 \\
\hline$-10 \mathrm{C}$ & $\left|\mathrm{E}^{*}\right|$ & 11654 & 10537 & 9662 & 7937 & 6786 & 5804 \\
\hline & $\phi$ & 9.2 & 10.8 & 11.5 & 14.2 & 16.2 & 18.2 \\
\hline & & & & & & & \\
\hline $0 \mathrm{C}$ & $\left|\mathrm{E}^{*}\right|$ & 8401 & 7206 & 6301 & 4683 & 3617 & 2813 \\
\hline & $\phi$ & 15.2 & 17.5 & 19 & 22.4 & 25.1 & 27.2 \\
\hline & & & & & & & \\
\hline 20 & $\left|\mathrm{E}^{*}\right|$ & 4167 & 3362 & 2784 & 1808 & 1275 & 933 \\
\hline & $\phi$ & 26.3 & 28.6 & 30 & 33 & 34.9 & 35.5 \\
\hline & & & & & & & \\
\hline 30 & $\left|\mathrm{E}^{*}\right|$ & 901 & 664 & 504 & 286 & 200 & 150 \\
\hline & $\phi$ & 42.8 & 43 & 41.5 & 39.1 & 36.9 & 34.5 \\
\hline & & & & & & & \\
\hline 40 & $\left|\mathrm{E}^{*}\right|$ & 342 & 252 & 189 & 113 & 83 & 63 \\
\hline & $\phi$ & 44.6 & 44.1 & 41.4 & 36 & 33.8 & 30.3 \\
\hline
\end{tabular}

Table 11: HMA 3, PG 52-34 - Mechanical Properties

\begin{tabular}{|c|c|c|c|c|c|c|c|}
\hline \multicolumn{2}{|c|}{ Frequency [Hz] } & & & & & & \\
$\mathrm{T}\left[{ }^{\circ} \mathrm{C}\right]$ & & 20 & 10 & 5 & 1 & 0.3 & 0.1 \\
\hline$-10 \mathrm{C}$ & $\left|\mathrm{E}^{*}\right|$ & 15138 & 13312 & 11979 & 9568 & 8021 & 6783 \\
\hline & $\phi$ & 10.1 & 11.8 & 13.3 & 15.5 & 17.9 & 20 \\
\hline & & & & & & & \\
\hline $0 \mathrm{C}$ & $\left|\mathrm{E}^{*}\right|$ & 10013 & 8459 & 7384 & 5342 & 4083 & 3079 \\
\hline & $\phi$ & 17.1 & 19.3 & 21.1 & 24.8 & 27.7 & 30.4 \\
\hline & & & & & & & \\
\hline 20 & $\left|\mathrm{E}^{*}\right|$ & 3422 & 2592 & 2054 & 1244 & 842 & 579 \\
\hline & $\phi$ & 32.4 & 34.7 & 36.3 & 38.5 & 39.3 & 39.1 \\
\hline & & & & & & & \\
\hline 30 & $\left|\mathrm{E}^{*}\right|$ & 932 & 656 & 486 & 263 & 171 & 121 \\
\hline & $\phi$ & 46.6 & 47.7 & 48.2 & 47.1 & 45.6 & 44 \\
\hline & & & & & & & \\
\hline 40 & $\left|\mathrm{E}^{*}\right|$ & 412 & 291 & 212 & 121 & 96 & 67 \\
\hline & $\phi$ & 48.3 & 48.4 & 47.6 & 44.7 & 43.2 & 39.9 \\
\hline
\end{tabular}


Table 12: HMA 3, PG 58-22- Mechanical Properties

\begin{tabular}{|c|c|c|c|c|c|c|c|}
\hline \multicolumn{2}{|c|}{ Frequency $[\mathrm{Hz}]$} & & & & & & \\
$\mathrm{T}\left[{ }^{\circ} \mathrm{C}\right]$ & & 20 & 10 & 5 & 1 & 0.3 & 0.1 \\
\hline$-10 \mathrm{C}$ & $\left|\mathrm{E}^{*}\right|$ & 24957 & 24330 & 23193 & 21496 & 19737 & 18008 \\
\hline & $\phi$ & 3.4 & 4 & 4.9 & 6.1 & 7.4 & 9.2 \\
\hline & & & & & & & \\
\hline $0 \mathrm{C}$ & $\left|\mathrm{E}^{*}\right|$ & 19716 & 18138 & 16877 & 13612 & 11267 & 9233 \\
\hline & $\phi$ & 8.8 & 9.7 & 10.8 & 13.9 & 16.9 & 19.6 \\
\hline & & & & & & & \\
\hline 20 & $\left|\mathrm{E}^{*}\right|$ & 10288 & 9118 & 8043 & 5617 & 4108 & 2783 \\
\hline & $\phi$ & 20.8 & 23.4 & 26.2 & 31.2 & 35.4 & 38.6 \\
\hline & & & & & & & \\
\hline 30 & $\left|\mathrm{E}^{*}\right|$ & 3176 & 2336 & 1723 & 874 & 525 & 342 \\
\hline & $\phi$ & 40.1 & 42.1 & 44.1 & 47.3 & 48 & 46 \\
\hline & & & & & & & \\
\hline 40 & $\left|\mathrm{E}^{*}\right|$ & 1193 & 825 & 600 & 294 & 183 & 142 \\
\hline & $\phi$ & 49.1 & 50.1 & 45 & 43.4 & 38.2 & 36.8 \\
\hline
\end{tabular}

Table 13: HMA 3, PG 64-34 - Mechanical Properties

\begin{tabular}{|c|c|c|c|c|c|c|c|}
\hline \multicolumn{2}{|c|}{ Frequency $[\mathrm{Hz}]$} & & & & & & \\
$\mathrm{T}\left[{ }^{\circ} \mathrm{C}\right]$ & & 20 & 10 & 5 & 1 & 0.3 & 0.1 \\
\hline$-10 \mathrm{C}$ & $\left|\mathrm{E}^{*}\right|$ & 13280 & 11700 & 11034 & 9364 & 7376 & 5833 \\
\hline & $\phi$ & 10.9 & 12.1 & 13.6 & 16.1 & 19.4 & 21.7 \\
\hline & & & & & & & \\
\hline $0 \mathrm{C}$ & $\left|\mathrm{E}^{*}\right|$ & 7973 & 6870 & 5928 & 4111 & 3068 & 2285 \\
\hline & $\phi$ & 16 & 20.2 & 22.1 & 26.3 & 29 & 31.1 \\
\hline & & & & & & & \\
\hline 20 & $\left|\mathrm{E}^{*}\right|$ & 1644 & 1246 & 969 & 529 & 342 & 246 \\
\hline & $\phi$ & 39.5 & 40.6 & 41.6 & 41.9 & 40.9 & 40 \\
\hline & & & & & & & \\
\hline 30 & $\left|\mathrm{E}^{*}\right|$ & 694 & 509 & 387 & 205 & 138 & 100 \\
\hline & $\phi$ & 45 & 45.5 & 44.8 & 42.4 & 39.6 & 36.7 \\
\hline & & & & & & & \\
\hline 40 & $\left|\mathrm{E}^{*}\right|$ & 322 & 234 & 179 & 102 & 75 & 58 \\
\hline & $\phi$ & 46.5 & 45.6 & 27.1 & 36.9 & 31.8 & 30.8 \\
\hline
\end{tabular}

Table 14: SP 1, PG 52-34 - Mechanical Properties

\begin{tabular}{|c|c|c|c|c|c|c|c|}
\hline \multicolumn{2}{|c|}{ Frequency [Hz] } & & & & & & \\
$\mathrm{T}\left[{ }^{\circ} \mathrm{C}\right]$ & & 20 & 10 & 5 & 1 & 0.3 & 0.1 \\
\hline$-10 \mathrm{C}$ & $\left|\mathrm{E}^{*}\right|$ & 21267 & 19774 & 18686 & 15515 & 13245 & 11005 \\
\hline & $\phi$ & 8 & 9 & 10 & 13.1 & 16.6 & 18.4 \\
\hline & & & & & & & \\
\hline $0 \mathrm{C}$ & $\left|\mathrm{E}^{*}\right|$ & 12783 & 11040 & 9675 & 6892 & 5145 & 3825 \\
\hline & $\phi$ & 17.3 & 19.4 & 21.4 & 26.1 & 30.1 & 32.5 \\
\hline & & & & & & & \\
\hline 20 & $\left|\mathrm{E}^{*}\right|$ & 2438 & 1781 & 1317 & 716 & 437 & 285 \\
\hline & $\phi$ & 41.8 & 44 & 45.2 & 45.6 & 45.8 & 44.9 \\
\hline & & & & & & & \\
\hline 30 & $\left|\mathrm{E}^{*}\right|$ & 1174 & 835 & 599 & 303 & 188 & 125 \\
\hline & $\phi$ & 47 & 48.1 & 48.3 & 47.2 & 45.5 & 42.9 \\
\hline & & & & & & & \\
\hline 40 & $\left|\mathrm{E}^{*}\right|$ & 459 & 322 & 235 & 120 & 80 & 60 \\
\hline & $\phi$ & 51 & 50.7 & 36.3 & 41.6 & 45.7 & 41.2 \\
\hline
\end{tabular}


Table 15: SP 1, PG 58-22 - Mechanical Properties

\begin{tabular}{|c|c|c|c|c|c|c|c|}
\hline \multicolumn{2}{|c|}{ Frequency [Hz] } & & & & & & \\
$\mathrm{T}\left[{ }^{\circ} \mathrm{C}\right]$ & 20 & 10 & 5 & 1 & 0.3 & 0.1 \\
\hline$-10 \mathrm{C}$ & $\left|\mathrm{E}^{*}\right|$ & 23071 & 22154 & 21828 & 20273 & 18655 & 17065 \\
\hline & $\phi$ & 3.9 & 4.7 & 5.1 & 6.3 & 7.9 & 9.2 \\
\hline & & & & & & & \\
\hline $0 \mathrm{C}$ & $\left|\mathrm{E}^{*}\right|$ & 17754 & 16759 & 15762 & 13132 & 11270 & 9490 \\
\hline & $\phi$ & 7.7 & 9 & 10 & 12.9 & 16.9 & 19.9 \\
\hline & & & & & & & \\
\hline 20 & $\left|\mathrm{E}^{*}\right|$ & 7490 & 6000 & 4829 & 2908 & 1895 & 1225 \\
\hline & $\phi$ & 24.2 & 28.5 & 31.9 & 37.5 & 40.7 & 41.9 \\
\hline 30 & $\left|\mathrm{E}^{*}\right|$ & 3810 & 2890 & 2194 & 1225 & 810 & 560 \\
\hline & $\phi$ & 36.4 & 38.7 & 39.7 & 40.1 & 39 & 36.7 \\
\hline & $\left|\mathrm{E}^{*}\right|$ & 1616 & 1180 & 871 & 475 & 320 & 240 \\
\hline 40 & $\phi$ & 44.3 & 44.9 & 36.1 & 37.6 & 39.7 & 33.7 \\
\hline & $\phi$ & & & & & &
\end{tabular}

Table 16: SP 2, PG 52-34 - Mechanical Properties

\begin{tabular}{|c|c|c|c|c|c|c|c|}
\hline \multicolumn{2}{|c|}{ Frequency $[\mathrm{Hz}]$} & & & & & & \\
$\mathrm{T}\left[{ }^{\circ} \mathrm{C}\right]$ & 20 & 10 & 5 & 1 & 0.3 & 0.1 \\
\hline$-10 \mathrm{C}$ & $\left|\mathrm{E}^{*}\right|$ & 20313 & 19158 & 16904 & 14535 & 12890 & 11460 \\
\hline & $\phi$ & 7.2 & 8.7 & 9.5 & 11.0 & 13.2 & 14.7 \\
\hline & & & & & & & \\
\hline $0 \mathrm{C}$ & $\left|\mathrm{E}^{*}\right|$ & 14271 & 12679 & 10217 & 8340 & 6805 & 5275 \\
\hline & $\phi$ & 11.0 & 13.0 & 14.3 & 17.4 & 19.9 & 21.9 \\
\hline 20 & $\left|\mathrm{E}^{*}\right|$ & 5671 & 4755 & 3871 & 2609 & 1955 & 1515 \\
\hline & $\phi$ & 27.2 & 30.3 & 31.4 & 32.2 & 32.3 & 31.2 \\
\hline & & & & & & \\
\hline 30 & $\left|\mathrm{E}^{*}\right|$ & 2550 & 1947 & 1534 & 974 & 715 & 565 \\
\hline & $\phi$ & 37.6 & 39.0 & 37.8 & 35.4 & 33.4 & 31.1 \\
\hline & & & & & & & \\
\hline 40 & $\left|\mathrm{E}^{*}\right|$ & 1109 & 853 & 674 & 455 & 345 & 285 \\
\hline & $\phi$ & 39.5 & 39.0 & 28.5 & 30.4 & 33.7 & 27.8 \\
\hline
\end{tabular}

Table 17: SP 2, PG 58 -22 - Mechanical Properties

\begin{tabular}{|c|c|c|c|c|c|c|c|}
\hline \multicolumn{2}{|c|}{ Frequency $[\mathrm{Hz}]$} & & & & & & \\
$\mathrm{T}\left[{ }^{\circ} \mathrm{C}\right]$ & & 20 & 10 & 5 & 1 & 0.3 & 0.1 \\
\hline$-10 \mathrm{C}$ & $\left|\mathrm{E}^{*}\right|$ & 24777 & 23880 & 23382 & 21265 & 17200 & 13785 \\
\hline & $\phi$ & 6.0 & 7.0 & 7.4 & 9.4 & 14.3 & 17.6 \\
\hline & & & & & & & \\
\hline $0 \mathrm{C}$ & $\left|\mathrm{E}^{*}\right|$ & 14821 & 12747 & 10984 & 7682 & 5525 & 3895 \\
\hline & $\phi$ & 15.8 & 17.6 & 20.4 & 25.6 & 29.9 & 32.6 \\
\hline & & & & & & & \\
\hline 20 & $\left|\mathrm{E}^{*}\right|$ & 3024 & 2253 & 1692 & 945 & 615 & 420 \\
\hline & $\phi$ & 39.3 & 41.6 & 42.3 & 41.9 & 40.4 & 37.6 \\
\hline 30 & $\left|\mathrm{E}^{*}\right|$ & 1190 & 870 & 635 & 337 & 225 & 170 \\
\hline & $\phi$ & 47.7 & 48.1 & 47.1 & 43.6 & 40.0 & 38.7 \\
\hline & & & & & & & \\
\hline 40 & $\left|\mathrm{E}^{*}\right|$ & 457 & 323 & 241 & 140 & 105 & 85 \\
\hline & $\phi$ & 46.6 & 39.2 & 33.8 & 31.4 & 28.7 & 24.1 \\
\hline
\end{tabular}


Table 18: SP 2, PG 64 -34 - Mechanical Properties

\begin{tabular}{|c|c|c|c|c|c|c|c|}
\hline \multicolumn{2}{|c|}{ Frequency $[\mathrm{Hz}]$} & & & & & & \\
$\mathrm{T}\left[{ }^{\circ} \mathrm{C}\right]$ & & 20 & 10 & 5 & 1 & 0.3 & 0.1 \\
\hline$-10 \mathrm{C}$ & $\left|\mathrm{E}^{*}\right|$ & 28278 & 27593 & 27244 & 25213 & 23015 & 20985 \\
\hline & $\phi$ & 3.2 & 3.3 & 3.6 & 5.1 & 7.5 & 8.6 \\
\hline & & & & & & & \\
\hline $0 \mathrm{C}$ & $\left|\mathrm{E}^{*}\right|$ & 23171 & 21657 & 20676 & 17650 & 14990 & 12090 \\
\hline & $\phi$ & 6.8 & 7.2 & 8.6 & 12.1 & 15.7 & 18.6 \\
\hline & & & & & & & \\
\hline 20 & $\left|\mathrm{E}^{*}\right|$ & 10150 & 8647 & 7273 & 4860 & 3470 & 2435 \\
\hline & $\phi$ & 19.8 & 22.7 & 25.5 & 30.4 & 33.4 & 34.5 \\
\hline & & & & & & & \\
\hline 30 & $\left|\mathrm{E}^{*}\right|$ & 4183 & 3144 & 2368 & 1288 & 825 & 575 \\
\hline & $\phi$ & 35.6 & 38.7 & 40.4 & 41.5 & 40.4 & 38.4 \\
\hline & & & & & & & \\
\hline 40 & $\mid \mathrm{E}^{*}$ & 1732 & 1240 & 898 & 480 & 315 & 235 \\
\hline & $\phi$ & 45.2 & 46 & 34.4 & 39.2 & 45.4 & 39.6 \\
\hline
\end{tabular}




\section{APPENDIX C \\ MACRO FOR REDUCING COMPLEX MODULUS TEST DATA}




Dim sFileName As String

Dim iDelemiterPosition As Integer

Dim sPathToFiles As String

Dim sDirectory As String

Dim iNumberOfPoints As Integer

Dim fSampleDiameter As Single, fSampleLength As Single

Dim iNumberOfBlocks As Integer, ipoints As Integer

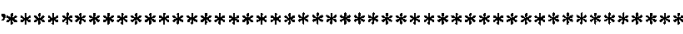

Sub getPathAndPrefix()

Dim s As String

$\mathrm{s}=\mathrm{sDirectory}$

iDelemiterPosition $=1$

ReDim Preserve sFileName(iDelemiterPosition)

sFileName(iDelemiterPosition) $=\mathrm{s}$

Do While Len(s)

Debug.Print StrGetToken(s, "l")

iDelemiterPosition $=$ iDelemiterPosition +1

ReDim Preserve sFileName(iDelemiterPosition)

sFileName(iDelemiterPosition) $=\mathrm{s}$

Loop

sTempFileName $=$ getPrefix $($ sFileName $($ iDelemiterPosition 1))

sPathToFiles $=$ getPath(sFileName $(1)$, sTempFilePrefix $)$

End Sub

Function getPath(sName As String, sDelimiter As String) As

String

Dim $\mathrm{nPos}$ As Long

Dim sToken As String

Check for position of delimeter

nPos $=$ InStr(sName, sDelimiter)

'If delimeter is found, strip off token

If $(\mathrm{nPos}>0)$ Then

getPath $=$ Left\$(sName, $n$ Pos - 1)

Else

getPath $=$ sName

End If

End Function

Function getPrefix(sName As String) As String

Dim nPos As Long

Dim sToken As String

Check for position of delimeter

nPos = InStr(sName, "I")

'If delimeter is found, strip off token

If (nPos > 0) Then

getPrefix $=$ Left $\$($ sName, $n$ Pos -1$)$

Else

getPrefix $=$ sName

End If

End Function

Public Function StrGetToken(ByRef psString As

String, ByVal psDelim As String) As String

Dim nPos As Long

Dim sToken As String

On Error GoTo ERROR_Handler

sToken $=$ psString

'Check for empty string

If (Len(psString)) Then

'Check for position of delimeter

nPos $=$ InStr(psString, psDelim)

'If delimeter is found, strip off token

If ( $\mathrm{nPos}>0$ ) Then

sToken $=$ Left\$ $(p s S t r i n g, n$ Pos -1$)$

'Strip token and delimiter from

'string passed in

psString $=$ Mid $\$(p s S t r i n g, n P o s+$ Len $($ psDelim $))$

Else

'No delimiter found, set string

'passed into an empty string

psString = "”
End If

End If

StrGetToken $=$ sToken

Exit Function

ERROR Handler:

StrGetToken $=$ "'"

End Function

Private Sub cmdCancel_Click()

Call UserForm_Terminate

End Sub

Private Sub cmdDefaults_Click()

Cells $(100,30)$.Value $=$ frmParameters.txtDirectory. Text

Cells $(101,30)$. Value $=$ frmParameters.txtFileName.Text

Cells $(102,30)$.Value $=$ frmParameters.txtNoOfPoints. Text

Cells $(103,30)$. Value $=$

frmParameters.txtSampleDiameter.Text

Cells $(104,30)$.Value $=$ frmParameters.txtSampleLength. Text

Cells $(105,30)$.Value $=$ frmParameters.txtPoints. Text

End Sub

Private Sub cmdNew_Click()

Dim s As String

Dim iCount As Integer, i As Integer, $\mathrm{j}$ As Integer

Dim iNumOfFiles As Integer

Dim s_sheet As String

On Error Resume Nex

Call sortAndRenameSheet

'now delete any pre exist sheets (Note access the sheets by

names not by index)

Application. DisplayAlerts $=$ False

iCount $=$ Sheets . Count

If iCount $>1$ Then

For $\mathrm{i}=2$ To iCount

$\mathrm{s}=$ "sheet" \& LTrim(Str\$(i))

Sheets(s).Delete

Next

End If

'close all opened files

Call CloseAllWorkbookExceptActive

'enable all controls

Call EnableControls

End Sub

Sub EnableControls()

frmParameters. $\mathrm{cmdOk}$. Enabled $=$ True

frmParameters txtDirectory. Enabled $=$ True

frmParameters. txtFileName . Enabled $=$ True

frmParameters.txtNoOfPoints. Enabled $=$ True

frmParameters. txtSampleDiameter. Enabled $=$ True

frmParameters. txtSampleLength. Enabled $=$ True

frmParameters.txtPoints. Enabled $=$ True

End Sub

Private Sub cmdOk_Click()

sDirectory $=$ frmParameters.txtDirectory. Text

sFileName $=$ frmParameters.txtFileName.Text

fSampleDiameter $=$

Val(frmParameters.txtSampleDiameter.Text)

fSampleLength = Val(frmParameters.txtSampleLength.Text) iNumberOfPoints $=$ Val(frmParameters.txtNoOfPoints. Text $)$ ipoints $=$ Val(frmParameters.txtPoints. Text)

Application.ScreenUpdating $=$ False

Call getApplicationName

'disable all controls

Call DisableControls

Call addNewSheet

Call AnalyzeData(sDirectory, sFileName, iNumberOfPoints,

fSampleDiameter, fSampleLength, ipoints)

Call UserForm_Terminate

Application.ScreenUpdating $=$ True

End Sub

Sub DisableControls() 
frmParameters. .

frmParameters.txtDirectory. Enabled $=$ False

frmParameters.txtFileName .Enabled $=$ False

frmParameters.txtNoOfPoints. Enabled $=$ False

frmParameters.txtSampleDiameter.Enabled $=$ False

frmParameters.txtSampleLength.Enabled $=$ False

frmParameters.txtPoints. Enabled $=$ False

End Sub

Sub sortAndRenameSheets()

Dim iCount As Integer

Dim s As String

iCount $=$ Sheets. Count

If iCount $>1$ Then

'sort worksheets in a workbook in ascending order

For $\mathrm{i}=1$ To iCount -1

For $j=i+1$ To iCount

If Sheets(j).Name < Sheets(i).Name Then

Sheets $(\mathrm{j})$.Move Before: $=$ Sheets $(\mathrm{i})$

End If

Next $j$

Next i

'rename each sheet to an arbitrary name

For $\mathrm{i}=2$ To iCount

$\mathrm{s}=$ "s999" \& LTrim(Str\$(i))

Sheets $(\mathrm{i}) \cdot$ Name $=\mathrm{s}$

Next i

'rename each sheet to a standard name

For $\mathrm{i}=2$ To iCount

$\mathrm{s}=$ "sheet" \& LTrim(Str\$(i))

Sheets(i).Name $=s$

Next $i$

End If

End Sub

Sub addNewSheet()

Dim i As Integer

iNumberOfBlocks $=$ getNumberOfBlocks

For $\mathrm{i}=1$ To iNumberOfBlocks +1

Sheets("Sheet1").Select

Sheets.Add

Next

Call sortAndRenameSheets

End Sub

Private Sub txtUpdate_Click()

sDirectory $=$ frmParameters.txtDirectory. Text

sTempFileName $=$ frmParameters.txtFileName.Text

iNumberOfPoints $=$ frmParameters.txtFileName.Text

fSampleDiameter $=$

Val(frmParameters.txtSampleDiameter.Text)

fSampleLength $=\mathrm{Val}$ (frmParameters.txtSampleLength.Text $)$

ipoints $=$ Val (frmParameters.txtPoints. Text)

End Sub

Private Sub UserForm_Initialize()

Call DisableControls

If (IsEmpty(Cells $(100,30)$.Value)) Then

frmParameters.txtDirectory.Text = "D:/NRC_Yassin_Macro" Else

frmParameters.txtDirectory.Text $=\operatorname{Cells}(100,30)$. Value

End If

If (IsEmpty(Cells (101, 30).Value)) Then

frmParameters.txtFileName.Text = "specimen.dat"

Else

frmParameters.txtFileName. Text $=$ Cells $(101,30)$. Value

End If

If (IsEmpty(Cells(102, 30).Value)) Then

frmParameters.txtNoOfPoints. Text $=$ iNumberOfPoints

Else

frmParameters.txtNoOfPoints. Text $=\operatorname{Cells}(102,30)$, Value

End If

If (IsEmpty(Cells(103, 30).Value)) Then

frmParameters.txtSampleDiameter.Text $=$ fSampleDiameter
Else

frmParameters.txtSampleDiameter. Text $=$ Cells $(103$,

30). Value

End If

If (IsEmpty(Cells(104, 30).Value)) Then

frmParameters.txtSampleLength. Text $=$ fSampleLength

Else

frmParameters.txtSampleLength.Text $=\operatorname{Cells}(104,30)$.Value

End If

If (IsEmpty(Cells(105, 30).Value)) Then

frmParameters.txtPoints. Text $=$ ipoints

Else

frmParameters.txtPoints. Text $=$ Cells $(105,30)$. Value

End If

End Sub

Private Sub UserForm_Terminate()

frmParameters. Hide

Set frmParameters $=$ Nothing

End

End Sub

Option Explicit

Const iNumberOfBlocks As Integer $=6$

Const iTimeColumn As Integer $=1$

Const iForceColumn As Integer $=2$

Const iExt1Column As Integer $=3$

Const iExt2Column As Integer $=4$

Const iExt3Column As Integer $=5$

Dim sApplicationName As String

Dim iNumberOfPointsPerBlock As Integer

Dim fFrequency (1 To iNumberOfBlocks) As Single

Type MaxAndMinRange

fMinValue As Single

fMaxValue As Single

iRowNumberOfMaxValue As Integer

End Type

Sub ShowForm()

frmParameters. Show

End Sub

Sub getApplicationName()

sApplicationName $=$ ActiveWorkbook. Name

End Sub

Function getNumberOfBlocks()

getNumberOfBlocks $=$ iNumberOfBlocks

End Function

Sub AnalyzeData(sDirectory As String, sFileName As

String, N As Integer, fDiameter As Single, fLength As

Single, ipoints As Integer)

Dim s As String

Dim i As Integer

iNumberOfPointsPerBlock $=\mathbf{N}$

Windows(sApplicationName).Activate

Sheets("Sheet2").Select

Cells $(1,1)$.Select

If Right(sDirectory, 1) = "l" Then

$s=$ Directory \& sFileName

Else

$\mathrm{s}=$ sDirectory \& "I" \& sFileName

End If

Workbooks.OpenText Filename:=s,

Origin:=xIWindows, StartRow: $=1$,

DataType: $=x$ 1Delimited, TextQualifier:=

xlDoubleQuote, ConsecutiveDelimiter:=True, Tab:=True, Semicolon:=True,

Comma: $=$ True, Space:=True, Other:=False,

FieldInfo: $=$ Array $(\operatorname{Array}(1,1), \ldots \operatorname{Array}(2$,

1), $\operatorname{Array}(3,1), \operatorname{Array}(4,1), \operatorname{Array}(5,1), \operatorname{Array}(6,1)$,

$\operatorname{Array}(7,1), \operatorname{Array}(8,1), \operatorname{Array}(9$,

1), $\operatorname{Array}(10,1), \operatorname{Array}(11,1), \operatorname{Array}(12,1), \operatorname{Array}(13,1)$,

$\operatorname{Array}(14,1), \operatorname{Array}(15,1)$,

$\operatorname{Array}(16,1), \operatorname{Array}(17,1), \operatorname{Array}(18,1), \operatorname{Array}(19,1))$ 
Windows(sFileName).Activate

Call ComputeStresses(sFileName, N, fDiameter, fLength) Workbooks(sFileName).Close

DrawChartForEachBlock

Call ComputePhaseLag(ipoints)

Call InsertTitles

Call InsertTime

End Sub

Sub InsertTime()

Dim fValueOfFirstTempReading As Single

Dim iStartOfBlock As Integer, i As Integer, $\mathrm{j}$ As Integer

iStartOfBlock $=2$

For $\mathrm{i}=1$ To iNumberOfBlocks

fValueOfFirstTempReading $=$ Cells $($ iStartOfBlock,

1).Value

For $\mathbf{j}=1$ To iNumberOfPointsPerBlock

Cells(iStartOfBlock, 9). Value = Cells(iStartOfBlock,

1).Value -

fValueOfFirstTempReading

iStartOfBlock $=$ iStartOfBlock +1

Next

iStartOfBlock $=$ iStartOfBlock +1

Next

End Sub

Sub InsertTitles()

Cells $(1,1) \cdot$ Value $=$ "Time"

Cells $(1,2)$. Value $=$ "Force $(\mathrm{N}) "$

Cells $(1,3)$. Value $=" \operatorname{Ext1}(\mathrm{mm}) "$

Cells $(1,4)$. Value $=$ "Ext2 $(\mathrm{mm}) "$

Cells $(1,5)$.Value $=" \operatorname{Ext} 3(\mathrm{~mm}) "$

Cells $(1,6) \cdot$ Value $=$ "Ram LVDT

Cells $(1,7)$.Value $=$ "Segment"

Cells $(1,9)$.Value $=$ "Time Diff."

Cells $(1,10)$. Value $=$ "Stress"

Cells $(1,11)$.Value $=$ "Strain $($ Ext2)"

Cells $(1,12)$. Value $=$ "Strain $($ Ext3)"

Cells $(1,13)$.Value $=$ "Average Strain $($ Ext2 \& Ext3)"

Range("A1:M1").Select

Selection. Font. Bold $=$ True

Columns("B:B"). ColumnWidth $=8.57$

Columns("C:C").ColumnWidth $=9.14$

Columns("D:D"). ColumnWidth $=9$

Columns("F:F").ColumnWidth $=10.14$

Columns("H:H"). ColumnWidth $=1$

Columns("I:I"). ColumnWidth $=9$

Columns("K:K").ColumnWidth $=11$

Columns("L:L").ColumnWidth $=11$

Columns("M:M").ColumnWidth $=11$

Range("O20").Select

End Sub

Sub ComputePhaseLag(ipoints As Integer)

Dim RangeOfMinAndMax As MaxAndMinRange

Dim fMaxStress As Single, fMinStress As Single,

fStressPeak As Single

Dim iRowOfMaxStress As Integer, sRowOfMaxStress As

String

Dim fTimeForMaxStress As Single

Dim fMaxStrainDueToAverageStrain As Single,

fMinStrainDueToAverageStrain As

Single

Dim iRowOfMaxStrainDueToAverageStrain As Integer,

sRowOfMaxStrainDueToAverageStrain As String

Dim fTimeForMaxStrainDueToAverageStrain As Single

Dim fMaxStrainDueToExt2 As Single,

fMinStrainDueToExt2 As Single

Dim iRowOfMaxStrainDueToExt2 As Integer,

sRowOfMaxStrainDueToExt2 As

String
Dim fTimeForMaxStrainDueToExt2 As Single

Dim fStrainPeakDueToExt2 As Single

Dim fTimeOfStressAtEndOfCycle As Single,

fTimeOfStressAtStartOfCycle As Single

Dim fTimeLagDueToExt2 As Single,

fTimeLagDueToAverageStrain As Single,

fStrainPeakDueToAverageStrain As Single

Dim iStartRow As Integer, iEndRow As Integer, i As

Integer

Dim sShadedRange As String

iStartRow $=2$

iEndRow $=101$

For $\mathrm{i}=1$ To iNumberOfBlocks

'find maximum and min stress

RangeOfMinAndMax $=$ FindMaxValue(10, ipoints

iStartRow, iEndRow)

fMaxStress $=$ RangeOfMinAndMax.fMaxValue

$\mathrm{fMinStress}=$ RangeOfMinAndMax.fMinValue iRowOfMaxStress =

RangeOfMinAndMax.iRowNumberOfMaxValue sRowOfMaxStress = "A" \&

LTrim(Str\$(iRowOfMaxStress)) fTimeForMaxStress = Range(sRowOfMaxStress) . Value find maximum and min average strain RangeOfMinAndMax = FindMaxValue(13, ipoints,

iStartRow, iEndRow) fMaxStrainDueToAverageStrain $=$

RangeOfMinAndMax.fMaxValue fMinStrainDueToAverageStrain =

RangeOfMinAndMax.fMin Value

iRowOfMaxStrainDueToAverageStrain = angeOfMinAndMax.iRowNumberOfMaxValue sRowOfMaxStrainDueToAverageStrain = "A" \& LTrim(Str\$(iRowOfMaxStrainDueToAverageStrain)) fTimeForMaxStrainDueToAverageStrain = Range(sRowOfMaxStrainDueToAverageStrain).Value 'find strain based on Ext2 RangeOfMinAndMax = FindMaxValue(11, ipoints,

iStartRow, iEndRow) fMaxStrainDucToExt2 =

RangeOfMinAndMax.fMaxValue fMinStrainDueToExt2 =

RangeOfMinAndMax.fMinValue iRowOfMaxStrainDueToExt2 =

RangeOfMinAndMax.iRowNumberOfMaxValue sRowOfMaxStrainDueToExt2 = "A" \&

LTrim(Str\$(iRowOfMaxStrainDueToExt2)) fTimeForMaxStrainDueToExt2 =

Range(sRowOfMaxStrainDueToExt2).Value 'compute stress and average strains lag 'compute stress at 100 points, i.e at the end of the cycle fTimeOfStress AtEndOfCycle $=$ Cells $($ iEndRow, 1$)$. Value fTimeOfStressAtStartOfCycle $=$ Cells $($ iStartRow,

1).Value fTimeLagDueToExt2 = Abs $(($ fTimeForMaxStress -

fTimeForMaxStrainDueToExt2) $/$ (fTimeOfStressAtEndOfCycle -

fTimeOfStress AtStartOf(ycle)) * 360

fStressPeak = Abs(fMaxStress $-\mathrm{fMinStress)} * 0.5$ fStrainPeakDueToExt2 = Abs(fMaxStrainDueToExt2 .

fMinStrainDueToExt2) * 0.5 fTimeLagDueToAverageStrain $=$

Abs((fTimeForMaxStress fTimeForMaxStrainDueToAverageStrain) /

(fTimeOfStress AtEndOfCycle -

fTimeOfStressAtStartOfCycle)) * 360

fStressPeak $=$ Abs (fMaxStress $-\mathrm{fMinStress}) * 0.5$

fStrainPeakDueToAverageStrain $=$

Abs(fMaxStrainDueToAverageStrain fMinStrainDueToAverageStrain) * 0.5 
'show results

Cells(iStartRow, 14). Value = "Time Lag Due to Ext2 = " Cells(iStartRow, 15). Value $=$ fTimeLagDueToExt2

Cells(iStartRow $+1,14)$.Value $=$ "Time Lag Due to

Average Strain $=$ "

Cells $($ iStartRow $+1,15)$. Value $=$

fTimeLagDueToAverageStrain

Cells(iStartRow $+2,14)$. Value $=$ "Amplitude of Stress $=$

Cells $($ iStartRow $+2,15)$. Value $=$ fStressPeak

Cells(iStartRow $+3,14)$. Value $=$ "Amplitude of Strain

Due to Ext2 = "

Cells(iStartRow $+3,15)$. Value $=$ fStrainPeakDueToExt 2

Cells(iStartRow $+4,14)$.Value $=$ "Amplitude of Strain

Due to Average Strain = "

Cells(iStartRow $+4,15)$. Value $=$

fStrainPeakDueToAverageStrain

Columns("N:N"). ColumnWidth $=44$

Range("N2:N6").Select

With Selection

.HorizontalAlignment $=\mathrm{xlRight}$

VerticalAlignment $=$ xlBottom

WrapText $=$ False

. Orientation $=0$

. AddIndent $=$ False

ShrinkToFit $=$ False

. MergeCells $=$ False

End With

With Selection.Interior

.ColorIndex $=40$

Pattern $=$ xlSolid

End With

sShadedRange $=$ "N" \& LTrim(Str\$(iStartRow)) \& ":" \&

"O" \&

LTrim(StrS(iStartRow + 4))

Range(sShadedRange).Select

Selection.Interior.ColorIndex $=40$

Range("N10").Select

iStartRow $=$ iStartRow + iNumberOfPointsPerBlock +1

$\mathrm{i}$ EndRow $=\mathrm{iEndRow}+\mathrm{iNumberOfPointsPerBlock}+1$

Next

Range("A1:O1").Select

Selection.Interior. ColorIndex $=11$

Selection. Font.ColorIndex $=2$

End Sub

Function FindMaxValue(iColumnNumber As Integer, ipoints As Integer,

iStartRow As Integer, iEndRow As Integer) As

MaxAndMinRange

Dim Rng As Range

Dim fMaxNumber As Single, fMinNumber As Single

Dim RangeOfMinAndMax As MaxAndMinRange

Dim iRowOfMaxValue As Integer, i As Integer

'find max value

fMaxNumber = Cells(iStartRow, iColumnNumber). Value fMinNumber $=$ Cells (iStartRow, iColumnNumber). Value For $\mathrm{i}=\mathrm{iStartRow}+1$ To iEndRow +1

If Cells( $i$, iColumnNumber). Value $>$ fMaxNumber Then fMaxNumber $=$ Cells $(i$, iColumnNumber $)$.Value iRowOfMaxValue $=\mathrm{i}$

End If

If Cells(i, iColumnNumber).Value $<$ fMinNumber Then fMinNumber $=$ Cells $(i$, iColumnNumber $)$.Value

End If

Next

RangeOfMinAndMax.fMaxValue $=$ fMaxNumber RangeOfMinAndMax.iRowNumberOfMaxValue =

iRowOfMaxValue

RangeOfMinAndMax.fMinValue $=$ fMinNumber

FindMaxValue $=$ RangeOfMinAndMax
End Function

Sub ComputeStresses(sFileName As String, N As

Integer, fDiameter As Single,

fLength As Single)

Dim fArea As Single

Dim iRows As Integer

Dim fForce As Single, fStress As Single

Dim iRow As Long

Dim s_rangeOfRows As String

Dim i As Integer, j As Integer, iStartOfBlock As Integer

Dim iNumberOfPoints As Integer

Dim fEx2FirstReading As Single, fEx3FirstReading As Single

fArea $=3.1428571 *$ fDiameter $*$ fDiameter $* 0.25$

Cells $(65536,1)$.Select

Selection.End(xlUp).Select

For $\mathrm{i}=1$ To iNumberOfBlocks

iRow $=$ Selection. Row

s_rangeOfRows = "A" \& LTrim(Str\$(iRow))

s_rangeOfRows = s_rangeOfRows + ":A" \&

LTrim(Str\$(iRow - N + 1))

Range(s_rangeOfRows).Select

Range(Selection, Selection.End(xIToRight)).Select

Selection.Copy

Windows(sApplicationName).Activate

Sheets("sheet2").Select

Selection.Insert Shift:=xlDown

Application .CutCopyMode $=$ False

Range("A1").Select

Rows("1:1").Select

Selection.Insert Shift:=xlDown

Range("A1").Select

Windows(sFileName).Activate

Selection.End(xlUp).Select

Selection.End(xlUp).Select

Next

now compute stress in Sheet2

Windows(sApplicationName).Activate

Sheets("sheet2").Select

iNumberOfPoints $=$ iNumberOfBlocks *

iNumberOfPointsPerBlock

iStartOfBlock $=2$

For $\mathrm{i}=1$ To iNumberOfBlocks

Cells(iStartOfBlock, 1). Select

fEx2FirstReading = Cells(iStartOfBlock,

iExt2Column).Value

fEx3FirstReading $=$ Cells(iStartOfBlock,

iExt3Column).Value

For $\mathrm{j}=1$ To $\mathrm{iNumberOfPointsPerBlock}$

'stress

Cells(iStartOfBlock, 10). Value $=$ Cells $($ iStartOfBlock,

iForceColumn). Value /

fArea

'strain due to Ext2

Cells(iStartOfBlock, 11). Value $=$ Cells (iStartOfBlock,

iExt2Column).Value /

fLength

'strain due to Ext3

Cells $($ iStartOfBlock, 12) . Value $=$ Cells $($ iStartOfBlock,

iExt3Column).Value /

fLength

'strains average of Ext2 \& Ext3

Cells $($ iStartOfBlock, 13).Value $=($ Cells $($ iStartOfBlock,

11). Value +

Cells(iStartOfBlock, 12).Value) $* 0.5$

iStartOfBlock $=$ iStartOfBlock +1

Next

iStartOfBlock $=$ iStartOfBlock +1

Next

Application. CutCopyMode $=$ False 
End Sub

Sub DrawChartForEachBlock()

Dim sDestinationSheet As String, i As Integer

Dim iStartOfBlock As Integer, iSheetNo As Integer

Dim sChartTitle As String, sXaxisTitle As String,

sYaxisTitle As String,

sBlockRange As String, sChartLocation As String

Dim iChartTopLeft As Integer, iChartBottomRight As Integer

Dim sXValues As String, sYValues1 As String,

sYValues2 As String, .

sChartFirstSeriesRange As String

iSheetNo $=3$

iStartOfBlock $=2$

fFrequency $(1)=20$

fFrequency $(2)=10$

fFrequency $(3)=3$

fFrequency $(4)=1$

fFrequency $(5)=0.3$

fFrequency $(6)=0.1$

For $\mathrm{i}=1$ To iNumberOfBlocks

sDestinationSheet $=$ "Sheet" \& LTrim(Str\$(iSheetNo))

'draw stress

Sheets("Sheet2").Select

'select Time column

sBlockRange = "A" \& LTrim(Str\$(iStartOfBlock)) \&

":A" \&

LTrim(Str\$(iNumberOfPointsPerBlock +

iStartOfBlock))

'select Time block and stress block

sBlockRange = sBlockRange \& "," \& "J" \&

LTrim(Str\$(iStartOfBlock)) \& ":J" \&

LTrim(Str\$(iNumberOfPointsPerBlock +

iStartOfBlock))

Range(sBlockRange).Select

Charts. Add

sChartTitle $=$ "Stress vs. Time_For Frequency " \&

LTrim(Str\$(fFrequency(i)))

sYaxisTitle $=$ "Stress"

'position the chart

iChartTopLeft $=2$

iChartBottomRight $=20$

sChartLocation = "B" \& LTrim(Str\$(iChartTopLeft)) \&

":" \& "L" \&

LTrim(Str\$(iChartBottomRight))

Call DrawCharts(sBlockRange, sDestinationSheet,

sChartTitle, sYaxisTitle

sChartLocation)

'draw stain due to Ext2

Sheets("Sheet2").Select

select Time column

sBlockRange $=$ "A" \& LTrim $($ Str\$(iStartofBlock $)) \&$ ":A" \&

LTrim(Str\$(iNumberOfPointsPerBlock +

iStartOfBlock))

'select Time block and stress block

sBlockRange = sBlockRange \& ", \& "K" \&

LTrim(Str\$(iStartOfBlock)) \& ":K" \&

LTrim(Str\$(iNumberOfPointsPerBlock + iStartOfBlock))

Range(sBlockRange).Select

Charts.Add

sChartTitle $=$ "Strain vs. Time_Frequency " \&

LTrim(Str\$(fFrequency(i)))

sYaxisTitle = "Strain_Ext2"

'position the chart

iChartTopLeft $=$ iChartTopLeft +20

iChartBottomRight $=$ iChartBottomRight +20

sChartLocation = "B" \& LTrim(Str\$(iChartTopLeft)) \&

":" \& "L" \&
LTrim(Str\$(iChartBottomRight))

Call DrawCharts(sBlockRange, sDestinationSheet,

sChartTitle, sYaxisTitle,

sChartLocation)

'draw stain due to Ext3

Sheets("Sheet2").Select

'select Time column

sBlockRange $=$ "A" \& LTrim $($ Str\$(iStartOfBlock)) \&

":A" \&

LTrim(Str\$(iNumberOfPointsPerBlock +

iStartOfBlock)

' select Time block and stress block

sBlockRange = sBlockRange \& "," \& "L" \&

LTrim(Str\$(iStartOfBlock)) \& ":L" \&

LTrim(Str\$(iNumberOfPointsPerBlock +

iStartOfBlock))

Range(sBlockRange).Select

Charts.Add

sChartTitle = "Strain vs. Time_Frequency " \&

LTrim(Str\$(fFrequency(i)))

sYaxisTitle = "Strain_Ext3"

position the chart

iChartTopLeft $=$ iChartTopLeft +20

iChartBottomRight $=$ iChartBottomRight +20

sChartLocation = "B" \& LTrim(Str\$(iChartTopLeft)) \&

":" \& "L" \&

LTrim(Str\$(iChartBottomRight))

Call DrawCharts(sBlockRange, sDestinationSheet,

sChartTitle, sYaxisTitle,

sChartLocation)

'draw stress \& average strain due to Ext2 \& Ext3 vs. time

Sheets("Sheet2").Select

sChartTitle = "Stress \& Average Strain (Ext2 \& Ext3)

vs. Time_Frequency " \&

LTrim(Str\$(fFrequency(i)))

sYaxisTitle $=$ "Stress \& Average Strain vs. Time"

position the chart

iChartTopLeft $=$ iChartTopLeft +20

iChartBottomRight $=$ iChartBottomRight +20

sChartFirstSeriesRange $=" \mathrm{~A} "$ \&

LTrim(Str\$(iStartOfBlock)) \& ":A" \&

LTrim(Str\$(iNumberOfPointsPerBlock +

iStartOfBlock)

sChartFirstSeriesRange = sChartFirstSeriesRange \& "," \& "J" \&

LTrim(Str\$(iStartOfBlock)) \& ":J" \&

LTrim(Str\$(iNumberOfPointsPerBlock +

iStartOfBlock))

Range(sChartFirstSeriesRange).Select

Charts.Add

sChartLocation = "B" \& LTrim(Str\$(iChartTopLeft)) \&

":" \& "L" \&

LTrim(Str\$(iChartBottomRight))

sXValues = "=Sheet2" \& "!R" \&

LTrim(Str\$(iStartOfBlock)) \& "C1:" \& "R" \& LTrim(StrS(iNumberOfPointsPerBlock +

iStartOfBlock)) \& "C1"

sYValues2 = "=Sheet2" \& "!R" \&

LTrim(Str\$(iStartOfBlock)) \& "C13:" \& "R" \&

LTrim(StrS(iNumberOfPointsPerBlock +

iStartOfBlock)) \& "C13"

Call DrawStressStrainChart(sXValues, sYValues2,

sChartFirstSeriesRange, sDestinationSheet, sChartTitle, sYaxisTitle,

sChartLocation) iStartOfBlock $=$ iStartOfBlock $+1+$

iNumberOfPointsPerBlock

iSheetNo $=$ iSheetNo +1

Next 
Sheets("Sheet2").Select

End Sub

Sub DrawCharts(sBlockRange As String,

sDestinationSheet As String,

sChartTitle As String, sXaxisTitle As String,

sChartLocation As String)

ActiveChart.ChartType $=\mathrm{xlXYScatterSmooth}$

ActiveChart.SetSourceData

Source:=Sheets("sheet2").Range(sBlockRange),

PlotBy:=xlColumns

ActiveChart.Location Where:=xlLocationAsObject,

Name: $=$ sDestinationSheet

With ActiveChart

. HasTitle $=$ True

. ChartTitle. . Characters. Text $=$ sChartTitle

. Axes (xlCategory, xIPrimary) . Hastitle $=$ True

Axes(xlCategory, xlPrimary).AxisTitle.Characters.Text

$=$ "Time"

Axes(xlValue, $x l$ Primary) $\cdot$ Has Title $=$ True

.Axes(xlValue, $x I P$ Primary).AxisTitle. Characters. Text $=$ sXaxisTitle

With .Parent

Top = Range(sChartLocation) . Top

.Left $=$ Range(sChartLocation) . Left

.Width $=$ Range(sChartLocation). Width

Height $=$ Range(sChartLocation).Height

End With

End With

Call EditFonts

ActiveChart. HasLegend $=$ False

End Sub

Sub EditFonts()

ActiveChart.ChartTitle.Select

With Selection.Font

Name = "Arial"

FontStyle = "Bold"

Size $=10$

Strikethrough $=$ False

.Superscript $=$ False

Subscript $=$ False

OutlineFont $=$ False

.Shadow $=$ False

Underline $=x$ IUnderlineStyleNone

. ColorIndex $=$ xlAutomatic

Background = xlAutomatic

End With

ActiveChart.Axes(xlValue).AxisTitle.Select

With Selection.Font

Name = "Arial"

FontStyle = "Bold"

Size $=10$

Strikethrough $=$ False

Superscript $=$ False

Subscript $=$ False

.OutlineFont $=$ False

Shadow $=$ False

.Underline $=$ xlUnderlineStyleNone

ColorIndex $=$ xlAutomatic

. Background $=$ xlAutomatic

End With

ActiveChart.Axes(xlCategory).AxisTitle.Select
With Selection.Font

Name $=$ "Arial"

.FontStyle = "Bold"

Size $=10$

Strikethrough $=$ False

Superscript $=$ False

.Subscript $=$ False

OutlineFont $=$ False

Shadow $=$ False

Underline $=$ xIUnderlineStyleNone

. ColorIndex $=$ xlAutomatic

Background $=$ xlAutomatic

End With

End Sub

Sub DrawStressStrainChart(sXValues As String,

sYValues As String,

sChartFirstSeriesRange As String, sDestinationSheet As

String, sChartTitle As

String, sYaxisTitle As String, sChartLocation As String)

ActiveChart .ChartType $=$ xlXYScatterSmooth

ActiveChart.SetSourceData

Source:=Sheets("sheet2").Range(sChartFirstSeriesRange), _ PlotBy:=xlColumns

ActiveChart.Location Where:=xlLocationAsObject,

Name: $=$ SDestinationSheet

ActiveChart.SeriesCollection.NewSeries

ActiveChart SeriesCollection(2) $\cdot \mathrm{XV}$ alues $=\mathrm{sXV}$ Values

ActiveChart.SeriesCollection(2). Values $=\mathrm{s} Y$ Values

ActiveChart.SeriesCollection(2).Select

ActiveChart.SeriesCollection(2). AxisGroup $=2$

ActiveChart.SeriesCollection(1). Name = "="'stress vs

Time"'"!

ActiveChart.SeriesCollection(2).Name = "=""Average

Strain vs. Time""'"

With ActiveChart

. HasTitle $=$ True

. ChartTitle. . Characters. . Text $=$ sYaxisTitle

.Axes(xlCategory, xlPrimary). HasTitle $=$ True

Axes(xlCategory, xlPrimary).AxisTitle.Characters.Text $=s$ YaxisTitle

.Axes(xlValue, xlPrimary) HasTitle $=$ True

Axes(xlValue, xlPrimary).AxisTitle.Characters.Text = "Time"

With .Parent

Top $=$ Range(sChartLocation). Top

Left $=$ Range(sChartLocation) .Left

Width $=$ Range(sChartLocation). Width

.Height $=$ Range(sChartLocation $)$.Height

End With

End With

Call EditFonts

End Sub

Sub CloseAllWorkbookExceptActive()

Dim WB As Workbook

For Each WB In Workbooks

If WB.Name $<>$ ThisWorkbook. Name Then

WB.Close savechanges:=False

End If

Next WB

End Sub 


\section{REFERENCES}

1- Statistics Canada Agency, "Local Government Financial Management Statistics Capital Expenditures", Index 1733, 2003.

2- A. O. Abd El Halim; Y. Hassan and K. Kandil, "Factors Affecting Long-Term Performance of Pavement Overlay in Canada", 48th Annual Conference of the Canadian Technical Asphalt Association (CTAA), Halifax, Nova Scotia, November, 2003.

3- Transportation Association of Canada (TAC), Canadian Strategic Highway Research Program, "Canadian Long-Term Pavement Performance (C-LTPP) Program", Ottawa, Ontario, 1990.

4- "The State of Canada's Infrastructure", Executive Summary submitted to the Government of Canada, May 2000.

5- Transportation Association of Canada (TAC), Canadian Strategic Highway Research Program (C-SHRP), "Pavement Structural Design Practices Across Canada", CSHRP Technical Brief NO. 23, Ottawa, Ontario, April 2002.

6- Bruce Chadbourn, Shongatao Dai, P. Davich, J. Seikmeier, and D. VanDeusen, "Pavement Designer's Guide - Mn/DOT flexible Pavement design", MnPave Beta version 5.1, March 2002.

7- M. W, Witczak and E.J. Yoder, "Principles of Pavement Design", 2nd Edition, J. Wiley and Sons, N. Y. NY, 1975.

8- Hajeck, J. J., "General axle load Equivalent", Transportation Research Board, TRB, TRR 1482, NRC (U.S), Washington D.C., PP. 67-78, 1995.

9- Robert Lane, Graham Woodman and Ernest J. Barenberg "Pavement Design Considerations for Heavy Aircraft Loading At BAA Airport", Airport Pavement Innovations - Theory to Practice, Specialty Conference, American Society of Civil Engineers, 1993, pp. $90-109$.

10- Julie E. Kliewer and Ted S. Vinson, "Aging and Low-Temperature Cracking of Asphalt Concrete Mixture", Journal of Cold regions Engineering, Vol. 10, NO. 3, September 1996, pp. $134-148$.

11- A. C. Collop, D. Cebon, and M. S. A. Hardy, "Viscoelastic Approach to Rutting in Flexible Pavements", Journal of Transportation Engineering, Vol. 121, No. 1, January/February, 1995.

12- Namunu j., Meggod and Kuo Chang, "A Novel Approach to Develop a PerformanceBased Test for Rutting of Asphalt Concrete", Airport Pavement Innovations - Theory to Practice, Specialty Conference, American Society of Civil Engineers, 1993, pp. $126-140$.

13- Department of Transportation, U.S, "AASHO Interim Guide for the Design of Rigid and Flexible Pavements" published by the American Association of State Highway officials, Washington, D.C. 1961 
14- Department of Transportation, U.S, "AASHTO - 1972 Interim Guide for the Design of Pavement Structures", published by the American Association of State Highway and Transportation officials, Washington, D.C. 1972

15- Department of Transportation, U.S, "AASHTO Guide for the Design of Pavement Structures" published by the American Association of State Highway and Transportation officials, Washington, D.C. 1983

16-Van der Poel, "Time and Temperature Effects on the Deformation of Asphaltic Bitumens and Bitumen-Mineral Mixtures", SPE Journal, September 1955, pp. 47-53.

17-Department of Transportation, U.S, "AASHTO Guide for Design of Pavement Structures - 1993", published by the American Association of State Highway and Transportation officials, Washington, D.C. 1993.

18- The Asphalt Institute, "Mix Design Methods for Asphalt Concrete and Other Hot-Mix Types", Manual Series No. 2 (MS-2), May 1984.

19- National Crushed Stone Association, "Flexible Pavement Design Guide for highways”, NCSA Publication, Washington D.C., 1972.

20-US Corps of Engineers, "Developing a Set of CBR Design Curves", Instruction Report No. 4 Vicksburg, Mississippi, 1959.

21-AASHTO, "Standard Specifications for SuperPave Volumetric Mix Design", Designation MP2-02.

22- The Asphalt Institute, "Mix Design Methods for Asphalt Concrete and Other Hot-Mix Types", Manual series NO. 2 (MS -2) 4th Edition, March 1974.

23-American Association State Highway and Transportation Officials (AASHTO), "Standard Method of Test for Bulk specific gravity of Compacted Bituminous Mixtures Using Saturated Surface-Dry Specimens", Designation: T166 - 83.

24- American Association State Highway and Transportation Officials (AASHTO), "Standard Method of Test for Percent Air Voids in Compacted Dense and open Bituminous Paving Mixtures", AASHTO Designation: T 228 - 97

25-American Association State Highway and Transportation Officials (AASHTO), "Standard Test Method for Resistance to Plastic Flow of Bituminous Mixtures Using Marshall apparatus", AASHTO Designation: T 245 - 97 (2001).

26- Carl L. Monismith, "Analytically Based Asphalt Pavement design and rehabilitation: Theory to Practice, 1962-1992" Distinguished Lecture, Part 1, TRB 1992. Transportation Research Record 1354, Washington D.C. USA, pp. 72-80. P.S.

27- Kandhal, L.A. Cooley Jr., and Zhang, J. "Comparison of Fundamental and Simulative Test Methods for Evaluating Permanent Deformation of Hot-Mix Asphalt", Transportation Research Board, Transportation Research Record 1798, 2002.

28-The national Corporation Highway Research Program (NCHRP), "Simple Performance Tester for SuperPave Mix Design", Project 9-29.

29- Asphalt Institute, "SuperPave Mix Design Manual", SuperPave Series No. 2 (SP2), 3rd Edition, 2001. 
30-Consuegra, A., Little, D. N., Von Quintus, H. and Burati, J. "Comparative Evaluation of Laboratory Compaction Devices Based on Their Ability to Produce Mixtures with Engineering Properties Similar to Those Produced in the Field", Transportation Research Record No. 1228, pp. 80-87, 1989.

31-O. A. Fonseca and M. W. Witczak, "A Prediction Methodology for the Dynamic Modulus of In Place Aged Asphalt Mixture", Journal of the Association of Asphalt Paving Technologist, Vol. 65, 1996, pp. 532-565.

32-Zaiuddin A. Khan, Hammad I. Al-Abdul Wahab, Ibrahim Asi, Rezqallah Ramadhan, "Comparative Study of Asphalt Concrete Labotatorey Compaction Methods to Simulate Field Compaction", Journal of Construction and Building Materials, 12 (1998) $373-384$.

33-Chehab, G. R., O'Quinn, E., Kim, Y. R., "Specimen Geometry Study for Direct tension test Based on Mechanical Tests and Air Void Variation in SGC-Compacted Asphalt Concrete Specimens", NRC (U. S), 79th TRB meeting, TRR 1723, 2002, pp. $125-132$.

34- Van Til, C. J., et al "Evaluation of AASHTO Interim Guides for Design of Pavement Structures” NCHRP 128, Washington, D.C, 1972.

35-Department of Transportation, U.S, "Empirical -Mechanistic Design Guide for Design and Rehabilitation of Pavement Structures - 2002", published by the American Association of State Highway and Transportation officials, Washington, D.C. 2002.

36-Bruce Chadbourn, Shongatao Dai, P. Davich, J. Seikmeier, and D. VanDeusen, "Pavement Designer's Guide - Mn/DOT flexible Pavement design", MnPave Beta version 5.1, March 2002.

37- J. V. Heck, J.m. Piau; J. C. Gramsammer; J. P. Kerzreho and H. Odeon, "ThermoVisco- Elastic Modelling of Pavements Behaviour and comparison with Experimental Data from LCPC Test Track", 5th International Conference on the Bearing capacity of Roads and Airfields.

38- H. Di Benedetto and C. De La Roche, "State of the Art on Stiffness Modulus and Fatigue of Bituminous Mixtures', RILEM Report No. 17, 1998.

39-Stephen F. Brown, "Achievements and Challenges in Asphalt Pavement Engineering", 8th International Conference on Asphalt Pavements, Seattle 1977.

40- Y. Richard Kim, Youngguk, Mark King, and Mostafa Momen, "Dynamic Modulus Testing of Asphalt concrete in Indirect tension Mode", Submitted for presentation at the 2004 TRB Annual Meeting, Washington D. C., November 2003.

41- Ferry, J.D., "Visco-elastic properties of polymers", 3rd ed. Wiley, N.Y., 1980.

42-Sayegh, G., "Viscoelastic properties of bituminous mixtures", proceedings of the 2nd International conference on structural design of asphalt pavement, pp. 743-755.

43- Hopman, P.C, "VEROAD: A viscoelastic multilayer program", 75th TRB meeting, Transportation Research Record 1539, Washington D.C. USA, 1996, pp. 72-80. 
44-W. Zhang, Drescher, A., and D.E. Newcomb, "Viscoelasticity Behavior of Asphalt Concrete in Diametral Compression", Journal of Transportation Engineering, November/December 1997, pp. 495-502.

45-H. Di Benedetto, C. De La Roche, H. Baaj, A. Pronk and R. Lundström. "Fatigue of Bituminous Mixtures: Different Approaches and RILEM Group Contribution", Proceedings of the 6th International RILEM Symposium on Performance Testing and Evaluation of Bituminous Materials, Zurich, Switzerland, 14-16 April 2003.

46-Papazian, H.S., "The Response of Linear Viscoelastic materials in The Frequency Domain With Emphasis on Asphalt Concrete", 1st International conference on structural design of asphalt pavements. 1962, pp. 454-463.

47-Witczak M.W., and R.E. Root, "Summary of Complex Modulus Laboratory Test Procedures and Results", American Society for Testing and Materials, ASTM Special Technical Publication, Vol. 561, 1974, pp. 67-94.

48-Khanal, P.P. and M.S. Mamlouk "Tensile Versus Compressive Moduli of Asphalt Concrete", Transportation Research Record 1492, 1995, pp. 144-150.

49-Bonnaure, F., Gest, G., Gravios, A., and P. Uge, "A New method of Predicting the Stiffness of Asphalt Paving Mixtures", proceedings of Association of Asphalt Paving Technologists, Vol. 46, 1977, pp. 64-100.

50-Franken, L., Partl, M., "Complex Modulus Testing of Asphalt Concrete: RILEM International Test Program”, Transportation Research Record 1545, 1996, pp. 133142.

51-Drescher, A., Newcomb, D.E., and W. Zhang, "Interpretation of Indirect Tension Test Based on Viscoelasticity”, Transportation Research Record 1590, 1997, pp. 54-52.

52-NCHRP Project 9-19, Draft Test Protocol A1, "Dynamic Modulus of Asphalt Concrete Mixture and Master Curves".

53- NCHRP Project 9-29 "Simple Performance Tester for SuperPave Mix Design".

54- American Society for Testing and Materials (ASTM), "Standard Test Method for Dynamic Modulus of Asphalt Mixtures", ASTM D3497-79 (1995).

55-K. G. Sharp and A. Alderson, "Standard Method for the Laboratory Determination of the Elastic Modulus of Asphalt", Research Report No. AAR 210, Submitted to the Australian Road Research Board, Vermont South, Australia, July 1991.

56- Y. Richard Kim, Youngguk, Mark King, and Mostafa Momen, "Dynamic Modulus Testing of Asphalt concrete in Indirect tension Mode", Submitted for presentation at the 2004 TRB Annual Meeting, Washington D. C., November 2003.

57- Y. Z. Kim and Y. C. Lee, "Interrelationship among Stiffness of Asphalt - Aggregates Mixtures", Journal of the Association of Asphalt Paving Technologist, Volume 64, 1971, pp. 575-609.

58- Williams, M.L., Landel, F. R., and Ferry, J. D., "The Temperature Dependence of Relaxation Mechanism in Amorphous Polymers and Other Glass-Liquid", Journal of American Chemists Society, Vol. 77, 1955, pp. 370. 
59-Timothy R. C, Xinjun Li, Miahi O. M. and Eugene L. S.,"Dynamic and Resilient Modulus of MN/DOT Asphalt Mixture", Department of Civil Engineering, University of Minnesota, Report No. MN/RC - 2003-09, submitted to Minnesota Department of Transportation (MnDOT), February 2003.

60-NCHRP, "Modulus of Elasticity for Major Group Materials SuperPave Support and Performance Models Management”, 2002 Design Guide Draft - 2.4, Project 1-37A.

61-Pellin, T. K., and Witczak, M.W., "use of Stiffness of Hot-Mix Asphalt as a Simple Performance Test", Transportation Research Board (TRB) 2002 Annual Meeting, Washington D.C. USA,

62-Huhtala, M., "The Rheology of Bituminous Mixtures", EUROBITUME Workshop The Rheology of Bituminous Binders, Bruxelle, 1995.

63- Cost, "Development of a New Bituminous Pavement Design Method - Final Report of the Action", European Commission Directorate General Transport, Luxembourg, 1999.

64- Nilsson, R.N., Hopman, P. C., Isacsson, "Influence of different rheological models on predicted pavement responses in flexible pavement", International Journal for Road Materials and Pavement Design, Vol 3, Issue No.2/2002, pp. 117-149.

65-Witczak, M.W., Kaloush K. E., and H. Von Quintus, "Pursuit of the Simple Performance Test for Asphalt Mixture Rutting", Association of Asphalt Paving Technologists (AAPT), 2002 Symposium, Colorado Springs, CO.

66-American Association State Highway and Transportation Officials (AASHTO), "Standard Method of Test for Theoretical Maximum specific gravity and Density of Bituminous Paving Mixtures", AASHTO Designation: T 209 -94.

67-American Society for Testing and Materials (ASTM), "Standard Test Method for Dynamic Modulus of Asphalt Mixtures”, ASTM D3497-79 (1995).

68-American Association State Highway and Transportation Officials (AASHTO), "Standard Method of Test for Bulk specific gravity of Compacted Bituminous Mixtures Using Saturated Surface-Dry Specimens”, Designation: T166 - 83.

69-American Association State Highway and Transportation Officials (AASHTO), "Standard Method of Test for Theoretical Maximum specific gravity and Density of Bituminous Paving Mixtures", AASHTO Designation: T 209 -94.

70-American Association State Highway and Transportation Officials (AASHTO), "Standard Method of Test for Percent Air Voids in Compacted Dense and open Bituminous Paving Mixtures", AASHTO Designation: T 269 - 97.

71-Ontario Ministry of Transportation (MTO), Ontario Provincial Standard Specification, OPSS 1149 -1152, Ottawa, Ontario, July 1990.

72-Elton R. Brown, and M. Shane Buchanan, "Applicability of SuperPave to the Design Airfield Asphalt Pavement", Airfield Pavement Conference, August 1997, Seattle, Washington D.C 
73-Tony Geradi, "The effect of runway roughness on aircraft operations", Airfield Pavement Conference, August 1997, Seattle, Washington D.C

74-Ohio Department of Transportation (OhDOT), "1997 Construction and Material Specification".

Saskatchewan Ministry of Transportation, Standard Specifications Manual, Section 4100, Canada, Regina, January 1998. 